\title{
The Development and Application of Random Matrix Theory in Adaptive Signal Processing in the Sample Deficient Regime
}

\author{
by \\ Milutin Pajovic
}

Submitted in partial fulfillment of the requirements for the degree of

Doctor of Philosophy

at the

\section{MASSACHUSETTS INSTITUTE OF TECHNOLOGY}

and the

\section{WOODS HOLE OCEANOGRAPHIC INSTITUTION}

September 2014

(C) 2014 Milutin Pajovic. All rights reserved.

The author hereby grants to MIT and WHOI permission to reproduce and distribute publicly paper and electronic copies of this thesis document in whole or in part.

Author

Department of Electrical Engineering and Computer Science and

Joint Program in Applied Ocean Science and Engineering August 25, 2014

Certified by

Dr. James C. Preisig Associate Scientist with Tenure, WHOI Thesis Supervisor

Accepted by

Prof. Leslie A. Kolodziejski Chair, MIT EECS Committee on Graduate Students Accepted by

Prof. Henrik Schmidt

Chair, Joint Committee for Applied Ocean Science and Engineering 


\title{
The Development and Application of Random Matrix Theory in Adaptive Signal Processing in the Sample Deficient Regime
}

by

\author{
Milutin Pajovic
}

Submitted to the Department of Electrical Engineering and Computer Science and Joint Program in Applied Ocean Science and Engineering on August 25, 2014, in partial fulfillment of the requirements for the degree of Doctor of Philosophy

\begin{abstract}
This thesis studies the problems associated with adaptive signal processing in the sample deficient regime using random matrix theory. The scenarios in which the sample deficient regime arises include, among others, the cases where the number of observations available in a period over which the channel can be approximated as timeinvariant is limited (wireless communications), the number of available observations is limited by the measurement process (medical applications), or the number of unknown coefficients is large compared to the number of observations (modern sonar and radar systems). Random matrix theory, which studies how different encodings of eigenvalues and eigenvectors of a random matrix behave, provides suitable tools for analyzing how the statistics estimated from a limited data set behave with respect to their ensemble counterparts.

The applications of adaptive signal processing considered in the thesis are (1) adaptive beamforming for spatial spectrum estimation, (2) tracking of time-varying channels and (3) equalization of time-varying communication channels. The thesis analyzes the performance of the considered adaptive processors when operating in the deficient sample support regime. In addition, it gains insights into behavior of different estimators based on the estimated second order statistics of the data originating from time-varying environment. Finally, it studies how to optimize the adaptive processors and algorithms so as to account for deficient sample support and improve the performance.

In particular, random matrix quantities needed for the analysis are characterized in the first part. In the second part, the thesis studies the problem of regularization in the form of diagonal loading for two conventionally used spatial power spectrum estimators based on adaptive beamforming, and shows the asymptotic properties of the estimators, studies how the optimal diagonal loading behaves and compares the estimators on the grounds of performance and sensitivity to optimal diagonal load-
\end{abstract}


ing. In the third part, the performance of the least squares based channel tracking algorithm is analyzed, and several practical insights are obtained. Finally, the performance of multi-channel decision feedback equalizers in time-varying channels is characterized, and insights concerning the optimal selection of the number of sensors, their separation and constituent filter lengths are presented.

Thesis Supervisor: Dr. James C. Preisig

Title: Associate Scientist with Tenure, WHOI 


\section{Acknowledgments}

There are not enough words to describe my gratitude to people who have significantly impacted this research and contributed to my intellectual growth.

First of all, I am deeply indebted to my research supervisor and mentor Dr. James Preisig for offering me this wonderful opportunity and being so much helpful on every step on this long journey. Jim, thank you for giving me lot of academic freedom and making sure at the same time that I do not get lost in the wealth of opportunities I was presented to. Thank you for teaching me to seek insights in every mathematical expression and repeatedly asking for intuition and physical interpretation behind every analytical result. Thank you for being so supportive outside of academia and research when life faced me with some other challenges.

My academic advisor, Prof. Arthur Baggeroer, has been significantly involved into this research since my taking the RQE exam and his serving on the examination committee. Prof. Baggeroer, thank you for numerous meetings, for providing broader context of the research ideas we were chasing, for making me aware of the regularization problem (Chapter 3). Thank you also for being my "bibliography search engine" for different ideas and problems, and for providing a detailed feedback on the thesis document draft.

I am grateful to my other two thesis readers, Prof. Gregory Wornell and Prof. Raj Rao Nadakuditi for serving on my thesis committee and for their involvement in the discussions during our committee meetings. Greg, thank you also for giving me an opportunity to TA your course on Inference and Information, for training me how to deliver a course, and for making that experience unforgettable.

Prof. Alan Oppenheim deserves my special recognition for giving me home at MIT, welcoming me into his Digital Signal Processing Group (DSPG), and all his kindness and wisdom. Al, thank you for the impact you made in shaping my views on research and teaching in a very unique way.

I would like to say big thank you to Atulya Yellepeddi, Ballard Blair, and Sefa Demirtas for numerous and long discussions about even seemingly hopeless ideas. I 
am grateful to past and present members of the very unique DSPG: Charlie Rohrs, Dan Dudgeon, Dennis Wei, Guolong Su, Joe McMichael, Martin McCormick, Pablo Nuevo, Petros Boufounos, Qing Zhuo, Sally Wolfe, Sefa Demirtas, Shay Maymon, Tarek Lahlou, Tom Baran, Xue Feng, Yuantao Gu, Zahi Karam. Thank you all for making our weekly brainstorming sessions joyful and productive.

I would like to express big thanks to my friends from and outside of Boston, and in particular: Milo, thank you for your visits and lot of fun we have had together. Velibor, thank you for the support and great time we had last Summer. Thank you Selda and Sefa, and Keren, Yarden and Shay, for being beside us and helping us immensely when we were adjusting to our new roles.

My parents Ruza and Radomir have been great source of encouragement, support and inspiration thousands of miles away. Mum and Dad, thank you for taking good care of me, for believing in me when I didn't, and for pushing me to go beyond the expectations. Special thanks to my brother Rako, his wife Milica, and their little ones Sofija and Andrija, for all their prayers and love.

Finally, my wife Jelena and son Petar have shown me how joyful and fulfilling life could be. Jelena, thank you for leaving your career in Montenegro and joining me on this journey. Thank you also for bringing warmth to our home, and for pushing me to complete the thesis in time. Petar, your greeting smiles after my long days have given me the energy to endure all the difficulties and challenges; I devote this thesis to you!

The thesis would not have been possible without the generous support from the agencies that funded this research. I am grateful to the Academic Programs Office at WHOI, the Office of Naval Research (ONR Grants N00014-05-10085, N00014-0710738, N00014-09-10540, N00014-10-10259 and N00014-11-10426), and the National Science Foundation (NSF Grant ECCS-1102156) for their support. 


\section{Contents}

1 Introduction $\quad 13$

1.1 Adaptation with Second Order Statistics . . . . . . . . . . . . 14

1.1.1 Objective Function based on Second Order Statistics . . . . . 14

1.1.2 Ensemble Correlation and Sample Correlation Matrix . . . . . 15

1.2 Deficient Sample Support _ . . . . . . . . . . . . . . . . 16

1.3 Applications of Adaptive Processing . . . . . . . . . . . . . . . 21

1.3.1 Adaptive Spatial Spectrum Estimation . . . . . . . . . . . 21

1.3.2 Time-Varying Channel Tracking . . . . . . . . . . . . . . . 22

1.3.3 Communications Channel Equalization . . . . . . . . . 23

1.3.4 Underwater Acoustic Environment . . . . . . . . . . . . . 24

1.4 Thesis Objectives . . . . . . . . . . . . . . . . . 26

1.5 Organization of the Thesis . . . . . . . . . . . . . . . . . 29

2 Random Matrix Theory Methods 33

2.1 Introduction . . . . . . . . . . . . . . . . . . . . 33

2.2 Limiting Eigenvalue Density Function . . . . . . . . . . . . 35

2.3 The Stieltjes Transform . . . . . . . . . . . . . . . . . 36

2.3.1 The Eigenvalue and Eigenvector Stieltjes Transform . . . . . 36

2.3.2 The Eigenvalue Stieltjes Transform for an important model . . 39

2.3.3 The Eigenvector Stieltjes Transform for an important model . 41

2.4 Moments of Random Matrices . . . . . . . . . . . . . . . . . 43

2.5 Sample Correlation Matrix Models _. . . . . . . . . . . . . 45

2.5.1 The Moments of the Exponentially Weighted SCM _ . . . 45 
2.5.2 The Moments and Inverse of the Rectangularly Windowed SCM 49

2.5.3 White Noise Process . . . . . . . . . . . . . . 53

2.6 Gaussian Method for Random Matrices . . . . . . . . . . . . . . . . 57

2.7 Interpretation of Random Matrix Theory Results . . . . . . . . . . . 59

3 Spatial Power Spectum Estimation 63

3.1 Introduction . . . . . . . . . . . . . . . . 63

3.2 Background ...................... 66

3.3 Preview and Assumptions . . . . . . . . . . . . . . . . 69

3.3.1 Alternative Expressions for Power Estimators . . . . . . . . . 69

3.3.2 Gaussian Snapshot Model . . . . . . . . . . . . . . . . . . 70

3.3.3 Assumptions. . . . . . . . . . . . . . . . 72

3.3.4 Definitions of True Power . . . . . . . . . . . . . . . 73

3.3.5 Simulation Scenarios . . . . . . . . . . . . 74

3.4 Preliminary Results on Behavior of Power Estimators . . . . . . . . . 74

3.4.1 Dependence of Squared Bias on Diagonal Loading . . . . . . . 75

3.4.2 Dependence of Variance on Diagonal Loading . . . . . . . . . 78

3.5 Asymptotic Behavior of Power Estimators . . . . . . . . . . . . . . 81

3.5.1 Asymptotic Analysis of Power Estimators . . . . . . . . . 82

3.5.2 Asymptotically Unbiased Spatial Power Estimator . . . . . . . 85

3.5.3 Approximate Expectation of Power Estimators . . . . . . . . 86

3.5.4 Numerical Validation of Derived Expressions . . . . . . . . . 87

3.6 Mean Square Error of Power Estimators . . . . . . . . . . . . . . . . 90

3.6.1 Major Steps in the Derivation of Estimation MSE . . . . . . . 93

3.6.2 Numerical Validation of Derived Expressions . . . . . . . . . . 101

3.7 Optimization of Mean Square Error . . . . . . . . . . . . . 104

3.7.1 Estimation MSE versus Squared Bias Optimization . . . . . . 106

3.7.2 Estimation MSE Loss for Power Estimator $\hat{P}_{b}$. . . . . . . . 108

3.7 .3 Numerical Validation . . . . . . . . . . . . . . . . . . . 110

3.8 Behavior of Optimal Diagonal Loading . . . . . . . . . . . . . . . . . 119 
3.8.1 Single Source in Uncorrelated Noise . . . . . . . . . . . . 122

3.8.2 Multiple Sources in Uncorrelated Noise . . . . . . . . . . . 124

3.8.3 Alternative Definition of True Power . . . . . . . . . . . 128

3.8.4 Numerical Validation . . . . . . . . . . . . . . . . . 128

3.9 Comparison between Power Estimators . . . . . . . . . . . . . . . 131

3.9.1 Comparison based on Estimation Performance . . . . . . . . 132

3.9.2 Comparison based on Sensitivity to Optimal Diagonal Loading 136

3.9.3 Numerical Validation . . . . . . . . . . . . . . . . . . 136

3.10 Conclusions . . . . . . . . . . . . . . . . . . . 137

4 Time-Varying Channel Tracking 141

4.1 Introduction . . . . . . . . . . . . . . . . . . 141

4.2 Background . . . . . . . . . . . . . . . . . . . 143

4.2 .1 Problem Formulation . . . . . . . . . . . . . . . . 143

4.2 .2 Relevant Results . . . . . . . . . . . . . . . . . . . . 145

4.2 .3 Assumptions and Remarks . . . . . . . . . . . . . . 146

4.3 Performance Analysis . . . . . . . . . . . . . . . . . . 147

4.3.1 Channel Estimation Error . . . . . . . . . . . . . . . . . 148

4.3.2 Signal Prediction Error . . . . . . . . . . . . . . 150

4.3.3 Theoretical Prediction of Unknown Quantities . . . . . . . . 151

4.4 Channel Tracking in Steady State . . . . . . . . . . . . . . 158

4.4 .1 Performance Analysis . . . . . . . . . . . . . . . . . . . 158

4.4.2 Numerical Validation and Discussion . . . . . . . . . . 159

4.5 Linear-Time Invariant Channel Identification . . . . . . . . . . . 162

4.5 .1 Performance Analysis . . . . . . . . . . . . . . . . . . . . . 162

4.5.2 Theoretical versus Numerical Results . . . . . . . . . 166

4.5.3 Performance Deterioration . . . . . . . . . . . . . . . 170

4.6 Sliding Window RLS Algorithm . . . . . . . . . . . . . . . . 174

4.6.1 Performance Analysis . . . . . . . . . . . . . . . . . . . . . 174

4.6.2 Theoretical versus Numerical Results . . . . . . . . . . . 175 
4.6.3 Optimal Window Length . . . . . . . . . . . . . . . . 177

4.7 Insights in Exponentially Weighted and Sliding Window LS Algorithms 181

4.7.1 Effective Number of Observations . . . . . . . . . . . . . . . . 181

4.7.2 Exponentially Weighted versus Sliding Window RLS . . . . . 185

4.7.3 Optimal Value of Forgetting Factor . . . . . . . . . . . . . . 186

4.8 Conclusions . . . . . . . . . . . . . . . . . . . . . . 189

5 Channel Equalization $\quad 191$

5.1 Introduction . . . . . . . . . . . . . . . . . 191

5.2 Background . . . . . . . . . . . . . . . . 194

5.2.1 MC-DFE: Structure . . . . . . . . . . . . . 194

5.2 .2 MC-DFE: Analytical Framework . . . . . . . . . . . . 195

5.2.3 MC-DFE: Optimization of Weights . . . . . . . . . . . 197

5.3 Performance Analysis of Equalization of Time-Varying Channels . . . 199

5.3.1 Theoretical Analysis of Signal Prediction MSE . . . . . . . . . 199

5.3 .2 Numerical Validation . . . . . . . . . . . . . . . 204

5.4 Equalizer Design: Optimal Number of Coefficients . . . . . . . . . . 206

5.4.1 Insights into the Expression for Signal Prediction MSE . . . . 206

5.4 .2 Numerical Illustration . . . . . . . . . . . . . . . 208

5.5 Sparse Wideband Channel . . . . . . . . . . . . . . . 212

5.5.1 Arrival Model . . . . . . . . . . . . . . 213

5.5.2 Evaluation of Correlation Matrix R . . . . . . . . . . . 216

5.5.3 Evaluation of Cross-Correlation Vector $\mathbf{r}$. . . . . . . . . 217

5.6 Equalizer Design: Optimal Array Selection . . . . . . . . . . . . . 219

5.6.1 Optimal Sensor Separation . . . . . . . . . . . . . 219

5.6.2 Optimal Array Aperture . . . . . . . . . . . . . 222

5.6.3 Experimental Evidence . . . . . . . . . . . . 225

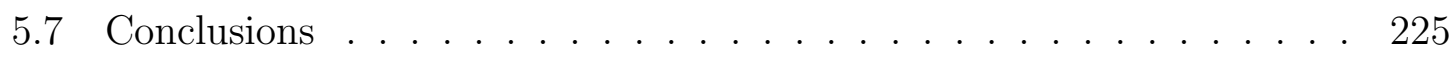

6 Conclusions and Future Work $\quad 229$

6.1 Random Matrix Theory . . . . . . . . . . . . . . 230 
6.2 Spatial Power Spectrum Estimation . . . . . . . . . . . . . . . . 231

6.3 Time-Varying Channel Tracking . . . . . . . . . . . . . . . . . . 232

6.4 Channel Equalization . . . . . . . . . . . . . . . . . . . 233

6.5 Future Work . . . . . . . . . . . . . . . . . 234

A Proof of Lemma 3.5 245

B Proof of Lemma $3.6 \quad 247$

C Optimization Loss $\quad 253$

$\begin{array}{lr}\text { Nomenclature } & 257\end{array}$ 


\section{Chapter 1}

\section{Introduction}

This thesis analyzes the performance of some types of adaptive signal processing algorithms when the number of observations available to adapt the characteristics of the algorithms is small. Adaptive processing algorithms, as considered in this thesis, are those whose goal is to track unknown parameters in real-time but which do not know a priori the statistics of those parameters or the observations. A general block diagram of an adaptive processor is shown in Fig. 1-1. The input data are processed such that the output is in some predefined way close to the reference (desired) signal. The coefficients, also called weights in a linear processor, are evaluated and updated based on the input signal, difference between the obtained and desired outputs and optimization criterion (i.e., objective or cost function) [27]. The format of the input, structure of the processor, objective function and unknown parameters depend on a specific application. Three applications of adaptive processing are considered in this thesis. These are the estimation of the spatial spectrum from observations at an array of sensors, the tracking of time-varying channels, and the equalization of communications channels. 


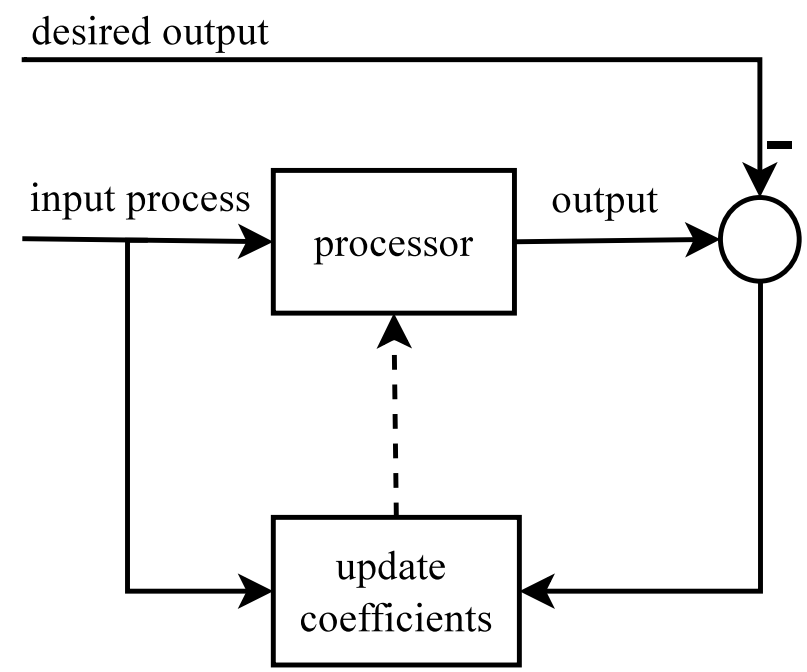

Figure 1-1: Block diagram of adaptive processor.

\subsection{Adaptation with Second Order Statistics}

\subsubsection{Objective Function based on Second Order Statistics}

The objective functions corresponding to the applications of adaptive processing considered in this thesis are such that the processor coefficients obtained as the solutions to the corresponding optimization problems depend on second order statistics of the input data. In other words, the unifying feature of the problems studied here is that the adaptive processing relies on the second order statistics of the data.

Although processing which utilizes higher order statistics is an option that has been extensively studied [34], this thesis focuses on processing with second order statistics for at least three reasons.

First, the second order statistics arise naturally in problems associated with Gaussian processes (completely characterized by their first and second order statistics) as well as problems utilizing Minimum Mean Square Error (MMSE) and Least Square (LS) error criteria with linear signal and processing models.

Second, the ensemble statistics of the data are unknown in practice and are estimated from the observed data. As will be argued shortly and studied more extensively in the thesis, the number of stationary observations is usually not sufficient to accurately estimate even the second order statistics. Estimating higher order statistics in 
this case becomes even more prohibitive.

Third, adaptive processing with higher order statistics requires more computations, which is a limiting factor in many practical applications. Greater computational capability requires, in general, a corresponding increase in power consumption which is often constrained in processors in underwater acoustic applications [10].

\subsubsection{Ensemble Correlation and Sample Correlation Matrix}

We emphasize that the objective functions corresponding to different adaptive processing applications relying on second order statistics are in general different. However, the solution for the processor weights in all applications depends on the second order statistics of the input data.

The input (also called received or observed) data at a particular time is a collection of measurements (i.e., samples) of the received signal. These measurements can be taken in spatial, delay, or both spatial and delay domains, and are arranged into an input (also called observation) vector $\mathbf{u}$. In general, $\mathbf{u} \in \mathbb{C}^{m \times 1}$, where $\mathbb{C}$ is the set of complex numbers and $m$ is the dimension of the observation space. ${ }^{1}$

The second order statistics of the input data are captured via correlations between measurements that constitute the observation vector $\mathbf{u}$. These correlations are formatted into an input correlation matrix, defined $\mathrm{as}^{2}$

$$
\mathbf{R}=\mathbf{E}\left[\mathbf{u u}^{H}\right]
$$

The expectation in the above definition is taken over the ensemble of observation vectors. Note that $\mathbf{R} \in \mathbb{C}^{m \times m}$.

The ensemble statistics of the input signal, and consequently the input correlation matrix $\mathbf{R}$, is usually unknown and has to be estimated from the observed data. Assuming the input process is ergodic, the ensemble statistics are estimated via time

\footnotetext{
${ }^{1}$ Note that the number of degrees of freedom is often smaller than the dimension of the observation space $m$. The focus of this thesis is not on developing and addressing the problems of lower dimensional representations.

${ }^{2}$ This is also the covariance matrix if the input process has zero mean. Without loss of generality, we assume throughout the thesis that all the input processes have zero mean.
} 
averaging. A widely used estimator for the input ensemble correlation matrix is the sample correlation matrix (SCM). The SCM is defined as

$$
\hat{\mathbf{R}}=\frac{1}{n} \sum_{k=1}^{n} \mathbf{u}(k) \mathbf{u}^{H}(k),
$$

where $\mathbf{u}(k)$ is the observation vector received at discrete time $k$, while $n$ is the length of the observation window.

It can be observed that the SCM is an unbiased estimator of the input correlation matrix. Also, the SCM is the maximum likelihood (ML) estimator of the ensemble correlation matrix when the snapshots are Gaussian distributed [52]. More importantly, for a fixed and finite number of coefficients $m$, as the number of observations $n \rightarrow \infty,[13]$

$$
\|\mathbf{R}-\hat{\mathbf{R}}\| \rightarrow 0, \quad \text { a.s. }
$$

where \|\| is a spectral norm of a matrix.

A practical interpretation of the above result is that the SCM is an accurate estimate of the input correlation matrix when the available number of observations $n$ used to compute the SCM is sufficiently large. The literature usually cites the empirical result that $n$ should be 3 times larger than $m$ when the input process has few dominant eigenvalues [43]. Some remarks on this result are made towards the end of the following section.

\section{$1.2 \quad$ Deficient Sample Support}

As pointed out in the previous section, if the number of observations $n$ is sufficiently many times larger than the number of coefficients $m$, the SCM is an accurate estimate of the input correlation matrix. However, this is rarely the case in the applications considered in this thesis and especially when operating in the underwater acoustic environment.

The problem of insufficient number of observations might arise as a result of one

or more of the following reasons. First, the statistics of the input signal might be 
non-stationary because the signal has propagated through a time-varying environment. A typical example is the wireless communication channel. Effectively, this means that the time interval over which the input signal can be assumed stationary is finite and possibly short [49]. Since the adaptation is performed using the statistics estimated from stationary observations, the number of observation vectors might not be sufficient to accurately estimate the correlation matrix.

Second, the length of the observation interval that can be used to estimate the time-varying statistics might not be sufficient. This typically arises in medical applications where only a limited number of measurements are taken in a diagnostic test.

Finally, the number of dimensions might be very large such that the number of observation vectors is small compared to the number of dimensions. The examples include a sonar system which nowadays might have hundreds to thousands of sensors or a sensor array in a modern seismic imaging system which might contain several thousands of sensors [46]. The number of observation vectors in such a scenario is often smaller than the number of sensors.

An important parameter we often refer to in the thesis is the ratio between the number of dimensions $m$ and observation vectors $n$,

$$
c=\frac{m}{n}
$$

Note that $1 / c$ is the average number of observations per matrix dimension.

We say that an adaptive processor operates in a deficient sample support (also called observation deficient) regime when the number of observations $n$ is smaller or not many times larger than the number of dimensions $m$. In terms of parameter $c$, the observation deficient regime arises when $c>1$ or $c$ is not much smaller than 1 . Although not formal, the notion of deficient sample support is important and helps in gauging the discussion in this thesis.

So far, we have pointed out that deficient sample support arises quite often in practice, especially in the applications studied in this thesis. Here, we qualitatively 
study how deficient sample support impacts the estimation accuracy of the SCM. In doing so, the eigenvalues of a matrix are chosen to conveniently visualize and intuitively infer if and how much the SCM departs from the corresponding ensemble correlation matrix. Two examples are considered as an illustration.

In the first example, we assume that $n$ observation vectors of a zero mean, unit power white noise process are received on $m$ sensors. The ensemble correlation matrix $\mathbf{R}$ is the identity matrix of order $m$ and therefore it has $m$ eigenvalues equal to one. To simulate how the eigenvalues of the SCM corresponding to white noise process behave, we perform the following numerical experiment [15]. A number of realizations of SCM's corresponding to white noise process are generated. Each SCM has order $m$ and is evaluated from $n$ different observation vectors of white noise process using (1.2). The eigenvalues of each SCM are computed, all obtained eigenvalues are collected and the normalized histogram, of area 1 , is evaluated.

The plots of normalized histograms for $m=10$ sensors and $n=20$ and $n=60$ observation vectors are respectively shown in the top and bottom part of Fig. 1-2. As can be observed, the eigenvalues of the SCM are spread around the ensemble (i.e, true) eigenvalue 1 (whose multiplicity is 10). This indicates that the SCM and ensemble correlation matrix differ. The amount of spread gives an intuitive indication of how much the SCM departs from the ensemble correlation matrix.

Finally, note that as the number of observations per dimension $1 / c$ increases from 2 (top figure) to 6 (bottom figure), the eigenvalues of the SCM concentrate around the ensemble eigenvalue.

In the second example, the input process is of zero mean and its correlation matrix is such that it has three distinct eigenvalues: 2, 5 and 7 . The process is measured on $m=3$ sensors and $n=15$ stationary observation vectors are available for computing the SCM. We perform the same numerical experiment as in previous example. Namely, a number of SCM's, each of order $m=3$ and computed from $n=15$ different observation vectors, are generated. The eigenvalues of each realization are computed and the normalized histogram of all eigenvalues is evaluated. The histogram is shown in Fig. 1-3. 

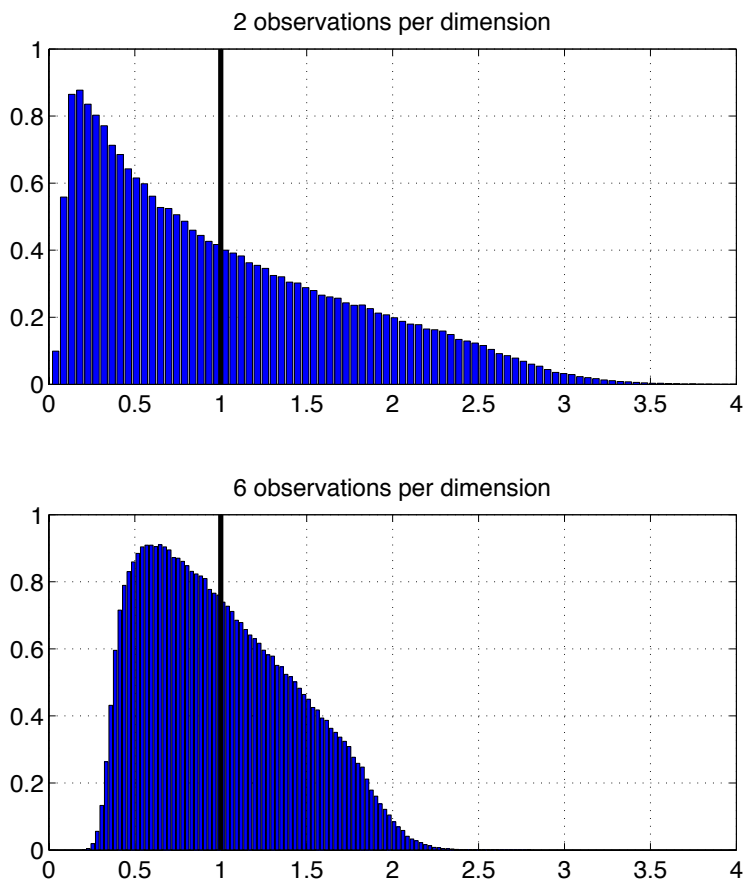

Figure 1-2: Normalized histograms of the eigenvalues of sample correlation matrices corresponding to zero mean, unit power, white noise process measured on $m=10$ sensors. The number of observations $n$ is 20 in the top plot and 60 in the bottom plot. The ensemble eigenvalue is 1 . 


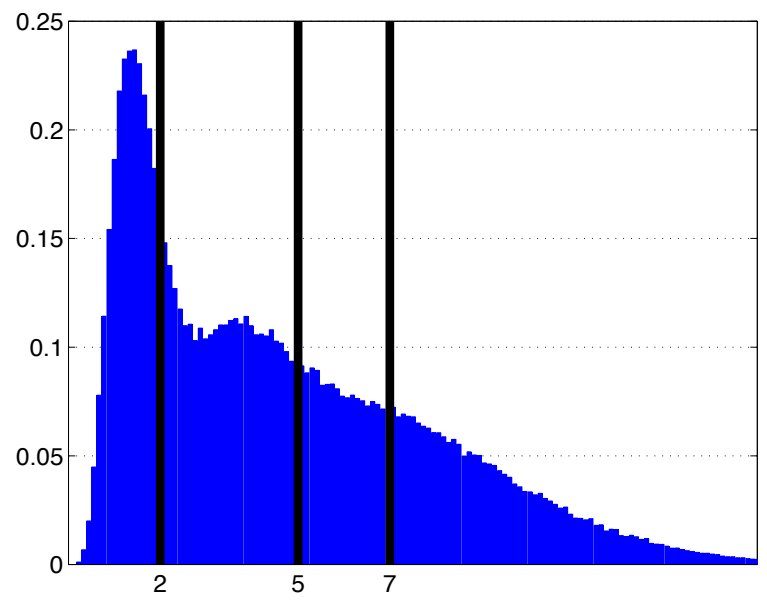

Figure 1-3: Normalized histogram of the eigenvalues of sample correlation matrices corresponding to zero mean, colored process whose ensemble eigenvalues are: 2, 5 and 7 . The process is measured on $m=3$ sensors and $n=15$ observation vectors are received.

The histogram plot in 1-3 shows that the eigenvalues of the SCM are spread around the ensemble (i.e., true) eigenvalues of the input process. More specifically, inferring the ensemble eigenvalues from the normalized histogram itself would be a daunting task. This indicates that the SCM and ensemble correlation matrix differ. More importantly, this happens even though the number of observation vectors is 5 times larger than the number of sensors (i.e., the number of dimensions) !

As a final remark, we point out that in addition to an empirical result from [43] stating that 3 observations per dimension are sufficient to accurately estimate the SCM, the separation between distinct ensemble eigenvalues also plays its role. Namely, as shown in the considered example, 3 observations per dimension are not sufficient in order to have the sample eigenvalues start falling in non-overlapping segments around their ensemble counterparts. However, this number might be sufficient if the ensemble eigenvalues were well separated. 


\subsection{Applications of Adaptive Processing}

This section briefly overviews the three applications of adaptive processing that are studied in the thesis. Their unifying feature is that a problem of deficient sample support often arises when those applications operate in practical settings. This is in particular the case in underwater acoustic environment, which is our primary interest.

The notion of deficient sample support is highlighted in the overview of each application. The illustration is provided in the last part with results obtained from processing the underwater acoustic data collected in a field experiment.

\subsubsection{Adaptive Spatial Spectrum Estimation}

Spatial power spectrum estimation consists of estimating the power received at an array of sensors as a function of direction of arrival. ${ }^{3}$ This is usually used in the context of estimating the number of point source signals embedded in the signal that is received at an array and then estimating their direction of arrival and power. Due to time-varying nature of the environment, these quantities are changing in time and an adaptive beamformer tracks the spatial spectrum in real time.

A block diagram of an adaptive beamformer is shown in Fig. 1-4. An array of sensors is spatially sampling the received signal. The Fourier transform of the signal received on each sensor is computed. The Fourier coefficients across the array corresponding to a particular frequency bin of interest is the observation vector for an adaptive processor. These observation vectors are often called snapshots.

The snapshots are processed through an adaptive processor and its output is an estimate of the spatial spectrum in a particular direction and frequency bin of interest. The adaptive processor is a linear, time-varying spatial filter which contains a single tap per each sensor. The coefficients of the processor are computed and adapted based on the estimated statistics of the received signal [52]. The number of coefficients being adapted is equal to the number of sensors and is denoted by $m$.

The number of stationary snapshots $n$, used to compute the SCM and in turn

\footnotetext{
${ }^{3}$ Note that the spatial spectrum can be mapped into the wavenumber spectrum.
} 


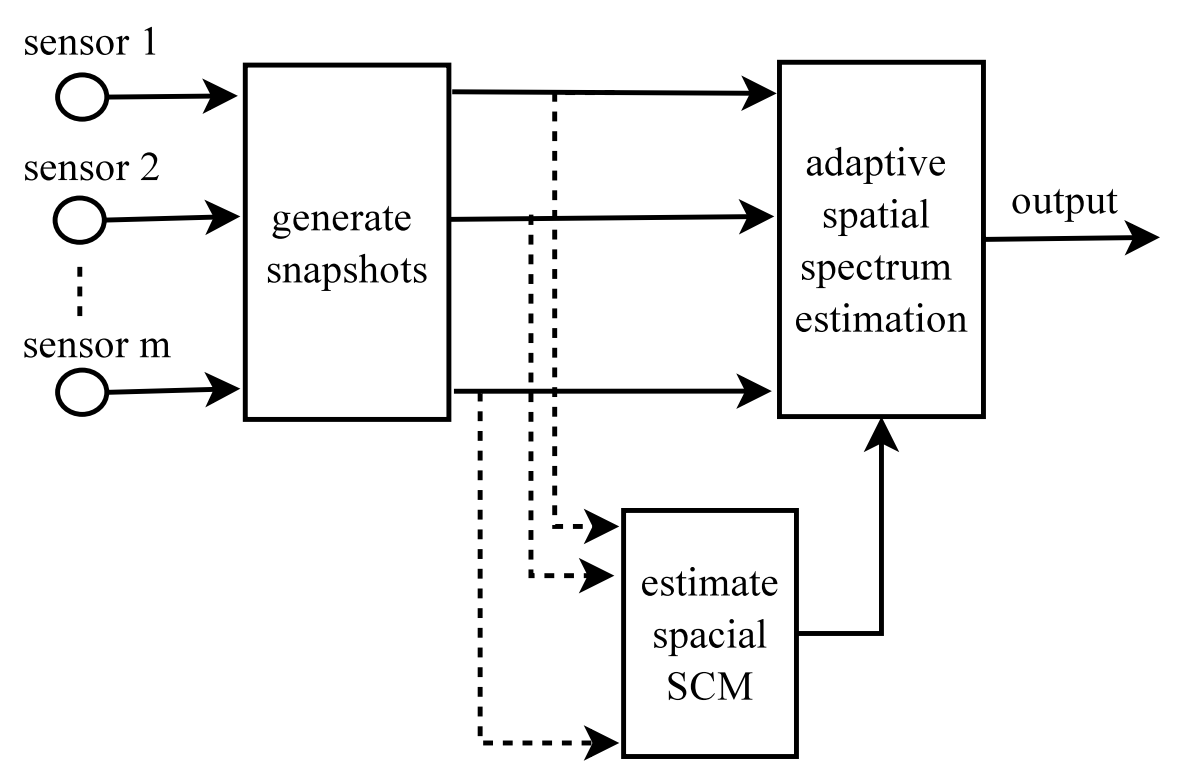

Figure 1-4: Adaptive spatial spectrum estimation.

the processor coefficients, is finite and often limited due to time-varying nature of the environment. Therefore, the adaptive processor often operates in the sample deficient regime.

\subsubsection{Time-Varying Channel Tracking}

A block diagram of a channel tracking problem framed as an adaptive processing problem is shown in Fig. 1-5. The unknown channel is modeled as a linear, timevarying finite impulse response (FIR) filter. Observations of the signal that has passed through the channel are contaminated with observation noise. The adaptive processor has access to channel inputs and noisy channel outputs. The estimated channel impulse response (also called channel vector) is updated at discrete time $t$ based on the estimated channel impulse response at time $t-1$ and channel input and observed noisy output at time $t[27][41]$.

Following the terminology introduced in Section 1.1, the observation vector at discrete time $t$ is formed from the input data samples which impact the channel output at time $t$. The number of these samples is equal to the number of unknown channel coefficients and is denoted with $m$. Note that this is the dimensionality of the observation space. 


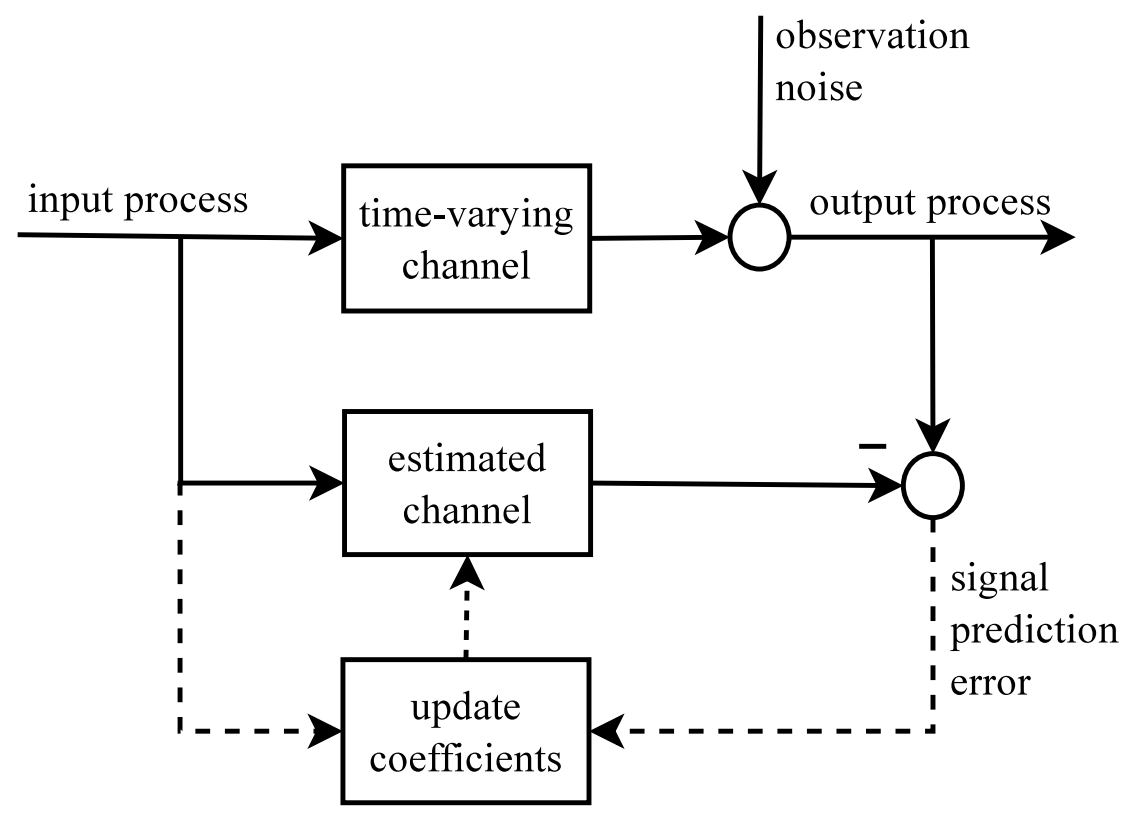

Figure 1-5: Channel tracking.

The number of observation vectors $n$, viewed in the context of previous section, is tied with how rapidly the channel varies in time. More specifically, even though the channel input might originate from a stationary process, the adaptive processor might still operate in the sample deficient regime. Namely, the channel impulse response at a particular time instant is estimated from the inputs and noisy outputs observed during the time interval over which the channel is approximately time-invariant such that the output data is approximately stationary.

\subsubsection{Communications Channel Equalization}

The wireless communications channels through which signals are often transmitted are often time-varying and characterized by multipath propagation, which then results in intersymbol interference and Doppler spreading of the signal. Most techniques developed for mitigating these effects rely in part or completely on channel equalization.

A block diagram of an equalizer is shown in Fig. 1-6. The equalizer input are samples of the received signal. The equalizer is a processor which coherently combines the received signal energy and consequently compensates for the intersymbol 
interference introduced in the channel. A decision device estimates the transmitted symbols from the equalizer outputs [41].

The equalizer coefficients depend on channel impulse response and are therefore adapted according to channel variations. The block diagram in Fig. 1-6 corresponds to an equalizer with direct adaptation. Depending on the position of the switch, an equalizer operates in either training or decision directed mode. The coefficients of an equalizer operating in the decision directed mode are, at discrete time $t$, updated based on the received signal and the difference between the detected symbol and its soft estimate at discrete time $t-1$. On the other hand, the transmitted symbols are known a priori when an equalizer operates in a training mode such that the coefficients are updated based on the received signal and the difference between the transmitted symbol and its soft estimate.

The number of equalizer coefficients $m$ is the number of dimensions. The estimate of input correlation matrix is essential in the computation and adaptation of equalizer coefficients when the objective function is based on second order statistics.

In the context of Section 1.1, the observation vector at discrete time $t$ is a vector of appropriately arranged input samples of the received signal which impact the detection of a transmitted symbol at time $t$. The number of stationary observation vectors $n$, used in the computation of the SCM, depends on how rapidly the transmission channel varies in time. The relative ratio between the number of equalizer coefficients $m$ and number of observation vectors $n$ might be such that the adaptation is performed with deficient sample support.

\subsubsection{Underwater Acoustic Environment}

The coefficients of adaptive processors described in the previous section are quite often adapted with deficient sample support. The causes might be time-varying channel, non-stationary environment, large number of sensors or their combinations. This is especially the case in the underwater acoustic setting where the underwater acoustic signals are adaptively processed. This in fact is our main motivation for studying adaptive processing with deficient sample support. The particular applications are 


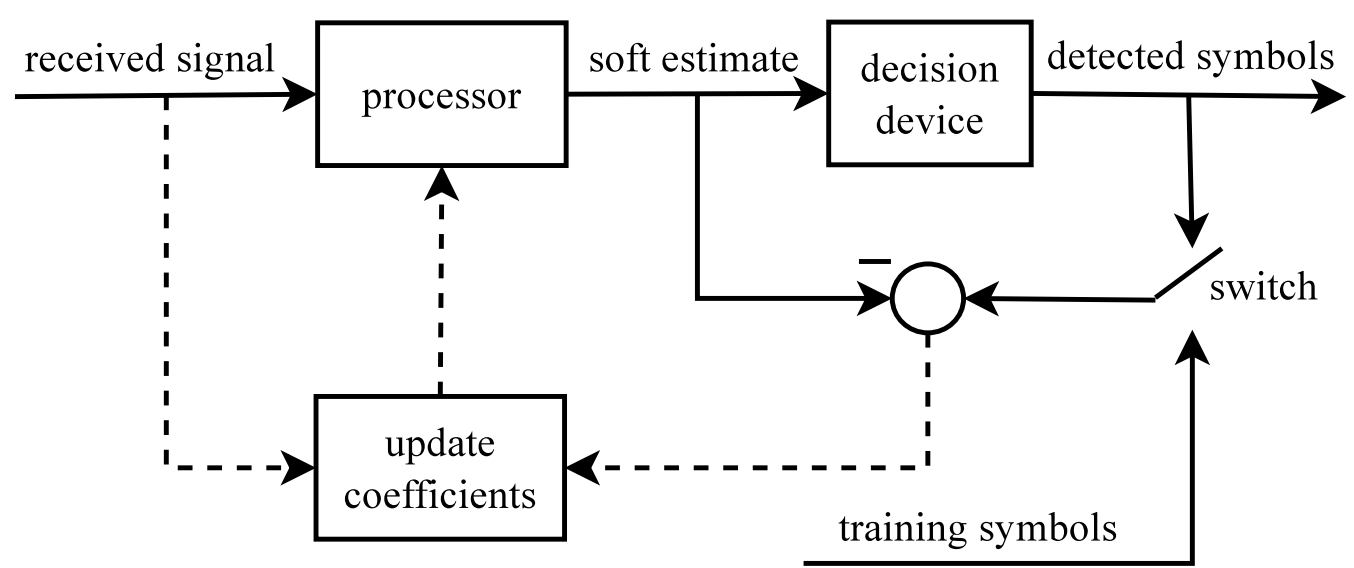

Figure 1-6: Channel equalizer with direct adaptation.

underwater acoustic communications and passive sonar.

The main challenges associated with adaptive processing of underwater acoustic signals arise from the time variability of the underwater acoustic environment [2], [51]. The time variability of the environment is caused by unavoidable drifts of the receiver, motions of the sources, surface and internal waves, and other moving objects such as fish. These result in finite and often short coherence intervals during which the observations are statistically stationary. The time variability of the environment is usually quantified by the scattering function [58].

In addition to causing deficient sample support, an underwater acoustic environment poses a number of other challenges on shallow and deep water underwater acoustic system design [51], [2]. As such, the motions in the underwater acoustic environment together with a relatively small speed of propagation (nominally $1500 \mathrm{~m} / \mathrm{s}$ ) result in relatively large Doppler spread of the received signals. Further, the acoustic waves exhibit multiple bounces off the surface and bottom in shallow waters, which results in long delay spread of the received signal. The delay spread of the communications signals may extend over several tens to hundreds of transmitted symbols. Also, the attenuation of the underwater sound is frequency dependent. Finally, the ambient noise is non-Gaussian and correlated in space and time.

To illustrate the time-variability of underwater acoustic environment and challenges associated with processing the underwater acoustic signals, some results obtained from processing the acoustic signals measured in a field experiment are pre- 
sented. The field experiment, labeled KAM11, took place in the shallow water off the coast of Hawaii in summer 2011 with one goal being to study the underwater acoustic communication channel [28].

The impulse response of time-varying underwater acoustic channel estimated from the data collected in the field experiment is shown in Fig. 1-7. The horizontal and vertical axes represent the time and delay, respectively. Therefore, a vertical slice in Fig. 1-7 is the channel impulse response at the corresponding time instant. As can be observed, the channel impulse response exhibits time-variations even on short time scales.

The acoustic signals recorded in the field experiment are received on a linear, vertical, uniformly spaced 24-sensor array. The distributions of the received acoustic energy over the space of elevation angles and delays at two different time instants are shown in Fig. 1-8. The elevation angle is defined with respect to the array such that $90^{\circ}$ corresponds to the broadside of the array and $0^{\circ}$ corresponds to signals traveling up from below. The time difference between the plots in the top and bottom part is 45 seconds. The arrival structure of the received signal fluctuates in time and while the number of arrivals and their directions do not vary significantly in the time interval of 45 seconds, the amount of energy associated with different arrivals changes in this time period.

\subsection{Thesis Objectives}

This thesis studies the problem of adaptive processing in the deficient sample support regime. The applications of adaptive processing considered are adaptive beamforming for spatial spectrum estimation, tracking of time-varying channels and equalization of communication channels. The computation and adaptation of coefficients in the considered adaptive processors are based on the estimates of second order statistics of the data. The unifying feature of the considered applications is that the number of observations is quite often insufficient to accurately estimate the second order statistics. This is especially the case in the underwater acoustic environment, which is 


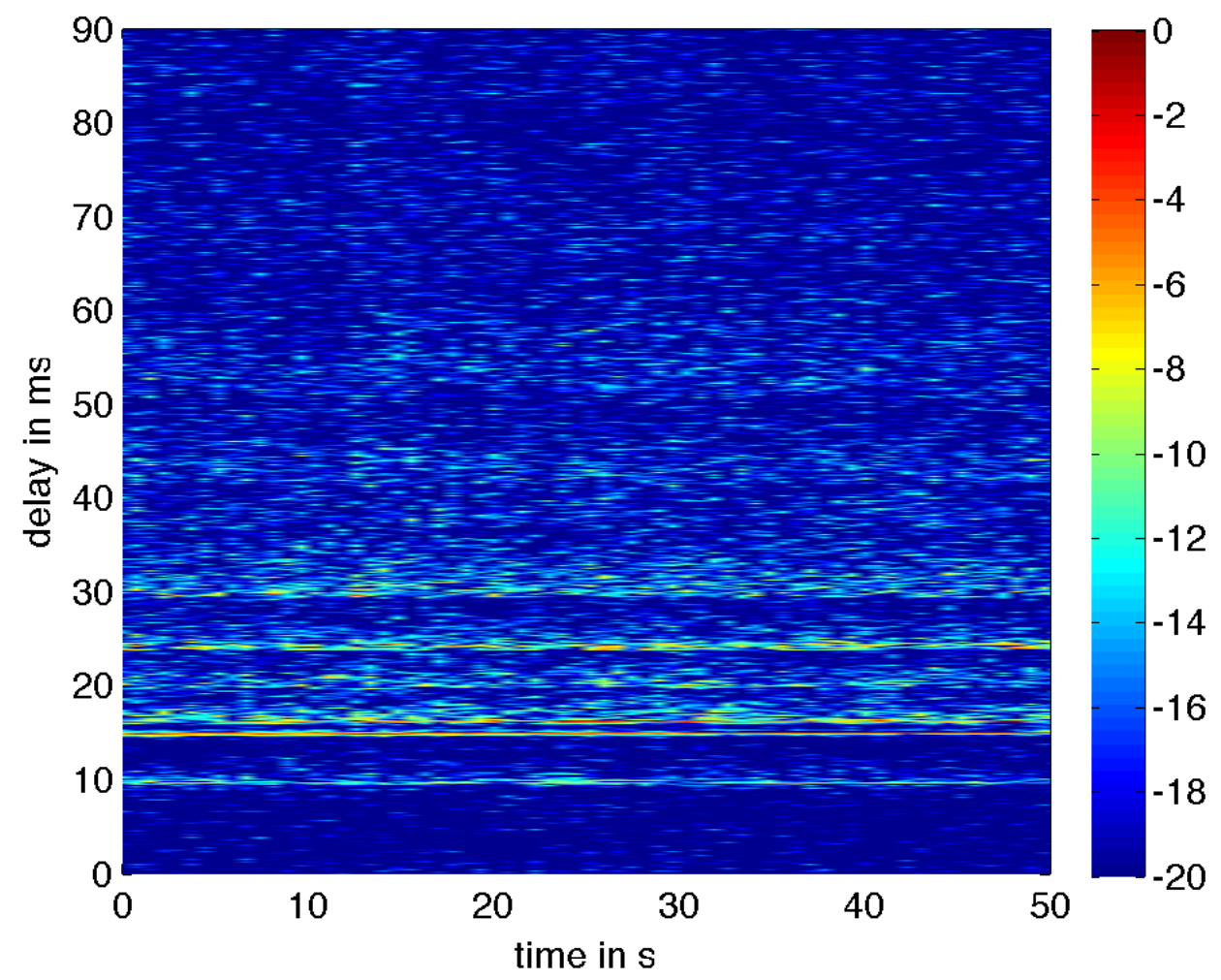

Figure 1-7: Impulse response of an underwater acoustic communication channel estimated from the KAM11 field data. The color scale is in dB. 

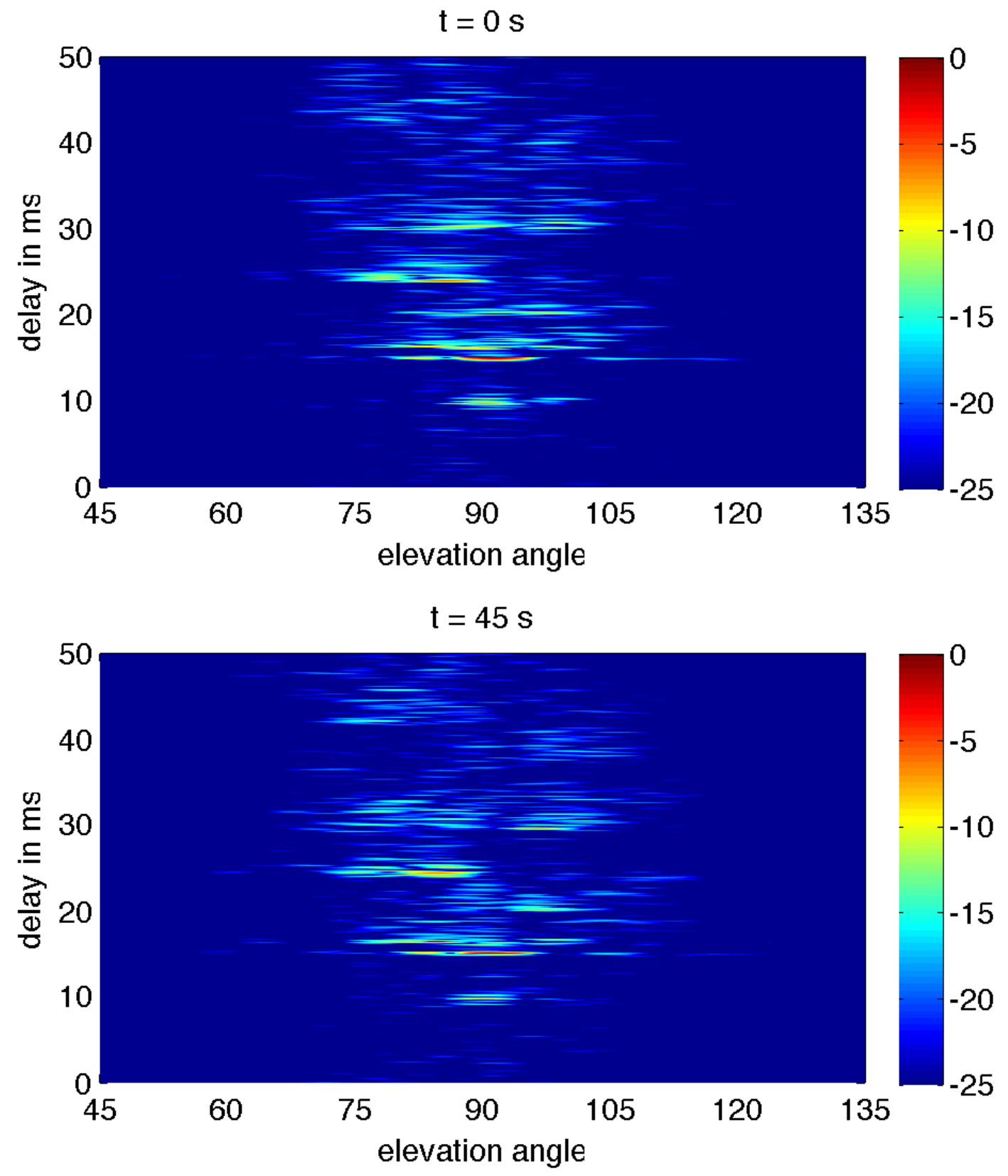

Figure 1-8: Distribution of received acoustic energy versus elevation angle and delay at the initial time instant (top plot) and after 45 seconds (bottom plot). The data used to generate these plots was collected in KAM11 field experiment. The color scale is in $\mathrm{dB}$. 
our main motivation. However, the issues associated with adaptation in the deficient sample support regime often arise in many other practical applications and settings.

The main tool used for studying the adaptation with deficient sample support is random matrix theory. The random matrix theory characterizes the eigenvalues and eigenvectors of different classes of random matrices. A sample correlation matrix, which estimates the correlation structure of the input process, is a random matrix of our interest. Consequently, the thesis can be viewed as an application of random matrix theory methods for addressing the problems of adaptive processing.

In short, the thesis analyzes the performance of the considered adaptive processors when operating in the deficient sample support regime. In addition, it gains insights into behavior of different estimators based on the estimated second order statistics of the data originating from time-varying environment. Finally, it studies how to optimize the adaptive processors and algorithms so as to account for deficient sample support and consequently improve the performance.

\subsection{Organization of the Thesis}

The thesis is organized as follows.

Chapter 2 presents background on random matrix theory methods and evaluates important quantities needed for the performance analysis in later chapters. More specifically, the eigenvalue density functions, eigenvalue and eigenvector Stieltjes transforms and moments of a random matrix are defined. The random matrix models which describe sample correlation matrices used in the thesis are presented and important theorems which characterize Stieltjes transforms corresponding to these models are stated. Using these characterizations, the moments of the considered sample correlation matrices are evaluated. In addition, two important results which characterize the expectation and variance of functionals of Gaussian matrices are stated. Finally, the chapter is concluded with the discussion on how the asymptotic random matrix theory results are used in practical, non-asymptotic, scenarios. 
Chapter 3 considers a problem of spatial power spectrum estimation with an array of sensors in the deficient sample support regime. More specifically, the problem of regularization ${ }^{4}$ in the form of diagonal loading of a sample correlation matrix, used in two spatial power spectrum estimators, is studied. In particular, the asymptotic behavior of two spatial power estimators, their expectations, variances and MSE's are analyzed in the limit when the number of snapshots and number of sensors grow large at the same rate. Due to rapid convergence, the limiting values accurately approximate the corresponding quantities for finite number of sensors and snapshots. Further, the study of dependence of the bias and variance corresponding to power estimators on diagonal loading leads to a conjecture that the variance has negligible impact on the value of optimal diagonal loading which minimizes the MSE. The behavior of optimal diagonal loading when the arrival process is composed of plane waves embedded into uncorrelated noise is investigated. Finally, the MSE and sensitivity performances of the optimized power estimators are compared.

Chapter 4 presents a performance study of the RLS algorithm when it is used to track a channel which varies according to a first order Markov process. The expressions for signal prediction and channel estimation mean square errors (MSE) are derived and validated via simulations. The general results are applied for specific scenarios and as special cases the behavior in the steady-state, performance of LSbased identification of linear time-invariant channel and performance of the sliding window RLS algorithm are considered. Finally, several practical results such as those characterizing the optimal exponential forgetting factor in the exponentially weighted RLS or optimal averaging window length in the sliding window RLS algorithm, are obtained.

Chapter 5 presents a performance study of the least squares based multi-channel Decision Feedback Equalizer when the transmission channel is non-stationary and modeled as a frequency selective filter which is time-invariant over only short time intervals. The expression for signal prediction MSE is derived and validated via MonteCarlo simulations. Further, it is elaborated that the optimal number of equalizer coef-

\footnotetext{
${ }^{4}$ Also called, Tikhonov regularization and relaxation.
} 
ficients is a trade off between two competing requirements such that an equalizer with relatively short constituent filters can outperform one using longer filters. Finally, the impact of the number of sensors and separation between them on the equalization performance of a time-varying underwater acoustic communication channel is studied. The insights concerning the optimal selection of the number of and separation between sensors as well as the lengths of the constituent filter are validated using the data collected in a field experiment.

Chapter 6 summaries the context and adaptive processing problems addressed in this thesis, highlights the contributions and suggests possible future work. 


\section{Chapter 2}

\section{Random Matrix Theory Methods}

\section{$2.1 \quad$ Introduction}

Random matrix theory is a mathematical area concerned with the characterization of random matrices. It is usually classified into small and large dimensional (asymptotic) random matrix theory.

The small dimensional theory studies random matrices of finite dimension. The small dimensional theory includes the very first random matrix theory result leading to the joint probability density function of the entries of the Wishart matrix, which is effectively the SCM of the Gaussian observation process [61]. A brief overview of the relevant results in small dimensional theory is given in [13]. A compact survey of the relevant results from the numerical analysis perspective, along with a broader perspective of the subject and its intimate connections with the orthogonal polynomials is given in [16].

The large dimensional theory studies how different transforms of eigenvalues and eigenvectors behave asymptotically when the order of an underlying random matrix grows. The birth of large dimensional theory is usually attributed to the Wigner semi-circle law [59], [60] and Marcenko-Pastur law [33]. Since then, the asymptotic behavior of random matrices has been extensively studied. Nice overviews of the results relevant for the applications is engineering areas are given in [54] and Part I of [13]. The large dimensional random matrix theory is of interest in this thesis and 
we simply refer to it as random matrix theory.

The random matrix theory has been successfully applied in information theory and wireless communications since the first result reported in [53]. An overview of random matrix theory applications for studying a variety of communication techniques from the information theory perspective is given in Part II of [13]. The random matrix theory has also been applied in detection and estimation such as for developing new algorithms for detecting and estimating the number of sources [42], [30].

This thesis exploits the random matrix theory insights and results in the study of adaptive processing problems associated with adaptation in the deficient sample support regime. More specifically, we study the problems of time-varying channel estimation, diagonal loading for spatial spectrum estimation with small number of snapshots and equalization of time-varying wideband communications channels. The random matrix theory is a convenient tool used in the analysis of these problems. Different performance metrics are asymptotically characterized and the obtained expressions are used to approximate the cases of practical interest.

This chapter introduces the fundamental concepts in random matrix theory, presents the results relevant for our analysis and evaluates important quantities whose characterization is necessary for the study of adaptive processing problems in the rest of the thesis.

The rest of the chapter is organized as follows. Section 2.2 defines fundamental quantities in random matrix theory. Section 2.3 defines eigenvalue and eigenvector Stieltjes transforms and presents two important theorems which characterize the asymptotic behavior of these transforms for the random matrix models of our interest. Section 2.4 defines limiting moments of a random matrix and elaborates how they can be computed from the Stieltjes transform. The applications of these concepts and results to sample correlation matrix (SCM) models are presented in Section 2.5. In particular, the moments of an exponentially weighted and rectangulary windowed SCM are evaluated and the inverse of the SCM is characterized. Also, important results corresponding to the SCM of a white noise process are separately presented. Section 2.6 presents two important results that constitute the Gaussian method. This 
chapter is concluded with Section 2.7 which details how the asymptotic results are used and interpreted in practical applications.

\subsection{Limiting Eigenvalue Density Function}

The eigenvalues $\lambda_{1}, \lambda_{2}, \ldots, \lambda_{m}$ of an $m$-by- $m$ random and Hermitian matrix $\mathbf{A}_{m}$ can be encoded with a so-called empirical Eigenvalue Distribution Function $G^{\mathbf{A}_{m}}(x)$. This function is defined as the cumulative distribution function of the discrete uniform random variable which can take values equal to the eigenvalues of $\mathbf{A}_{m}$. That is,

$$
G^{\mathbf{A}_{m}}(x)=\frac{1}{m} \sum_{k=1}^{m} I_{(-\infty, x]}\left(\lambda_{k}\right),
$$

where $I_{[a, b]}(x)=1$ for $a \leq x \leq b$ and is zero otherwise.

The derivative of $G^{\mathbf{A}_{m}}(x)$ is the empirical Eigenvalue Density Function $\mu_{\mathbf{A}_{m}}(x)$, given by

$$
\mu_{\mathbf{A}_{m}}(x)=\frac{1}{m} \sum_{k=1}^{m} \delta_{D}\left(x-\lambda_{k}\right),
$$

where $\delta_{D}(x)$ is a Dirac delta function.

For some random matrix ensembles, when $m \rightarrow \infty$, the empirical Eigenvalue Density Function $\mu_{\mathbf{A}_{m}}(x)$ converges to a non-random limiting Eigenvalue Density Function $\mu_{\mathbf{A}}(x)$. The convergence is almost sure and the support of the limiting function is compact.

An example of a random matrix model whose empirical eigenvalue density function converges under some relatively mild conditions, often satisfied in practice, is a sample correlation matrix (SCM) corresponding to white noise process. Such a limiting eigenvalue density function is given in a closed form and is widely known as MarcenkoPastur law [33]. This result is presented in Section 2.5.3.

The SCM corresponding to white noise process is one of the few random matrix ensembles whose limiting Eigenvalue Density Function can be expressed in closed form. This is the motivation for introducing other ways of encoding the eigenvalues 
of random matrices.

\subsection{The Stieltjes Transform}

A possible way to encode the eigenvalues of a random matrix is via the Stieltjes transform. The Stieltjes transform is particularly useful in the theoretical analysis of adaptive processing with deficient sample support. This section defines the Stieltjes transform and presents important asymptotic characterizations used later in the analysis.

\subsubsection{The Eigenvalue and Eigenvector Stieltjes Transform}

In general, the Stieltjes transform $S(z)$ encodes a real-valued density function $\mu(x)$ such that

$$
S(z)=\int \frac{1}{x-z} \mu(x) d x
$$

where the integration is implied over the support of $\mu(x)$. The Stieltjes transform is formally defined for $\Im\{z\}>0$ ( $\{\{\}$ denotes the imaginary part of a complex number), so that the above integral does not have singularities.

The Stieltjes transform is intimately related to the Hilbert and Cauchy transform. Namely, the principal value of the scaled Stieltjes transform is the Hilbert transform. Also, the negative value of the Sieltjes transform is the Cauchy transform.

Given the Stieltjes transform $S(z)$, a density function $\mu(x)$ is easily recovered using the Stieltjes-Perron inversion formula [13]

$$
\mu(x)=\frac{1}{\pi} \lim _{\varepsilon \rightarrow 0} \Im\{S(x+i \varepsilon)\}
$$

\section{The Stieltjes Transform for Eigenvalues}

In the context of random matrix theory, the Stieltjes transform corresponding to the empirical Eigenvalue Density Function $\mu_{\mathbf{A}_{m}}(x)$ is, after substituting (2.2) into (2.3), 
given by

$$
S_{\mathbf{A}_{m}}(z)=\frac{1}{m} \sum_{k=1}^{m} \frac{1}{\lambda_{k}-z},
$$

where $z$ is a complex number with positive imaginary part. We refer to $S_{\mathbf{A}_{m}}(z)$ as the empirical Stieltjes transform. Recalling that the trace of a matrix is equal to the sum of its eigenvalues, $S_{\mathbf{A}_{m}}(z)$ is expressed as

$$
S_{\mathbf{A}_{m}}(z)=\frac{1}{m} \operatorname{tr}\left\{\left(\mathbf{A}_{m}-z \mathbf{I}\right)^{-1}\right\}
$$

For some random matrix ensembles, when $m \rightarrow \infty$, the empirical Stieltjes transform $S_{\mathbf{A}_{m}}(z)$ almost surely converges to a non-random limiting Stieltjes transform $\bar{S}_{\mathbf{A}}(z)$. A sample correlation matrix is a random matrix ensemble whose limiting Stieltjes transform exists under some relatively mild conditions, often satisfied in practice.

The limiting Stieltjes transform $\bar{S}_{\mathbf{A}}(z)$ is the Stieltjes transform (2.3) corresponding to the limiting Eigenvalue Density Function $\mu_{\mathbf{A}}(x)$. Therefore, $\mu_{\mathbf{A}}(x)$ can be obtained from $\bar{S}_{\mathbf{A}}(z)$ using the Stieltjes-Perron inversion formula (2.4). This is in fact the essence of the Stieltjes transform method [13].

The later developments exploit a simple relation between the Stieltjes transforms of two matrices related via linear transformation. Assuming a matrix $\mathbf{B}_{m}$ is obtained from $\mathbf{A}_{m}$ as

$$
\mathbf{B}_{m}=a \mathbf{A}_{m}+b \mathbf{I}
$$

where $a \neq 0$, the Stieltjes transforms $S_{\mathbf{B}_{m}}(z)$ and $S_{\mathbf{A}_{m}}(z)$ are related as

$$
S_{\mathbf{B}_{m}}(z)=\frac{1}{a} S_{\mathbf{A}_{m}}\left(\frac{z-b}{a}\right)
$$

This result straightforwardly follows from (2.6). In addition, if $S_{\mathbf{A}_{m}}(z)$ converges as $m \rightarrow \infty$, the relation between the limiting Stieltjes transforms remains the same.

The empirical and limiting Stieltjes transforms encode the corresponding densities of the eigenvalues. Therefore, we refer to these transforms as eigenvalue Stieltjes 
transforms. The Eigenvector Stieltjes Transform is introduced in the following section.

\section{The Stieltjes Transform for Eigenvectors}

Traditionally, the behavior of the eigenvectors of a random matrix is studied by analyzing the orthogonal projections. As such, it is known that the eigenvectors of the finite dimensional SCM corresponding to a Gaussian distributed, zero mean, unit power, white noise process are uniformly distributed on the unit sphere. In the effort to generalize this result without constraining the process be Gaussian, the projection of a random vector onto the subspace spanned by the eigenvectors of the SCM of white noise process is studied [13]. More recently, the angle between the eigenvector a random matrix and the ensemble eigenvector of the underlying process is asymptotically characterized in [6].

The eigenvectors of a random matrix are also studied implicitly via quadratic forms. This approach is more suitable for the applications considered in this thesis and is introduced here.

Given deterministic vectors $\mathbf{s}_{1}$ and $\mathbf{s}_{2}$, the eigenvalues and eigenvectors of a random matrix $\mathbf{A}_{m}$ are encoded via [21]

$$
F_{\mathbf{A}_{m}}(z)=\mathbf{s}_{1}^{H}\left(\mathbf{A}_{m}-z \mathbf{I}\right)^{-1} \mathbf{s}_{2}
$$

where $\Im\{z\} \neq 0$ so that $F_{\mathbf{A}_{m}}(z)$ has no singularities. We refer to function $F_{\mathbf{A}_{m}}(z)$ as the empirical Eigenvector Stieltjes Transform, although it depends on eigenvalues as well. Note that $F_{\mathbf{A}_{m}}(z)$ depends on the vectors $\mathbf{s}_{1}$ and $\mathbf{s}_{2}$. However, we omit showing this dependence explicitly in order to keep the notation uncluttered.

For some random matrix ensembles, when $m \rightarrow \infty$, the empirical Eigenvector Stieltjes Transform $F_{\mathbf{A}_{m}}(z)$ almost surely converges to a non-random limiting eigenvector Stieltjes transform $\bar{F}_{\mathbf{A}}(z)$. A sample correlation matrix is a random matrix ensemble whose limiting Stieltjes transform exists under some relatively mild conditions, often satisfied in practice.

The empirical eigenvector Stieltjes transforms corresponding to matrices $\mathbf{B}_{m}$ and 
$\mathbf{A}_{m}$ related via linear transformation

$$
\mathbf{B}_{m}=a \mathbf{A}_{m}+b \mathbf{I}
$$

where $a \neq 0$, are related as

$$
F_{\mathbf{B}_{m}}(z)=\frac{1}{a} F_{\mathbf{A}_{m}}\left(\frac{z-b}{a}\right)
$$

This result straightforwardly follows from (2.9). In addition, if $F_{\mathbf{A}_{m}}(z)$ converges as $m \rightarrow \infty$, the limiting eigenvector Stieltjes transforms are related in the same way.

\subsubsection{The Eigenvalue Stieltjes Transform for an important model}

The limiting Eigenvalue Stieltjes Transform of an important class of random matrices

is characterized with the following theorem [14]. The random matrix model considered in this theorem is essential for our study because it has a similar form to the SCM model used in the analysis.

Theorem 2.1. Let $m$ and $n$ be positive integers and let

$$
\mathbf{A}_{m}=\frac{1}{n} \mathbf{R}^{\frac{1}{2}} \mathbf{X} \mathbf{T} \mathbf{X}^{H} \mathbf{R}^{\frac{1}{2}}
$$

be an m-by-m matrix with the following hypothesis

1. $\mathbf{X}$ is an $m$-by-n matrix with i.i.d. complex entries of zero mean and unit variance.

2. $\mathbf{R}^{\frac{1}{2}}$ is an $m$-by-m Hermitian positive semi-definite square root of the positive semi-definite Hermitian matrix $\mathbf{R}$,

3. $\mathbf{T}=\operatorname{diag}\left(\tau_{1}, \ldots, \tau_{n}\right)$ with $\tau_{i} \geq 0$ for all positive integer-values $i$,

4. the sequences $\left\{G^{\mathbf{T}_{n}}(x)\right\}_{n=1}^{\infty}$ and $\left\{G^{\mathbf{R}_{m}}(x)\right\}_{m=1}^{\infty}$ are tight, i.e., for all $\varepsilon>0$, there exists $M>0$ such that $G^{\mathbf{T}}(M)>1-\varepsilon$ and $G^{\mathbf{R}}(M)>1-\varepsilon$ for all $n, m$. 
5. there exist $b>a>0$ for which $a<\liminf _{m \rightarrow \infty} c \leq \limsup _{m \rightarrow \infty} c<b$ with $c=m / n$.

Then, as $m$ and $n$ grow large with ratio c, the empirical Eigenvalue Stieltjes Transform of $\mathbf{A}_{m}, S_{\mathbf{A}_{m}}(z)$, almost surely converges to the limiting Eigenvalue Stieltjes Transform, $\bar{S}_{\mathbf{A}}(z)$, where

$$
\bar{S}_{\mathbf{A}}(z)=\frac{1}{m} \operatorname{tr}\left(\int \frac{\tau \mu_{\mathbf{T}}(\tau) d \tau}{1+c \tau e(z)} \mathbf{R}-z \mathbf{I}_{m}\right)^{-1}
$$

and the function $e(z)$ is the unique solution of the equation

$$
e(z)=\frac{1}{m} \operatorname{tr}\left\{\mathbf{R}\left(\int \frac{\tau \mu_{\mathbf{T}}(\tau) d \tau}{1+c \tau e(z)} \mathbf{R}-z \mathbf{I}_{m}\right)^{-1}\right\}
$$

such that the sign of the imaginary part of $e(z)$ and $z$ coincide if $\Im(z) \neq 0$ and $e(z)>0$ if $z$ is real and negative.

Note that the limiting Stieltjes transform $\bar{S}_{\mathbf{A}}(z)$ is expressed in terms of the function $e(z)$, which is given as the unique solution to the fixed point equation (2.14). The fixed point equation can be solved numerically, for example, by fixing $z$ and solving for $e(z)$ via classical fixed-point iterations. The accompanying theorem in [14] shows that the iterative algorithm converges if $e(z)$ is appropriately initialized. In particular, the theorem proves the convergence of the iterative method if $e(z)$ is initialized with $e^{(0)}(z)=-1 / z$.

A more general version of the theorem is stated and proved in [14]. The random matrix model considered therein is given as the sum of a Hermitian positive semidefinite matrix and sum of finite number of models of the same type as (2.12) with different and independent constituent matrices satisfying the same conditions as given in Theorem 2.1.

As a preview, $\mathbf{R}$ is the ensemble correlation matrix of the input to the adaptive processor. Also, the diagonal elements of $\mathbf{T}$ constitute windowing applied to the observation vectors. The examples of the ensemble correlation matrix corresponding to the input process consisting of a single or multiple plane waves impinging on an 
array of sensors are (3.113) and (3.117). Note that the inverse of the square root of the correlation matrix, $\mathbf{R}^{-\frac{1}{2}}$, is a whitening filter.

In the following, a couple of remarks are made regarding the conditions of the Theorem 2.1. First, the same characterization of the limiting Stieltjes transform holds if the entries in $\mathbf{X}$ are independent and have finite moments of order $2+\epsilon$ for some $\epsilon>0$. Note that this alleviates the necessity for having identically distributed entries in $\mathbf{X}$. The existence of moments of order higher than 2 implies that distributions of all entries in $\mathbf{X}$ have light tails ${ }^{1}[13]$.

Condition 3 requires that the matrix $\mathbf{T}$ be diagonal. The limiting Stieltjes transform for a more general version of the model which does not impose such a restriction on the matrix $\mathbf{T}$ is characterized in [65].

The sequences in condition 4 of Theorem 2.1 are the empirical eigenvalue distribution functions corresponding to matrices $\mathbf{T}$ and $\mathbf{R}$, and are by definition upper bounded by 1. Essentially, this means that with high increasingly high probability the eigenvalues of the matrices $\mathbf{T}$ and $\mathbf{R}$ do not blow out in the limit as $m \rightarrow \infty$.

As a final remark, note that in addition to taking the limit $m \rightarrow \infty$, which is in accordance with the definition of limiting Stieltjes transform, the number $n$ also grows large. Condition 5 implies that the ratio between $m$ and $n$ is finite and non-zero number, which further implies that $m$ and $n$ simultaneously grow and scale linearly.

\subsubsection{The Eigenvector Stieltjes Transform for an important model}

The limiting Eigenvector Stieltjes Transform of an important class of random matrices is characterized with the following theorem [21]. As in the previous part, the random matrix model considered in this theorem has a similar form to the SCM model used in the analysis.

\footnotetext{
${ }^{1}$ More specifically, it implies the Lindeberg-like condition [7]
} 
Theorem 2.2. Let $m$ and $n$ be positive integers and let

$$
F_{A_{m}}(z)=\mathbf{s}_{1}^{H}\left(\mathbf{A}_{m}-z \mathbf{I}\right)^{-1} \mathbf{s}_{2}
$$

be the empirical Eigenvector Stieltjes Transform corresponding to the $m$-by-m matrix $\mathbf{A}_{m}$

$$
\mathbf{A}_{m}=\frac{1}{n} \mathbf{R}^{\frac{1}{2}} \mathbf{X} \mathbf{X}^{H} \mathbf{R}^{\frac{1}{2}}
$$

Assume the following hypothesis hold

1. $\mathbf{s}_{1}$ and $\mathbf{s}_{2}$ are $m$-by-1 deterministic vectors with uniformly bounded norms for all $m$,

2. $\mathbf{X}$ is an $m$-by-n matrix with i.i.d. complex entries of zero mean, unit variance and finite eight-order moment,

3. $\mathbf{R}^{\frac{1}{2}}$ is an $m$-by-m Hermitian positive semi-definite square root of the positive semi-definite Hermitian matrix $\mathbf{R}$,

4. $\mathbf{R}$ has uniformly bounded spectral norm for all $m$, i.e., $\sup _{m}\|\mathbf{R}\|<\infty$.

Then, as $m$ and $n$ grow large at the same rate such that $\frac{m}{n} \rightarrow c$, where $c \in(0, \infty)$,

$$
|F(z)-\bar{F}(z)| \rightarrow 0
$$

almost surely for all z with $\Im\{z\}>0$. The limiting Eigenvector Stieltjes Transform is given by

$$
\bar{F}(z)=\sum_{i=1}^{m} \frac{\mathbf{s}_{1}^{H} \mathbf{q}_{i} \mathbf{q}_{i}^{H} \mathbf{s}_{2}}{\lambda_{i}\left(1-c-c z \bar{S}_{\mathbf{A}}(z)\right)-z},
$$

where $\bar{S}_{\mathbf{A}}(z)$ is the limiting Eigenvalue Stieltjes Transform corresponding to matrix $\mathbf{A}_{m}$ and $\lambda_{k}$ and $\mathbf{q}_{k}$ are the eigenvalues and eigenvectors of the matrix $\mathbf{R}$.

Note from the limiting result (2.18) that in general the eigenvectors are coupled with all eigenvalues via the Eigenvalue Stieltjes Transform $\bar{S}_{\mathbf{A}}(z)$. However, if $z \rightarrow$ $0^{-}$, each term in the sum (2.18) depends on one eigenvector and its corresponding 
eigenvalue. This decoupling effect is the implication of large $m, n$ limit in which all little perturbations, caused by a large number of random variables which compose the Eigenvector Stieltjes Transform, get combined in such a way that the variance of the quadratic form decreases.

Note that model (2.16) is a special case of (2.12) with $\mathbf{T}=\mathbf{I}$. In fact, the limiting Eigenvalue Stieltjes Transform of (2.16), $\bar{S}_{\mathbf{A}(z)}$, can be evaluated using Theorem 2.1 with $\mathbf{T}=\mathbf{I}$.

We point out that the same remark made with regard to condition 1 of Theorem 2.1 also holds here. Finally, note the equivalence between conditions 4 of Theorems 2.1 and 2.2 corresponding to matrix $\mathbf{R}$.

\subsection{Moments of Random Matrices}

The moments of a random matrix are quantities of our interest. This section defines the notion of a moment and elaborates how the moments can be computed from the Stieltjes transform.

The $k$-th empirical moment $M_{k}$ of a non-singular and Hermitian random matrix $\mathbf{A}_{m}$ is defined as a normalized trace of the $k$-th power of its inverse, namely

$$
M_{k}=\frac{1}{m} \operatorname{tr}\left\{\mathbf{A}_{m}^{-k}\right\}
$$

Note that empirical moment $M_{k}$ can be expressed in terms of the empirical eigenvalue density function $\mu_{\mathbf{A}_{m}}(x)$ as

$$
M_{k}=\int x^{-k} \mu_{\mathbf{A}_{k}}(x) d x .
$$

If the limiting Eigenvalue Density Function of a random matrix ensemble of interest exists, the empirical moment converges as $m \rightarrow \infty$ to a non-random quantity called limiting moment $\bar{M}_{k}$. The limiting moment is given as an expectation corre- 
sponding to the limiting Eigenvalue Density Function

$$
\bar{M}_{k}=\lim _{m \rightarrow \infty} \frac{1}{m} \operatorname{tr}\left\{\mathbf{A}_{m}^{-k}\right\}=\int x^{-k} \mu_{\mathbf{A}}(x) d x
$$

where the integration is implied over the support of $\mu_{\mathbf{A}}$.

The comparison between (2.6) and (2.19) reveals a relatively simple relation between the Eigenvalue Stieltjes Transform and first moment $M_{1}$. Although the empirical Stieltjes transform is formally defined for non-real $z$ (in order to avoid singularities), its support is extended to real $z$ which do not fall inside the support of the underlying eigenvalue density function. Assuming the sequence of matrices $\mathbf{A}_{m}$ is positive definite, $S_{\mathbf{A}_{m}}(z)$ is defined for $z$ such that $\operatorname{Im}\{z\}=0$ and $\operatorname{Re}\{z\} \rightarrow 0^{-}$, which we compactly represent as $z \rightarrow 0^{-}$. Hence, using (2.6) the empirical moment $M_{1}$ is given by

$$
M_{1}=\lim _{z \rightarrow 0^{-}} S_{\mathbf{A}_{m}}(z)
$$

Similarly, the limiting first moment $\bar{M}_{1}$ is related to the limiting eigenvalue Stieltjes transform $S(z)$ (if it exists) via

$$
\bar{M}_{1}=\lim _{z \rightarrow 0^{-}} \bar{S}_{\mathbf{A}}(z)
$$

The higher order empirical and limiting moments can be obtained similarly from the empirical and limiting Stieltjes transforms. The $k$-th derivative with respect to $z$ of the empirical Eigenvalue Stieltjes Transform (2.6) is given by

$$
\frac{\partial^{k} S_{\mathbf{A}_{m}}(z)}{\partial z^{k}}=\frac{k !}{m} \operatorname{tr}\left\{\left(\mathbf{A}_{m}-z \mathbf{I}\right)^{-(k+1)}\right\}
$$

Assuming the sequence of matrices $\mathbf{A}_{m}$ is positive definite and order $k$ is finite, the $k$-th derivative of $S_{\mathbf{A}_{m}}(z)$ is defined as $z \rightarrow 0^{-}$. Thus, the empirical $k$-th moment $M_{k}$ is obtained from the $k$-th derivative of the empirical eigenvalue Stieltjes transform $S_{\mathbf{A}_{m}}(z)$ as

$$
M_{k}=\frac{1}{k !} \lim _{z \rightarrow 0^{-}} \frac{\partial^{k} S_{\mathbf{A}_{m}}(z)}{\partial z^{k}}
$$


Finally, the limiting $k$-th moment $\bar{M}_{k}$ is given from the $k$-th derivative of the limiting Eigenvalue Stieltjes Transform by

$$
\bar{M}_{k}=\frac{1}{k !} \lim _{z \rightarrow 0^{-}} \frac{\partial^{k} \bar{S}_{\mathbf{A}}(z)}{\partial z^{k}}
$$

This method is used to evaluate the limiting moments needed for the performance analysis of adaptive processors operating in the deficient sample support regime. As a final note, in a more general framework, other important quantities in random matrix theory are characterized using the Stieltjes transform and similar methods [22]. The obtained characterizations are commonly known as Girko estimators.

\subsection{Sample Correlation Matrix Models}

The models of the sample correlation matrices used in this thesis are introduced in this section. Also, the limiting Stieltjes transforms corresponding to these models are characterized and the limiting moments are evaluated. The limiting eigenvalue density functions of two SCM models corresponding to white noise process are presented in the last part.

\subsubsection{The Moments of the Exponentially Weighted SCM}

The exponentially weighted SCM computed at discrete time $n$ from observation vectors $\mathbf{u}(k)$ of dimension $m$, received at discrete times $k=1,2, \ldots, n$, is defined as

$$
\hat{\mathbf{R}}(n)=\sum_{k=1}^{n} \lambda^{n-k} \mathbf{u}(k) \mathbf{u}^{H}(k)
$$

where $\lambda \in(0,1)$ is an exponential forgetting factor. The exponential forgetting factor $\lambda$ attenuates past observations not relevant for the estimate of correlation matrix at current time instant. The forgetting factor $\lambda$ is usually very close to 1 .

Note that the effective number of observations is controlled by the value of forgetting factor $\lambda$. That is, if $\lambda$ is small, only few most recent observations impact the 
SCM (2.27). A common rule of thumb is that the effective number of observations $n_{\mathrm{eff}}$ is $1 /(1-\lambda)[27]$.

The effective number of observations is a relevant quantity in the study of adaptive processing with deficient sample support when the exponential weighting of observation vectors is employed.

\section{Random Matrix Model for the SCM}

If the ensemble correlation matrix of the arrival process is $\mathbf{R}$, the observation vector $\mathbf{u}(k)$ is modeled as a colored process

$$
\mathbf{u}(k)=\mathbf{R}^{\frac{1}{2}} \mathbf{x}(k)
$$

where $\mathbf{R}^{\frac{1}{2}}$ is a positive-semi definite square root of the correlation matrix $\mathbf{R}$ and $\mathbf{x}(k) \in \mathbb{C}^{m}$ is a vector of i.i.d. zero mean, unit variance entries.

From (2.28) and (2.27), the exponentially weighted SCM can be compactly represented by

$$
\hat{\mathbf{R}}(n)=\mathbf{R}^{\frac{1}{2}} \mathbf{X} \boldsymbol{\Lambda}(n) \mathbf{X}^{H} \mathbf{R}^{\frac{1}{2}},
$$

where

1. $\mathbf{X}$ is $m$-by- $n$ matrix whose $k$-th column, $\mathbf{x}(k)$, is an observation vector of a zero mean, unit power white noise and

2. $\boldsymbol{\Lambda}(n)=\operatorname{diag}\left(\lambda^{n-1}, \lambda^{n-2}, \ldots, 1\right)$ is a diagonal matrix of order $n$ composed from the powers of forgetting factor $\lambda$.

\section{The Limiting First Moment of the Exponentially Weighted SCM}

In the following, the limiting first moment $\bar{M}_{1}$ corresponding to the exponentially weighted SCM is evaluated. As elaborated in Section 2.4, the first step is to characterize the limiting Eigenvalue Stieltjes Transform. This is done by using the result of Theorem 2.1. Then, the limiting first moment is obtained from the limiting Stieltjes transform by taking the limit $z \rightarrow 0^{-}$. 
The exponentially weighted SCM (2.29) is equivalent to the matrix $\mathbf{A}_{m}$ (2.12) up to scaling when $\mathbf{T}=\mathbf{I}$. Namely,

$$
\mathbf{A}_{m}=\frac{1}{n} \hat{\mathbf{R}}(n)
$$

Their limiting Eigenvalue Stieltjes Transforms are using (2.8) related as

$$
\bar{S}_{\mathbf{A}}(z)=n \bar{S}_{\hat{\mathbf{R}}}(n z)
$$

where $\bar{S}_{\hat{R}}(z)$ is the limiting eigenvalue Stieltjes transform corresponding to the exponentially weighted $\operatorname{SCM} \hat{\mathbf{R}}(n)$.

The empirical Eigenvalue Density Function of $\boldsymbol{\Lambda}(n)$ is given by

$$
\mu_{\Lambda(n)}(\tau)=\frac{1}{n} \sum_{k=1}^{n} \delta_{D}\left(\tau-\lambda^{n-k}\right)
$$

The substitution of (2.30) and (2.32) into (2.13) yields the characterization of the limiting Eigenvalue Stieltjes Transform of SCM $\hat{\mathbf{R}}$

$$
n \bar{S}_{\hat{\mathbf{R}}}(n z)=\frac{1}{m} \operatorname{tr}\left(\frac{1}{n} \sum_{k=1}^{n} \frac{\lambda^{n-k}}{1+c \lambda^{n-k} e(z)} \mathbf{R}-z \mathbf{I}\right)^{-1}
$$

where $e(z)$ is obtained by substituting (2.32) into (2.14) as

$$
e(z)=\frac{1}{m} \operatorname{tr}\left\{\mathbf{R}\left(\frac{1}{n} \sum_{k=1}^{n} \frac{\lambda^{n-k}}{1+c \lambda^{n-k} e(z)} \mathbf{R}-z \mathbf{I}\right)^{-1}\right\}
$$

Taking the limit $z \rightarrow 0^{-}$(i.e., $\Im\{z\}=0$ and $\Re\{z\} \rightarrow 0^{-}$) in (2.34) and denoting $e(0)=\lim _{z \rightarrow 0^{-}} e(z)$ yields

$$
\frac{1}{e(0)}=\frac{1}{n} \sum_{k=1}^{n} \frac{\lambda^{n-k}}{1+c \lambda^{n-k} e(0)}
$$


Similarly, taking the limit $z \rightarrow 0^{-}$in $(2.33)$ yields

$$
n \bar{M}_{1}=\frac{1}{m}\left(\frac{1}{n} \sum_{k=1}^{n} \frac{\lambda^{n-k}}{1+c \lambda^{n-k} e(0)}\right)^{-1} \operatorname{tr}\left\{\mathbf{R}^{-1}\right\} .
$$

We observe from (2.35) and (2.36) that $n \bar{M}_{1}=\frac{1}{m} \operatorname{tr}\left\{\mathbf{R}^{-1}\right\} e(0)$ and solve for $e(0)$ in terms of $\bar{M}_{1}$. After substituting the result in (2.35) and rearranging the obtained expression, the limiting first moment is finally given as a fixed point solution to

$$
\frac{1}{\bar{M}_{1}}=\sum_{k=1}^{n} \frac{\lambda^{n-k}}{\frac{1}{m} \operatorname{tr}\left\{\mathbf{R}^{-1}\right\}+m \lambda^{n-k} \bar{M}_{1}}
$$

Recall that the fixed-point iterative method, if appropriately initialized, converges to the unique solution of the fixed point equation (2.14). Since the fixed point equation (2.35) is obtained from (2.14) by taking the limit $z \rightarrow 0^{-}$, we conclude that $(2.35)$ has unique solution which can be found by employing the iterative algorithm. Consequently, the limiting moment $\bar{M}_{1}$ is given as the unique solution to the fixed-point equation (2.37).

Given that the iterative algorithm initialized with $e^{(0)}(z)=-1 / z$ converges to the unique solution of (2.14) [14], the iterative procedures for solving (2.35) and (2.37) should be initialized with a large negative number. However, since the solutions to (2.35) and (2.37) are positive reals, an initialization with a small positive number suffices. The simulations performed to validate the results in later chapters confirm this observation.

Note that in a special case when $\lambda=1$, the limiting moment $\bar{M}_{1}$ is, using (2.37), given in a closed form

$$
\bar{M}_{1}=\frac{1}{m(n-m)} \operatorname{tr}\left\{\mathbf{R}^{-1}\right\}
$$




\subsubsection{The Moments and Inverse of the Rectangularly Win- dowed SCM}

The SCM evaluated from $n$ rectangularly windowed observation vectors of dimension $m$ has been introduced in Section 1.1.2. For completeness we repeat the definition here. Namely, given the stationary observation vectors $\mathbf{u}(k)$ at discrete times $k=$ $1,2, \ldots, n$, the rectangularly windowed SCM is given by

$$
\hat{\mathbf{R}}=\frac{1}{n} \sum_{k=1}^{n} \mathbf{u}(k) \mathbf{u}^{H}(k) .
$$

As already elaborated, if the ensemble correlation matrix of the arrival pro-

cess is $\mathbf{R}$, the observation vector $\mathbf{u}(k)$ is modeled using (2.28). Substituting (2.28) into (2.39), the rectangularly weighted SCM is compactly represented as

$$
\hat{\mathbf{R}}=\frac{1}{n} \mathbf{R}^{\frac{1}{2}} \mathbf{X} \mathbf{X}^{H} \mathbf{R}^{\frac{1}{2}},
$$

where $\mathbf{X}$ is $m$-by- $n$ matrix of i.i.d. zero mean, unit variance entries. Its columns model observations of a zero mean, unit power white noise process.

The SCM in (2.39) is usually diagonally loaded in order to improve the condition number of the resulting matrix and for some additional reasons that will become clear in Chapter 3. The diagonally loaded SCM is given by

$$
\hat{\mathbf{R}}_{\delta}=\hat{\mathbf{R}}+\delta \mathbf{I},
$$

where $\delta>0$ is a diagonal loading parameter.

The limiting eigenvalue Stieltjes transforms of $\hat{\mathbf{R}}$ and $\hat{\mathbf{R}}_{\delta}$ are characterized in the following part. 


\section{The Limiting Eigenvalue Stieltjes Transform of the SCM}

The limiting eigenvalue Stieltjes transforms corresponding to the unloaded SCM $\hat{\mathbf{R}}$ and diagonally loaded SCM $\hat{\mathbf{R}}_{\delta}$ are from (2.8) related as

$$
\bar{S}_{\hat{\mathbf{R}}_{\delta}}(z)=\bar{S}_{\hat{\mathbf{R}}}(z-\delta) .
$$

The limiting Stieltjes transform $\bar{S}_{\hat{\mathbf{R}}}(z)$ is found using Theorem 2.1 by setting $\mathbf{T}=\mathbf{I}$ in (2.12). Since $\mu_{\mathbf{T}}(\tau)=\delta_{D}(\tau-1)$, the Stieltjes transform $\bar{S}_{\hat{\mathbf{R}}}(z)$ is using (2.13) expressed as

$$
\begin{aligned}
\bar{S}_{\hat{\mathbf{R}}}(z) & =\frac{1}{m} \operatorname{tr}\left(\int \frac{\tau \delta_{\mathrm{D}}(\tau-1) d \tau}{1+c \tau e(z)} \mathbf{R}-z \mathbf{I}_{m}\right)^{-1} \\
& =\frac{1}{m} \sum_{k=1}^{m} \frac{1}{(1+c e(z))^{-1} \lambda_{k}-z}
\end{aligned}
$$

where $e(z)$ is the solution to a fixed point equation (2.14) expressed as

$$
\begin{aligned}
e(z) & =\frac{1}{m} \operatorname{tr}\left\{\mathbf{R}\left(\int \frac{\tau \delta_{\mathrm{D}}(\tau-1) d \tau}{1+c \tau e(z)} \mathbf{R}-z \mathbf{I}_{m}\right)^{-1}\right\} \\
& =\frac{1}{m} \sum_{k=1}^{m} \frac{\lambda_{k}}{(1+c e(z))^{-1} \lambda_{k}-z}
\end{aligned}
$$

To derive a more compact representation of $\bar{S}_{\hat{\mathbf{R}}}(z)$, we introduce a new variable $t(z)=1+c e(z)$ which, using (2.44), is given by

$$
t(z)=1+\frac{c}{m} \sum_{k=1}^{m} \frac{t(z) \lambda_{k}}{\lambda_{k}-z t(z)}
$$

Equivalently,

$$
t^{-1}(z)=1-\frac{c}{m} \sum_{k=1}^{m} \frac{\lambda_{k}}{\lambda_{k}-z t(z)} .
$$

The Stieltjes transform $\bar{S}_{\hat{\mathbf{R}}}(z)$ is, using (2.43), expressed in terms of $t(z)$ as

$$
\bar{S}_{\hat{\mathbf{R}}}(z)=\frac{1}{m} \sum_{k=1}^{m} \frac{t(z)}{\lambda_{k}-z t(z)} .
$$


Rearranging the summand in (2.46) and using the above expression yields

$$
t^{-1}(z)=1-c-c z \bar{S}_{\hat{\mathbf{R}}}(z)
$$

Finally, the limiting Stieltjes transform corresponding to the SCM $\hat{\mathbf{R}}$ is obtained after substituting (2.48) into (2.47) and is given by

$$
\bar{S}_{\hat{\mathbf{R}}}(z)=\frac{1}{m} \sum_{k=1}^{m} \frac{1}{\left(1-c-c z \bar{S}_{\hat{\mathbf{R}}}(z)\right) \lambda_{k}-z} .
$$

Note the similarity in the forms of (2.49) and (2.18). In addition, (2.49) could be easily obtained from the characterization of the limiting Stieltjes transform given in [21] and after a bit of algebraic manipulations derived from the characterization given in [65].

The limiting Stieltjes transform corresponding to a diagonally loaded SCM $\hat{\mathbf{R}}_{\delta}$ is using (2.42) and (2.49) given as the solution to

$$
\bar{S}_{\hat{\mathbf{R}}_{\delta}}(z)=\frac{1}{m} \sum_{k=1}^{m} \frac{1}{\left(1-c-c(z-\delta) \bar{S}_{\hat{\mathbf{R}}_{\delta}}(z)\right) \lambda_{k}-z+\delta} .
$$

Note that the existence and uniqueness of the solution to the above fixed point equation follows from the existence and uniqueness of the solution to (2.14). In addition, the fixed-point iterative procedure converges to the solution for appropriate initialization.

\section{The Limiting Moments of the Diagonally Loaded SCM}

The limiting moments $\bar{M}_{1}$ and $\bar{M}_{2}$ corresponding to a diagonally loaded SCM $\hat{\mathbf{R}}_{\delta}$ are evaluated in this part. These results are exploited in the theoretical analysis in Section 3.5.

As elaborated in Section 2.4, the limiting moment $\bar{M}_{1}$ is obtained by taking the 
limit $z \rightarrow 0^{-}$in (2.50) and is given by the solution to a fixed point equation

$$
\bar{M}_{1}=\frac{1}{m} \sum_{k=1}^{m} \frac{1}{\left(1-c+c \delta \bar{M}_{1}\right) \lambda_{k}+\delta} .
$$

Note that (2.38) and (2.51) for $\delta=0$ are identical up to a scaling factor $n$ because the SCM corresponding to (2.38) for $\lambda=1$ is not normalized with $n$.

The limiting moment $\bar{M}_{2}$ is evaluated, using (2.26), by taking the limit $z \rightarrow 0^{-}$

of the first derivative of $\bar{S}_{\hat{\mathbf{R}}_{\delta}}(z)$ with respect to $z$. After few algebraic steps omitted here, the moment $\bar{M}_{2}$ is given as the solution to

$$
\bar{M}_{2}=\frac{1}{m} \sum_{k=1}^{m} \frac{c\left(\bar{M}_{1}-2 \delta \bar{M}_{2}\right) \lambda_{k}+1}{\left(\left(1-c+c \delta \bar{M}_{1}\right) \lambda_{k}+\delta\right)^{2}}
$$

Given that the solution to the fixed point equation (2.50) exists and is unique, the solutions to (2.51) and (2.52) exist and are unique. These fixed point equations can be solved via the classical iterative method which converges if appropriately initialized. Given that the considered SCM is diagonally loaded with $\delta$, we initialize the iterative methods with respectively $1 / \delta$ and $1 / \delta^{2}$.

\section{The Inverse of the Rectangularly Windowed SCM}

This part characterizes the inverse of the SCM (2.39) evaluated from $n$ rectangulary windowed observation vectors of dimension $m$. Assuming the observation vectors have ensemble correlation matrix $\mathbf{R}$, the SCM is modeled as in (2.40). The limiting Eigenvector Stieltjes Transform for this model is characterized in Theorem 2.2.

Since the probability of receiving two observation vectors that are identical up to a scaling is zero, the SCM $\hat{\mathbf{R}}$ is non-singular when $n>m{ }^{2}$ Therefore, the support of the empirical Eigenvector Stieltjes Transform (2.9) can be extended to include $z$ with $\Im\{z\}=0$ and $\Re\{z\} \leq 0$. Taking the limit $z \rightarrow 0^{-}$(i.e., $\Im\{z\}=0$ and $\Re\{z\} \rightarrow 0^{-}$)

\footnotetext{
${ }^{2}$ Note however that two observation vectors can be close to each other at very high SNR's leading to an ill-conditioned SCM.
} 
in (2.17) and (2.18) yields that as $m, n \rightarrow \infty$ such that $m / n \rightarrow c \in(0,1)$,

$$
\mathbf{s}_{1}^{H} \hat{\mathbf{R}}^{-1} \mathbf{s}_{2} \rightarrow \sum_{k=1}^{m} \frac{\mathbf{s}_{1}^{H} \mathbf{q}_{k}^{H} \mathbf{q}_{k} \mathbf{s}_{2}}{\lambda_{k}(1-c)} \quad \text { a.s. }
$$

where $\lambda_{k}$ and $\mathbf{q}_{k}$ are the eigenvalues and eigenvectors of the ensemble correlation matrix $\mathbf{R}$ and $\mathbf{s}_{1}$, and $\mathbf{s}_{2}$ are non-zero deterministic vectors with uniformly bounded norms.

Noting that the limiting quantity in (2.53) is the quadratic form corresponding to the inverse of the ensemble correlation matrix $\mathbf{R}$ scaled by $1 /(1-c)$, it is finally concluded that as $m, n \rightarrow \infty$ such that $m / n \rightarrow c \in(0,1)$,

$$
\hat{\mathbf{R}}^{-1} \rightarrow \frac{1}{1-c} \mathbf{R}^{-1} \quad \text { a.s. }
$$

\subsubsection{White Noise Process}

This part summarizes results concerning the limiting eigenvalue density functions corresponding to exponentially weighted and rectangularly windowed SCM of white noise process.

\section{Rectangularly Windowed SCM}

The SCM of $n$ rectangularly weighted observation vectors of zero mean, unit variance white noise of dimension $m$ is modeled $\mathrm{as}^{3}$

$$
\hat{\mathbf{R}}=\frac{1}{n} \mathbf{X X}^{H}
$$

where $\mathbf{X}$ is $m$-by- $n$ matrix with i.i.d. zero mean, unit variance entries.

The limiting Stieltjes transform corresponding to (2.55) is obtained from Theorem 2.1 with $\mathbf{T}=\mathbf{I}$ and $\mathbf{R}=\mathbf{I}$. Employing the Perron-Stieltjes inversion formula (2.4), the corresponding limiting Eigenvalue Density Function $\mu_{\hat{\mathbf{R}}}(x)$ is, in the

\footnotetext{
${ }^{3}$ The cases of input processes of non-unit variance are easily accommodated by including proper scaling.
} 
limit when $m, n(m) \rightarrow \infty$ at the same rate such that $m / n \rightarrow c \in(0, \infty)$, obtained in a closed form as

$$
\mu_{\hat{\mathbf{R}}}(x)=\max \left(1-\frac{1}{c}, 0\right) \delta_{D}(x)+\frac{\sqrt{(x-a)(b-x)}}{2 \pi c x} I_{[a, b]}(x),
$$

where $I_{[a, b]}(x)=1$ for $a \leq x \leq b$ and is zero otherwise. The support of this function is compact with endpoints $a=(1-\sqrt{c})^{2}$ and $b=(1+\sqrt{c})^{2}$. In addition, all the eigenvalues of (2.55) almost surely fall within the support of $\mu_{\hat{\mathbf{R}}}(x)$ in the limit $m, n(m) \rightarrow \infty$ [4]. Finally, note a non-zero mass at 0 when $\hat{\mathbf{R}}$ is not full-rank.

This result is widely known as the Marcenko-Pastur law [33]. Some other versions of the law, which do not require identically distributed entries in $\mathbf{X}$ are reviewed in [54]. Essentially, the condition that entries in $\mathbf{X}$ are identically distributed can be abandoned at the expense of imposing the requirement that the entries have finite moments of order $2+\epsilon$ for some $\epsilon>0$ [13].

The plots of Marcenko-Pastur law (2.56) for different values of parameter $c$ are shown in Fig. 2-1. Note that the ensemble eigenvalue of the considered process is 1 (of multiplicity $m$ ). Recall that $c^{-1}$ represents the average number of observations per dimension. Therefore, it is intuitively clear that as $c$ decreases, i.e., as more observations per dimension become available, the eigenvalues of the SCM concentrate around the ensemble eigenvalue. In other words, the SCM more accurately estimates the ensemble correlation matrix.

As a final remark, it can be noted from Fig. 2-1 that the limiting Eigenvalue Density Function is not symmetric around the ensemble eigenvalue. More specifically, the plots show relatively high probability of observing an eigenvalue smaller than 1. This implies that the SCM might not be well conditioned when $n$ is not enough times larger than $m$. We will return to this observation in Section 4.5.3.

\section{The Moments of the SCM of White Noise}

The moments corresponding to the SCM of white noise process can be evaluated from the limiting Eigenvalue Density Function (2.56) using (2.20). 


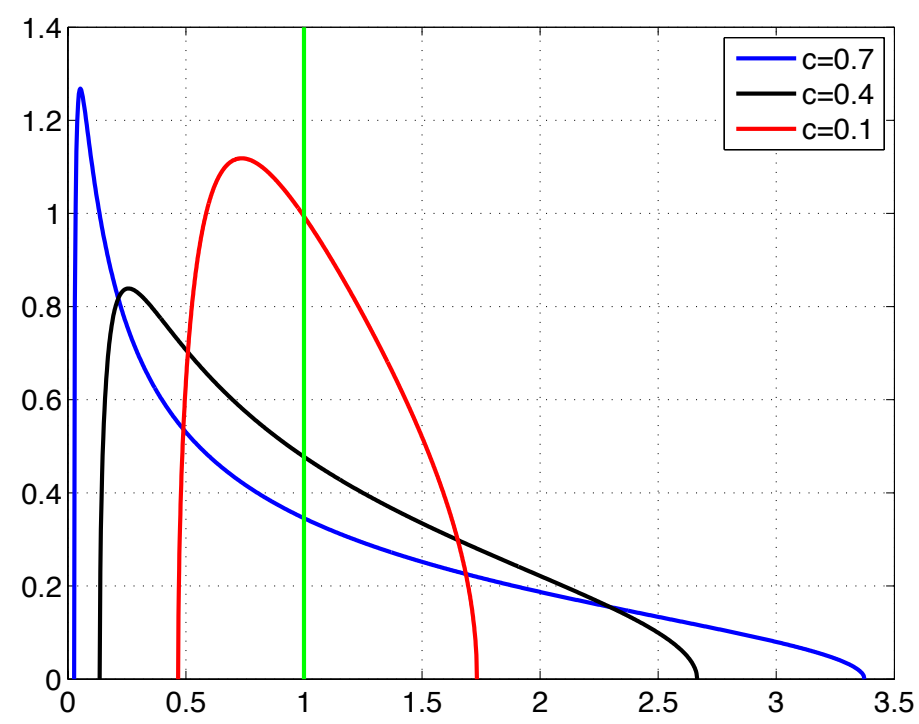

Figure 2-1: The limiting Eigenvalue Density Function corresponding to SCM of zero mean, unit variance white noise process for different values of parameter $c$. The Eigenvalue Density Function corresponding to the ensemble correlation matrix is $\delta_{D}(x-1)$ (shown in green).

For the reasons that will become clear in Chapter 4, we are interested in computing the limiting moments $\bar{M}_{1}$ and $\bar{M}_{2}$ of a model

$$
\Phi=n \hat{\mathbf{R}}+\delta \mathbf{I}
$$

where $\hat{\mathbf{R}}$ is defined in $(2.55)$.

Since the eigenvalues of $\boldsymbol{\Phi}$ and the rectangularly windowed SCM $\hat{\mathbf{R}}$ are related through

$$
\lambda_{k}(\boldsymbol{\Phi})=n \lambda_{k}(\hat{\mathbf{R}})+\delta, \quad k=1,2, \ldots, m
$$

the corresponding limiting eigenvalue density functions are related as

$$
\mu_{\boldsymbol{\Phi}}(x)=\frac{1}{n} \mu_{\hat{\mathbf{R}}}\left(\frac{x-\delta}{n}\right) .
$$

Using (2.56), (2.59), and (2.20), the limiting moments $M_{1}$ and $M_{2}$ are evaluated 
in closed form and given by

$$
\bar{M}_{1}=\max \left(0,1-\frac{1}{c}\right) \frac{1}{\delta}+\frac{\sqrt{\delta^{2}+(m-n)^{2}+2 \delta(m+n)}-|n-m|-\delta}{2 \delta m}
$$

and

$$
\bar{M}_{2}=\max \left(0,1-\frac{1}{c}\right) \frac{1}{\delta^{2}}-\frac{|n-m|}{2 \delta^{2} m}+\frac{(m-n)^{2}+\delta(m+n)}{2 \delta^{2} m \sqrt{\delta^{2}+(m-n)^{2}+2 \delta(m+n)}} .
$$

\section{Exponentially Weighted SCM}

Here we consider another version of a scaled SCM. It is computed from $n$ observations of zero mean, unit variance white noise process whose observation vectors are exponentially weighted with forgetting factor $\lambda$ as

$$
\boldsymbol{\Phi}=(1-\lambda) \mathbf{X} \mathbf{\Lambda} \mathbf{X}^{H}
$$

where $\mathbf{X}$ is $m$-by- $n$ matrix of i.i.d. zero mean, unit variance entries and $\boldsymbol{\Lambda}=$ $\operatorname{diag}\left(\lambda^{n-1}, \ldots, \lambda, 1\right)$. Note that $\boldsymbol{\Phi}$ is a consistent estimator in the limit as $n \rightarrow \infty$ when $m$ and $\lambda$ are finite and fixed.

The limiting Stieltjes transform corresponding to (2.62) can be characterized using Theorem 2.1 with $\mathbf{T}=\boldsymbol{\Lambda}$ and accounting for scaling factor $1-\lambda$. It is shown that

as $n, m, 1 /(1-\lambda) \rightarrow \infty$ at a non-zero, finite rate $q=m(1-\lambda) \in(0, \infty)$ the limiting Stieltjes transform of $\boldsymbol{\Phi}$ is given as the unique solution to [9]

$$
z q \bar{S}_{\boldsymbol{\Phi}}(z)=q-\log \left(1-q \bar{S}_{\Phi}(z)\right)
$$

As discussed with regard to Theorem 2.1, (2.63) can be solved via fixed-point iterations, which, if appropriately initialized, converge to the unique solution. The limiting eigenvalue density function $\mu_{\boldsymbol{\Phi}}(x)$ of the scaled and exponentially weighted SCM (2.62) is then obtained from the inversion formula (2.4). Note that the parameter $q$ represents the effective average number of observations per dimension. In that, it is analogous to the parameter $c$ in the Marcenko-Pastur law. 
The limiting eigenvalue density $\mu_{\boldsymbol{\Phi}}(x)$ (obtained from (2.63) and (2.4)) has compact support whose upper and lower edges $x_{1}$ and $x_{2}$ are the solutions to [9]

$$
x=\log x+q+1
$$

As a final remark, we point out that the plots of the limiting eigenvalue density functions of the rectangularly windowed (Marcenko-Pastur law) and exponentially weighted SCM look alike for appropriately chosen parameters $c$ and $q[9]$. This observation is exploited in the analysis in Section 4.7.1.

\subsection{Gaussian Method for Random Matrices}

The random matrix theory concepts discussed so far focus on defining quantities that encode the eigenvalues and eigenvectors of a random matrix and proving their convergence to non-random limits. The stated results provide characterizations of the limiting eigenvalue and eigenvector Stieltjes transforms for a class of models that is particularly useful in describing an SCM of an arrival process. These characterizations hold under relatively mild conditions on input data statistics without restricting the input data to originate from any specific probability distribution. In addition, the results prove the convergence to non-random limits without specifying the rates of convergence.

As opposed to that approach, the Gaussian method requires the arrival process be Gaussian distributed. In addition, the rates of convergence of different quantities which encode the eigenvalues and eigenvectors naturally follow from the analysis. To the best of our knowledge, the Gaussian method has been introduced for the first time to study the behavior of mutual information in a Gaussian MIMO channel [25].

The Gaussian method is based on two important results which address the expectation and variance of functionals of Gaussian random variables. These results, called Gaussian tools, are stated here and applied in Section 3.6. 
We assume that a $m$-by- $n$ random matrix $\mathbf{Y}$ is modeled as

$$
\mathbf{Y}=\mathbf{D}^{\frac{1}{2}} \mathbf{X}
$$

where

1. $\mathbf{D}^{\frac{1}{2}}$ is the $m$-by- $m$ positive semi-definite square root of the non-negative definite diagonal matrix $\mathbf{D}$ with uniformly bounded diagonal elements.

2. $\mathbf{X}$ is an $m$-by- $n$ matrix with i.i.d. complex Gaussian entries of zero mean and unit variance.

Note that the columns of the matrix $\mathbf{Y}$ model the observation vectors of Gaussian distributed input process whose eigenvalues are the diagonal elements of the matrix D.

An integration by parts formula characterizes the expectation of a functional of a Gaussian matrix Y modeled as (2.65) [25]

$$
\mathbf{E}\left[Y_{i j} f(\mathbf{Y})\right]=d_{i} \mathbf{E}\left[\frac{\partial f(\mathbf{Y})}{\partial Y_{i j}^{*}}\right]
$$

where $d_{i}$ is the $i$-th diagonal element of the matrix $\mathbf{D}, f(\mathbf{Y})$ is a functional of a Gaussian matrix $\mathbf{Y}$ whose $(i, j)$ entry is $Y_{i j}$ and $Y_{i j}^{*}$ is a complex-conjugate of $Y_{i j}$. This result is used in order to evaluate the expectations of different quadratic forms.

The Poincare-Nash inequality upper bounds the variance of a functional of a Gaussian matrix Y modeled as (2.65) [25]

$$
\operatorname{var}(f(\mathbf{Y})) \leq \sum_{i=1}^{m} \sum_{j=1}^{n} d_{i} \mathbf{E}\left[\left|\frac{\partial f(\mathbf{Y})}{\partial Y_{i j}}\right|^{2}+\left|\frac{\partial f(\mathbf{Y})}{\partial Y_{i j}^{*}}\right|^{2}\right]
$$

This result is used in Chapter 3 to prove that the variances of different functionals are upper bounded by quantities which decay to zero in the limit when $m$ and $n$ grow large at the same rate. Applying the Cauchy-Schwartz inequality, different functionals are then proved to be approximately uncorrelated. 


\subsection{Interpretation of Random Matrix Theory Re- sults}

The random matrix theory results and concepts presented in the previous sections are asymptotic in nature. That is, the number of dimensions $m$ and the number of observations $n$ used to compute the SCM grow large and scale linearly such that $m / n \rightarrow c \in(0, \infty)$. Since the numbers of dimensions and observations in all practical scenarios are finite, a natural question of how to exploit the random matrix theory results arises.

Before answering this question, the following numerical experiment is conducted. Suppose $m$ sensors receive i.i.d. observation vectors of zero mean, unit variance white noise process. A number of realizations of SCM's, each computed from $n$ different rectangularly windowed observation vectors are generated. Then, a set of eigenvalues is formed either by collecting all the eigenvalues computed from each realization of the SCM, or by sampling uniformly at random an eigenvalue from each realization of an SCM. In the final step, a histogram of the obtained collection of eigenvalues is computed and normalized such that its area is 1 . The normalized histogram is shown in Fig. 2-2 for $n=20$ and $m=10$. Note that these values of $m$ and $n$ imply that the SCM is evaluated with 2 observations per dimension, i.e., $c=0.5$. The limiting Eigenvalue Density Function corresponding to this arrival model is characterized with the Marcenko-Pastur law (2.56). The plot of this function parameterized with $c=0.5$ is also shown in Fig. 2-2. Note that the Marcenko-Pastur law (i.e., more generally,

the limiting Eigenvalue Density Function) corresponds to a single realization of the SCM in the large $m$ and $n$ limit, as opposed to a histogram which is evaluated from a number of SCM realizations.

The agreement between the normalized histogram and Marcenko-Pastur law in Fig. 2-2 implies that even though the limiting Eigenvalue Density Function is the asymptotic result obtained when $m, n \rightarrow \infty$ such that $m / n \rightarrow c$, where $c=0.5$, it fairly accurately approximates the expectation of the distribution when $m$ and $n$ are finite and relatively small. 


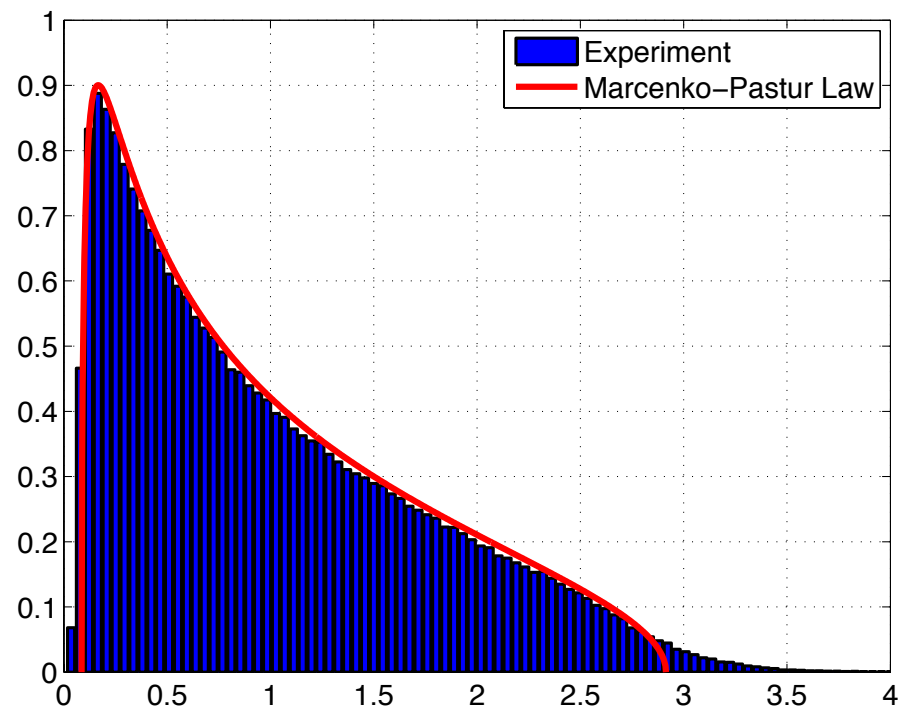

Figure 2-2: Comparison between Marcenko-Pastur law with $c=0.5$ and experimentally obtained Eigenvalue Density Function for $n=20$ and $m=10$.

The previous example indicates how random matrix theory results are more generally used in practice [37]. Namely, different limiting quantities (Eigenvalue Density Function, Stieltjes transforms, moments) are evaluated in the limit when $m, n \rightarrow \infty$. However, they converge rapidly with increasing $m$ and $n$ and a good agreement between the analytical expressions and the experimentally obtained counterparts is achieved even for relatively small $m$ and $n$. Therefore the expectations of these quantities for finite $m$ and $n$ are approximated with the corresponding asymptotic characterizations parameterized with finite $c=m / n$.

Formally, provided that the corresponding limiting Eigenvalue Density Function exists, the expected values of the eigenvalue and eigenvector Stieltjes transforms for a $m$-by- $m$ random and Hermitian matrix $\mathbf{A}_{m}$ are approximated by

$$
\begin{aligned}
& \mathbf{E}\left[S_{\mathbf{A}_{m}}(z ; m, n)\right] \approx \bar{S}_{\mathbf{A}}\left(z ; c=\frac{m}{n}\right) \\
& \mathbf{E}\left[F_{\mathbf{A}_{m}}(z ; m, n)\right] \approx \bar{F}_{\mathbf{A}}\left(z ; c=\frac{m}{n}\right)
\end{aligned}
$$

Similarly, the expectation of the $k$-th order moment for finite $m$ and $n$ is approximated 
with the limiting $k$-th order moment

$$
\mathbf{E}\left[M_{k}(m, n)\right] \approx \bar{M}_{k}\left(\frac{m}{n}\right) .
$$




\section{Chapter 3}

\section{Spatial Power Spectum Estimation}

\subsection{Introduction}

Spatial spectrum estimation consists of estimating the power received at an array of sensors as a function of direction of arrival. The power of the signal arriving from a particular direction can be estimated using the Minimum Power Distortionless Response (MPDR) beamformer [52]. The MPDR beamformer array weights depend on the spatial correlation matrix of the received signal as received at the array of sensors. Usually, the spatial correlation matrix is unknown and must be estimated from the received data. Due to the time-varying nature of the environment and the fact that an array can contain large number of sensors, the number of snapshots that can be collected over the approximate stationarity of the environment might be insufficient to accurately estimate the correlation matrix. ${ }^{1}$ Diagonal loading, also known as Tikhonov regularization, is extensively used to address this problem. This approach consists of adding a small regularization matrix, usually a scaled identity matrix, to the estimated spatial correlation matrix, with the goal of reducing the L2 norm of the resulting array weights and thus, the sensitivity of the beamformer to the model mismatch caused by the deficient sample support. A problem that arises is how to choose an optimal regularization such that a certain performance metric such

\footnotetext{
${ }^{1}$ Note also that the spatial coherence of the environment might be lost if the array has long aperture. This problem is not considered here.
} 
as mean square error (MSE) is optimized. This chapter considers such a problem.

The statistical characterization of the diagonally loaded MPDR beamformer output has received considerable attention in the literature. The mean value and variance of the MPDR beamformer output is studied in [11] by assuming a complex Gaussian received signal, zero diagonal loading in the computation of array weights and that the number of snapshots is larger than the number of sensors. The probability density function of the MPDR output when the received signal is complex Gaussian and at most two signals are present in the sensed field is studied in [37]. The work in [35] characterizes the expected value of the signal-to-interference-plus-noise ratio (SINR) at the MPDR beamformer output. A study of the probability density function of the SNR of the diagonally loaded MPDR beamformer output using the Gaussian method, introduced in Section 2.6, is reported in [44] and [45].

This chapter studies a diagonal loading problem for two commonly used spatial power spectrum estimators based on the MPDR beamformer [3]. The MSE between the estimated and true or spatial power spectra obtained with the ensemble correlation matrix, is adopted as a performance metric and one of the main goals is to explore how the optimal diagonal loading changes with steering direction in a snapshot deficient regime. In doing so, we study how the power estimators behave with respect to diagonal loading, number of snapshots and number of sensors. This is done in several steps.

First, we analyze behavior of the power estimators, their expectations, variances and MSE's in the limit when the number of snapshots and sensors grow large at the same rate. It is shown that both power estimators for a fixed diagonal loading and steering direction almost surely converge to non-random quantities in the limit when the numbers of snapshots and sensors grow large at the same rate. When the input process is Gaussian, the variances and MSE's of the power estimators are characterized in the limit, along with the rates of convergence.

Second, we study the interplay between the bias and variance in determining the optimal diagonal loading which minimizes the estimation MSE. We conjecture that the variance of the considered power estimators does not significantly impact the 
value of the optimal diagonal loading. Thus, the increase in MSE due to diagonal loading is primarily due to an increase in the squared bias and diagonal loading that is chosen to minimize the squared bias has an MSE that is close to that of the weights chosen to minimize the MSE.

Third, we investigate how optimal diagonal loading behaves with respect to steering direction when the arrival process consists of one or more plane waves contaminated with uncorrelated noise. It is shown that the optimal diagonal loading increases as the steering direction moves away from a point source and follows an oscillatory behavior.

Finally, the MSE performances of the two power estimators are compared and is shown that one of them performs better (lower MSE) at the expense of increased sensitivity to diagonal loading.

The rest of this chapter is organized as follows. A background on MPDR based power estimation, along with the problem formulation, is presented in Section 3.2. A suitable representation of the power estimators, two approaches in computing the true power, main assumptions and simulation scenarios used throughout the chapter are introduced in Section 3.3. The preliminary insights into behavior of power estimators when viewed as functions of diagonal loading are presented in Section 3.4. The asymptotic behavior of power estimators is derived in Section 3.5. The derivation of the asymptotic behavior of the variance and MSE corresponding to power estimators is presented in Section 3.6. Section 3.7 analyses how the squared bias and variance impact the value of optimal diagonal loading. Section 3.8 investigates the value of the optimal diagonal loading in scenarios in which a single or multiple sources are embedded in uncorrelated noise. The relative performances of the two power estimators are compared in Section 3.9. Finally, Section 3.10 concludes this chapter.

Throughout this section, $\left(\mathbf{s}^{H} \mathbf{A}\right)_{i}$ denotes an inner product between a vector $\mathbf{s}$ and the $i$ th column of a matrix A. Similarly, $(\mathbf{A s})_{j}$ is an inner product between the $j$ th row of $\mathbf{A}$ and $\mathbf{s}$. Also, $\|\mathbf{A}\|$ denotes a Frobenius norm and $x=O(\alpha)$ means that $x \leq K \alpha$, for some constant scalar $K$. 


\subsection{Background}

An array of $m$ sensors receives at discrete time $k$ a signal whose Fourier coefficients in a frequency bin of interest and across the array constitute an observation vector $\mathbf{u}(k) \in \mathbf{C}^{m}$, known as a snapshot. A snapshot contains signatures of the useful signal, interferers and noise. The ensemble correlation between snapshots received at times $i$ and $j$ is

$$
\mathbf{E}\left[\mathbf{u}(i) \mathbf{u}^{H}(j)\right]=\mathbf{R} \delta_{D}(i-j),
$$

where $\delta_{D}(x)$ is the Dirac delta function. The literature usually refers to the correlation matrix $\mathbf{R}$ as a temporal frequency spatial correlation matrix [52].

The MPDR beamformer passes a signal arriving from a look direction characterized by the replica vector $\mathbf{v}_{s}$ undistorted and minimizes the output power, i.e., [52]

$$
\mathbf{w}_{\mathrm{MPDR}}\left(\mathbf{v}_{s}\right)=\underset{\mathbf{w}}{\arg \min } \quad \mathbf{w}^{H} \mathbf{R} \mathbf{w} \quad \text { s.t. } \quad \mathbf{w}^{H} \mathbf{v}_{s}=1 .
$$

In a simple model of a plane wave arriving from elevation angle $\theta$ on a linear, uniform and vertical array with inter-element spacing $d$, the signal replica vector $\mathbf{v}_{s}$ is given by $[52]$

$$
\mathbf{V}_{s}(\theta)=\left[\begin{array}{llll}
1 & e^{-j \frac{2 \pi}{u} d \cos (\theta)} & \ldots & e^{-j \frac{2 \pi}{u}(m-1) d \cos (\theta)}
\end{array}\right]^{H},
$$

where $u$ is the signal wavelength. Here, $\theta=0$ indicates a signal propagating vertically in the downward direction and $\theta=\pi / 2$ indicates a signal propagating horizontally (i.e., broadside to the array). While the model in (3.3) is for a plane wave received at a linear uniform array, the results developed here are equally applicable to a general signal arising in the contexts of non-linear and non-uniform arrays as well as in matched field processing problems.

The solution to (3.2) is given in a closed form by [52]

$$
\mathbf{w}_{\mathrm{MPDR}}\left(\mathbf{v}_{s}\right)=\frac{\mathbf{R}^{-1} \mathbf{v}_{s}}{\mathbf{v}_{s}^{H} \mathbf{R}^{-1} \mathbf{v}_{s}} .
$$

The number of sources and their respective received powers can be estimated by 
steering the MPDR beamformer across all possible look directions. Hence, the power of the signal arriving from the direction corresponding to the replica vector $\mathbf{v}_{s}$ is

$$
P_{\mathrm{MPDR}}\left(\mathbf{v}_{s}\right)=\mathbf{w}_{\mathrm{MPDR}}^{H}\left(\mathbf{v}_{s}\right) \mathbf{R} \mathbf{w}_{\mathrm{MPDR}}\left(\mathbf{v}_{s}\right)=\frac{1}{\mathbf{v}_{s}^{H} \mathbf{R}^{-1} \mathbf{v}_{s}}
$$

The correlation matrix $\mathbf{R}$ is usually unknown and is estimated from the observed data. We consider in this chapter a sample correlation matrix (SCM) $\hat{\mathbf{R}}$ evaluated from $n$ rectangularly windowed observations defined as

$$
\hat{\mathbf{R}}=\frac{1}{n} \sum_{i=1}^{n} \mathbf{u}(i) \mathbf{u}^{H}(i)
$$

As elaborated in Section 1.2, the number of snapshots that can be collected in the interval over which the environment can be considered stationary, $n$, might be insufficient to accurately estimate $\mathbf{R}$, which leads to one type of model mismatch [52]. To combat the sensitivity to mismatch and/or to improve the condition number of $\hat{\mathbf{R}}$, a diagonally loaded SCM $\hat{\mathbf{R}}_{\delta}$ is introduced

$$
\hat{\mathbf{R}}_{\delta}=\hat{\mathbf{R}}+\delta \mathbf{I}
$$

where $\delta$ is a diagonal loading parameter.

The MPDR array weight vector evaluated with a diagonally loaded SCM is the solution to the following problem

$$
\mathbf{w}_{\mathrm{MPDR}}\left(\mathbf{v}_{s}\right)=\arg \min _{\mathbf{w}} \mathbf{w}^{H} \hat{\mathbf{R}} \mathbf{w}+\delta \mathbf{w}^{H} \mathbf{w} \text { s.t. } \mathbf{w}^{H} \mathbf{v}_{s}=1 .
$$

Thus, array weight vectors with large L2 norms are penalized and the L2 norm of the resulting array weight vector decreases monotonically with increasing $\delta$. Since the effect of insufficient sample support is a type of modeling mismatch in the SCM [52] and the sensitivity of the performance of an array weight vector on model mismatch is proportional to its L2 norm squared, computing the array weights with a diagonally loaded SCM will reduce the negative impact of insufficient sample support on 
processor performance. However, reducing the L2 norm of the array weights will also reduce its ability to resolve closely spaced sources or attenuate nearby interfering signals while passing the signal from the steering direction undistorted. The choice of the optimal diagonal loading balances these two factors and, as we show, depends on both the sample support of the SCM and how far the steering direction is from nearby interfering signals.

The power of the signal arriving from direction $\mathbf{v}_{s}$ is estimated from (3.5) where the MPDR weights are evaluated using (3.4) with a diagonally loaded SCM $\hat{\mathbf{R}}_{\delta}$ and the SCM $\hat{\mathbf{R}}$ is used instead of $\mathbf{R}$ in (3.5). That is [3],

$$
\hat{P}_{\mathrm{a}}\left(\delta, \mathbf{v}_{s}\right)=\frac{\mathbf{v}_{s}^{H} \hat{\mathbf{R}}_{\delta}^{-1} \hat{\mathbf{R}} \hat{\mathbf{R}}_{\delta}^{-1} \mathbf{v}_{s}}{\left[\mathbf{v}_{s}^{H} \hat{\mathbf{R}}_{\delta}^{-1} \mathbf{v}_{s}\right]^{2}}
$$

If diagonally loaded SCM $\hat{\mathbf{R}}_{\delta}$ is substituted in (3.5) instead of $\mathbf{R}$, an alternative and more compact form is obtained [3]

$$
\hat{P}_{\mathrm{b}}\left(\delta, \mathbf{v}_{s}\right)=\frac{1}{\mathbf{v}_{s}^{H} \hat{\mathbf{R}}_{\delta}^{-1} \mathbf{v}_{s}} .
$$

Broadly speaking, this chapter studies how diagonal loading impacts the performance of these two adaptive beamformers in the snapshot deficient regime.

The performance of the power estimators (3.8) and (3.9) is measured via estimation mean square error (MSE). Given a steering direction $\mathbf{v}_{s}$, the MSE of the power estimator $\hat{P}$ is viewed as a function of the loading $\delta$ and represented via the bias-variance decomposition as

$$
\operatorname{MSE}(\delta)=\mathbf{E}^{2}[\hat{P}(\delta)-P]+\operatorname{var}(\hat{P}(\delta))
$$

where the first term is a squared bias, which we denote by $\operatorname{bias}^{2}(\delta)$, while $P$ is the true power of the signal arriving from the considered direction $\mathbf{v}_{s}$. In the absence of a subscript, $\hat{P}$ refers to either of the two estimators. 
The optimal diagonal loading $\delta_{\text {opt }}$ is defined as

$$
\delta_{\mathrm{opt}}=\arg \min _{\delta} \operatorname{MSE}(\delta)
$$

In addition, a diagonal loading which minimizes the squared bias is denoted by $\tilde{\delta}_{\text {opt }}$ and formally defined as

$$
\tilde{\delta}_{\text {opt }}=\arg \min _{\delta} \operatorname{bias}^{2}(\delta)
$$

This chapter develops an understanding of how the MSE performance in a snapshot deficient regime depends on diagonal loading. In achieving this, we study how a diagonal loading $\delta$, number of sensors $m$ and number of snapshots $n$ impact the squared bias and variance of the power estimators.

\subsection{Preview and Assumptions}

This section introduces an alternative expression for power estimators, presents a particular model for the snapshots and summarizes the assumptions used in the theoretical analysis. Also, the true power of a signal impinging upon an array is defined using two different approaches. Finally, two scenarios used in the simulations that validate the insights and derived characterizations in this chapter are presented.

\subsubsection{Alternative Expressions for Power Estimators}

The power estimators $\hat{P}_{a}$ and $\hat{P}_{b}$ are represented via quadratic forms $Q_{k}$, defined as

$$
Q_{k}(\delta)=\mathbf{v}_{s}^{H} \hat{\mathbf{R}}_{\delta}^{-k} \mathbf{v}_{s}
$$

and viewed as functions of diagonal loading $\delta$ for a fixed steering direction $\mathbf{v}_{s}$. Therefore, the power estimator $\hat{P}_{b}$ is expressed as

$$
\hat{P}_{b}\left(\delta, \mathbf{v}_{s}\right)=\frac{1}{Q_{1}(\delta)}
$$


On the other hand, solving (3.6) for $\hat{\mathbf{R}}$ and substituting it into (3.8) yields

$$
\begin{aligned}
\hat{P}_{a}\left(\delta, \mathbf{v}_{s}\right) & =\frac{\mathbf{v}_{s}^{H} \hat{\mathbf{R}}_{\delta}^{-1}\left(\hat{\mathbf{R}}_{\delta}-\delta \mathbf{I}\right) \hat{\mathbf{R}}_{\delta}^{-1} \mathbf{v}_{s}}{\left[\mathbf{v}_{s}^{H} \hat{\mathbf{R}}_{\delta}^{-1} \mathbf{v}_{s}\right]^{2}} \\
& =\frac{1}{Q_{1}(\delta)}-\delta \frac{Q_{2}(\delta)}{Q_{1}^{2}(\delta)}
\end{aligned}
$$

The analysis throughput this chapter uses these two alternative expressions for the power estimators.

\subsubsection{Gaussian Snapshot Model}

Assuming the ensemble correlation matrix of the snapshots is $\mathbf{R}$, the rectangularly windowed SCM (3.5) is modeled as described in Section 2.5.2 as

$$
\hat{\mathbf{R}}=\frac{1}{n} \mathbf{R}^{\frac{1}{2}} \mathbf{X X \mathbf { R } ^ { \frac { 1 } { 2 } }}
$$

where $\mathbf{X}$ is a complex $m$-by- $n$ matrix with i.i.d. entries of zero mean and unit variance. $\mathbf{R}^{\frac{1}{2}}$ is the Hermitian positive definite square root of the correlation matrix $\mathbf{R}$.

In the asymptotic study of the variances associated with power estimators, we assume the input snapshots are Gaussian distributed. ${ }^{2}$ This assumption allows us to represent the SCM in a more convenient form.

The eigenvalues of the correlation matrix $\mathbf{R}$ are denoted by $\lambda_{1}, \lambda_{2}, \ldots, \lambda_{m}$, and collected into a diagonal eigenvalue matrix $\mathbf{D}$, such that the eigen-decomposition of $\mathbf{R}$ is given by $\mathbf{R}=\mathbf{Q D Q}^{H}$. Using (3.16), a diagonally loaded SCM (3.6) is expressed as

$$
\hat{\mathbf{R}}_{\delta}=\frac{1}{n} \mathbf{Q D}^{\frac{1}{2}} \mathbf{Q}^{H} \mathbf{X} \mathbf{X}^{H} \mathbf{Q D}^{\frac{1}{2}} \mathbf{Q}^{H}+\delta \mathbf{I} .
$$

Since the received snapshots are Gaussian, matrix $\mathbf{X}$ is unitary invariant. Then, since $\mathbf{Q}$ is a unitary matrix, $p\left(\mathbf{Q}^{H} \mathbf{X}\right)=p(\mathbf{Q})$ where $p(\mathbf{B})$ denotes a joint probability distribution of the elements of a matrix B. Therefore, statistically, a matrix $\mathbf{Q}^{H} \mathbf{X}$

\footnotetext{
${ }^{2}$ Note that the corresponding SCM $\hat{\mathbf{R}}$ has Wishart distribution.
} 
in (3.17) can be replaced by $\mathbf{X}$. Hence, introducing

$$
\mathbf{Y}=\mathbf{D}^{\frac{1}{2}} \mathbf{X},
$$

a diagonally loaded SCM $\hat{\mathbf{R}}_{\delta}$ is in a statistical sense equivalently represented as

$$
\begin{aligned}
\hat{\mathbf{R}}_{\delta} & \doteq \frac{1}{n} \mathbf{Q D}^{\frac{1}{2}} \mathbf{X} \mathbf{X}^{H} \mathbf{D}^{\frac{1}{2}} \mathbf{Q}^{H}+\delta \mathbf{I} \\
& =\frac{1}{n} \mathbf{Q} \mathbf{Y} \mathbf{Y}^{H} \mathbf{Q}^{H}+\delta \mathbf{I} \\
& =\mathbf{Q}\left(\frac{1}{n} \mathbf{Y} \mathbf{Y}^{H}+\delta \mathbf{I}\right) \mathbf{Q}^{H}
\end{aligned}
$$

Note that the right hand sides of (3.17) and (3.19) are not equal, but have the same joint probability distribution of the elements. Consequently, the moments of their functionals are the same.

Using (3.19) and definition (3.13), a quadratic form $Q_{k}$ is expressed as

$$
\begin{aligned}
Q_{k} & =\mathbf{v}_{s}^{H} \mathbf{Q}\left(\frac{1}{n} \mathbf{Y} \mathbf{Y}^{H}+\delta \mathbf{I}\right)^{-1} \mathbf{Q}^{H} \mathbf{v}_{s} \\
& =t^{k} \mathbf{s}^{H} \mathbf{H}^{k}(t) \mathbf{s},
\end{aligned}
$$

where $\mathbf{s}=\mathbf{Q}^{H} \mathbf{v}_{s}, t=\frac{1}{\delta}$ and

$$
\mathbf{H}(t)=\left(\mathbf{I}+\frac{t}{n} \mathbf{Y} \mathbf{Y}^{H}\right)^{-1}
$$

is a resolvent matrix.

Note that the quadratic forms $Q_{k}$ are functionals of Gaussian matrix $\mathbf{Y}$ (3.18) which satisfies the conditions of integration by parts formula (2.66) and PoincareNash inequality (2.67). Thus, under the assumption that the received snapshots are Gaussian distributed, we evaluate the variances of the power estimators using the Gaussian method. 


\subsubsection{Assumptions}

The main assumptions used throughout this chapter are listed here. Namely,

1. The number of snapshots $n$ and number of sensors $m$ are of the same order. If not specified otherwise, this means that

$$
0<\lim \inf \frac{m}{n} \leq \lim \sup \frac{m}{n}<\infty
$$

Note that $m / n$ does not necessarily need to converge. All what is required is the ratio be non-zero and finite. Note that this condition also implies that $m$ and $n$ scale linearly in the limit.

2. The eigenvalues of the ensemble correlation matrix $\mathbf{R}$ are uniformly upper bounded for all $m$, i.e.,

$$
\max \left\{\lambda_{1}, \lambda_{2}, \ldots, \lambda_{m}\right\} \leq D_{m}<\infty
$$

3. The eigenvalues of the SCM $\hat{\mathbf{R}}$, denoted by $\hat{\lambda}_{1}, \ldots, \hat{\lambda}_{m}$ are uniformly upper bounded for all $m$, i.e.,

$$
\max \left\{\hat{\lambda}_{1}, \ldots, \hat{\lambda}_{m}\right\} \leq \hat{D}_{m}<\infty
$$

Note that these eigenvalues are lower bounded by some $\hat{d}_{m} \geq 0$.

4. The norm of the signal replica vector, $\mathbf{v}_{s}$, is uniformly upper bounded for all $m$ such that

$$
\left\|\mathbf{v}_{s}\right\|=\|\mathbf{s}\| \leq S_{m}=O(\sqrt{m})
$$

Note that for any array in a plane wave environment where the magnitude of each element of the replica vector equals $1,\left\|\mathbf{v}_{s}\right\|=\sqrt{m}$. 


\subsubsection{Definitions of True Power}

The estimation MSE performance of power estimators characterizes how "close" an estimate is to the true value of the power. While there is no ambiguity what true power is, we introduce here an alternative definition which is in some sense better suited for the study of estimation performance in snapshot deficient regime.

\section{Traditional Definition of True Power}

For completeness, the traditional ${ }^{3}$ way of defining the true power corresponding to a specific arrival model is presented in this part.

Namely, if the received signal is composed of a number of signals, each having power $P_{i}$ and impinging on the array in the direction described by a replica vector $\mathbf{v}_{i}$, the true power in the steering direction $\mathbf{v}_{s}$ is defined as [26]

$$
P\left(\mathbf{v}_{s}\right)=\left\{\begin{array}{c}
\frac{\sigma_{v}^{2}\left(\mathbf{v}_{s}\right)}{m}, \text { if } \mathbf{v}_{s} \neq \mathbf{v}_{i} \\
P_{i}+\frac{\sigma_{v}^{2}\left(\mathbf{v}_{s}\right)}{m}, \text { if } \mathbf{v}_{s}=\mathbf{v}_{i}
\end{array}\right.
$$

where $\sigma_{v}^{2}\left(\mathbf{v}_{s}\right)$ is the level of the noise power spectral density in the direction described by $\mathbf{v}_{s}$. We assume the noise is uncorrelated and the inter-element spacing is half-awavelength such that $\sigma_{v}^{2}\left(\mathbf{v}_{s}\right)=\sigma_{v}^{2}$.

\section{Alternative Definition of True Power}

Alternatively, true power can be defined as the power that would be estimated if the ensemble correlation matrix $\mathbf{R}$ was known. ${ }^{4}$ Since no loading is needed in this case, this power is given by

$$
P=\frac{1}{\mathbf{v}_{s}^{H} \mathbf{R}^{-1} \mathbf{v}_{s}} .
$$

The justification for this definition is found in the fact that even when the correlation matrix $\mathbf{R}$ is known, the estimators $\hat{P}_{a}$ and $\hat{P}_{b}$ are unable to accurately estimate the

\footnotetext{
${ }^{3}$ Traditional, in the sense that it is usually used for the power density spectrum estimation of time series data.

${ }^{4}$ In fact, this is a more useful definition in the context of spectrum estimation using an array of sensors.
} 
power when steering very close to the source direction. When the SCM $\hat{\mathbf{R}}$ is estimated from a limited number of snapshots, the best that one can do is to approach the performance achieved with a known correlation matrix $\mathbf{R}$.

\subsubsection{Simulation Scenarios}

The theoretical results developed in this chapter are validated via Monte-Carlo simulations. A standard, vertical, linear array with $u / 2$ (half-a-wavelength) separation of the elements is considered in the simulations. In addition, a spatially uncorrelated, zero-mean noise with a variance of one corrupts the signal snapshots. The following two different arrival structures are considered.

- In Scenario 1, 2 signals are arriving at elevation angles of $90^{\circ}$ and $92^{\circ}$ with respect to the broadside of the array and each has power 10 (i.e., the SNR is $10 \mathrm{~dB})$. The array contains 30 sensors and 50 snapshots are used to estimate the SCM. Note that the ratio between the array aperture and wavelength is 15 . Also, note that $c=0.6$ and the normalized trace of the ensemble correlation matrix of the input process is 21 .

- In Scenario 2, 2 signals are arriving at elevation angles of $90^{\circ}$ and $94^{\circ}$ with respect to the broadside of the array. Their SNR's are respectively $1 \mathrm{~dB}$ and 5 dB. The array has 40 sensors and the number of snapshots available to estimate the SCM is 25 . Note that the ratio between the array aperture and wavelength is 20. Also note that $c=1.6$ and the normalized trace of the ensemble correlation matrix is 5.42 .

\subsection{Preliminary Results on Behavior of Power Es- timators}

The power estimators $\hat{P}_{\mathrm{a}}$ and $\hat{P}_{\mathrm{b}}$, viewed as functions of diagonal loading $\delta$, are studied in this section. In particular, some results concerning the behavior of squared bias and 
variance with respect to diagonal loading $\delta$ are presented. Throughout the section, we do not explicitly show the dependence on steering direction $\mathbf{v}_{s}$ in the notation for power estimators, i.e., with slight abuse of notation we write $\hat{P}_{a}(\delta)$ and $\hat{P}_{b}(\delta)$.

\subsubsection{Dependence of Squared Bias on Diagonal Loading}

A study of how the squared bias, $\operatorname{bias}^{2}(\delta)$, depends on $\delta$ is centered around exploring how the estimators and their expectations depend on $\delta$. The results concerning the behavior of the estimators $\hat{P}_{a}$ and $\hat{P}_{b}$ are summarized in the following lemma.

Lemma 3.1. For any (Hermitian non-negative definite) $S C M \hat{\mathbf{R}}$ and for $\delta \geq 0$, under assumption 3., the following holds

1. $\hat{P}_{b}(\delta) \geq \hat{P}_{a}(\delta)$, with equality if and only if $\delta=0$.

2. $\hat{P}_{a}(\delta)$ is monotonically increasing for $0<\delta<\infty$, unless all the eigenvalues of $\hat{\mathbf{R}}$ are equal. Its slope is zero at $\delta=0^{+}$(i.e., when $\delta$ approaches 0 from the positive side) and when $\delta \rightarrow \infty$.

3. $\hat{P}_{b}(\delta)$ is strictly monotonically increasing for all $0 \leq \delta \leq \infty$.

Proof. 1. From (3.14) and (3.15), two power estimators are related as

$$
\hat{P}_{a}(\delta)=\hat{P}_{b}(\delta)-\delta \frac{Q_{2}}{Q_{1}^{2}}
$$

Since the sample eigenvalues are uniformly upper bounded, $Q_{2}>0$. Therefore, $\hat{P}_{b} \geq \hat{P}_{a}$. The equality holds when $\delta=0$.

2. Denoting the eigenvalues and eigenvectors of $\hat{\mathbf{R}}$ with $\hat{\lambda}_{i}$ and $\hat{\mathbf{q}}_{i}$, the power estimator $\hat{P}_{a}$ is using (3.8) expressed as

$$
\hat{P}_{a}=\sum_{i=1}^{m} \frac{b_{i} \hat{\lambda}_{i}}{\left(\hat{\lambda}_{i}+\delta\right)^{2}}\left(\sum_{i=1}^{m} \frac{b_{i}}{\hat{\lambda}_{i}+\delta}\right)^{-2}
$$


where $b_{i}=\left|\mathbf{v}_{s}^{H} \hat{\mathbf{q}}_{i}\right|^{2}$. Taking the first derivative of $\hat{P}_{a}$ with respect to $\delta$ yields

$$
\left.\frac{d \hat{P}_{a}}{d \delta}=2 \delta \sum_{i, j: i<j} \frac{b_{i} b_{j}\left(\hat{\lambda}_{i}-\hat{\lambda}_{j}\right)^{2}}{\left(\hat{\lambda}_{i}+\delta\right)^{3}\left(\hat{\lambda}_{j}+\delta\right)^{3}} \frac{b_{i}}{\hat{\lambda}_{i}+\delta}\right)^{-2}
$$

Therefore, unless all the eigenvalues of $\hat{\mathbf{R}}$ are equal, $\frac{d \hat{P}_{a}}{d \delta}$ is positive for finite, non-zero loading $\delta$. On the other hand, the slope of $\hat{P}_{a}$ is zero for $\delta=0$ and when $\delta \rightarrow \infty$. Consequently, $\hat{P}_{a}$ is convex when $\delta \rightarrow 0^{+}$and concave when $\delta \rightarrow \infty$. Furthermore, $\hat{P}_{a}$ converges to a finite value both when $\delta \rightarrow \infty$ and when $\delta \rightarrow 0^{+}$.

3. The estimator $\hat{P}_{b}$ can be expressed in terms of $b_{i}$ and $\hat{\lambda}_{i}$ as

$$
\hat{P}_{b}=\left(\sum_{i=1}^{m} \frac{b_{i}}{\lambda_{i}+\delta}\right)^{-1}
$$

The first derivative of $\hat{P}_{b}$ with respect to loading $\delta$ is then given by

$$
\frac{d \hat{P}_{b}}{d \delta}=\sum_{i} \frac{b_{i}}{\left(\hat{\lambda}_{i}+\delta\right)^{2}}\left(\sum_{i} \frac{b_{i}}{\hat{\lambda}_{i}+\delta}\right)^{-2}
$$

Since $\frac{d \hat{P}_{b}}{d \delta}>0$, the estimator $\hat{P}_{b}$ is a strictly increasing function. Its slope is positive at $\delta=0$. When $\delta \rightarrow \infty$, its slope converges to $1 / m$.

In words, both estimators $\hat{P}_{a}$ and $\hat{P}_{b}$ are monotonically non-decreasing functions of $\delta$. While $\hat{P}_{a}$ converges to a finite value as $\delta \rightarrow \infty$, the estimator $\hat{P}_{b}$ is unbounded. For the same non-zero loading $\delta$, the estimator $\hat{P}_{b}$ is greater than $\hat{P}_{a}$.

These results carry over to $\mathbf{E}\left[\hat{P}_{a}(\delta)\right]$ and $\mathbf{E}\left[\hat{P}_{b}(\delta)\right]$ whenever the derivative and expectation can interchange the order. The following lemma summarizes how the expected values of the power estimators behave when $\delta=0$ and $\delta \rightarrow \infty$. The result corresponding to the case of $\delta=0$ follows from later development, but is presented here for completeness. 
Lemma 3.2. Assuming the expectation is taken over all possible realizations of the $S C M \hat{\mathbf{R}}$ and $\delta \geq 0$,

1. For any fixed $m$ and $n, \lim _{\delta \rightarrow \infty} \mathbf{E}\left[\hat{P}_{a}(\delta)\right]=\frac{1}{m^{2}} \mathbf{v}_{s}^{H} \mathbf{R} \mathbf{v}_{s}$.

2. In the limit when $m, n \rightarrow \infty$ at the same rate such that $\frac{m}{n} \rightarrow c \in(0,1)$, for both estimators, $\hat{P}(0) \rightarrow \frac{1-c}{\mathbf{v}_{s}^{H} \mathbf{R}^{-1} \mathbf{v}_{s}}$, almost surely.

Proof. 1. When $\delta \rightarrow \infty$, the MPDR beamformer becomes a matched filter (i.e., the conventional beamformer) with array weight vector $\mathbf{w}\left(\mathbf{v}_{s}\right)=\frac{\mathbf{v}_{s}}{\mathbf{v}_{s}^{H} \mathbf{v}_{s}}$. Therefore,

$$
\lim _{\delta \rightarrow \infty} \hat{P}_{a}(\delta)=\frac{1}{m^{2}} \mathbf{v}_{s}^{H} \hat{\mathbf{R}} \mathbf{v}_{s}
$$

The proof is completed after taking the expectation of both sides of (3.30).

2. This result follows directly from Lemma 3.4 by taking $\delta=0$. Recall that due to rapid convergence,

$$
\mathbf{E}[\hat{P}(0)] \approx \frac{1-m / n}{\mathbf{v}_{s}^{H} \mathbf{R}^{-1} \mathbf{v}_{s}} .
$$

Note that if $m>n$ and $\delta=0$, both power estimates are equal to 0 , unless some sort of subspace processing is employed.

The functional dependence of $\mathbf{E}\left[\hat{P}_{a}\right]$ and $\mathbf{E}\left[\hat{P}_{b}\right]$ on diagonal loading $\delta$ is visualized in Fig. 3-1. Although the plot is generated for a specific scenario, this behavior is typical.

So far we have considered how power estimators and their expectations depend on diagonal loading. We now examine how the squared bias associated with the power estimators depends on diagonal loading. Namely, the behavior of the squared bias is directly implied from the preceding results and summarized as follows. As such, for a non-negative diagonal loading $\delta$,

1. The squared bias corresponding to the power estimator $\hat{P}_{a}$ attains a unique global minimum, equal to zero, at a finite, non-zero $\tilde{\delta}_{\text {opt }}$ if the true power $P$ satis- 

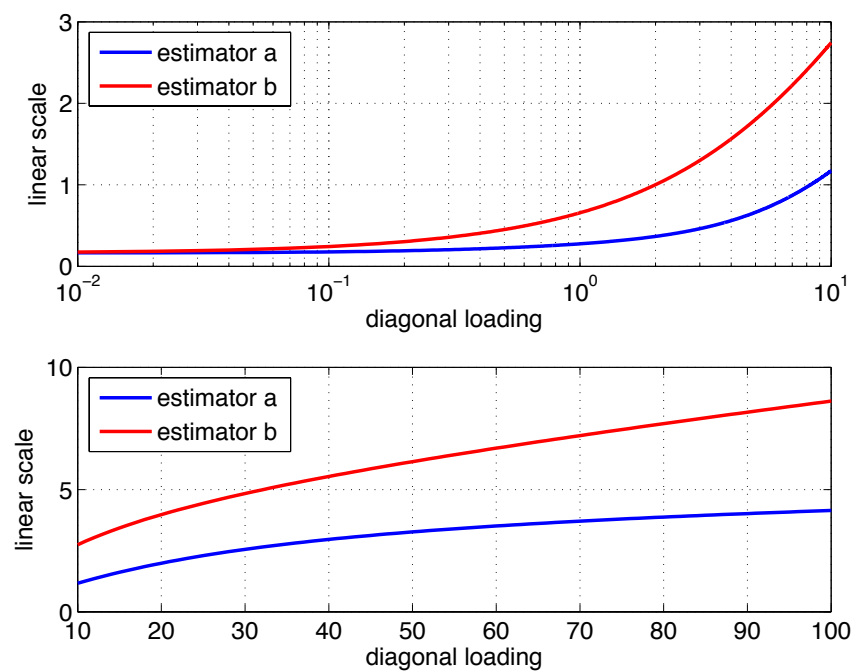

Figure 3-1: Typical behavior of the expectations of the estimators $\hat{P}_{a}$ and $\hat{P}_{b}$. The scenario used to generate the plots is described in the caption of Fig. 3-28. The steering angle is $89^{\circ}$. The normalized trace of the ensemble correlation matrix of the considered model is 21 .

fies $\mathbf{E}\left[\hat{P}_{a}(0)\right]<P<\lim _{\delta \rightarrow \infty} \mathbf{E}\left[\hat{P}_{a}(\delta)\right]$. If the true power $P \geq \lim _{\delta \rightarrow \infty} \mathbf{E}\left[\hat{P}_{a}(\delta)\right]$, then the squared bias corresponding to $\hat{P}_{a}$ decays as $\delta \rightarrow \infty$.

2. The squared bias corresponding to the power estimator $\hat{P}_{b}$ attains a unique global minimum, equal to zero, at a finite, non-zero $\tilde{\delta}_{\text {opt }}$ if the true power $P$ satisfies $\mathbf{E}\left[\hat{P}_{b}(0)\right]<P<\infty$.

3. If the true power $P \leq \mathbf{E}[\hat{P}(0)]$, then the squared bias of either of the estimators is minimized at $\tilde{\delta}_{\text {opt }}=0$.

4. The squared bias is monotonically decreasing for $0 \leq \delta \leq \tilde{\delta}_{\text {opt }}$ and monotonically increasing for $\delta \geq \tilde{\delta}_{\text {opt }}$.

\subsubsection{Dependence of Variance on Diagonal Loading}

This part presents results which show how the variances of the power estimators $\hat{P}_{a}$ and $\hat{P}_{b}$ behave with respect to loading $\delta$.

First, under assumption 2, the norm of the SCM $\hat{\mathbf{R}}$ is uniformly bounded and consequently $\hat{P}_{a}, \hat{P}_{a}^{2}, \hat{P}_{b}$ and $\hat{P}_{b}^{2}$ are all uniformly bounded. This implies that the first 
and second moments of the estimators are also bounded. Therefore, the variances of the power estimators are finite for a finite loading $\delta$.

Note that as $\delta \rightarrow \infty, \hat{P}_{a}$ converges to the power estimator associated with the matched filter, whose variance is finite. Consequently, the variance of $\hat{P}_{a}(\delta)$ is finite as $\delta \rightarrow \infty$.

In general, $\operatorname{var}\left(\hat{P}_{a}(\delta)\right) \neq \operatorname{var}\left(\hat{P}_{b}(\delta)\right)$. However, it turns out that these variances are equal when $\delta \rightarrow \infty$. From that result, we conclude that the variance of $\hat{P}_{b}$ is finite in the limit $\delta \rightarrow \infty$. In addition, if the input process is Gaussian, we are able to analytically characterize this variance. The results are summarized and proved in the following lemma.

Lemma 3.3. Under assumption 2, the following holds,

1. $\lim _{\delta \rightarrow \infty} \operatorname{var}\left(\hat{P}_{a}(\delta)\right)=\lim _{\delta \rightarrow \infty} \operatorname{var}\left(\hat{P}_{b}(\delta)\right)$

2. If the received snapshots $\mathbf{z}(i)$ are Gaussian distributed, then

$$
\lim _{\delta \rightarrow \infty} \operatorname{var}\left(\hat{P}_{a}(\delta)\right)=\frac{\left(\mathbf{v}_{s}^{H} \mathbf{R} \mathbf{v}_{s}\right)^{2}}{n m^{4}}
$$

Proof. 1. The difference between the two variances is after simple algebraic manipulations expressed as

$$
\operatorname{var}\left(\hat{P}_{a}\right)-\operatorname{var}\left(\hat{P}_{b}\right)=\mathbf{E}[A B]-\mathbf{E}[A] \mathbf{E}[B]
$$

where $A=\frac{\delta Q_{2}}{Q_{1}^{2}}$ and $B=\frac{\delta Q_{2}-2 Q_{1}}{Q_{1}^{2}}$. The goal is to find the limit of (3.33) when $\delta \rightarrow \infty$. This is solved by considering the leading order terms in $A$ and $B$. To do so, the quadratic form $Q_{k}, k \in\{1,2\}$ is expressed as

$$
\begin{aligned}
Q_{k} & =t^{k} \mathbf{v}_{s}^{H}(t \hat{\mathbf{R}}+\mathbf{I})^{-k} \mathbf{v}_{s} \\
& =t^{k} \sum_{i=1}^{m} \frac{\left|\mathbf{v}_{s}^{H} \mathbf{q}_{i}\right|^{2}}{\left(t \hat{\lambda}_{i}+1\right)^{k}}
\end{aligned}
$$

where $t=1 / \delta$. In the $t \rightarrow 0$ regime and since the eigenvalues $\hat{\lambda}_{i}$ are upper 
bounded, $t \hat{\lambda}_{i} \ll 1$. Therefore, using the Taylor series expansion of $\left(1+t \hat{\lambda}_{i}\right)^{-k}$ yields

$$
\begin{aligned}
& Q_{1}=t \sum_{j=0}^{\infty}(-1)^{j} t^{j} a_{j} \\
& Q_{2}=t^{2} \sum_{j=0}^{\infty}(-1)^{j}(j+1) t^{j} a_{j},
\end{aligned}
$$

where $a_{j}=\mathbf{v}_{s}^{H} \hat{\mathbf{R}}^{j} \mathbf{v}_{s}$. Substituting $Q_{1}$ and $Q_{2}$ into expressions for $A$ and $B$ yields

$$
\begin{aligned}
& A=\frac{1}{m t}-\frac{2 a_{1}}{m^{2}}+\frac{3 a_{2} t}{m^{2}}+O\left(t^{2}\right) \\
& B=-\frac{1}{m t}+\frac{a_{2} t}{m^{2}}+O\left(t^{2}\right) .
\end{aligned}
$$

Simple algebra further shows that $\mathbf{E}[A B]-\mathbf{E}[A] \mathbf{E}[B]=O(t)$, and thus

$$
\lim _{\delta \rightarrow \infty} \operatorname{var}\left(\hat{P}_{a}\right)-\operatorname{var}\left(\hat{P}_{b}\right)=0
$$

Since $\lim _{\delta \rightarrow \infty} \operatorname{var}\left(\hat{P}_{a}\right)$ exists, the two variances become equal when $\delta \rightarrow \infty$.

2. Pushing the limit operator inside the expectations and using (3.30) yields

$$
\begin{aligned}
& \lim _{\delta \rightarrow \infty} \operatorname{var}\left(\hat{P}_{a}(\delta)\right)=\operatorname{var}\left(\frac{1}{m^{2}} \mathbf{v}_{s}^{H} \hat{\mathbf{R}} \mathbf{v}_{s}\right) \\
& =\frac{1}{m^{4}}\left[\mathbf{E}\left[\left(\mathbf{v}_{s}^{H} \hat{\mathbf{R}} \mathbf{v}_{s}\right)^{2}\right]-\mathbf{E}^{2}\left[\mathbf{v}_{s}^{H} \hat{\mathbf{R}} \mathbf{v}_{s}\right]\right] .
\end{aligned}
$$

Substituting $\hat{\mathbf{R}}_{\delta}$ from (3.19) with $\delta=0$ into (3.40) yields

$$
\lim _{\delta \rightarrow \infty} \operatorname{var}\left(\hat{P}_{a}(\delta)\right)=\frac{1}{n^{2} m^{4}}\left[\mathbf{E}\left[\left(\mathbf{s}^{H} \mathbf{Y} \mathbf{Y}^{H} \mathbf{s}\right)^{2}\right]-\mathbf{E}^{2}\left[\mathbf{s}^{H} \mathbf{Y} \mathbf{Y}^{H} \mathbf{s}\right]\right]
$$

where $\mathbf{s}=\mathbf{v}_{s}^{H} \mathbf{Q}$. The first expectation in (3.41) is evaluated by unfolding the 
squared quadratic form and applying the integration by parts formula (2.66),

$$
\begin{aligned}
& \mathbf{E}\left[\left(\mathbf{s}^{H} \mathbf{Y} \mathbf{Y}^{H} \mathbf{s}\right)^{2}\right]=\sum_{i, j, k, p, q, r} s_{i}^{*} s_{k} s_{p}^{*} s_{r} \mathbf{E}\left[Y_{i j} Y_{k j}^{*} Y_{p q} Y_{r q}^{*}\right] \\
& \stackrel{(a)}{=} \sum_{i, j, k, p, q, r} s_{i}^{*} s_{k} s_{p}^{*} s_{r} \lambda_{i} \mathbf{E}\left[\frac{\partial\left(Y_{k j}^{*} Y_{p q} Y_{r q}^{*}\right)}{\partial Y_{i j}^{*}}\right] \\
& =\sum_{i, j, k, p, q, r} s_{i}^{*} s_{k} s_{p}^{*} s_{r} \lambda_{i} \mathbf{E}\left[\delta_{k i} Y_{p q} Y_{r q}^{*}+Y_{k j}^{*} Y_{p q} \delta_{i r} \delta_{j q}\right] \\
& \stackrel{(b)}{=} \sum_{i, j, k, p, q, r} s_{i}^{*} s_{k} s_{p}^{*} s_{r} \lambda_{i}\left(\lambda_{p} \delta_{k i} \delta_{p r}+\lambda_{p} \delta_{k p} \delta_{j q} \delta_{i r} \delta_{j q}\right) \\
& =\left(\mathbf{s}^{H} \mathbf{D s}\right)^{2}\left(n^{2}+n\right),
\end{aligned}
$$

where $\stackrel{(a)}{=}$ is obtained by applying $(2.66)$ with $f(\mathbf{Y})=Y_{k j}^{*} Y_{p q} Y_{r q}^{*}$, while $\stackrel{(b)}{=}$ is

obtained in a similar way with $f(\mathbf{Y})$ being $Y_{r q}^{*}$ for the first term and $Y_{k j}^{*}$ for the second term in the summand. Substituting (3.42) into (3.41) and noting that $\mathbf{E}\left[\mathbf{s}^{H} \mathbf{Y} \mathbf{Y}^{H} \mathbf{s}\right]=n \mathbf{s}^{H} \mathbf{D s}$ and $\mathbf{s}^{H} \mathbf{D s}=\mathbf{v}_{s} \mathbf{R} \mathbf{v}_{s}$, the proof is completed.

Note that from the last part of the preceding lemma, we conclude that for Gaussian input process, if the number of senors and observations are of the same order, as formalized in assumption 1., the variance of both estimators is $O\left(\mathrm{~m}^{-3}\right)$ (recall that under assumption $\left.4,\left\|\mathbf{v}_{s}\right\|_{2}=O(m)\right)$.

\subsection{Asymptotic Behavior of Power Estimators}

This section characterizes the asymptotic behavior of power estimators $\hat{P}_{a}$ and $\hat{P}_{b}$ for a fixed diagonal loading $\delta$. The theoretical result characterizing how power estimators and their expectations behave in the limit when $m, n \rightarrow \infty$ such that $m / n \rightarrow c \in$ $(0, \infty)$ are presented in the first part. Then, an asymptotically unbiased spatial power estimator is proposed. Finally, in the last part, the obtained results are validated via simulations. Throughout the section, we do not explicitly show the dependence on diagonal loading $\delta$ in the notation for power estimators, i.e., with slight abuse of 
notation we write $\hat{P}_{a}\left(\mathbf{v}_{s}\right)$ and $\hat{P}_{b}\left(\mathbf{v}_{s}\right)$.

\subsubsection{Asymptotic Analysis of Power Estimators}

The asymptotic analysis of power estimators $\hat{P}_{a}$ and $\hat{P}_{b}$ is performed in the limit when both the number of snapshots $n$ and the number of sensors $m$ grow large at the same rate so that $m / n \rightarrow c, c \neq 0$. The assumption that $m$ and $n$ grow at the same rate captures the fact that we often do not have enough snapshots to estimate accurately the input correlation. On the other hand, the assumption that $m, n \rightarrow \infty$ does not have practical justification, but is simply a mathematical necessity which enables analytical derivations.

The following lemma characterizes the asymptotic behavior of power estimators $\hat{P}_{a}$ and $\hat{P}_{b}$.

Lemma 3.4. Under assumptions 2 and 4 , in the limit when $m, n \rightarrow \infty$ at the same rate, i.e., $\frac{m}{n} \rightarrow c \in(0, \infty)$,

$$
\hat{P}_{a}\left(\mathbf{v}_{s}\right) \rightarrow \frac{\bar{Q}_{1}-\delta \bar{Q}_{2}}{\bar{Q}_{1}^{2}} \quad \text { a.s. }
$$

and

$$
\hat{P}_{b}\left(\mathbf{v}_{s}\right) \rightarrow \frac{1}{\bar{Q}_{1}} \quad \text { a.s. }
$$

where $\bar{Q}_{1}$ and $\bar{Q}_{2}$ are the quantities that the quadratic forms $Q_{1}$ and $Q_{2}$ converge to in the limit. They are given by

$$
\bar{Q}_{1}=\mathbf{v}_{s}^{H} \sum_{k=1}^{m} \frac{\mathbf{q}_{k} \mathbf{q}_{k}^{H}}{\lambda_{k}\left(1-c+c \delta \bar{M}_{1}\right)+\delta} \mathbf{v}_{s}
$$

and

$$
\bar{Q}_{2}=\mathbf{v}_{s}^{H} \sum_{k=1}^{m} \mathbf{q}_{k} \mathbf{q}_{k}^{H} \frac{\lambda_{k} c\left(\bar{M}_{1}-\delta \bar{M}_{2}\right)+1}{\left[\lambda_{k}\left(1-c+c \delta \bar{M}_{1}\right)+\delta\right]^{2}} \mathbf{v}_{s},
$$

where $\bar{M}_{1}$ and $\bar{M}_{2}$ are the limiting moments corresponding to diagonally loaded SCM of the rectangulary windowed snapshots. 
Proof. The convergence results (3.43) and (3.44) directly follow from the convergence of quadratic forms $Q_{1}$ and $Q_{2}$, and (3.14) and (3.15). The convergence of $Q_{1}$ and $Q_{2}$ is proved using Theorem 2.2.

The empirical Eigenvector Stieltjes Transforms corresponding to SCM $\hat{\mathbf{R}}$ and diagonally loaded SCM $\hat{\mathbf{R}}_{\delta}$ are defined with respect to the signal replica vector $\mathbf{v}_{s}$ (i.e., $\left.\mathbf{s}_{1}=\mathbf{s}_{2}=\mathbf{v}_{s}\right)$,

$$
\begin{aligned}
F_{\hat{\mathbf{R}}}(z) & =\mathbf{v}_{s}^{H}(\hat{\mathbf{R}}-z \mathbf{I})^{-1} \mathbf{v}_{s} \\
F_{\hat{\mathbf{R}}_{\delta}}(z) & =\mathbf{v}_{s}^{H}\left(\hat{\mathbf{R}}_{\delta}-z \mathbf{I}\right)^{-1} \mathbf{v}_{s} .
\end{aligned}
$$

These transforms are using (3.6) and (2.11) related as

$$
F_{\hat{\mathbf{R}}_{\delta}}(z)=F_{\hat{\mathbf{R}}}(z-\delta) .
$$

The existence and characterization of the limiting Eigenvector Stieltjes Transform corresponding to a random matrix model (3.16), used to describe the SCM $\hat{\mathbf{R}}$, is stated in Theorem 2.2. With the assumptions that the spectral norm of the ensemble correlation matrix $\mathbf{R}$ and norm of the signal replica vector $\mathbf{v}_{s}$ are uniformly bounded (assumptions 2 and 4), the conditions of Theorem 2.2 are met. Therefore,

$$
F_{\hat{\mathbf{R}}}(z) \rightarrow \bar{F}_{\hat{\mathbf{R}}}(z) \quad \text { a.s. },
$$

where

$$
\bar{F}_{\hat{\mathbf{R}}}(z)=\sum_{i=1}^{m} \frac{\mathbf{v}_{s}^{H} \mathbf{q}_{i} \mathbf{q}_{i}^{H} \mathbf{v}_{s}}{\lambda_{i}\left(1-c-c z \bar{S}_{\hat{\mathbf{R}}}(z)\right)-z} .
$$

Recall that $\bar{S}_{\hat{\mathbf{R}}}(z)$ is the limiting Stieltjes transform corresponding to the SCM $\hat{\mathbf{R}}$.

The existence of the limiting eigenvector Stieltjes transform directly follows from (3.49) and (3.50). It is characterized using (3.49) by

$$
\bar{F}_{\hat{\mathbf{R}}_{\delta}}(z)=\bar{F}_{\hat{\mathbf{R}}}(z-\delta) .
$$


The quadratic form $Q_{1}$ is related to the empirical eigenvector Stieltjes transform corresponding to diagonally loaded SCM as

$$
Q_{1}=\lim _{z \rightarrow 0^{-}} F_{\hat{\mathbf{R}}_{\delta}}(z)
$$

Recall that $z \rightarrow 0^{-}$compactly represents $\Im\{z\}=0$ and $\Re\{z\} \rightarrow 0^{-}$. Note that this limit exists because either $\delta>0$ or $n>m$ with zero probability of receiving two snapshots that are identical up to a scaling.

Hence, we conclude from (3.50) and (3.53) that the quadratic form $Q_{1}$ almost surely converges to non-random $\bar{Q}_{1}$, given by

$$
\bar{Q}_{1}=\lim _{z \rightarrow 0^{-}} \bar{F}_{\hat{\mathbf{R}}}(z-\delta)
$$

Finally, taking the above limit and recalling that the limiting moment

$$
\bar{M}_{1}=\lim _{z \rightarrow 0^{-}} \bar{S}_{\hat{\mathbf{R}}}(z-\delta)
$$

we obtain $(3.45)$.

The quadratic form $Q_{2}$ can be expressed in terms of the first derivative of the empirical Stieltjes transform corresponding to diagonally loaded SCM (provided it is analytic) as

$$
Q_{2}=\lim _{z \rightarrow 0^{-}} \frac{d}{d z} F_{\hat{\mathbf{R}}_{\delta}}(z)
$$

As already pointed pointed out, this limit exists because either $\delta>0$ or $n>m$ and the probability of receiving two snapshots identical up to a scaling is zero. Therefore, from (3.50) and (3.55), the quadratic form $Q_{2}$ almost surely converges to non-random $\bar{Q}_{2}$, given by

$$
\bar{Q}_{2}=\lim _{z \rightarrow 0^{-}} \frac{d}{d z} \bar{F}_{\hat{\mathbf{R}}}(z-\delta)
$$

Taking the above limit and recalling (3.54) and

$$
\bar{M}_{2}=\frac{1}{2} \lim _{z \rightarrow 0^{-}} \frac{\partial}{\partial z} \bar{S}_{\hat{\mathbf{R}}}(z-\delta)
$$


yields (3.46).

The limiting moments $\bar{M}_{1}$ and $\bar{M}_{2}$ in (3.45) and (3.46) are for diagonally loaded SCM evaluated from rectangularly windowed snapshots (observations) characterized in Section 2.5.2 and given as the solutions to the fixed point equations (2.51) and (2.52).

In comparison to the approach used here, note that [35] characterizes $\bar{Q}_{1}$ as a part of the study of the SINR at the MPDR output. The deterministic equivalent $\bar{Q}_{1}$ obtained therein is given in terms of the unique solution $B_{0}$ (in a certain set) to

$$
B_{0}=\frac{1}{m} \sum_{i=1}^{m} \frac{\lambda_{i}\left(1+c B_{0}\right)}{\lambda_{i}+\delta\left(1+c B_{0}\right)}
$$

Also, the study in [35] uses the true correlation $\mathbf{R}$ rather than $\hat{\mathbf{R}}$ in the definition of the power estimator (3.8). We believe that the estimator as defined in (3.8) is better suited for the spatial spectrum estimation.

\subsubsection{Asymptotically Unbiased Spatial Power Estimator}

The convergence result established in the previous part is used here to develop an asymptotically unbiased power estimator with respect to the alternatively defined true power (3.28).

First, assuming that the number of snapshots $n$ is greater than the number of sensors $m$, recall that the power estimators $\hat{P}_{a}$ and $\hat{P}_{b}$ are equal for diagonal loading $\delta=0$. Substituting $\delta=0$ in (3.44) yields that in the limit $m, n \rightarrow \infty$ such that $n>m$ and $\frac{m}{n} \rightarrow c \in(0,1)$

$$
\hat{P}\left(\mathbf{v}_{s}\right) \rightarrow \frac{1-c}{\mathbf{v}_{s}^{H} \mathbf{R}^{-1} \mathbf{v}_{s}} \quad \text { a.s. }
$$

Recalling that the estimated spatial power for known correlation matrix $\mathbf{R}$ is given by $1 / \mathbf{v}_{s}^{H} \mathbf{R}^{-1} \mathbf{v}_{s}$, we conclude that the unloaded power estimator is asymptotically biased with respect to the true power evaluated using (3.28). 
The asymptotically unbiased power estimator $\hat{P}^{\mathrm{ub}}\left(\mathbf{v}_{s}\right)$ is then obtained from (3.58) by dividing the unloaded estimator $\hat{P}_{b}\left(\mathbf{v}_{s}\right)$ with $1-c$ and is given by

$$
\hat{P}^{\mathrm{ub}}\left(\mathbf{v}_{s}\right)=\frac{1}{1-c} \frac{1}{\mathbf{v}_{s}^{H} \hat{\mathbf{R}} \mathbf{v}_{s}},
$$

where $m$ is the number of sensors, $n$ is the number of observations and $c=m / n$.

Note that while division by $(1-c)$ in $(3.59)$ produces an asymptotically unbiased estimator, the variance of the obtained estimator increases $1 /(1-c)^{2}$ times. This has detrimental effect on the quality of the estimation if the number of observations $n$ is only slightly higher than the number of sensors $m$. For example, if $c=0.9$, the variance increases 100 times!

\subsubsection{Approximate Expectation of Power Estimators}

As has been shown, both power estimators almost surely converge to corresponding deterministic quantities in the limit when $m, n \rightarrow \infty$ at the same rate such that $m / n \rightarrow c \in(0, \infty)$. According to the dominated convergence theorem [7], if power

estimators $\hat{P}_{a}$ and $\hat{P}_{b}$ are uniformly bounded as $m, n \rightarrow \infty$, their expectations converge to the same deterministic quantities. Indeed, under assumptions 2,3 and $4, \hat{P}_{a}$ is uniformly upper bounded for all $m$ and $n=n(m)$. Similarly, power estimator $\hat{P}_{b}$ is under same assumptions uniformly bounded for all $m$ and $n=n(m)$, provided that diagonal loading is finite. Therefore, the power estimators converge in expectation,

$$
\begin{aligned}
& \mathbf{E}\left[\hat{P}_{a}\left(\mathbf{v}_{s}\right)\right] \rightarrow \frac{\bar{Q}_{1}-\delta \bar{Q}_{2}}{\bar{Q}_{1}^{2}} \\
& \mathbf{E}\left[\hat{P}_{b}\left(\mathbf{v}_{s}\right)\right] \rightarrow \frac{1}{\bar{Q}_{1}}
\end{aligned}
$$

Although the established convergence results hold when $m, n \rightarrow \infty$ at the same rate such that $m / n \rightarrow c$, due to rapid convergence the asymptotic expressions accurately approximate the expectations of power estimators for relatively small $n$ and 
$m$. Therefore, for finite $n$ and $m$,

$$
\begin{aligned}
& \mathbf{E}\left[\hat{P}_{a}\left(\mathbf{v}_{s}\right)\right] \approx \frac{\bar{Q}_{1}-\delta \bar{Q}_{2}}{\bar{Q}_{1}^{2}} \\
& \mathbf{E}\left[\hat{P}_{b}\left(\mathbf{v}_{s}\right)\right] \approx \frac{1}{\bar{Q}_{1}},
\end{aligned}
$$

where $\bar{Q}_{1}$ and $\bar{Q}_{2}$ are evaluated using (3.45) and (3.46) with given $m, n$ and $c=m / n$.

These approximations are validated via simulations in the following part.

\subsubsection{Numerical Validation of Derived Expressions}

Approximations (3.62) and (3.63) are validated using Monte-Carlo simulations. We consider a standard, vertical, linear array with half-wavelength separation between elements. The MPDR algorithm is used to estimate directions of arrival and the corresponding powers. Spatially uncorrelated, zero-mean noise with a variance of one corrupts the signal snapshots. We point out that the derived characterizations hold for more general arrival process, ambient noise and array shapes.

The simulations and analytical expressions are compared on the expected estimated power versus angle of arrival plots for fixed diagonal loading value, as well as on the expected estimated power versus diagonal loading plots for fixed steering angle. Two different scenarios are considered.

\section{Scenario 1}

In the first scenario, 2 signals are arriving at elevation angles of $90^{\circ}$ and $92^{\circ}$ and each has power 10. The array has 30 sensors and 50 snapshots are used to estimate the sample correlation matrix. Note that the corresponding $c=0.6$ and the normalized trace of the ensemble correlation matrix is 21 .

The comparison between the mean of the estimate of the input power evaluated via simulations using (3.8) and the corresponding theoretical prediction (3.62) for the diagonal loading value of 0.3 is shown in the top part of Fig. 3-2. A similar agreement between the mean of power estimate (3.9) evaluated via simulations and theoretical 

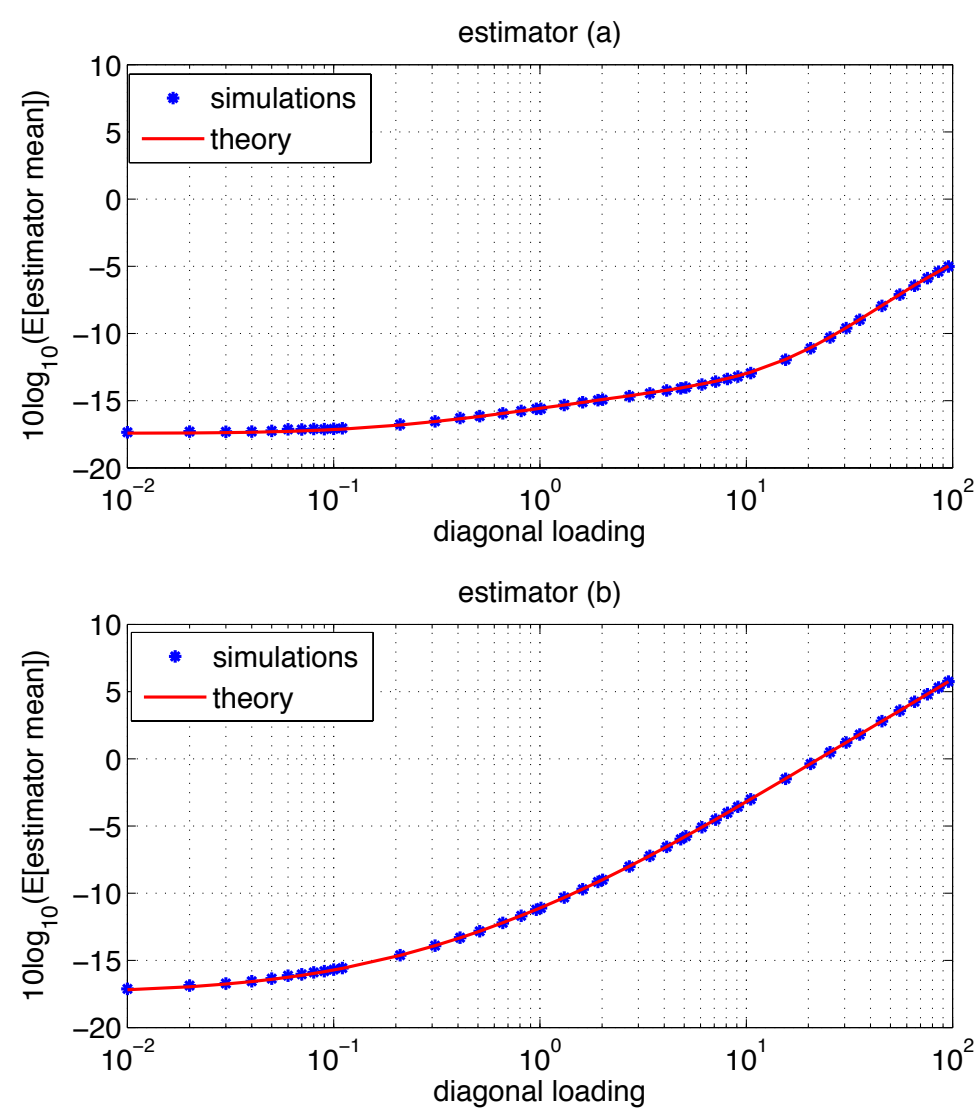

Figure 3-2: Scenario 1: Expected power versus diagonal loading for steering angle of $87^{\circ}$. The normalized trace of the corresponding ensemble correlation matrix is 21 .

prediction (3.63) for the same value of diagonal loading 0.3 is obtained in the bottom part of Fig. 3-2. Note that the diagonal loading of 0.3 is $1.43 \%$ of the normalized trace of the ensemble correlation matrix.

The comparisons between the simulated means of power estimators (3.8) and (3.9) and theoretical predictions (3.62) and (3.63) for a steering angle of $87^{\circ}$ are shown in Fig. 3-3. A good agreement between the plots validate the accuracy of the asymptotic results in predicting the expected values of the power estimators for finite values of $m$ and $n$. 

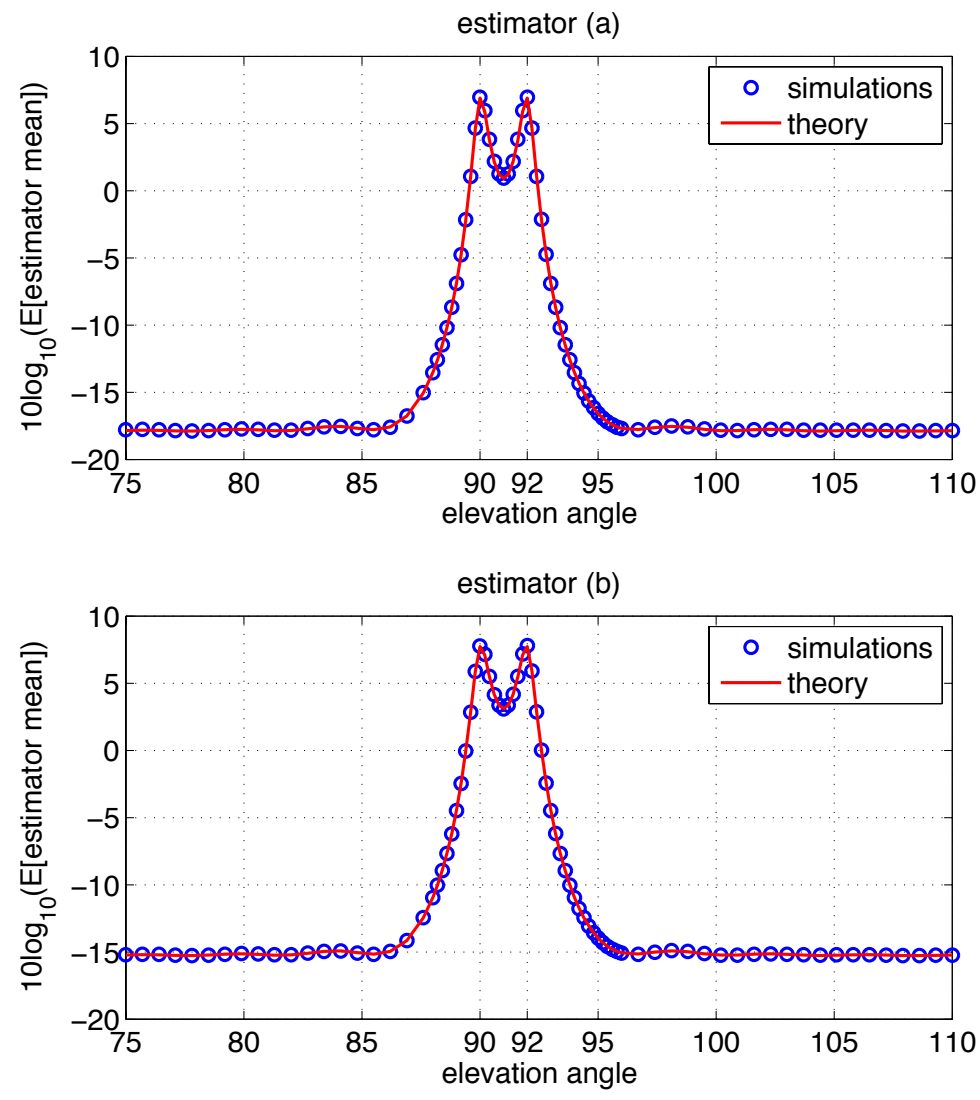

Figure 3-3: Scenario 1: Expected power versus steering angle for diagonal loading of 0.3 . This value of diagonal loading is $1.43 \%$ of the normalized trace of the ensemble correlation matrix. 


\section{Scenario 2}

In the second scenario, 2 signals are arriving at elevation angles of $90^{\circ}$ and $94^{\circ}$ and their SNR's are $1 \mathrm{~dB}$ and $5 \mathrm{~dB}$, respectively. The array has 40 sensors and 25 snapshots are used to estimate the sample correlation matrix. Note that the number of snapshots per sensor in this scenario is smaller than 1, i.e., $c=1.6$ and the normalized trace of the ensemble correlation matrix is 5.42 .

The comparisons between the expected values of $\hat{P}_{a}$ and $\hat{P}_{b}$, obtained from MonteCarlo simulations, and the corresponding theoretical predictions (3.62) and (3.63) for diagonal loading of 20 and varying steering direction and shown in Fig. 3-5. These comparisons are for steering direction of $87^{\circ}$ and varying diagonal loading shown in Fig. 3-4. The presented plots validate the accuracy of the theoretical predictions. Note that the normalized trace of the corresponding ensemble correlation matrix is

\subsection{Mean Square Error of Power Estimators}

As discussed in the Section 3.5.2, the asymptotically unbiased diagonally unloaded estimator might have large variance when the number of snapshots $n$ is only slightly greater than the number of sensors $m$. This motivates the study of variance and estimation mean square error. The variance and estimation MSE corresponding to power estimator $\hat{P}_{a}$ are evaluated in this section.

The received snapshots are assumed to be Gaussian distributed throughout this section. Using the Gaussian tools from Section 2.6, we evaluate the mean of the power

estimator $\hat{P}_{a}$ in the large $m, n$ limit. Although the asymptotic analysis of the means of power estimators has been conduced in the previous section, by restricting the process to be Gaussian distributed, we are able to characterize the order of convergence of the expectations of power estimators to their limiting values.

Furthermore, by imposing the Gaussian assumption on the received snapshots, we prove that the deviation of the power estimator $\hat{P}_{a}$ from its mean converges in distribution, when $m$ and $n$ grow large at the same rate, to the Gaussian random variable. The variance of the Gaussian distribution is evaluated and it relatively 

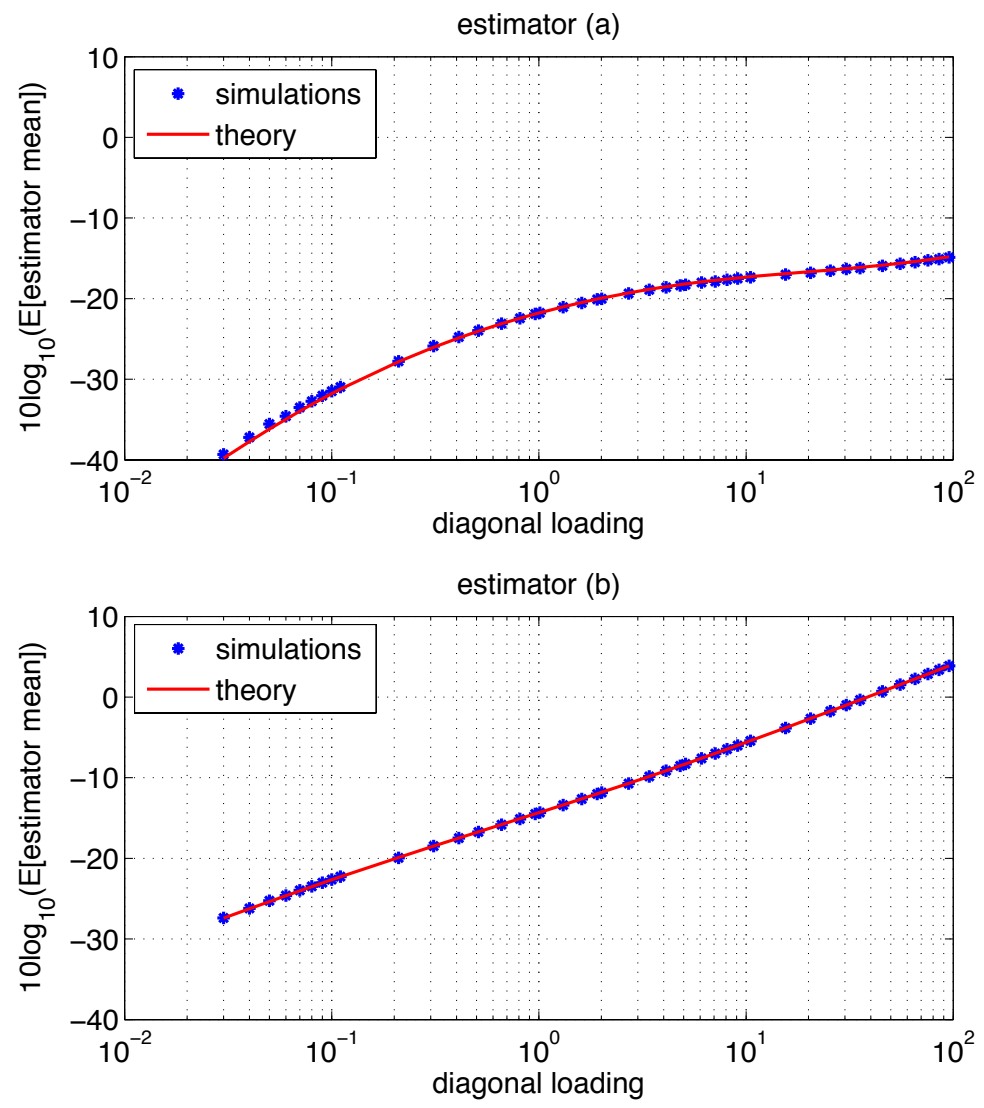

Figure 3-4: Scenario 2: Expected power versus diagonal loading for steering angle of $87^{\circ}$. The normalized trace of the ensemble correlation matrix is 5.42 . 

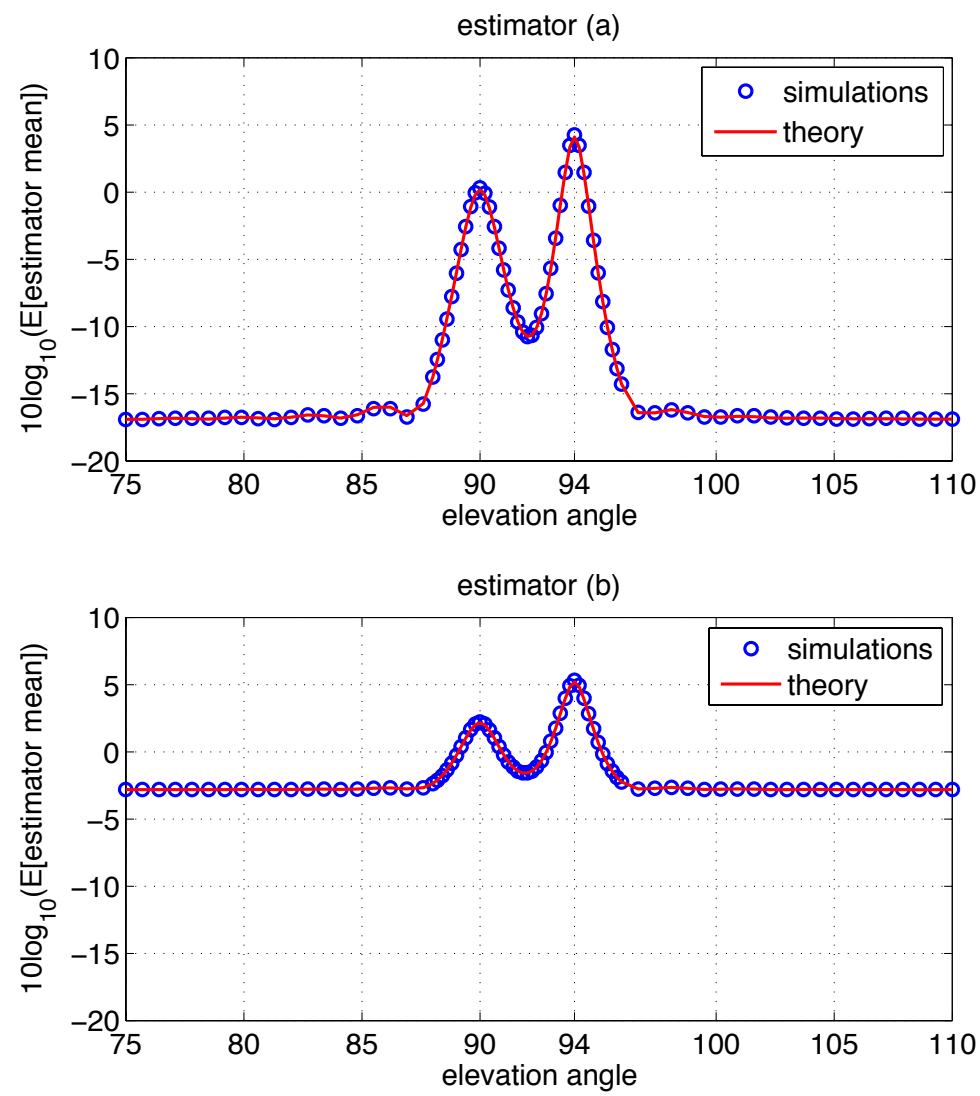

Figure 3-5: Scenario 2: Expected power versus steering angle for diagonal loading of 20. The normalized trace of the ensemble correlation matrix is 5.42 . 
accurately approximates the variance of the power estimator $\hat{P}_{a}$ for finite $m$ and $n$.

The method presented in this section closely follows the Gaussian Method introduced in [25], wherein the limiting behavior of the mutual information of MIMO channels is studied. This section outlines the steps used in characterizing the MSE performance of power estimator $\hat{P}_{a}$. The derived characterization is validated via Monte-Carlo simulations and these results are presented in the second part.

\subsubsection{Major Steps in the Derivation of Estimation MSE}

In short, the following analysis studies how the estimator $\hat{P}_{a}$ behaves in the limit when $m, n \rightarrow \infty$ such that $m / n \rightarrow c \in(0, \infty)$. In particular,

- We first evaluate the mean values of the quadratic forms $Q_{1}$ and $Q_{2}$, denoted respectively by $\mu_{1}$ and $\mu_{2}$. To keep the notation simple, the explicit dependence of the quadratic forms on diagonal loading $\delta$ is suppressed.

- Then, it is shown that the deviation of the vector $\mathbf{g}=\left[\begin{array}{ll}Q_{1} & Q_{2}\end{array}\right]^{T}$ from its mean $\mathbf{g}=\left[\begin{array}{ll}\mu_{1} & \mu_{2}\end{array}\right]^{T}$ converges in distribution, in large $m, n$ limit, to the Gaussian random variable. The covariance matrix $\boldsymbol{\Sigma}$ of the limiting distribution is computed.

- Finally, it is obtained that the estimator $\hat{P}_{a}$ is approximately Gaussian distributed. The mean value and variance of the approximating distribution are evaluated.

\section{Evaluation of $\mu_{1}$ : Step 1}

This and the subsequent part analyze behavior of the first moment of the quadratic form $Q_{1}$. Their purpose is to show how the Gaussian tools work in practice and to give a glimpse of how other quantities important for characterizing $\hat{P}_{a}$ are evaluated.

As a starting point, we introduce a resolvent identity, directly obtained from the definition of $\mathbf{H}$ in $(3.22)$

$$
\mathbf{H}=\mathbf{I}-\frac{t}{n} \mathbf{H Y} \mathbf{Y}^{H}
$$


Since $\mathbf{s}=\mathbf{Q}^{H} \mathbf{v}_{s}$ is deterministic,

$$
\mathbf{E}\left[t \mathbf{s}^{H} \mathbf{H s}\right]=t \mathbf{s}^{H} \mathbf{E}[\mathbf{H}] \mathbf{s}
$$

It follows from (3.64) that

$$
\mathbf{E}\left[H_{i j}\right]=\delta_{D}(i-j)-\frac{t}{n} \mathbf{E}\left[\left(H Y Y^{H}\right)_{i j}\right]
$$

The second term in (3.66) is given by

$$
\mathbf{E}\left[\left(H Y Y^{H}\right)_{i j}\right]=\sum_{k=1}^{m} \sum_{l=1}^{n} \mathbf{E}\left[H_{i k} Y_{k l} Y_{j l}^{*}\right]
$$

Using the integration by parts formula (2.66) with $f(\mathbf{Y})=H_{i k} Y_{j l}^{*}$, the summand in (3.67) is expressed as

$$
\mathbf{E}\left[H_{i k} Y_{k l} Y_{j l}^{*}\right]=\lambda_{k} \mathbf{E}\left[\frac{\partial\left(H_{i k} Y_{j l}^{*}\right)}{\partial Y_{k l}^{*}}\right]
$$

The derivative of the resolvent $\mathbf{H}$ is for (any) indices $i, j, k$ and $l$ given by [25]

$$
\frac{\partial H_{i j}}{\partial Y_{k l}^{*}}=-\frac{t}{n}(H Y)_{i l} H_{k j}
$$

Substituting (3.69) into (3.68) yields

$$
\mathbf{E}\left[H_{i k} Y_{k l} Y_{j l}^{*}\right]=\lambda_{k} \mathbf{E}\left[H_{i j} \delta_{D}(j-k)-\frac{t}{n}(H Y)_{i l} H_{k k} Y_{j l}^{*}\right]
$$

Now, summing (3.70) over $k$ yields

$$
\mathbf{E}\left[(H Y)_{i l} Y_{j l}^{*}\right]=\mathbf{E}\left[\lambda_{j} H_{i j}-\frac{t}{n} \operatorname{tr}\{\mathbf{D H}\}(H Y)_{i l} Y_{j l}^{*}\right] .
$$

Introducing $\beta=\frac{1}{n} \operatorname{tr}\{\mathbf{D H}\}$, expressing it as $\beta=\alpha+\hat{\beta}$ where $\alpha=\mathbf{E}[\beta]$ and $\mathbf{E}[\hat{\beta}]=0$, 
and substituting into (3.71) yields

$$
(1+t \alpha) \mathbf{E}\left[(H Y)_{i l} Y_{j l}^{*}\right]=\mathbf{E}\left[\lambda_{j} H_{i j}-t \hat{\beta}(H Y)_{i l} Y_{j l}^{*}\right]
$$

Summing (3.72) over $l$ leads to

$$
(1+t \alpha) \mathbf{E}\left[\left(H Y Y^{H}\right)_{i j}\right]=n \lambda_{j} \mathbf{E}\left[H_{i j}\right]-t \mathbf{E}\left[\hat{\beta}\left(H Y Y^{H}\right)_{i j}\right]
$$

Dividing (3.73) with $1+t \alpha$, substituting the resulting expression into (3.66) and after simple algebraic manipulations we obtain

$$
\mathbf{E}\left[H_{i j}\right]=g_{j} \delta_{D}(i, j)+\frac{t^{2} g_{j}}{n(1+t \alpha)} \mathbf{E}\left[\hat{\beta}\left(H Y Y^{H}\right)_{i j}\right],
$$

where $g_{j}=\frac{1+t \alpha}{1+t\left(\alpha+\lambda_{j}\right)}$. Note that $g_{j}$ 's are uniformly bounded because the eigenvalues $\lambda_{j}$ 's are upper bounded (according to assumption 2). Finally, substituting (3.74) into (3.65) yields

$$
\mathbf{E}\left[Q_{1}\right]=t \mathbf{s}^{H} \mathbf{G s}+\frac{t^{3}}{n(1+t \alpha)} \mathbf{E}\left[\hat{\beta} \mathbf{s}^{H} \mathbf{H} \mathbf{Y} \mathbf{Y}^{H} \mathbf{G s}\right]
$$

where $\mathbf{G}=\operatorname{diag}\left\{g_{1}, g_{2}, \ldots, g_{m}\right\}$. Using a similar reasoning as in [25], it can be proven that

$$
\mathbf{s}^{H} \mathbf{G} \mathbf{s}=\mathbf{s}^{H} \mathbf{T s}+O\left(\frac{1}{n}\right)
$$

where $\mathbf{T}=(\mathbf{I}+t y \mathbf{D})^{-1}$ and $y$ is a unique and positive solution of the non-linear equation

$$
1+\frac{t}{n} \sum_{i=1}^{m} \frac{\lambda_{i}}{1+t \lambda_{i} y}=\frac{1}{y}
$$

To complete the evaluation of $\mu_{1}$, it remains to characterize the second term in (3.75). In fact, the following part shows that this term decays as $1 / n$. In doing so, the Poincare-Nesh inequality (2.67) is exploited. 


\section{Evaluation of $\mu_{1}$ : Step 2}

The second term in (3.75), denoted by $\tilde{Q}_{1}$, is after introducing

$$
\Phi=\frac{1}{n^{2}} \mathbf{s}^{H} \mathbf{H} \mathbf{Y} \mathbf{Y}^{H} \mathbf{G s}
$$

compactly written as

$$
\tilde{Q}_{1}=n K \mathbf{E}[\hat{\beta} \Phi]=n K \mathbf{E}[\hat{\beta} \hat{\Phi}],
$$

where $\hat{\Phi}=\Phi-\mathbf{E}[\Phi]$ and $K=\frac{t^{3}}{1+t \alpha}=O(1)$. From the Cauchy-Schwartz inequality,

$$
\tilde{Q}_{1} \leq n K \sqrt{\operatorname{var}(\beta)} \sqrt{\operatorname{var}(\Phi)}
$$

It has been shown in [25] that $\operatorname{var}(\beta)=O\left(n^{-2}\right)$. In the following, we prove that $\operatorname{var}(\Phi)=O\left(n^{-2}\right)$.

Applying the Poincare-Nesh inequality (2.67) on functional $\Phi$ yields

$$
\operatorname{var}(\Phi) \leq \sum_{i=1}^{m} \sum_{j=1}^{n} \lambda_{i} \mathbf{E}\left[\left|\frac{\partial \Phi}{\partial Y_{i j}}\right|^{2}+\left|\frac{\partial \Phi}{\partial Y_{i j}^{*}}\right|^{2}\right]
$$

Unwrapping $\Phi$, taking its derivatives with respect to $Y_{i j}$ and $Y_{i j}^{*}$ and summing back yields

$$
\frac{\partial \Phi}{\partial Y_{i j}}=\frac{1}{n^{2}}\left(\Phi_{1}+\Phi_{2}\right) \quad \text { and } \quad \frac{\partial \Phi}{\partial Y_{i j}^{*}}=\frac{1}{n^{2}}\left(\Phi_{3}+\Phi_{4}\right)
$$

where

$$
\begin{aligned}
& \Phi_{1}=\left(\mathbf{s}^{H} \mathbf{H}\right)_{i}\left(\mathbf{Y}^{H} \mathbf{G s}\right)_{j}, \Phi_{2}=-\frac{t}{n}\left(\mathbf{s}^{H} \mathbf{H}\right)_{i}\left(\mathbf{Y}^{H} \mathbf{H Y G s}\right)_{j} \\
& \Phi_{3}=(\mathbf{G s})_{i}\left(\mathbf{s}^{H} \mathbf{H Y}\right)_{j} \Phi_{4}=-\frac{t}{n}\left(\mathbf{s}^{H} \mathbf{H Y}\right)_{j}\left(\mathbf{H Y Y} \mathbf{Y}^{H} \mathbf{G s}\right)_{i} .
\end{aligned}
$$

Using the inequality $(a+b)^{2} \leq 2\left(a^{2}+b^{2}\right)$, and substituting (3.82) into (3.81) yields

$$
\operatorname{var}(\Phi) \leq \sum_{i=1}^{m} \sum_{j=1}^{n} \frac{2 \lambda_{i}}{n^{4}} \mathbf{E}\left[\left|\Phi_{1}\right|^{2}+\left|\Phi_{2}\right|^{2}+\left|\Phi_{3}\right|^{2}+\left|\Phi_{4}\right|^{2}\right]
$$


Now, we need to upper bound each expectation in (3.83). Here, we evaluate an upper bound of the term involving $\Phi_{2}$. Namely,

$$
\begin{aligned}
& \sum_{i=1}^{m} \sum_{j=1}^{n} \frac{2 \lambda_{i}}{n^{4}} \mathbf{E}\left[\left|\Phi_{2}\right|^{2}\right] \\
& =\frac{2 t^{2}}{n^{6}} \mathbf{E}\left[\mathbf{s}^{H} \mathbf{G} \mathbf{Y} \mathbf{Y}^{H} \mathbf{H D H Y} \mathbf{Y}^{H} \mathbf{G} \mathbf{s s}^{H} \mathbf{H} \mathbf{Y} \mathbf{Y}^{\mathbf{H}} \mathbf{H} \mathbf{s}\right] \\
& \stackrel{(a)}{\leq} \frac{2 t^{2}}{n^{6}} \mathbf{E}\left[\|\mathbf{s}\|^{4}\|\mathbf{G}\|^{2}\|\mathbf{H}\|^{4}\|\mathbf{D}\|\left\|\left(\mathbf{Y} \mathbf{Y}^{H}\right)^{2}\right\|\left\|\mathbf{Y} \mathbf{Y}^{H}\right\|\right] \\
& \stackrel{(b)}{\leq} \frac{2 t^{2}}{n^{6}} K \mathbf{E}\left[\sqrt{\operatorname{tr}\left(\left(\mathbf{Y} \mathbf{Y}^{H}\right)^{4}\right)} \sqrt{\operatorname{tr}\left(\left(\mathbf{Y} \mathbf{Y}^{H}\right)^{2}\right)}\right] \\
& \left.\left.\stackrel{(c)}{\leq} \frac{K}{n^{2}} \sqrt{\mathbf{E}\left[\frac { 1 } { n } \operatorname { t r } \left(\left(\frac{\mathbf{Y} \mathbf{Y}^{H}}{n}\right)\right.\right.}\right)\right] \sqrt{\mathbf{E}\left[\frac{1}{n} \operatorname{tr}\left(\left(\frac{\mathbf{Y} \mathbf{Y}^{H}}{n}\right)^{2}\right)\right]} \\
& \stackrel{(d)}{=} O\left(\frac{1}{n^{2}}\right),
\end{aligned}
$$

where $\stackrel{(a)}{\leq}$ and $\stackrel{(c)}{\leq}$ follow from the Cauchy-Schwartz inequality. Inequality $\stackrel{(b)}{\leq}$ follows from the definition of the matrix norm and facts that each of $\mathbf{s}, \mathbf{D}, \mathbf{H}$ and $\mathbf{G}$ are uniformly bounded in norm because respectively, $\mathbf{s}$ is a unitary rotation of the array manifold vector, the largest eigenvalue of $\mathbf{R}$ is uniformly bounded by assumption 2 ., and by definition, $\|\mathbf{H}\| \leq 1$ and $\|\mathbf{G}\| \leq G_{\max }<\infty$. Finally, $\stackrel{(d)}{=}$ follows from the fact that $\mathbf{E}\left[\frac{1}{n} \operatorname{tr}\left(\left(\frac{\mathbf{Y Y}^{H}}{n}\right)^{k}\right)\right]=O(1)$ for any integer $k[25]$. The similar reasoning is applied for other terms in (3.82).

Finally from (3.80) and (3.84), we conclude that $\tilde{Q}_{1}=O\left(n^{-1}\right)$. Substituting this result and (3.76) into (3.75) yields

$$
\mathbf{E}\left[Q_{1}\right]=t \mathbf{s}^{H} \mathbf{T s}+O\left(n^{-1}\right) .
$$

The method presented in this and the previous part is general in the sense that it can be used to characterize first moments of different functionals of the resolvent matrix $\mathbf{H}$. 


\section{Statistics of the Vector $\mathrm{g}$}

This part outlines a method used to prove that the deviation of $\mathbf{g}$ from its mean $\overline{\mathbf{g}}$ is Gaussian distributed in the limit when $m, n \rightarrow \infty$ such that $m / n \rightarrow c \in(0, \infty)$. The covariance $\boldsymbol{\Sigma}$ of the limiting distribution is given by

$$
\boldsymbol{\Sigma}=\left[\begin{array}{ll}
\sigma_{1}^{2} & \sigma_{12} \\
\sigma_{12} & \sigma_{2}^{2}
\end{array}\right]
$$

where $\sigma_{1}^{2}, \sigma_{2}^{2}$ and $\sigma_{12}$ are respectively the variances and covariance of/between $Q_{1}$ and $Q_{2}$.

We introduce a new random variable $Q$ as a linear combination of the centered $Q_{1}$ and $Q_{2}$, namely

$$
Q=A\left(Q_{1}-\mu_{1}\right)+B\left(Q_{2}-\mu_{2}\right),
$$

where $\mu_{1}$ and $\mu_{2}$ are the expectations of the limiting distributions of $Q_{1}$ and $Q_{2}$. Note that $\mu_{1}$ has been evaluated in the previous part. The variance of $Q$ is given by

$$
\sigma_{Q}^{2}=A^{2} \sigma_{1}^{2}+B^{2} \sigma_{2}^{2}+2 A B \sigma_{12} .
$$

Note that the variances and covariance of/between $Q_{1}$ and $Q_{2}$ can be identified from $\sigma_{Q}^{2}$ by inspection.

Further, we denote a characteristic function of $Q$ by $\Psi$ and resort to the fact that if in the limit

$$
\Psi=\mathbf{E}\left[e^{j \omega Q}\right] \rightarrow e^{-\frac{\omega^{2} \sigma_{Q}^{2}}{2}},
$$

then, in distribution,

$$
\sigma_{Q}^{-1}(Q-\bar{Q}) \rightarrow \mathcal{N}(0,1) .
$$

In other words, if the characteristic function of $Q$ converges to that of a Gaussian random variable, then $Q$ itself is Gaussian distributed in the limit. The conver- 
gence (3.89) is established by showing that (details in [25])

$$
\frac{\partial \Psi}{\partial \omega}=-\omega \sigma_{Q}^{2} \Psi+\epsilon
$$

where $\epsilon$ converges to zero in the limit. In addition, $\sigma_{Q}^{2}$ is recovered from (3.91).

To establish (3.91), we start by taking the first derivative of $\Psi$

$$
\frac{\partial \Psi}{\partial \omega}=j A \mathbf{E}\left[Q_{1} e^{j \omega Q}\right]+j B \mathbf{E}\left[Q_{2} e^{j \omega Q}\right]-j \Psi\left(A \mu_{1}+B \mu_{2}\right) .
$$

In the further development, we evaluate $\mathbf{E}\left[Q_{k} e^{j \omega Q}\right]$ for $k=1$ and $k=2$. This is essentially done by using the integration by parts formula (2.66) and PoincareNash inequality (2.67). After a long interplay between these two Gaussian tools, Cauchy-Schwartz inequality and messy algebra, we obtain (3.91) and extract $\sigma_{Q}^{2}$. The components of $\sigma_{Q}^{2}$ are evaluated using (3.88) as

$$
\begin{aligned}
\sigma_{1}^{2}= & \frac{t^{4}}{n^{2}} \mathbf{E}\left[\mathbf{s}^{H} \mathbf{H D H} \mathbf{s}\right] \mathbf{E}\left[\mathbf{s}^{H} \mathbf{H} \mathbf{Y} \mathbf{Y}^{H} \mathbf{G s}\right] \\
\sigma_{2}^{2}= & \frac{t^{6}}{n^{2}}\left\{\mathbf { E } \left[\mathbf{s}^{H} \mathbf{H D H} \mathbf{H}^{2} \mathbf{\mathbf { s }}\left[\mathbf{s}^{H} \mathbf{H Y Y} \mathbf{Y}^{H} \mathbf{G}(\mathbf{I}+\mathbf{H}) \mathbf{s}\right]\right.\right. \\
& \left.+\mathbf{E}\left[\mathbf{s}^{H} \mathbf{H D H} \mathbf{\mathbf { s }}\right] \mathbf{E}\left[\mathbf{s}^{H} \mathbf{H Y Y} \mathbf{Y}^{H} \mathbf{G}(\mathbf{I}+\mathbf{H}) \mathbf{s}\right]\right\} \\
\sigma_{12}= & \frac{t^{5}}{2 n^{2}}\left\{\mathbf{E}\left[\mathbf{s}^{H} \mathbf{H D H} \mathbf{H}^{2} \mathbf{s}\right] \mathbf{E}\left[\mathbf{s}^{H} \mathbf{H Y Y} \mathbf{Y}^{H} \mathbf{G s}\right]\right. \\
& +\mathbf{E}\left[\mathbf{s}^{H} \mathbf{H D H s}\right] \mathbf{E}\left[\mathbf{s}^{H} \mathbf{H}^{2} \mathbf{Y} \mathbf{Y}^{H} \mathbf{G s}\right] \\
& \left.+\mathbf{E}\left[\mathbf{s}^{H} \mathbf{H D H s}\right] \mathbf{E}\left[\mathbf{s}^{H} \mathbf{H} \mathbf{Y} \mathbf{Y}^{H} \mathbf{G}(\mathbf{I}+\mathbf{H}) \mathbf{s}\right]\right\}
\end{aligned}
$$

The expectations in the above expressions are evaluated using the Gaussian tools. The resulting expressions admit closed forms in terms of $y$ and traces and quadratic forms involving diagonal matrices $\mathbf{D}$ and $\mathbf{T}$. The only difficulty is to solve a non-linear equation (3.77) for $y$, while the other quantities are easily calculated. We omit the presentation of the final expressions.

Having established (3.91), we conclude that when $m, n \rightarrow \infty$ such that $m / n \rightarrow$ $c \in(0, \infty), Q$ has Gaussian distribution with mean $\bar{Q}=A \mu_{1}+B \mu_{2}$ and variance $\sigma_{Q}^{2}$. 
Similarly, the vector $\mathbf{Q}$ is also Gaussian distributed in the limit, i.e.,

$$
\Sigma^{-1}(\mathbf{g}-\overline{\mathbf{g}}) \rightarrow \mathcal{N}(\mathbf{0}, \mathbf{I})
$$

where $\overline{\mathbf{g}}=\left[\begin{array}{ll}\mu_{1} & \mu_{2}\end{array}\right]^{T}$ is the expectation of the limiting Gaussian. The covariance matrix $\boldsymbol{\Sigma}$ of the limiting distribution is evaluated using (3.86).

The final major step in the statistical characterization of $\hat{P}_{a}$ is outlined in the following part.

\section{Statistical Characterization of $\hat{P}_{a}$}

Having established (3.93), we use the Delta method [25] to conclude that in the limit when $m, n \rightarrow \infty$ such that $m / n \rightarrow c \in(0, \infty)$,

$$
\sigma_{\hat{P}_{a}}^{-1}\left(\hat{P}_{a}-\mu_{\hat{P}_{a}}\right) \rightarrow \mathcal{N}(0,1)
$$

in distribution, where $\mu_{\hat{P}_{a}}$ and $\sigma_{\hat{P}_{a}}^{2}$ are the mean value and variance of the limiting Gaussian distribution. The mean value is evaluated as

$$
\mu_{\hat{P}_{a}}=\frac{t \mu_{1}-\mu_{2}}{t \mu_{1}^{2}}
$$

On the other hand, the variance is evaluated using

$$
\sigma_{\hat{P}_{a}}^{2}=\nabla \hat{P}_{a}\left(\mu_{1}, \mu_{2}\right) \Sigma \nabla \hat{P}_{a}^{T}\left(\mu_{1}, \mu_{2}\right)
$$

where $\nabla \hat{P}_{a}\left(\mu_{1}, \mu_{2}\right)$ is a gradient of $\hat{P}$, evaluated at the point $\left(\mu_{1}, \mu_{2}\right)$, i.e.,

$$
\nabla \hat{P}_{a}\left(\mu_{1}, \mu_{2}\right)=\left[\begin{array}{ll}
\frac{\partial \hat{P}}{\partial Q_{1}}\left(\mu_{1}, \mu_{2}\right) & \frac{\partial \hat{P}}{\partial Q_{2}}\left(\mu_{1}, \mu_{2}\right)
\end{array}\right]
$$

Thus, by evaluating the derivatives of $\hat{P}_{a}$ with respect to $Q_{1}$ and $Q_{2}$ at $\left(\mu_{1}, \mu_{2}\right)$ and making the necessary substitutions in (3.95) and (3.96), the final expressions for $\mu_{\hat{P}_{a}}$ and $\sigma_{\hat{P}_{a}}^{2}$ are obtained. 
As a last step, we exploit the fact that (3.94) converges rapidly so that the expectation and variance of $\hat{P}_{a}$ are for finite $m$ and $n$ approximated as

$$
\mathbf{E}\left[\hat{P}_{a}\right] \approx \mu_{\hat{P}_{a}} \text { and } \operatorname{var}\left(\hat{P}_{a}\right) \approx \sigma_{\hat{P}_{a}}^{2}
$$

Finally, the MSE of $\hat{P}_{a}$ is evaluated by substituting (3.98) into (3.10).

\subsubsection{Numerical Validation of Derived Expressions}

The derived expression for the mean square error of the spatial power estimator $\hat{P}_{a}$ is validated using Monte-Carlo simulations. We consider a standard, vertical, linear array with $\lambda / 2$ separation of the elements. The received signal is composed of plane waves. The MPDR algorithm is used to estimate their directions of arrival and the corresponding powers. Spatially uncorrelated, zero-mean noise with a variance of one corrupts the signal snapshots. The true power is defined in a standard way using (3.27). Note that the derived characterization of estimation MSE holds for more general arrival process, ambient noise and array shapes.

\section{Scenario 1}

In this scenario, 2 signals are arriving at elevation angles of $90^{\circ}$ and $92^{\circ}$. Each signal has power 10. The array has 30 sensors and 50 snapshots are used to estimate the correlation matrix. Note that $c=0.6$ and the normalized trace of the ensemble correlation matrix is 21 .

The comparison between the dependences of simulated and theoretically predicted MSE's on diagonal loading for fixed steering direction is shown in Fig. 3-6. The plots in the top part correspond to steering away from the sources in the direction of $85^{\circ}$. The comparison in the bottom part corresponds to steering halfway between the sources in the direction of $91^{\circ}$. The true power is evaluated using the standard approach (3.27). The shown comparisons validate the accuracy of the derived theoretical prediction.

The simulation study has shown that the derived prediction of the MSE perfor- 

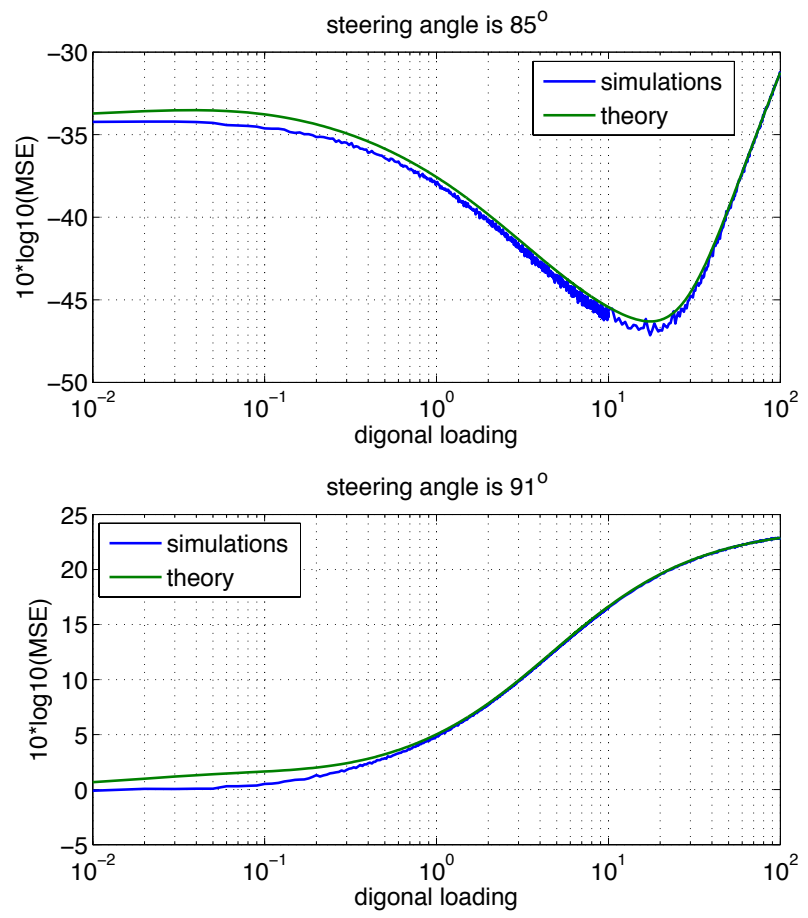

Figure 3-6: Scenario 1: MSE versus diagonal loading when steering angle is $85^{\circ}$ (top figure) and $91^{\circ}$ (bottom figure). True power uses the traditional definition as given in $(3.27)$.

mance is most sensitive around the values of optimal diagonal loading when steering close to sources. ${ }^{5}$ To further investigate this issue, the MSEs obtained via simulations and using the theoretical prediction are compared in Fig. 3-7. These MSE's in each steering direction are evaluated at the diagonal loading which minimizes the corresponding simulated MSE. As can be observed, the largest deviation happens when steering close to the source directions. As an aside note, it can be observed that the optimized MSE is relatively large when pointing at the source. Addressing this issue is a possible direction in future research.

\section{Scenario 2}

In this scenario, 2 signals are arriving at elevation angles of $90^{\circ}$ and $94^{\circ}$ and their SNR's are $1 \mathrm{~dB}$ and $5 \mathrm{~dB}$, respectively. The array has 40 sensors and 25 snapshots

\footnotetext{
${ }^{5}$ However, this is an important case especially when steering towards a weak source near a strong one.
} 


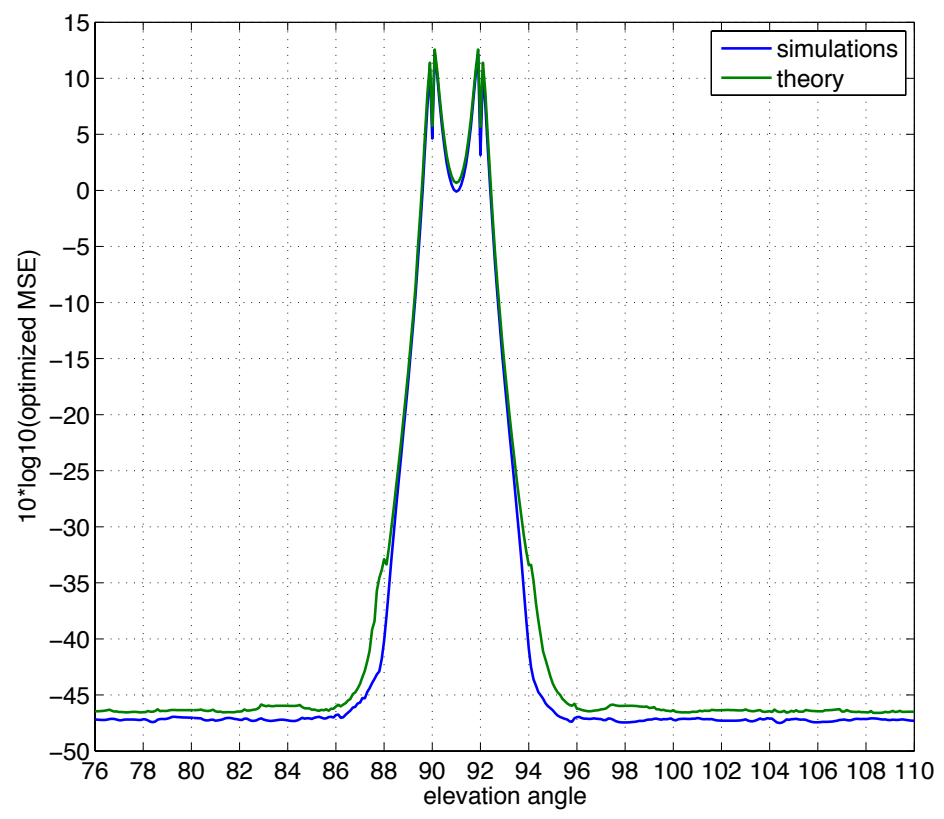

Figure 3-7: Scenario 1: Optimized MSE versus elevation angle. True power uses the traditional definition as given in (3.27).

are used to estimate the correlation matrix. Note that in this case the number of sensors is larger than the number of snapshots, i.e., $c=1.6$. Also, the normalized trace of the ensemble correlation matrix is 5.42 .

The comparisons between dependences of the simulated and theoretically predicted MSE's on diagonal loading for particular values of steering directions and true power defined in a standard way using (3.27) are shown in Fig. 3-8. The comparison in the top part corresponds to steering away from the sources in the direction of $87^{\circ}$, while that in the bottom part corresponds to steering halfway between the sources in the direction of $92^{\circ}$.

As in previous scenario, the theoretical prediction of the MSE performance is most sensitive when the steering close to sources and the diagonal loading is around the optimal diagonal loading. The comparison between the optimal MSE's evaluated via simulations and by using the theoretical prediction is shown in the top part of Fig. 3-9. The bottom part of Fig. 3-9 compares the dependences of the simulated and theoretically predicted MSE's on diagonal loading when steering very close to 

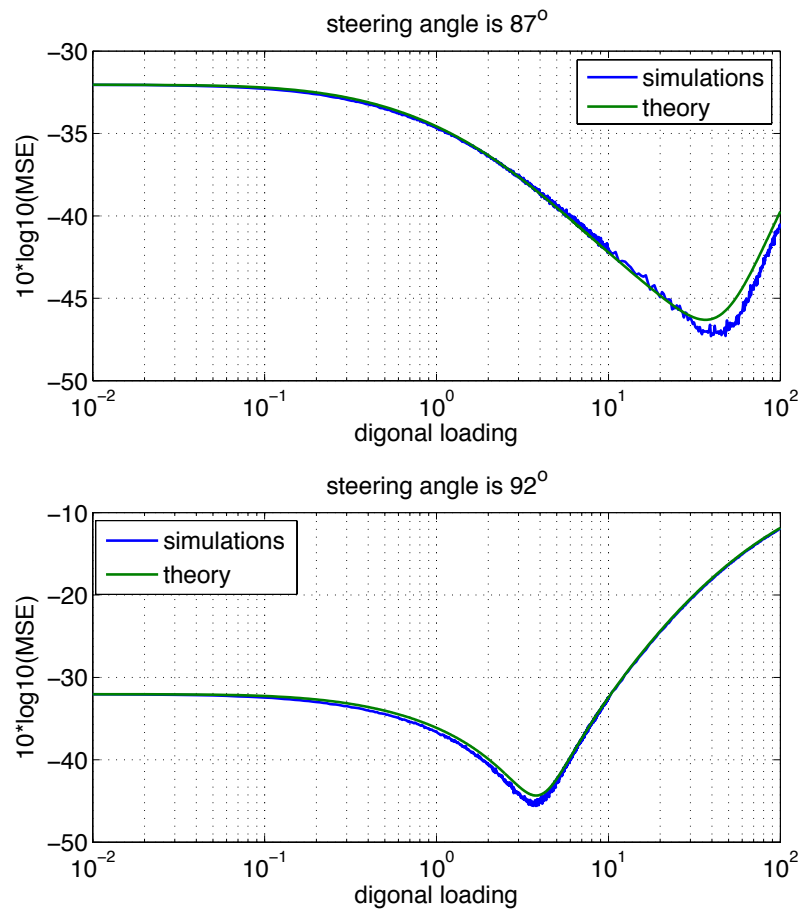

Figure 3-8: Scenario 2: MSE versus diagonal loading when steering angle is $87^{\circ}$ (top figure) and $92^{\circ}$ (bottom figure). True power uses the traditional definition as given in $(3.27)$.

the sources in direction of $89.5^{\circ}$. As can be observed from these comparisons, the theoretical prediction of the MSE is inaccurate when steering close to source directions and the diagonal loading is around the diagonal loading which optimizes the MSE performance. Finding the causes for this disagreement and improving the prediction is one possible future direction.

\subsection{Optimization of Mean Square Error}

Having established how the squared bias and variance depend on loading $\delta$, we turn our attention to studying how these quantities are interrelated. This section conjectures in the first part that the variance has insignificant impact on the diagonal loading which optimizes the estimation MSE performance. The numerical validation of this result is presented in the second part.

Throughout this section, we do not explicitly show the dependence of the power 

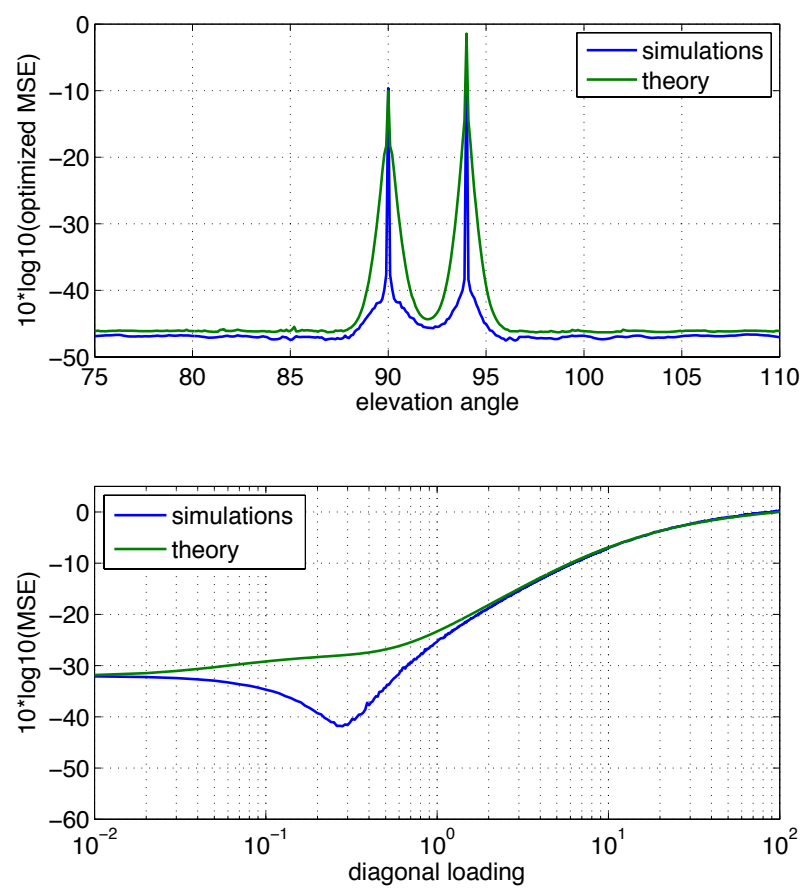

Figure 3-9: Scenario 2: Optimized MSE versus elevation angle (top figure). MSE versus diagonal loading for steering angle of $89.5^{\circ}$ (bottom figure). True power uses the traditional definition as given in (3.27). 
estimators on steering direction $\mathbf{v}_{s}$, i.e., with slight abuse of notation we write $\hat{P}(\delta)$.

\subsubsection{Estimation MSE versus Squared Bias Optimization}

In this part, we present arguments to support our conjecture that $\operatorname{var}(\hat{P}(\delta))$ has negligible impact on the value of optimal loading $\delta_{\text {opt }}$.

The following two lemmas establish bounds and orders of decay on first derivatives of power estimators and on variances and their first derivatives. The proofs are given in Appendices A and B.

Lemma 3.5. For a non-negative loading $\delta$ and under assumptions 2 and 3 , the following bounds hold almost surely

1. $\frac{\partial \hat{P}_{a}}{\partial \delta} \leq \frac{K(\delta)}{m}$

2. $\frac{C_{1}(\delta)}{m} \leq \frac{\partial \hat{P}_{b}}{\partial \delta} \leq \frac{C_{2}(\delta)}{m}$

where $K, C_{1}$ and $C_{2}$ are some positive constants.

Lemma 3.6. If the received snapshots $\mathbf{u}(i)$ are Gaussian distributed, then under assumptions 1-4, the following hold,

1. $\operatorname{var}\left(\hat{P}_{a}\right) \leq O\left(m^{-\frac{5}{2}}\right)$ and $\operatorname{var}\left(\hat{P}_{b}\right) \leq O\left(m^{-\frac{5}{2}}\right)$

2. $\operatorname{var}\left(\frac{\partial \hat{P}_{a}}{\partial \delta}\right) \leq O\left(m^{-\frac{5}{2}}\right)$ and $\operatorname{var}\left(\frac{\partial \hat{P}_{b}}{\partial \delta}\right) \leq O\left(m^{-\frac{5}{2}}\right)$.

Recall that the previous lemma holds if we replace $m$ with $n$ because $m$ and $n$ grow at the same rate according to assumption 1.

It can be concluded that the same bounds as established in Lemma 3.5 apply to the expectations of power estimators. In short, $\mathbf{E}\left[\frac{\partial \hat{P}(\delta)}{\partial \delta}\right]=O\left(m^{-1}\right)$. Note that the orders of decay established in Lemma 3.6 are not necessarily tight. Namely, Lemma 3.3 shows that if the input is Gaussian, $\delta \rightarrow \infty$ and $m$ and $n$ are of the same order, the variance of both estimators is $O\left(\mathrm{~m}^{-3}\right)$.

To conjecture that the variance has negligible impact on optimal loading, we first note that outside some region around a point at which the squared bias attains zero, 
the variance is significantly smaller than the squared bias. Namely, note that the expected values of both estimators are $O\left(m^{-1}\right)$ at $\delta=0$ and as $\delta \rightarrow \infty$, the estimator $\hat{P}_{a}$ is of $O\left(m^{-1}\right)$, while $\hat{P}_{b}$ is unbounded. On the other hand, the variances of both estimators are finite across whole $\delta$ region and of $O\left(m^{-\frac{5}{2}}\right)$.

Next we consider how the slopes of the variance and squared bias behave with respect to loading $\delta$. Starting from the former, the slope of the variance is expressed as the scaled correlation between the estimator and its slope and is upper bounded using the Cauchy-Schwartz inequality,

$$
\begin{aligned}
\frac{\partial}{\partial \delta} \operatorname{var}(\hat{P}) & =2 \operatorname{cov}\left(\hat{P}, \frac{\partial \hat{P}}{\partial \delta}\right) \\
& \leq 2 \sqrt{\operatorname{var}(\hat{P}) \operatorname{var}\left(\frac{\partial \hat{P}}{\partial \delta}\right)}
\end{aligned}
$$

The bounds on the variance of the estimator and its derivative are given in Lemma 3.6 and therefore the slope of the variance is upper bounded

$$
\frac{\partial}{\partial \delta} \operatorname{var}(\hat{P}) \leq O\left(m^{-\frac{5}{2}}\right)
$$

On the other hand, the slope of the squared bias is given by

$$
\frac{\partial}{\partial \delta} \operatorname{bias}^{2}(\delta)=2 \mathbf{E}[\hat{P}(\delta)-P] \mathbf{E}\left[\frac{\partial \hat{P}}{\partial \delta}\right]
$$

Therefore, the slope of the squared bias achieves zero at $\delta=\tilde{\delta}_{\text {opt }}$. Outside the region around a point at which it attains zero, the slope is of $O\left(\mathrm{~m}^{-1}\right)$. The slope of the squared bias corresponding to $\hat{P}_{a}$ is zero at $\delta=0$ and as $\delta \rightarrow \infty$. Overall, the slope of the squared bias varies more significantly than the slope of the variance.

To summarize,

1. The variance is significantly smaller than the squared bias outside some region around the point at which the squared bias equals zero.

2. The slope of the squared bias varies more significantly and is much larger than 
the slope of the variance.

The preceding two arguments imply that the variance has no significant impact on the value of optimal loading. In other words, the loss incurred by using $\tilde{\delta}_{\text {opt }}$ instead of $\delta_{\text {opt }}$ is negligible. This loss is quantified for spatial power estimator $\hat{P}_{b}$ in the following part.

\subsubsection{Estimation MSE Loss for Power Estimator $\hat{P}_{b}$}

Strictly speaking, $\tilde{\delta}_{\text {opt }}$ is not an optimal diagonal loading which minimizes the MSE. However, according to the arguments presented in the previous part, $\delta_{\text {opt }}$ and $\tilde{\delta}_{\text {opt }}$ are not far from each other. To make the argument more precise, we study how much the MSE is degraded by using $\tilde{\delta}_{\text {opt }}$ as an optimal loading. A MSE loss incurred by setting $\tilde{\delta}_{\text {opt }}$ instead of $\delta_{\text {opt }}$ is defined as

$$
L\left(\delta_{\mathrm{opt}}, \tilde{\delta}_{\mathrm{opt}}\right)=\operatorname{MSE}\left(\tilde{\delta}_{\mathrm{opt}}\right)-\operatorname{MSE}\left(\delta_{\mathrm{opt}}\right) .
$$

We show that in the large $m, n$ regime, the MSE loss $L$ becomes negligible compared to the optimal MSE. In doing so, we assume without loss of generality that the true power $P$ is such that the optimal loading $\delta_{\mathrm{opt}}$ is non-zero and finite.

The squared bias attains zero at $\tilde{\delta}_{\text {opt }}$. It is convex and approximately quadratic in a certain interval around $\tilde{\delta}_{\text {opt }}$. Therefore, the squared bias is within that interval approximated with a second order Taylor polynomial,

$$
\operatorname{bias}^{2}(\delta) \approx \frac{1}{2} \frac{\partial^{2} \operatorname{bias}^{2}\left(\tilde{\delta}_{\mathrm{opt}}\right)}{\partial \delta^{2}}\left(\delta-\tilde{\delta}_{\mathrm{opt}}\right)^{2}
$$

Invoke that $\operatorname{bias}^{2}\left(\tilde{\delta}_{\mathrm{opt}}\right)=0$ and $\frac{\partial}{\partial \delta} \operatorname{bias}^{2}\left(\tilde{\delta}_{\mathrm{opt}}\right)=0$. The second derivative of the squared bias at $\tilde{\delta}_{\text {opt }}$ is obtained by differentiating (3.101) and is given by

$$
\frac{\partial^{2} \operatorname{bias}^{2}\left(\tilde{\delta}_{\mathrm{opt}}\right)}{\partial \delta^{2}}=2 \mathbf{E}^{2}\left[\frac{\partial \hat{P}_{b}\left(\tilde{\delta}_{\mathrm{opt}}\right)}{\partial \delta}\right] .
$$


The squared bias is then approximated as

$$
\operatorname{bias}^{2}(\delta) \approx \mathbf{E}^{2}\left[\frac{\partial \hat{P}_{b}\left(\tilde{\delta}_{\mathrm{opt}}\right)}{\partial \delta}\right]\left(\delta-\tilde{\delta}_{\mathrm{opt}}\right)^{2}
$$

On the other hand, the variance is approximated with a first order Taylor polynomial around $\tilde{\delta}_{\text {opt }}$. This is because the variability in the slope of the variance is much smaller than that of the squared bias. Hence, using (3.99),

$$
\begin{aligned}
& \operatorname{var}\left(\hat{P}_{b}(\delta)\right) \approx \operatorname{var}\left(\hat{P}_{b}\left(\tilde{\delta}_{\mathrm{opt}}\right)\right)+\frac{\partial \operatorname{var}\left(\hat{P}_{b}\left(\tilde{\delta}_{\mathrm{opt}}\right)\right)}{\partial \delta}\left(\delta-\tilde{\delta}_{\mathrm{opt}}\right) \\
& =\operatorname{var}\left(\hat{P}_{b}\left(\tilde{\delta}_{\mathrm{opt}}\right)\right)+2 \operatorname{cov}\left(\hat{P}_{b}\left(\tilde{\delta}_{\mathrm{opt}}\right), \frac{\partial \hat{P}_{b}\left(\tilde{\delta}_{\mathrm{opt}}\right)}{\partial \delta}\right)\left(\delta-\tilde{\delta}_{\mathrm{opt}}\right) .
\end{aligned}
$$

Given that the squared bias and variance are approximated respectively with quadratic and linear functions, the problem of evaluating the MSE loss is boiled down to determining the loss incurred by declaring that the minimizer of the sum of these two functions is the argument which minimizes the quadratic function (Appendix C). Applying (C.3), this yields

$$
L\left(\delta_{\mathrm{opt}}, \tilde{\delta}_{\mathrm{opt}}\right) \approx \frac{\operatorname{cov}^{2}\left(\hat{P}_{b}\left(\tilde{\delta}_{\mathrm{opt}}\right), \frac{\partial \hat{P}_{b}\left(\tilde{\delta}_{\mathrm{opt}}\right)}{\partial \delta}\right)}{\mathbf{E}^{2}\left[\frac{\partial \hat{P}_{b}\left(\tilde{\delta}_{\mathrm{opt}}\right)}{\partial \delta}\right]} .
$$

The MSE loss (3.107) is upper bounded by invoking that $\frac{\partial \hat{P}_{\mathrm{b}}}{\partial \delta}>\frac{K}{m}$ from Lemma 3.5 and by utilizing (3.99) and (3.100). Therefore,

$$
L\left(\delta_{\mathrm{opt}}, \tilde{\delta}_{\mathrm{opt}}\right)=O\left(m^{-3}\right)
$$

Finally, in the limit when $m$ and $n$ grow large at the same rate,

$$
L\left(\delta_{\text {opt }}, \tilde{\delta}_{\text {opt }}\right) \ll \operatorname{MSE}\left(\delta_{\text {opt }}\right)
$$

i.e., the MSE loss is negligible compared to the optimum MSE in the large $(m, n)$ 
regime.

\subsubsection{Numerical Validation}

The presented results are validated via Monte-Carlo simulations. We consider different scenarios with respect to number of arrivals, their powers and directions of arrival.

A standard, vertical, linear array with a half-wavelength separation between the elements is considered in each scenario. In addition, a spatially uncorrelated, zeromean noise with a variance of one corrupts the signal snapshots.

\section{Scenario 1: Two Closely Spaced Arrivals with High SNR}

In this scenario, 2 signals are arriving at elevation angles of $90^{\circ}$ and $92^{\circ}$ and each has power 10 (i.e., the SNR is $10 \mathrm{~dB}$ ). The array contains 30 sensors and 50 snapshots are used to estimate the sample correlation matrix. Note that $c=0.6$ and the normalized trace of the ensemble correlation matrix is 21 .

The simulated dependence of the squared bias, variance and MSE on diagonal loading is shown in Fig. 3-10 for the true power defined in a standard way (3.27) and in Fig. 3-11 when the true power is defined using an alternative approach (3.28). A steering angle is $87^{\circ}$ and the plots show that the variance has almost no influence on a diagonal loading which minimizes the MSE.

The figures also show that the diagonal loading which optimizes the MSE corresponding to power estimator $\hat{P}_{b}$ is not greater than the loading which optimizes the MSE corresponding to $\hat{P}_{a}$. This is because both power estimators are monotonically non-decreasing functions of $\delta, \hat{P}_{b}(\delta) \geq \hat{P}_{a}(\delta)$ and the conjecture that the bias term controls the optimal diagonal loading.

Finally, note that the optimal loading minimizing either estimator when the true power is defined in a standard way is larger than the optimal loading when the power is defined in an alternative way. This is due to the combined effect of the facts that (1) both power estimators are non-decreasing functions of $\delta,(2)$ the true power evaluated 
with (3.28) is greater than the true power evaluated in a standard way using (3.27) and (3) our conjecture that bias controls the value of optimal diagonal loading.

A similar set of plots corresponding to steering angle of $89^{\circ}$ are given in Fig. $3-12$ and Fig. 3-13. As can be observed from Fig. 3-12, when the true power is defined in a standard way using (3.27), the optimal loading for both estimators is zero. This happens because the true power in such direction (and in general in directions sufficiently close to the directions of arrival) is below the expected smallest value of either power estimator, achieved for zero loading. Therefore, since the power estimators are non-decreasing functions of loading $\delta$, an unloaded power estimator minimizes the bias and hence, due to our conjecture, the estimation MSE. Intuitively, as the steering direction gets closer to but is not pointed exactly at the source, the optimal diagonal loading is reduced as the estimator needs to maintain more adaptability to null the source.

On the other hand, if the true power is evaluated using (3.28), the corresponding bias (and hence the estimation MSE) is minimized for non-zero loading because the expected smallest value of either power estimator is above the alternatively defined true power whenever $c<1$.

The optimal MSE and the MSE evaluated at a loading which minimizes the squared bias are compared in Fig. 3-14 for power estimator $\hat{P}_{a}$ and true power evaluated according to (3.27). The plots highlight two different ranges of steering angles. As shown, the performance loss is larger when steering away from the main beams, but remains within $1 \mathrm{~dB}$ of the optimal MSE. A smaller performance loss is observed in Fig. 3-15, which corresponds to the estimator $\hat{P}_{b}$.

The corresponding comparisons for power estimators $\hat{P}_{a}$ and $\hat{P}_{b}$ and true power evaluated using (3.28) are shown in Figures 3-16 and 3-17. Again, the largest performance loss is observed for power estimator $\hat{P}_{a}$ when steering away from the main beams and it remains within $1 \mathrm{~dB}$ of optimal MSE.

Finally, note that the optimized estimator $\hat{P}_{b}$ outperforms the optimized estimator $\hat{P}_{a}$. The comparison between the power estimators is given in Section 3.9. 

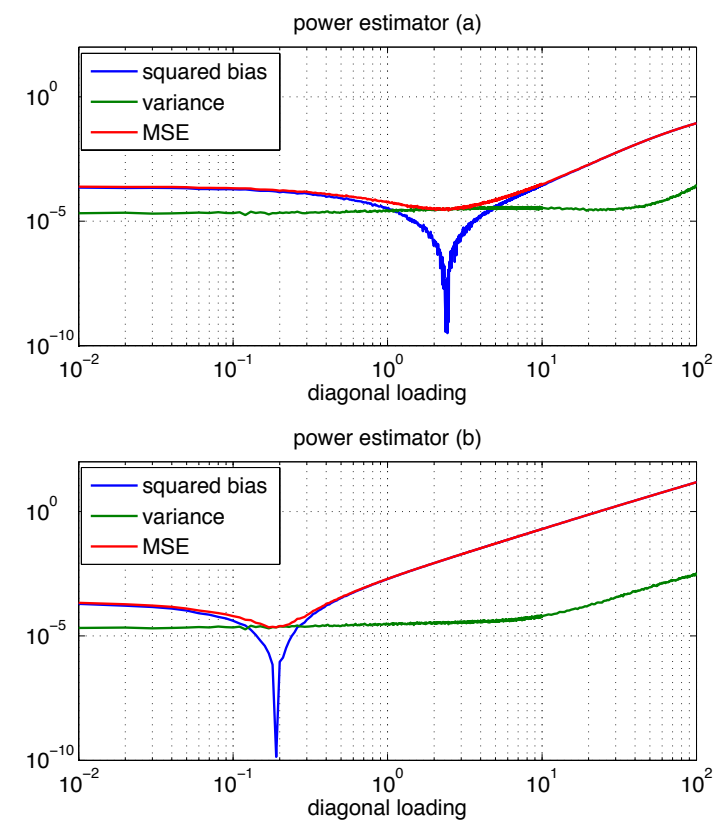

Figure 3-10: Scenario 1: Squared bias, variance and MSE corresponding to estimators $\hat{P}_{a}$ and $\hat{P}_{b}$ versus diagonal loading for steering angle of $87^{\circ}$. True power uses the traditional definition as given in (3.27).
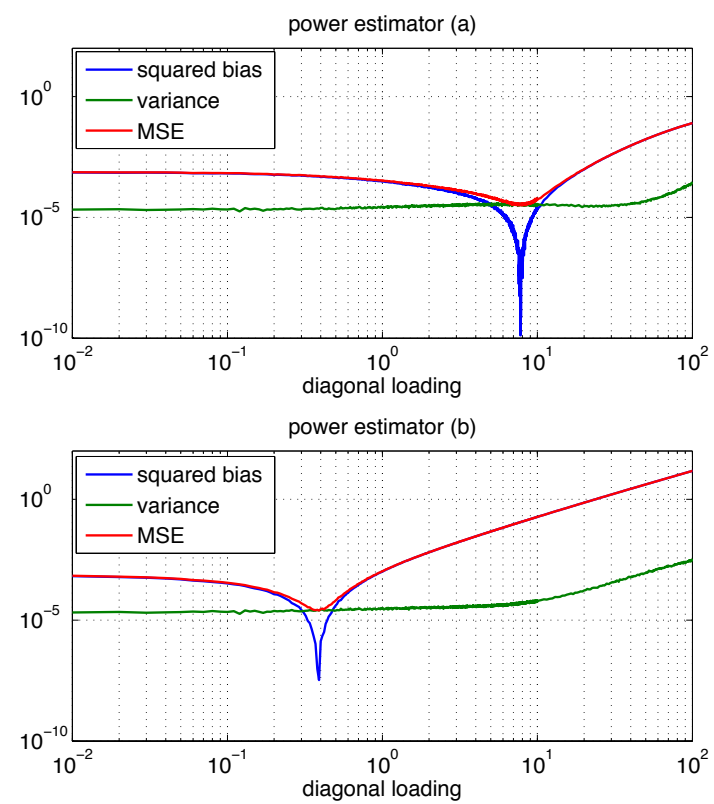

Figure 3-11: Scenario 1: Squared bias, variance and MSE corresponding to estimators $\hat{P}_{a}$ and $\hat{P}_{b}$ versus diagonal loading for steering angle of $87^{\circ}$. True power uses the alternative definition as given in (3.28). 

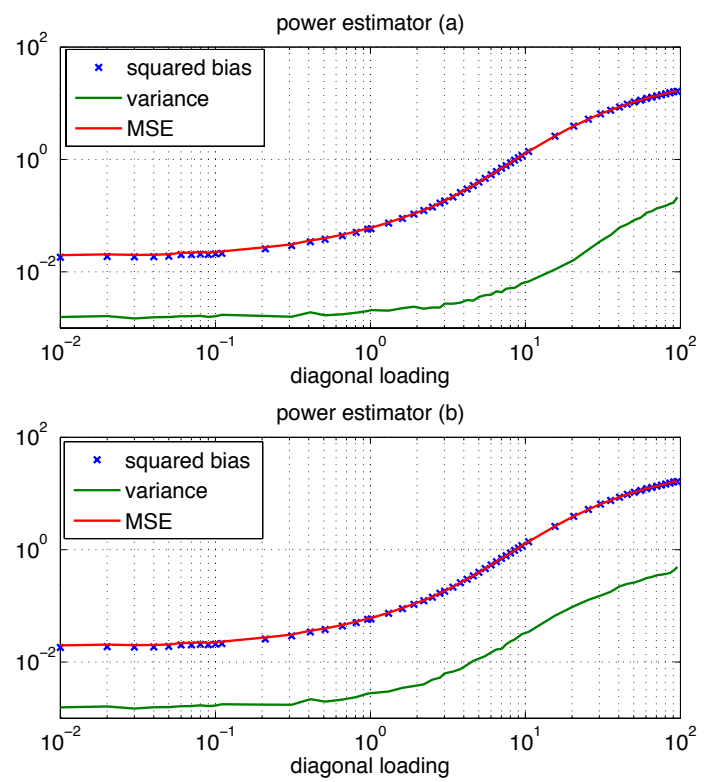

Figure 3-12: Scenario 1: Squared Bias, variance and MSE corresponding to estimators $\hat{P}_{a}$ and $\hat{P}_{b}$ versus diagonal loading for steering angle of $89^{\circ}$. True power uses the traditional definition as given in (3.27).
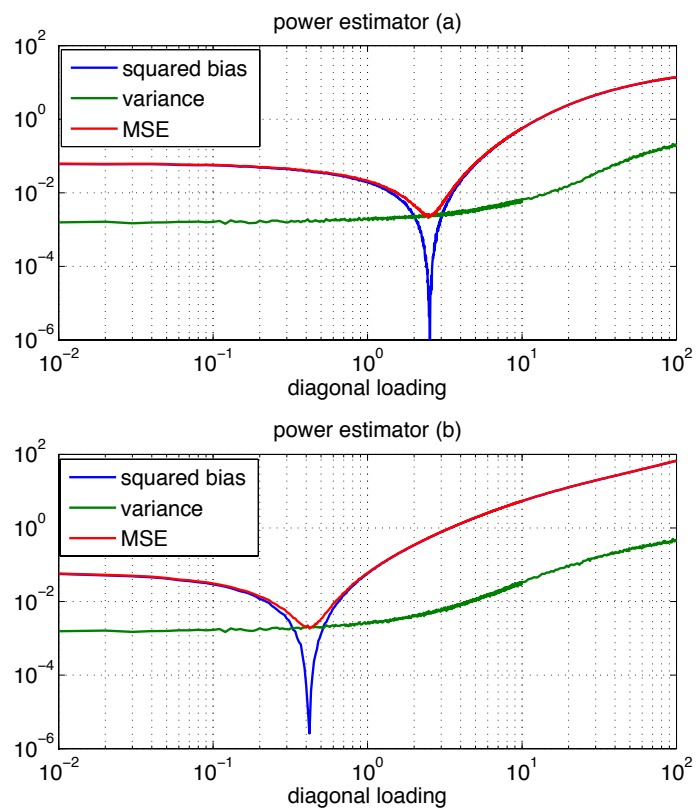

Figure 3-13: Scenario 1: Squared bias, variance and MSE corresponding to estimators $\hat{P}_{a}$ and $\hat{P}_{b}$ versus diagonal loading for steering angle of $89^{\circ}$. True power uses the alternative definition as given in (3.28). 

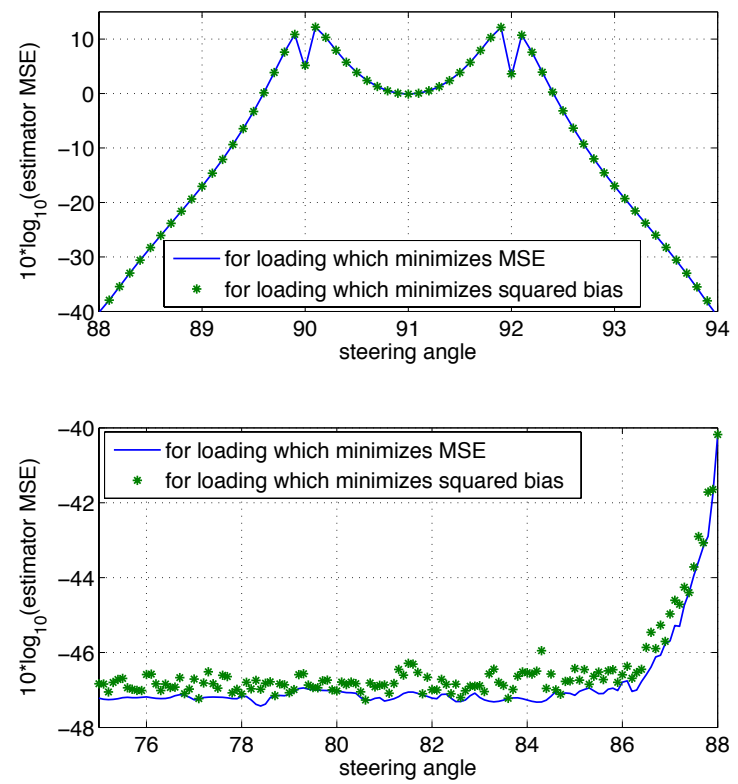

Figure 3-14: Scenario 1: $\operatorname{MSE}\left(\hat{P}_{a}\left(\tilde{\delta}_{\mathrm{opt}}^{(a)}\right)\right)$ and $\operatorname{MSE}\left(\hat{P}_{a}\left(\delta_{\mathrm{opt}}^{(a)}\right)\right)$ versus steering angle. True power uses the traditional definition as given in (3.27).
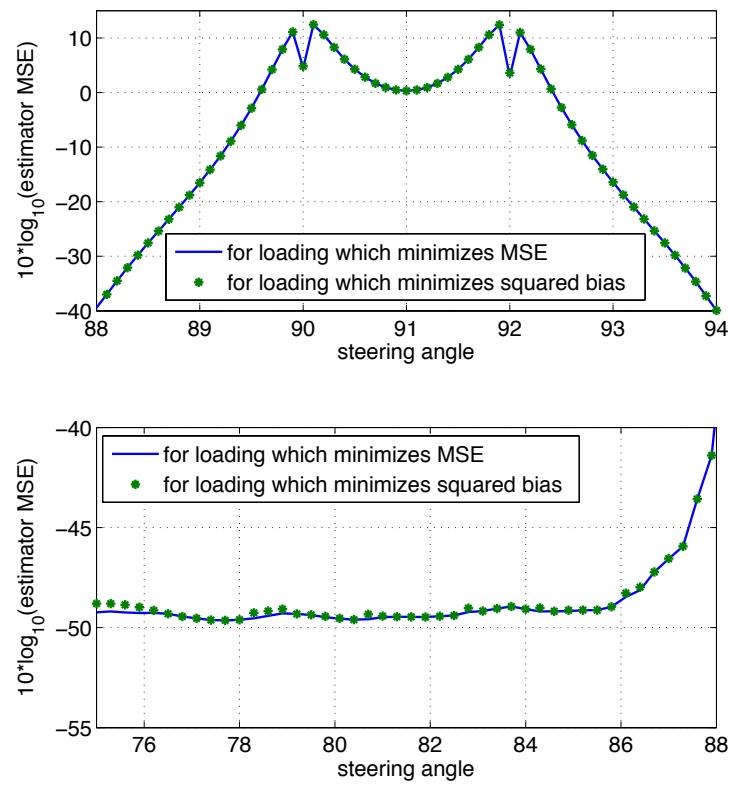

Figure 3-15: Scenario 1: $\operatorname{MSE}\left(\hat{P}_{b}\left(\tilde{\delta}_{\mathrm{opt}}^{(b)}\right)\right)$ and $\operatorname{MSE}\left(\hat{P}_{b}\left(\delta_{\mathrm{opt}}^{(b)}\right)\right)$ versus steering angle. True power uses the traditional definition as given in (3.27). 

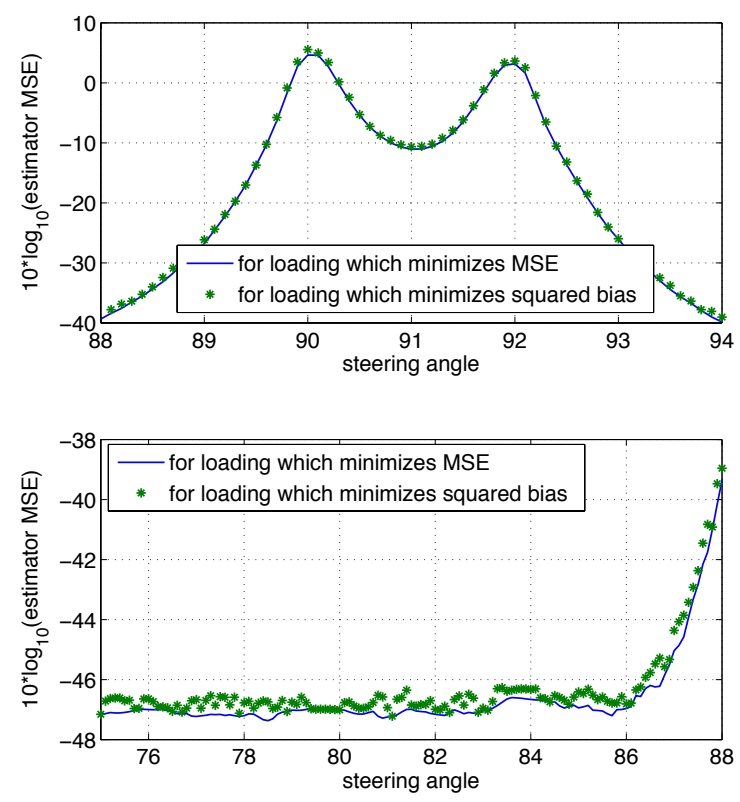

Figure 3-16: Scenario 1: $\operatorname{MSE}\left(\hat{P}_{a}\left(\tilde{\delta}_{\mathrm{opt}}^{(a)}\right)\right)$ and $\operatorname{MSE}\left(\hat{P}_{a}\left(\delta_{\mathrm{opt}}^{(a)}\right)\right)$ versus steering angle. True power uses the alternative definition as given in (3.28).
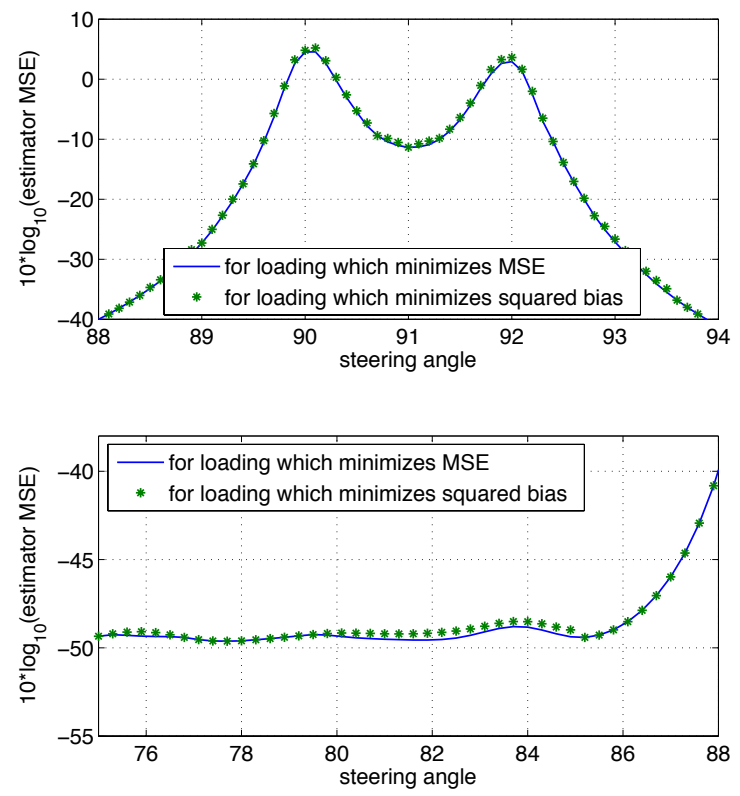

Figure 3-17: Scenario 1: $\operatorname{MSE}\left(\hat{P}_{b}\left(\tilde{\delta}_{\text {opt }}^{(b)}\right)\right)$ and $\operatorname{MSE}\left(\hat{P}_{b}\left(\delta_{\text {opt }}^{(b)}\right)\right)$ versus steering angle. True power uses the alternative definition as given in (3.28). 


\section{Scenario 2: Separated Arrivals with Low SNR in a Snapshot Poor Regime}

In this scenario, 2 signals are arriving at elevation angles of $90^{\circ}$ and $94^{\circ}$ with respect to the broadside of the array. Their SNR's are respectively $1 \mathrm{~dB}$ and $5 \mathrm{~dB}$. The array has 40 sensors and the number of snapshots available to estimate the SCM is 25. Note that $c=1.6$ and the normalized trace of the ensemble correlation matrix is 5.42 .

The simulated dependences of the squared bias, variance and MSE on diagonal loading for estimators $\hat{P}_{a}$ and $\hat{P}_{b}$, true powers evaluated using (3.27) and (3.28) and steering angle of $94.5^{\circ}$ are shown respectively in Figures 3-18 and 3-19. The same set of plots corresponding to steering angle of $87^{\circ}$ is shown in Figures $3-20$ and $3-21$. The plots confirm that the variance has almost no influence on a diagonal loading which minimizes the MSE.

Note that when steering close to the source direction, the MSE performance is optimized for non-zero loading, as shown in Fig 3-18. This is because the parameter $c=m / n$ is above 1 (i.e., the SCM is rank deficient) so that as $\delta \rightarrow 0$, the estimators $\hat{P}_{a}$ and $\hat{P}_{b}$ converge to 0 . Thus, as long as the true power is non-zero, the optimal diagonal loading is positive.

As in Scenario 1, a diagonal loading which optimizes the MSE corresponding to $\hat{P}_{b}$ does not exceed a loading which optimizes the MSE corresponding to $\hat{P}_{a}$. Also, the optimal loading pertaining to true power evaluated using (3.27) is smaller than the optimal loading pertaining to true power evaluated with (3.28) for both estimators.

The optimal MSE and the MSE evaluated at the minimizer $\tilde{\delta}_{\text {opt }}$ of the squared bias are compared for estimators $\hat{P}_{a}$ and $\hat{P}_{b}$ when the true power is evaluated with $(3.27)$ in Figures 3-22 and 3-23. The corresponding comparisons when the true power is evaluated with (3.28) are given in Figures 3-24 and 3-25.

As can be observed from the plots, the largest performance loss appears with estimator $\hat{P}_{a}$ when steering away from the main beams. However, this loss is still within $1 \mathrm{~dB}$. The performance loss corresponding to estimator $\hat{P}_{b}$ is negligible. The theoretical characterization of this loss is given in Section 3.7.2.

As a final remark, the optimized estimator $\hat{P}_{b}$ outperforms the optimized estimator 

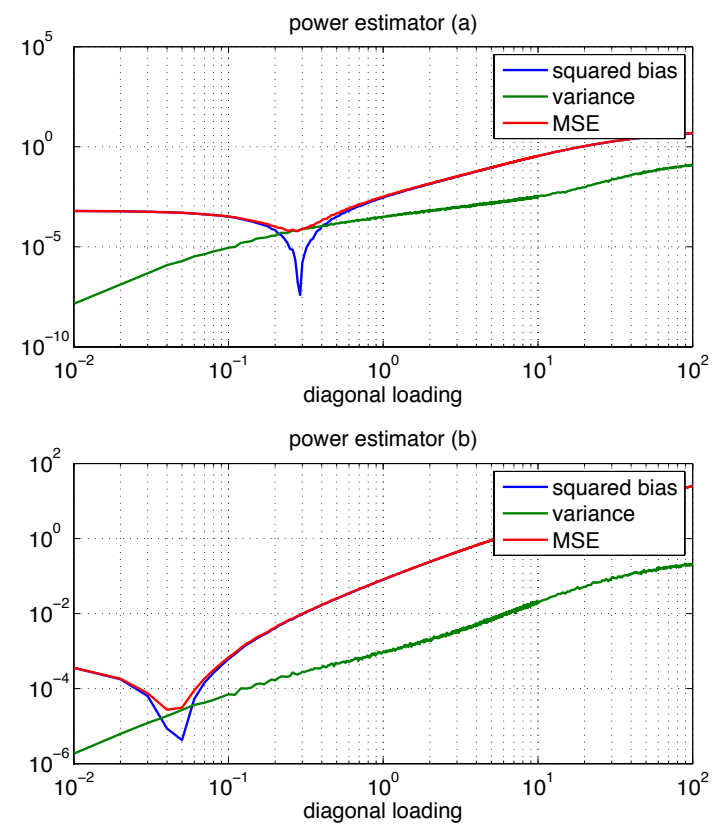

Figure 3-18: Scenario 2: Squared bias, variance and MSE corresponding to estimators $\hat{P}_{a}$ and $\hat{P}_{b}$ versus diagonal loading for steering angle of $94.5^{\circ}$. True power uses the traditional definition as given in (3.27).
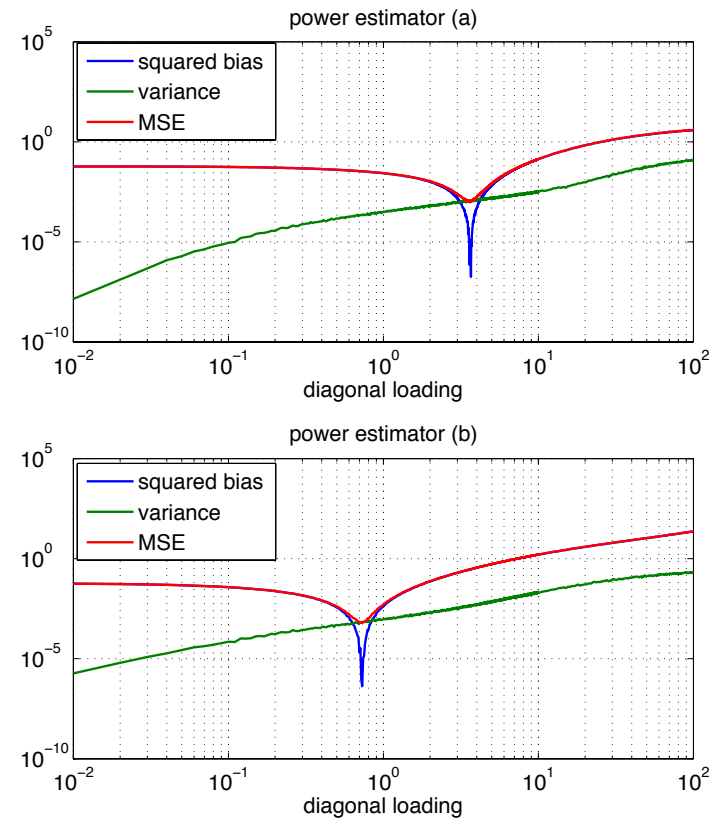

Figure 3-19: Scenario 2: Squared bias, variance and MSE corresponding to estimators $\hat{P}_{a}$ and $\hat{P}_{b}$ versus diagonal loading for steering angle of $94.5^{\circ}$. True power uses the alternative definition as given in (3.28). 

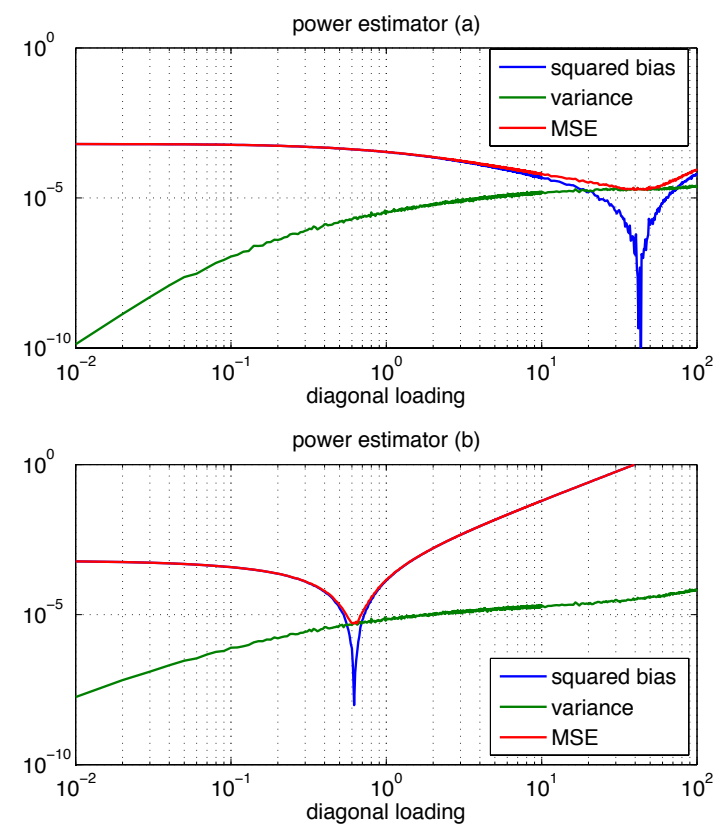

Figure 3-20: Scenario 2: Squared Bias, Variance and MSE corresponding to estimators $\hat{P}_{a}$ and $\hat{P}_{b}$ versus Diagonal Loading for Steering Angle of $87^{\circ}$. True power uses the traditional definition as given in (3.27).
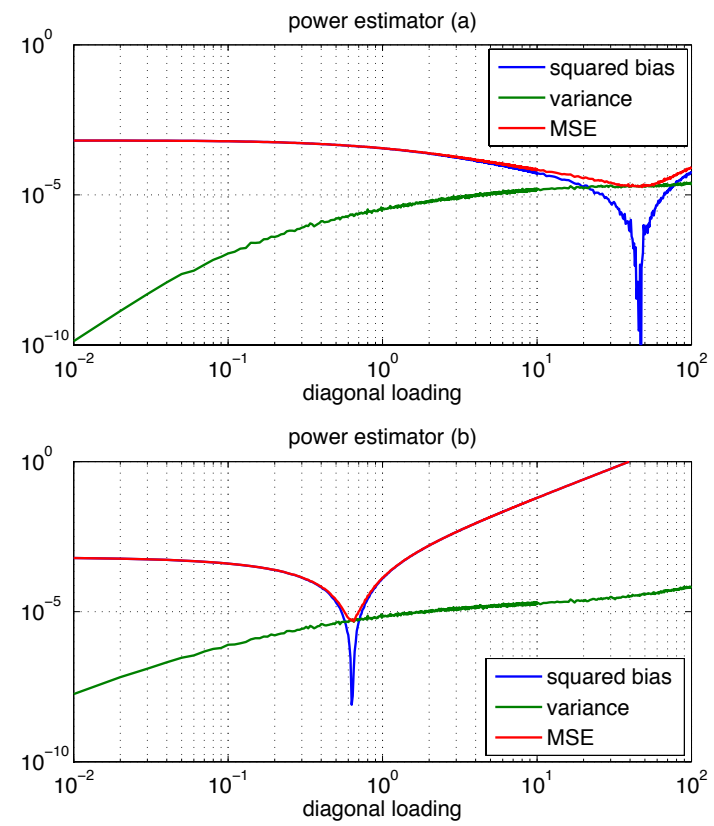

Figure 3-21: Scenario 2: Squared Bias, Variance and MSE corresponding to estimators $\hat{P}_{a}$ and $\hat{P}_{b}$ versus Diagonal Loading for Steering Angle of $87^{\circ}$. True power uses the alternative definition as given in (3.28). 

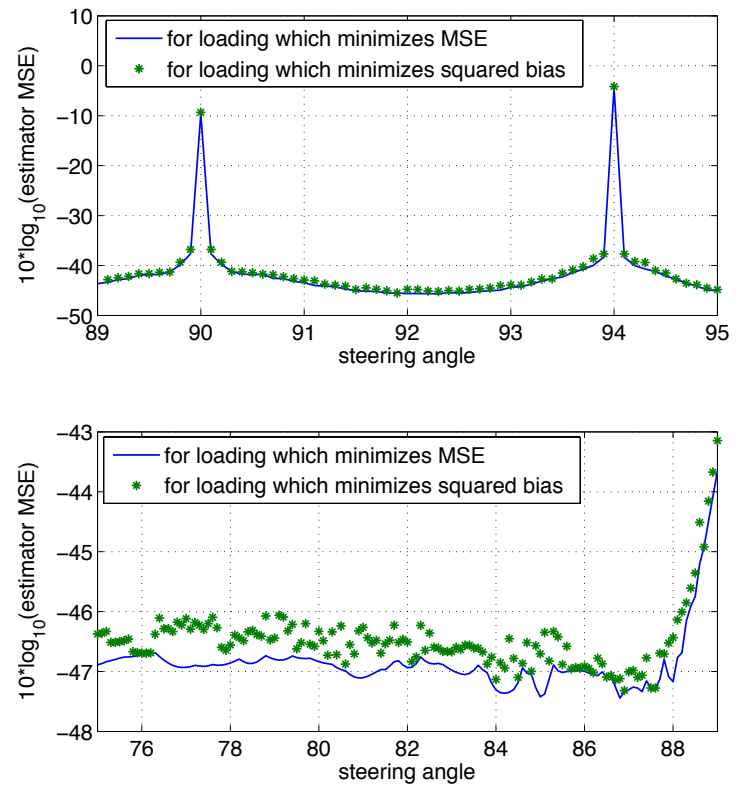

Figure 3-22: Scenario 2: $\operatorname{MSE}\left(\hat{P}_{a}\left(\tilde{\delta}_{\text {opt }}^{(a)}\right)\right)$ and $\operatorname{MSE}\left(\hat{P}_{a}\left(\delta_{\text {opt }}^{(a)}\right)\right)$ versus steering angle. True power uses the traditional definition as given in (3.27).

$\hat{P}_{a}$. The comparison between the estimators is treated more formally in Section 3.9.

\subsection{Behavior of Optimal Diagonal Loading}

The diagonal loading which minimizes the squared bias and approximately minimizes the estimation MSE is analyzed in this section and it is shown that the optimal diagonal loading depends on the steering direction. The analysis exploits the results from Section 3.4 pertaining to the behavior of the squared bias.

In general, for a given steering direction $\mathbf{v}_{s}$, the optimal loading $\tilde{\delta}_{\text {opt }}$ which minimizes the squared bias is for estimator $\hat{P}_{a}$

$$
\tilde{\delta}_{\text {opt }}^{(a)}\left\{\begin{array}{cc}
=0, \text { if } & P \leq \mathbf{E}\left[\hat{P}_{a}(0)\right] \\
\in(0, \infty), \text { if } & \mathbf{E}\left[\hat{P}_{a}(0)\right]<P<\lim _{\delta \rightarrow \infty} \mathbf{E}\left[\hat{P}_{a}(\delta)\right] \\
\rightarrow \infty, \text { if } & P \geq \lim _{\delta \rightarrow \infty} \mathbf{E}\left[\hat{P}_{a}(\delta)\right]
\end{array}\right.
$$



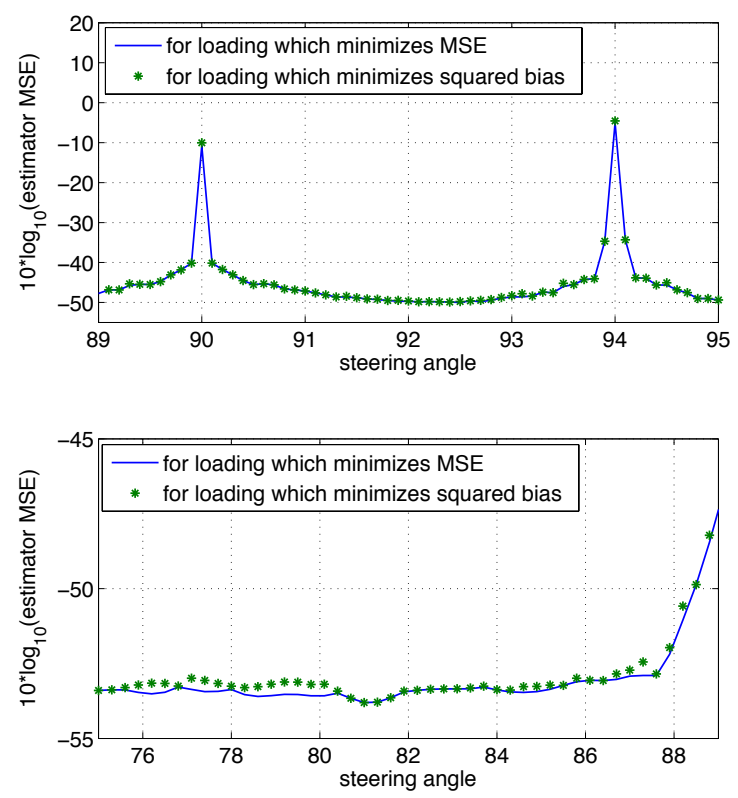

Figure 3-23: Scenario 2: $\operatorname{MSE}\left(\hat{P}_{b}\left(\tilde{\delta}_{\text {opt }}^{(b)}\right)\right)$ and $\operatorname{MSE}\left(\hat{P}_{b}\left(\delta_{\text {opt }}^{(b)}\right)\right)$ versus steering angle. True power uses the traditional definition as given in (3.27).
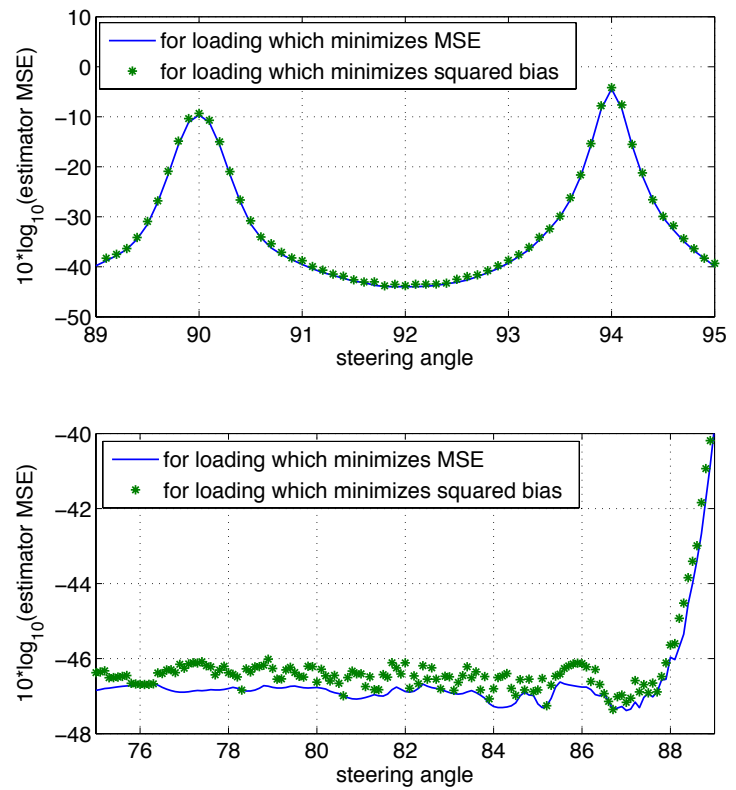

Figure 3-24: Scenario 2: $\operatorname{MSE}\left(\hat{P}_{a}\left(\tilde{\delta}_{\text {opt }}^{(a)}\right)\right)$ and $\operatorname{MSE}\left(\hat{P}_{a}\left(\delta_{\text {opt }}^{(a)}\right)\right)$ versus steering angle. True power uses the alternative definition as given in (3.28). 

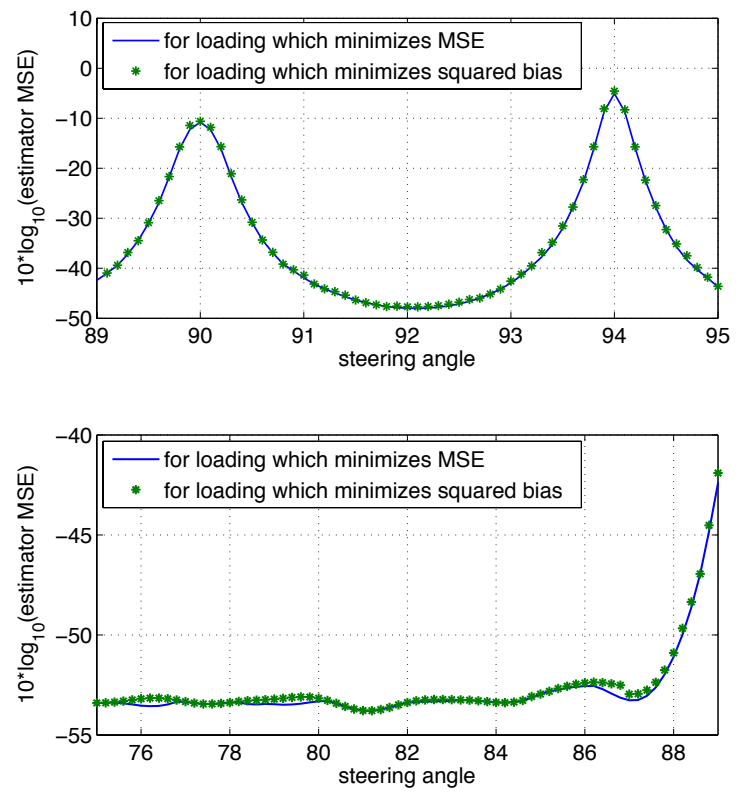

Figure 3-25: Scenario 2: $\operatorname{MSE}\left(\hat{P}_{b}\left(\tilde{\delta}_{\text {opt }}^{(b)}\right)\right)$ and $\operatorname{MSE}\left(\hat{P}_{b}\left(\delta_{\text {opt }}^{(b)}\right)\right)$ versus steering angle. True power uses the alternative definition as given in (3.27).

and for estimator $\hat{P}_{b}$,

$$
\tilde{\delta}_{\text {opt }}^{(b)}\left\{\begin{array}{ccc}
=0, & \text { if } & P \leq \mathbf{E}\left[\hat{P}_{b}(0)\right] \\
\in(0, \infty), & \text { if } & P>\mathbf{E}\left[\hat{P}_{b}(0)\right]
\end{array}\right.
$$

where $P$ is the true power in the direction described by $\mathbf{v}_{s}$ with respect to which the MSE of a power estimator is optimized. The limiting expectations $\mathbf{E}[\hat{P}(0)]$ and $\lim _{\delta \rightarrow \infty} \mathbf{E}[\hat{P}(\delta)]$ are evaluated using the results of Lemma 3.2.

In addition, invoking that $\hat{P}_{b}(\delta) \geq \hat{P}_{a}(\delta)$, we immediately conclude that for a given steering direction $\mathbf{v}_{s}$ and the true power $P$ associated with it,

$$
\tilde{\delta}_{\mathrm{opt}}^{(b)} \leq \tilde{\delta}_{\mathrm{opt}}^{(a)}
$$

with equality for $P \leq \mathbf{E}[\hat{P}(0)]$ (in which case, these loadings are equal to zero).

In the following parts, arrival models consisting of a single and multiple plane waves embedded into spatially uncorrelated noise (i.e., the noise is isotropic and the 
Table 3.1: Expected Power Estimators in the Limit

\begin{tabular}{|c|c|c|}
\hline & $\mathbf{v}_{s}=\mathbf{v}_{o}$ & $\mathbf{v}_{s} \neq \mathbf{v}_{o}$ \\
\hline $\lim _{\delta \rightarrow \infty} \mathbf{E}\left[\hat{P}_{a}(\delta)\right]=$ & $P_{o}+\frac{\sigma_{v}^{2}}{m}$ & $P_{o} \frac{\left|\mathbf{v s}_{s}^{H} \mathbf{v}_{o}\right|^{2}}{m^{2}}+\frac{\sigma_{v}^{2}}{m}$ \\
\hline $\mathbf{E}\left[\hat{P}^{a}(0)\right]=\mathbf{E}\left[\hat{P}^{b}(0)\right] \approx$ & $\left(1-\frac{m}{n}\right)\left(P_{o}+\frac{\sigma_{v}^{2}}{m}\right)$ & $\frac{\sigma_{v}^{2}\left(1-\frac{m}{n}\right)\left(\sigma_{v}^{2}+m P_{o}\right)}{m\left(\sigma_{v}^{2}+m P_{o}\right)-P_{o}\left|\mathbf{v}_{s}^{H} \mathbf{v}_{o}\right|^{2}}$ \\
\hline
\end{tabular}

sensors are separated half-a-wavelength) are considered. The behavior of the optimal diagonal loading is analyzed with respect to the standard definition of true power in the first part of this section. The analysis corresponding to the alternative definition of true power is given in the second part. The last part validates the obtained results via simulations.

\subsubsection{Single Source in Uncorrelated Noise}

An ensemble correlation matrix $\mathbf{R}$ of the received snapshots originating from a single source transmitting a signal of power $P_{o}$ in the direction described by $\mathbf{v}_{o}$ and embedded into an uncorrelated noise of variance $\sigma_{v}^{2}$ is

$$
\mathbf{R}=P_{o} \mathbf{v}_{o}^{H} \mathbf{v}_{o}+\sigma_{v}^{2} \mathbf{I} .
$$

Using the matrix inversion lemma, the inverse of the spatial correlation matrix can be written as

$$
\mathbf{R}^{-1}=\frac{1}{\sigma_{v}^{2}}\left(\mathbf{I}-\frac{P_{o} \mathbf{v}_{o}^{H} \mathbf{v}_{o}}{\sigma_{v}^{2}+m P_{o}}\right) .
$$

Substituting (3.113) and (3.114) into the expressions of Lemma 3.2, the results concerning the limiting behavior of the power estimators for $\delta=0$ and $\delta \rightarrow \infty$ when steering in and off the source direction $\mathbf{v}_{o}$ and when $n>m$ are obtained and summarized in Table 3.1. Recall that the power estimator $\hat{P}_{b}$ is unbounded when $\delta \rightarrow \infty$ and this case is not shown in the table. Note that when $m>n$ and $\delta=0$, the SCM has zero eigenvalues so that the power estimate in the steering direction becomes zero unless some sort of subspace processing is employed. 
The true power is using (3.27) given by

$$
P\left(\mathbf{v}_{s}\right)=\left\{\begin{array}{c}
\frac{\sigma_{v}^{2}}{m}, \text { if } \mathbf{v}_{s} \neq \mathbf{v}_{o} \\
P_{o}+\frac{\sigma_{v}^{2}}{m}, \text { if } \mathbf{v}_{s}=\mathbf{v}_{o}
\end{array}\right.
$$

From the results in Table 3.1, true power definition (3.115) and characterizations (3.110) and (3.111), we deduce some facts about optimal loading $\tilde{\delta}_{\text {opt }}$. Namely, when steering in the source direction such that $\mathbf{v}_{s}=\mathbf{v}_{o}$, the squared bias pertaining to $\hat{P}_{a}$ is minimized (canceled out) when $\delta \rightarrow \infty$, i.e., the optimal processor in this case is the matched filter. Since $\hat{P}_{b}(\delta)$ is unbounded when $\delta \rightarrow \infty$, the optimal loading $\tilde{\delta}_{\text {opt }}^{(b)}$ is always finite.

On the other hand, when steering off the source direction such that $\mathbf{v}_{s} \neq \mathbf{v}_{o}$, the optimal loading $\tilde{\delta}_{\text {opt }}$ depends on the value of the inner product $\mathbf{v}_{s}^{H} \mathbf{v}_{o}$. Namely, from (3.110) and (3.111), the squared bias corresponding to both $\hat{P}_{a}$ and $\hat{P}_{b}$ is minimized for zero loading when $P=\frac{\sigma_{v}^{2}}{m} \leq \mathbf{E}[\hat{P}(0)]$. This condition is in the $n>m$ regime using the result from Table 3.1 equivalent to

$$
\left|\frac{\mathbf{v}_{s}^{H} \mathbf{v}_{o}}{m}\right|^{2} \geq \frac{m}{n}\left(1+\frac{\sigma_{v}^{2}}{m P_{o}}\right) .
$$

For the case when $\mathbf{v}_{s}$ and $\mathbf{v}_{o}$ are orthogonal, then the true power $P=\frac{\sigma_{v}^{2}}{m}$ coincides with $\lim _{\delta \rightarrow \infty} \mathbf{E}\left[\hat{P}_{a}(\delta)\right]$ and consequently, $\tilde{\delta}_{\mathrm{opt}}^{(a)}=\infty$. In other words, when $\mathbf{v}_{s}^{H} \mathbf{v}_{o}=0$, the matched filter completely removes the interference originating from the source meaning that it is the optimal processor. In addition, as $\mathbf{v}_{s}$ moves away from $\mathbf{v}_{o}$, the inner product $\left|\mathbf{v}_{s}^{H} \mathbf{v}_{s}\right|$ tends to decrease such that $\lim _{\delta \rightarrow \infty} \mathbf{E}\left[\hat{P}_{a}(\delta)\right]$ approaches the level of the true power $P$. Hence, the optimal loading $\tilde{\delta}_{\text {opt }}^{(a)}$ tends to increase. The behavior of the optimal loading $\tilde{\delta}_{\text {opt }}^{(b)}$ corresponding to $\hat{P}_{b}$ exhibits similar trends, with the distinction that it is always finite.

The preceding observations are summarized in Table 3.2. A graphical visualization of the results is given in Fig. 3-26, where the true power $P$ and the expected values of the estimator $\hat{P}_{a}$ when $\delta=0$ and $\delta \rightarrow \infty$ for a range of steering angles, are shown. The specific scenario used to generate Fig. 3-26 is described in its caption. A value 
Table 3.2: Properties of $\tilde{\delta}_{\text {opt }}$

\begin{tabular}{|c|c|c|}
\hline & $\mathbf{v}_{s}=\mathbf{v}_{o}$ & $\mathbf{v}_{s} \neq \mathbf{v}_{o}$ \\
\hline$\tilde{\delta}_{\text {opt }}^{(a)}$ & $\infty$ & $\begin{array}{c}\infty, \text { if } \mathbf{v}_{s}^{H} \mathbf{v}_{o}=0 \\
0, \text { if condition }(3.116) \\
\in(0, \infty), \text { otherwise }\end{array}$ \\
\hline$\tilde{\delta}_{\text {opt }}^{(b)}$ & finite & $\begin{array}{c}0, \text { if condition }(3.116) \\
\in(0, \infty), \text { otherwise }\end{array}$ \\
\hline
\end{tabular}

of $\mathbf{E}\left[\hat{P}_{a}(\delta)\right]$ is always between $\mathbf{E}[\hat{P}(0)]$ and $\mathbf{E}\left[\hat{P}_{a}(\infty)\right]$ because $\hat{P}_{a}$ monotonically increases. For a given steering angle and if $P$ is above $\mathbf{E}[\hat{P}(0)]$, as $\delta$ changes from 0 to $\infty$, the expected estimate moves from the $\mathbf{E}\left[\hat{P}_{a}(0)\right]$ curve, intersects a curve representing the true power $P$ and asymptotically approaches $\mathbf{E}\left[\hat{P}_{a}(\infty)\right]$ curve. As a rule of thumb, whenever the true power $P$ gets close to $\mathbf{E}\left[\hat{P}_{a}(\infty)\right]$, the optimal loading $\tilde{\delta}_{\text {opt }}^{(a)}$ becomes large. When $P$ is below $\mathbf{E}\left[\hat{P}_{a}(0)\right]$, the squared bias is minimized for $\delta=0$. A graphical representation corresponding to the estimator $\hat{P}_{b}$ is similar, with the difference that $\mathbf{E}\left[\hat{P}_{b}\right]$ blows up as $\delta \rightarrow \infty$ such that $\tilde{\delta}_{\text {opt }}^{(b)}$ is always finite.

The dependance of optimal loading $\tilde{\delta}_{\text {opt }}^{(a)}$ on steering angle is shown in Fig. 3-27. As expected from Fig. 3-26, the optimal loading oscillates and increases as the steering angle moves away from the source direction. The optimal loading is clipped at 200. In contrast, the optimal loading $\tilde{\delta}_{\text {opt }}^{(b)}$ for the considered example is 15.5 in the source direction and does not exceed the value of 0.38 when steering off the source direction. This happens because $\hat{P}_{b}$ is much larger than $\hat{P}_{a}$ when $\delta$ is moderately greater than zero such that $\mathbf{E}\left[\hat{P}_{b}\right]$ intersects the level of $P$ much sooner than $\mathbf{E}\left[\hat{P}_{a}\right]$ does.

\subsubsection{Multiple Sources in Uncorrelated Noise}

Now we consider a case of a number of sources $M$, each transmitting a signal of power $P_{i}$ in the direction specified by $\mathbf{v}_{i}$ such that the ensemble correlation matrix of the received snapshots is ${ }^{6}$

$$
\mathbf{R}=\sum_{k=1}^{M} P_{i} \mathbf{v}_{i}^{H} \mathbf{v}_{i}+\sigma^{2} \mathbf{I}
$$

\footnotetext{
${ }^{6}$ This model is called separable souces in [52].
} 


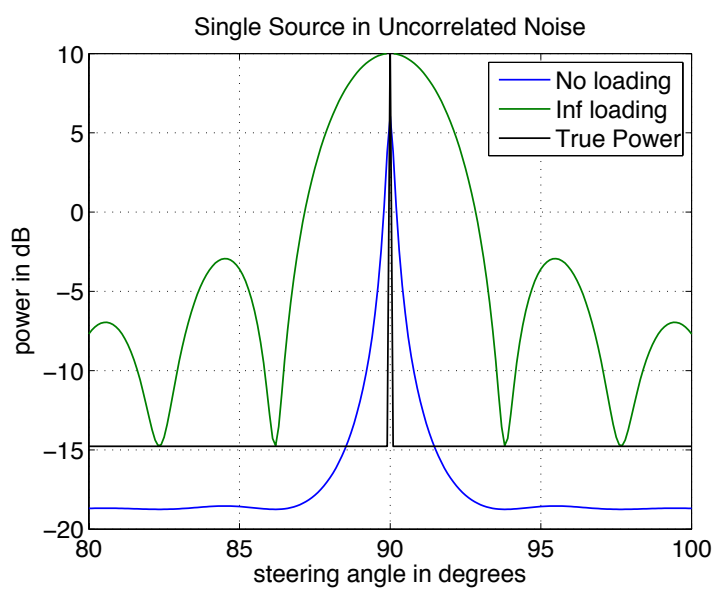

Figure 3-26: True power according to (3.115), $\mathbf{E}\left[\hat{P}_{a}(0)\right]$ and $\mathbf{E}\left[\hat{P}_{a}(\infty)\right]$. A signal has power 10 and arrives from $90^{\circ}$. Noise is uncorrelated and its variance is 1 . The array has 30 sensors and 50 snapshots are available.
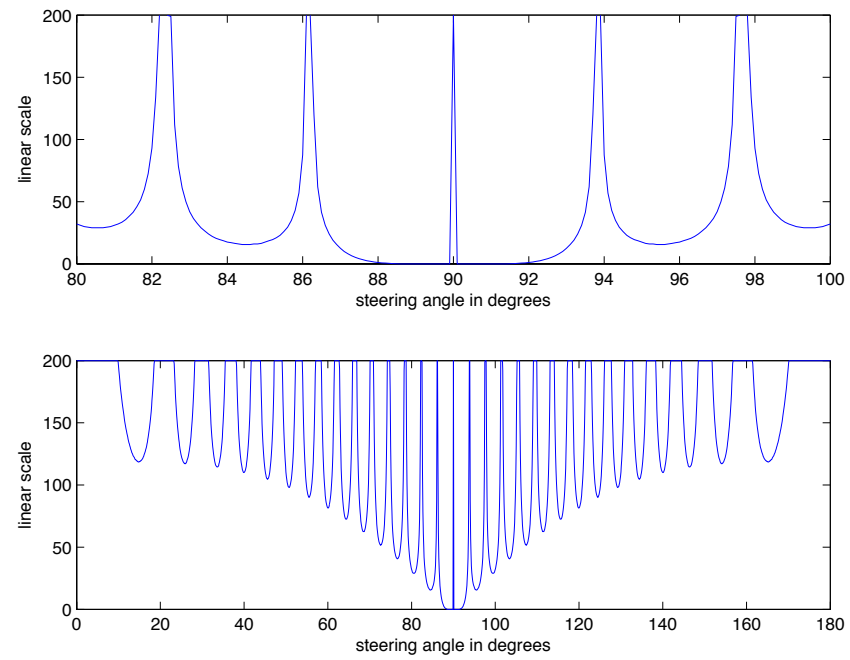

Figure 3-27: Optimal loading $\tilde{\delta}_{\text {opt }}^{(a)}$. A signal is of power 10 and arrives from $90^{\circ}$. Noise is uncorrelated, of power 1. A diagonal loading is clipped at 200. Whenever the steering and source directions are the same or orthogonal, $\tilde{\delta}_{\text {opt }}^{(a)}=\infty$. 
where $\sigma^{2}$ is the variance of the uncorrelated noise.

The limiting expectation of the estimator $\hat{P}_{a}$ when the steering direction $\mathbf{v}_{s} \neq \mathbf{v}_{i}$, $i=1, \ldots, M$, is using Lemma 3.2 given by

$$
\lim _{\delta \rightarrow \infty} \mathbf{E}\left[\hat{P}_{a}(\delta)\right]=\sum_{k=1}^{M} P_{i}\left|\frac{\mathbf{v}_{s}^{H} \mathbf{v}_{i}}{m}\right|^{2}+\frac{\sigma_{v}^{2}}{m} .
$$

Therefore, since in general, $\lim _{\delta \rightarrow \infty} \mathbf{E}\left[\hat{P}_{a}(\delta)\right]>P=\frac{\sigma_{v}^{2}}{m}$, the optimal loading $\tilde{\delta}_{\mathrm{opt}}^{(a)}$ is finite. In a special case when $\mathbf{v}_{s}$ is orthogonal with each of $\mathbf{v}_{i}, i=1, \ldots, M$, the optimal beamformer is a matched filter.

Similarly, when steering in a source direction,

$$
\lim _{\delta \rightarrow \infty} \mathbf{E}\left[\hat{P}_{a}(\delta)\right]=P_{1}+\sum_{k=2}^{M} P_{i}\left|\frac{\mathbf{v}_{s}^{H} \mathbf{v}_{i}}{m}\right|^{2}+\frac{\sigma_{v}^{2}}{m}
$$

where without loss of generality $\mathbf{v}_{s}=\mathbf{v}_{1}$. Again, in general, $\lim _{\delta \rightarrow \infty} \mathbf{E}\left[\hat{P}_{a}(\delta)\right]>P=$ $P_{1}+\frac{\sigma_{v}^{2}}{m}$ so that the optimal loading is finite. In a special case when $\mathbf{v}_{s}^{H} \mathbf{v}_{i}=0$ for $i=2, \ldots, M$, the optimal estimator is a matched filter.

Certainly, when steering slightly away from the source direction, the true power $P=\frac{\sigma_{v}^{2}}{m}$ is below the smallest expected estimate achieved for $\delta=0$. The squared bias is minimized for $\delta=0$ in such a case. A general characterization of the steering directions for which $\tilde{\delta}_{\text {opt }}^{(a)}=0$ is cumbersome due to lack of compact expression for $\mathbf{R}^{-1}$. Therefore, we need to resort to a specific assignment of the arriving directions $\mathbf{v}_{i}$ and powers $P_{i}$.

A graphical visualization of the stated results is given in Fig. 3-28 where two arrivals have the same power and are closely spaced. A similar discussion as given for a single source case applies here as well. A plot of optimal loading $\tilde{\delta}_{\text {opt }}^{(a)}$ is shown in Fig. 3-29. Again, when non-zero, the optimal loading $\tilde{\delta}_{\text {opt }}^{(b)}$ is much smaller than $\tilde{\delta}_{\text {opt }}^{(b)}$ and does not exceed the value of 0.38 when steering off the main beams. 


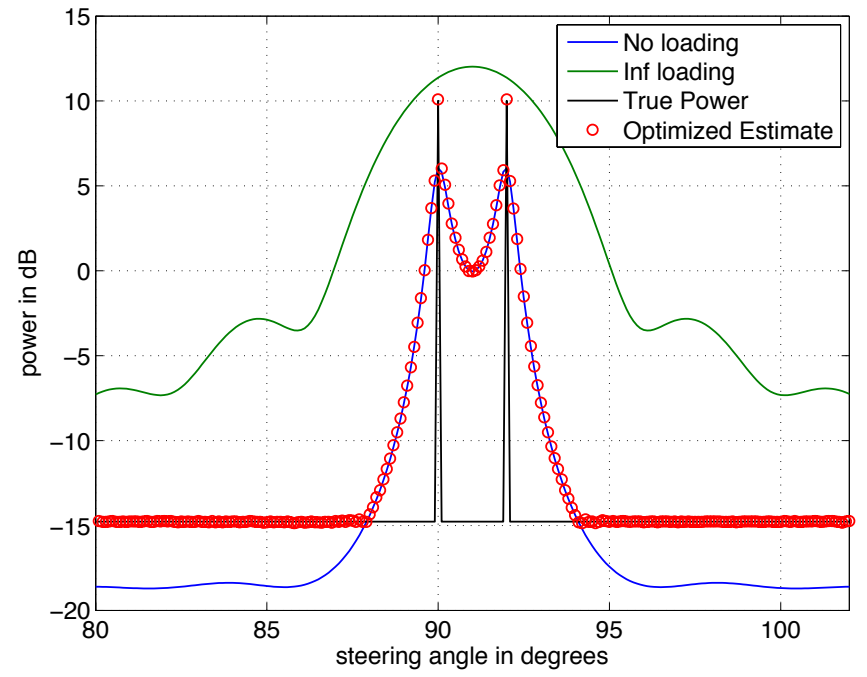

Figure 3-28: True power according to (3.27), $\mathbf{E}\left[\hat{P}_{a}(0)\right], \mathbf{E}\left[\hat{P}_{a}(\infty)\right]$ and the expected optimized power estimate obtained via Monte-Carlo simulations. Scenario 1 is considered, namely, each of two plane waves has power 10 and they arrive at $90^{\circ}$ and $92^{\circ}$ to the broadside. The noise is uncorrelated and its variance is 1 . The array is linear, $u / 2$ spaced and has 30 sensors. 50 snapshots are available.

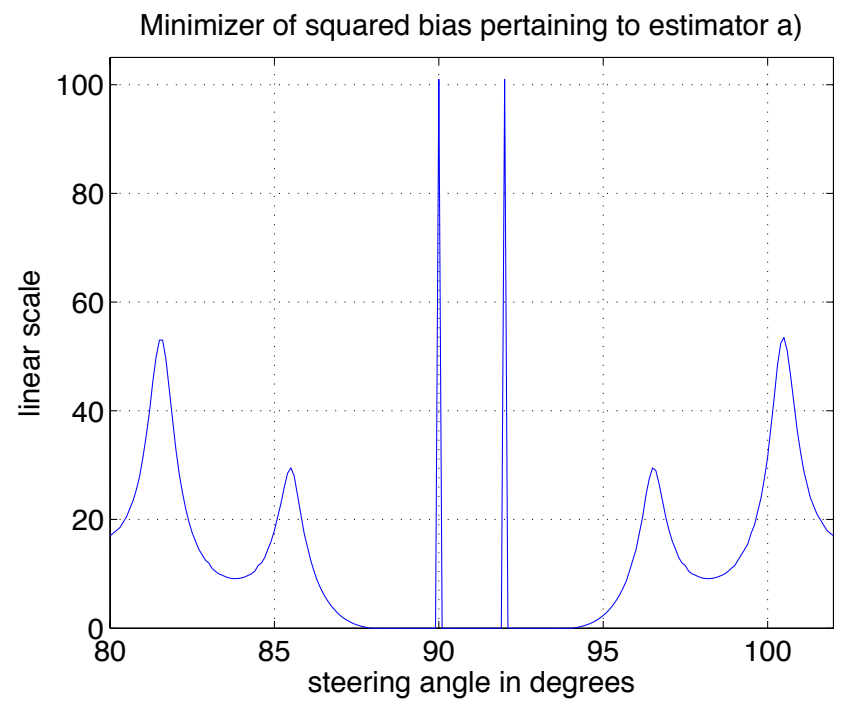

Figure 3-29: Optimal loading $\tilde{\delta}_{\text {opt }}^{(a)}$ for Scenario 1. 


\subsubsection{Alternative Definition of True Power}

In the example of a single source in uncorrelated noise, it can be shown that if the array is steered in the source direction, then the power $P$ as defined in (3.28) is

$$
P=\lim _{\delta \rightarrow \infty} \hat{P}_{a}(\delta)
$$

i.e., the optimal loading $\tilde{\delta}_{\mathrm{opt}}^{(a)} \rightarrow \infty$. In comparison, the squared bias corresponding to $\hat{P}_{b}$ is always minimized for finite loading $\tilde{\delta}_{\mathrm{opt}}^{(b)}$.

A more interesting fact is to observe using Lemma 3.2 that

$$
\mathbf{E}[\hat{P}(0)] \approx \frac{1-c}{\mathbf{v}_{s}^{H} \mathbf{R}^{-1} \mathbf{v}_{s}} \leq P
$$

where $c<1$ is implicitly assumed by considering the unloaded estimator.

This means that unless $c=0$, when no loading is needed, the optimal loading $\tilde{\delta}_{\text {opt }}$ is positive. This is graphically shown in Fig. 3-30, which corresponds to the same

scenario as in Fig. 3-28. Since the true power is always greater than $\mathbf{E}\left[\hat{P}_{a}(0)\right]$, the optimal loading $\tilde{\delta}_{\mathrm{opt}}^{(a)}$ is always non-zero.

Note that when steering away from the source direction, the powers defined in (3.27) and (3.28) converge such that the associated optimal loadings coincide. On the contrary, when steering close to the source direction, diagonal loadings optimized with respect to two definitions significantly differ.

\subsubsection{Numerical Validation}

This section validates the results which describe behavior of optimal diagonal loading when the arrival process is composed of plane waves. A standard, vertical, linear array with $u / 2$ separation of the elements is considered. In addition, a spatially uncorrelated, zero-mean noise with a variance of one corrupts the signal snapshots. Two different scenarios are considered. 


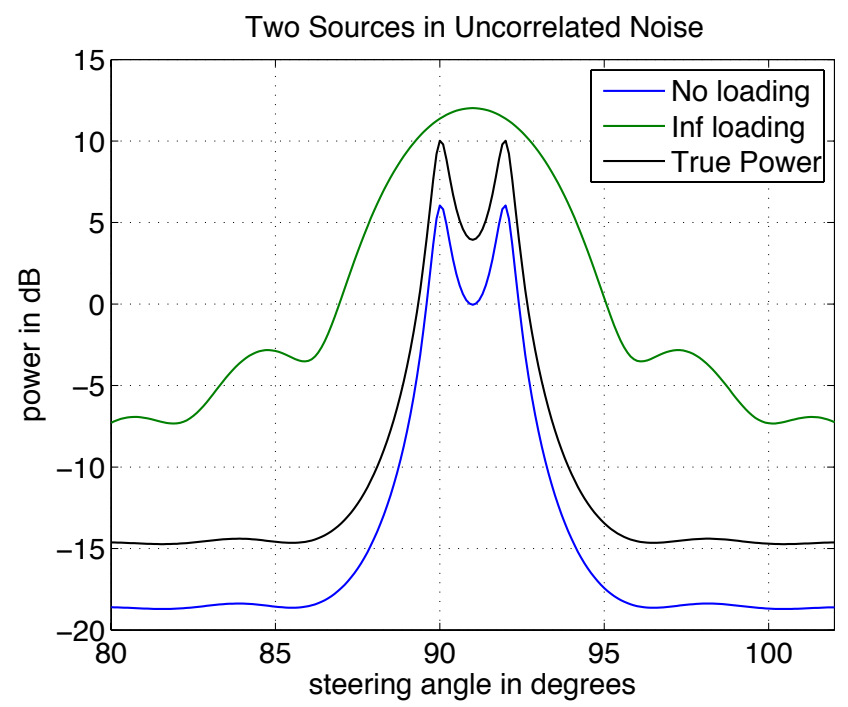

Figure 3-30: True power according to (3.28), $\mathbf{E}\left[\hat{P}_{a}(0)\right]$ and $\mathbf{E}\left[\hat{P}_{a}(\infty)\right]$ for Scenario 1

\section{Scenario 1}

In this scenario, 2 signals are arriving at elevation angles of $90^{\circ}$ and $92^{\circ}$ and each has power 10 (i.e., the SNR is $10 \mathrm{~dB}$ ). The array contains 30 sensors and 50 snapshots are used to estimate the sample correlation matrix.

To validate the theoretical findings in this section, the simulated optimal diagonal loadings versus steering angle are plotted in Fig. 3-31 for true power evaluated using a standard approach (3.27). As shown, there is a range of steering directions for which a nearly diagonally unloaded estimator achieves the lowest MSE. The smallest diagonal loading used in the simulation tests is $10^{-2}$ and is optimal when steering close to main beams. In addition, the estimator $\hat{P}_{b}$ is optimized at smaller values of diagonal loading which do not vary significantly across the steering directions.

The plots of simulated optimal diagonal loadings versus steering angle for true power evaluated using the alternative approach (3.28) are shown in Fig. 3-32. As theoretically elaborated in previous parts, when an alternative definition of true power is used, the performance is optimized for positive loadings. 

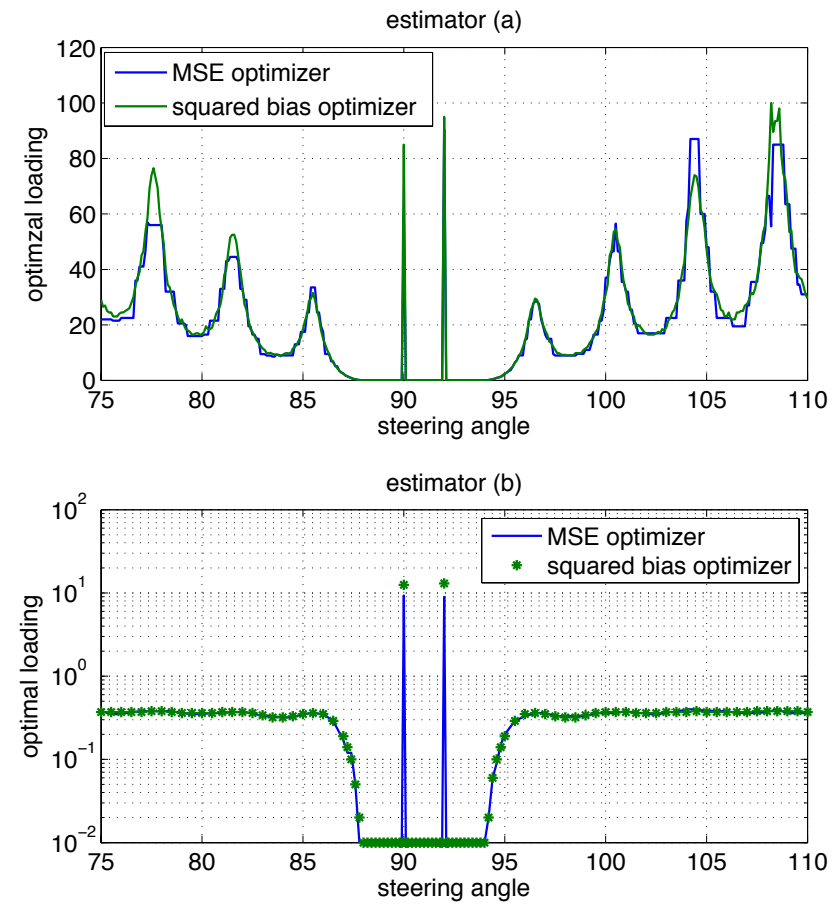

Figure 3-31: Scenario 1: $\delta_{\text {opt }}$ and $\tilde{\delta}_{\text {opt }}$ versus steering angle for $\hat{P}_{a}$ and $\hat{P}_{b}$. True power uses the traditional definition as given in (3.27).

\section{Scenario 2}

In this scenario, 2 signals are arriving at elevation angles of $90^{\circ}$ and $94^{\circ}$ with respect to the broadside of the array. Their SNR's are respectively $1 \mathrm{~dB}$ and $5 \mathrm{~dB}$. The array has 40 sensors and the number of snapshots available to estimate the SCM is 25 .

The plots of the simulated optimal diagonal loading versus steering angle for true powers evaluated using both the standard and alternative approaches are respectively shown in Figures 3-33 and 3-34. As expected, the optimal diagonal loading is always positive because in this scenario, $c>1$ (i.e., the SCM is rank deficient). However, even though the SCM is rank deficient in this case, the optimal diagonal loading when steering close to source directions is small. This is in accordance with our earlier observation that when steering close to main beams, the optimal estimator tends to perform adaptation as much as possible, fully relying on the available data.

As a rule of thumb, it has been observed that the sensitivities of the MSE and squared bias around their minimizers decrease as $\delta_{\text {opt }}$ and $\tilde{\delta}_{\text {opt }}$ get large. This effect, 

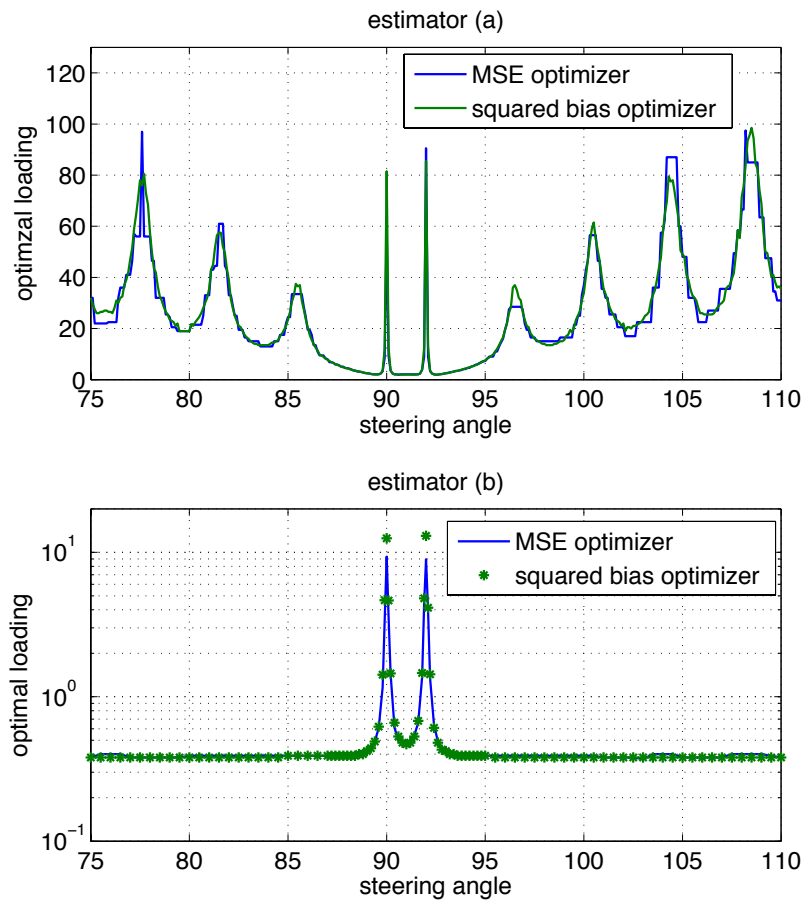

Figure 3-32: Scenario 1: $\delta_{\text {opt }}$ and $\tilde{\delta}_{\text {opt }}$ versus steering angle for $\hat{P}_{a}$ and $\hat{P}_{b}$. True power uses the alternative definition as given in (3.28).

together with the variability in the simulated curves, causes a disagreement in the peaks of the simulated $\delta_{\text {opt }}^{(a)}$ and $\tilde{\delta}_{\text {opt }}^{(a)}$. In addition, this likely contributes to the performance loss observed in the simulations (such as in Figures 3-16 and 3-14) when steering away from the sources. As an illustration, the dependence of the squared bias, variance and MSE corresponding to power estimator $\hat{P}_{a}$ on diagonal loading when steering in the direction of $76^{\circ}$ is shown in Fig. 3-35.

\subsection{Comparison between Power Estimators}

The optimized mean square errors and sensitivities to optimal diagonal loading of the power estimators $\hat{P}_{a}$ and $\hat{P}_{b}$ are compared in this section. The theoretical result concerning the estimation performance is stated and proved in the first part. The sensitivities of the power estimators on optimal diagonal loading are compared in the second part. The simulation results presented in the last part validate the theoretical 

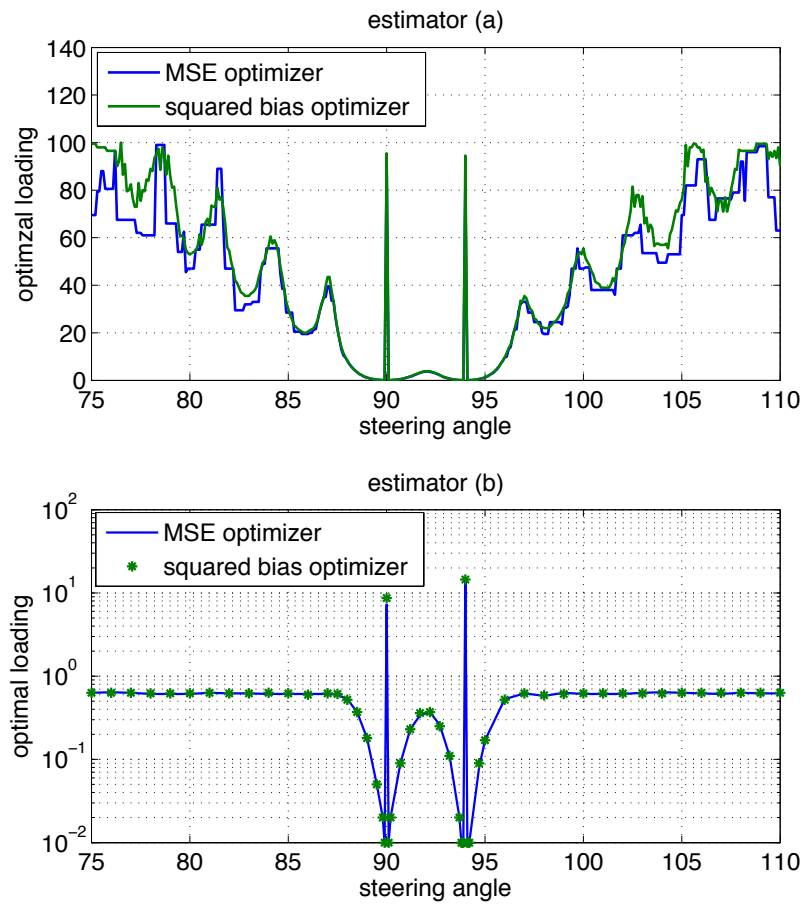

Figure 3-33: Scenario 2: $\delta_{\text {opt }}$ and $\tilde{\delta}_{\text {opt }}$ versus steering angle for $\hat{P}_{a}$ and $\hat{P}_{b}$. True power uses the traditional definition as given in (3.27).

finding.

\subsubsection{Comparison based on Estimation Performance}

In comparing the MSE performance of power estimators, we rely on the conjecture that the difference between the MSE's evaluated at optimal loading $\delta_{\text {opt }}$ and the loading $\tilde{\delta}_{\text {opt }}$ which minimizes the squared bias is negligible for both estimators. Therefore, we first compare the MSE's of the two estimators evaluated at respectively $\tilde{\delta}_{\text {opt }}^{(a)}$ and $\tilde{\delta}_{\text {opt }}^{(b)}$.

The following result establishes that estimator $\hat{P}_{a}$ has larger variance than the estimator $\hat{P}_{b}$ when both variances are measured at loadings which minimize the corresponding squared biases.

Lemma 3.7. Denoting by $\tilde{\delta}_{\text {opt }}^{(a)}$ and $\tilde{\delta}_{\text {opt }}^{(b)}$ the loadings which minimize the squared biases of respectively $\hat{P}_{a}$ and $\hat{P}_{b}$ for some steering direction described by $\mathbf{v}_{s}$ and true power 

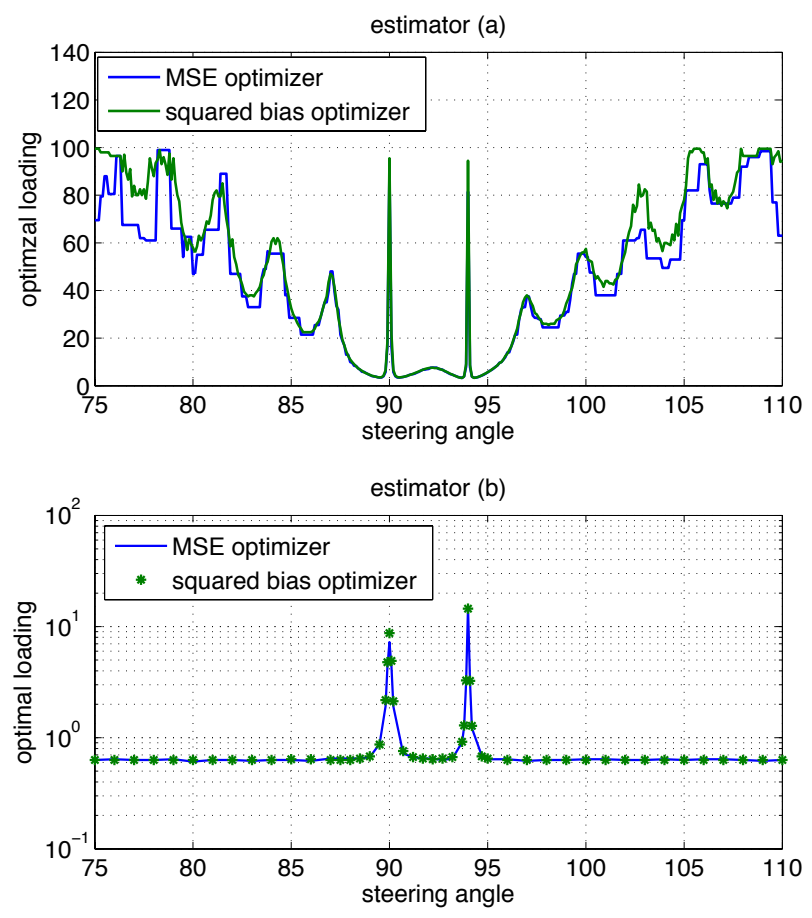

Figure 3-34: Scenario 2: $\delta_{\text {opt }}$ and $\tilde{\delta}_{\text {opt }}$ versus steering angle for $\hat{P}_{a}$ and $\hat{P}_{b}$. True power uses the alternative definition as given in (3.28).

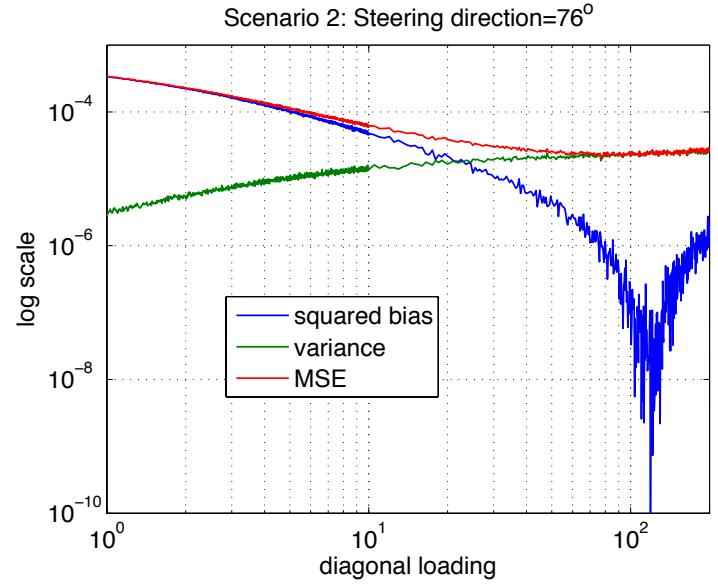

Figure 3-35: Scenario 2: Squared bias, variance and MSE for $\hat{P}_{a}$ versus diagonal loading for steering angle of $76^{\circ}$. 
$P \leq \lim _{\delta \rightarrow \infty} \mathbf{E}\left[\hat{P}_{a}(\delta)\right]$, the corresponding variances are related as

$$
\operatorname{var}\left(\hat{P}_{a}\left(\tilde{\delta}_{o p t}^{(a)}\right)\right) \geq \operatorname{var}\left(\hat{P}_{b}\left(\tilde{\delta}_{o p t}^{(b)}\right)\right)
$$

Proof. In a trivial case when $P \leq \mathbf{E}[\hat{P}(0)]$, the two biases are minimized for $\delta=0$ so that (3.122) follows directly with the equality sign.

A slightly more involved case arises when $P>\mathbf{E}[\hat{P}(0)]$. To simplify the exposition, the notation $Q_{k, a}=Q_{k}\left(\tilde{\delta}_{\mathrm{opt}}^{(a)}\right)$ and $Q_{k, b}=Q_{k}\left(\tilde{\delta}_{\mathrm{opt}}^{(b)}\right)$ is introduced.

Due to assumption $P \leq \lim _{\delta \rightarrow \infty} \mathbf{E}\left[\hat{P}_{a}(\delta)\right]$, it follows that $\tilde{\delta}_{\text {opt }}^{(a)}$ and $\tilde{\delta}_{\text {opt }}^{(b)}$ null out the corresponding biases so that

$$
\mathbf{E}\left[\frac{1}{Q_{1, a}}-\tilde{\delta}_{\mathrm{opt}}^{(a)} \frac{Q_{2, a}}{Q_{1, a}^{2}}\right]=\mathbf{E}\left[\frac{1}{Q_{1, b}}\right]
$$

In addition, from $(3.112), \tilde{\delta}_{\mathrm{opt}}^{(a)} \geq \tilde{\delta}_{\mathrm{opt}}^{(b)}$. Therefore, since $\hat{P}_{b}(\delta)=\frac{1}{Q_{1}(\delta)}$ is a monotonically increasing function, it follows that almost surely

$$
\frac{1}{Q_{1, a}} \geq \frac{1}{Q_{1, b}}
$$

Thus, for some positive constant $K$, it holds

$$
\frac{1}{Q_{1, a}}\left(\frac{1}{Q_{1, a}}-K\right) \geq \frac{1}{Q_{1, b}}\left(\frac{1}{Q_{1, b}}-K\right)
$$

Taking the expectation of both sides of (3.125) and rearranging the terms yields

$$
\mathbf{E}\left[\frac{1}{Q_{1, a}^{2}}\right]-K \mathbf{E}\left[\frac{1}{Q_{1, a}}-\frac{1}{Q_{1, b}}\right] \geq \mathbf{E}\left[\frac{1}{Q_{1, b}^{2}}\right]
$$

The second term on the left hand side of (3.126) is evaluated using (3.123). In addition, since $\frac{\tilde{\delta}_{\mathrm{opt}}^{(a)} Q_{2, a}}{Q_{1, a}^{2}}>0$, adding its expectation to the left hand side of $(3.126)$ 
does not change the inequality. Hence,

$$
\mathbf{E}\left[\frac{1}{Q_{1, a}^{2}}\right]-K \mathbf{E}\left[\frac{\tilde{\delta}_{\mathrm{opt}}^{(a)} Q_{2, a}}{Q_{1, a}^{2}}\right]+\mathbf{E}\left[\left(\frac{\tilde{\delta}_{\mathrm{opt}}^{(a)} Q_{2, a}}{Q_{1, a}^{2}}\right)^{2}\right] \geq \mathbf{E}\left[\frac{1}{Q_{1, b}^{2}}\right]
$$

Now we set $K=\frac{2\left(\hat{D}_{m}+\tilde{\delta}_{\mathrm{opt}}^{(a)}\right)}{m}>0$ and upper bound the left hand side of (3.127) by using (A.1) (from Appendix A) which implies that $\frac{1}{Q_{1, a}} \geq \frac{K}{2}$. Namely,

$$
\begin{aligned}
& \mathbf{E}\left[\frac{1}{Q_{1, a}^{2}}-2 \frac{\tilde{\delta}_{\mathrm{opt}}^{(a)} Q_{2, a}}{Q_{1, a}^{3}}+\left(\frac{\tilde{\delta}_{\mathrm{opt}}^{(a)} Q_{2, a}}{Q_{1, a}^{2}}\right)^{2}\right] \\
& \geq \mathbf{E}\left[\frac{1}{Q_{1, a}^{2}}-K \frac{\tilde{\delta}_{\mathrm{opt}}^{(a)} Q_{2, a}}{Q_{1, a}^{2}}+\left(\frac{\tilde{\delta}_{\mathrm{opt}}^{(a)} Q_{2, a}}{Q_{1, a}^{2}}\right)^{2}\right] \\
& \geq \mathbf{E}\left[\frac{1}{Q_{1, b}^{2}}\right]
\end{aligned}
$$

Recognizing that the left most hand side of (3.128) is the quadratic of $\hat{P}_{a}\left(\tilde{\delta}_{\text {opt }}^{(a)}\right)$ yields

$$
\mathbf{E}\left[\hat{P}_{a}^{2}\left(\tilde{\delta}_{\mathrm{opt}}^{(a)}\right)\right] \geq \mathbf{E}\left[\hat{P}_{b}^{2}\left(\tilde{\delta}_{\mathrm{opt}}^{(b)}\right)\right]
$$

Finally, from (3.123), which implies that $\mathbf{E}^{2}\left[\hat{P}_{a}\left(\tilde{\delta}_{\text {opt }}^{(a)}\right)\right]=\mathbf{E}^{2}\left[\hat{P}_{b}\left(\tilde{\delta}_{\mathrm{opt}}^{(b)}\right)\right]$, and (3.129), we obtain (3.122).

If the true power $P \leq \lim _{\delta \rightarrow \infty} \mathbf{E}\left[\hat{P}_{a}(\delta)\right]$, the squared biases corresponding to $\hat{P}_{a}$ and $\hat{P}_{b}$ at respectively $\tilde{\delta}_{\text {opt }}^{(a)}$ and $\tilde{\delta}_{\text {opt }}^{(b)}$ are equal. Given the inequality between their variances (3.122), we conclude that their MSE's are related as

$$
\operatorname{MSE}\left(\tilde{\delta}_{\text {opt }}^{(a)}\right) \geq \operatorname{MSE}\left(\tilde{\delta}_{\text {opt }}^{(b)}\right)
$$

We now invoke the conjecture that the MSE loss made by using $\tilde{\delta}_{\text {opt }}$ instead of $\delta_{\text {opt }}$ is negligible for both estimators. Therefore, the optimal MSE's are approximately related as

$$
\operatorname{MSE}\left(\delta_{\mathrm{opt}}^{(a)}\right) \geq \operatorname{MSE}\left(\delta_{\mathrm{opt}}^{(b)}\right)
$$




\subsubsection{Comparison based on Sensitivity to Optimal Diagonal Loading}

It has been observed in the simulation study that the power estimator $\hat{P}_{b}$ is more sensitive to optimal diagonal loading than the power estimator $\hat{P}_{a}$. Relying on the conjecture that the variance has negligible impact to the value of optimal diagonal loading, we define the sensitivity to optimal diagonal loading as the curvature of the squared bias around the diagonal loading which nulls out the squared bias.

Combining the results proved in Lemma 3.1 that

- the power estimator $\hat{P}_{b}$ is greater than the power estimator $\hat{P}_{a}$ for diagonal loading $\delta>0$;

- the power estimator $\hat{P}_{a}$ is monotonically non-decreasing and has zero slope as $\delta \rightarrow 0^{+}$and $\delta \rightarrow \infty$ and

- the power estimator $\hat{P}_{b}$ is strictly monotonically increasing for all $\delta \geq 0$,

and that the squared bias corresponding to $\hat{P}_{b}$ is minimized at smaller diagonal loading than that corresponding to $\hat{P}_{a}$, given by $(3.112)$, we conjecture that the power estimator $\hat{P}_{b}$ is more sensitive to optimal diagonal loading than the power estimator $\hat{P}_{a}$.

Proving this conjecture or finding the specific conditions under which it holds is a possible direction for future study.

\subsubsection{Numerical Validation}

This part verifies the theoretical result from previous part via simulations. The two scenarios are considered and the true power is evaluated in a standard way using (3.27).

The comparison between the MSE's of the two power estimators when the arrival process and number of sensors and snapshots is as specified by Scenario 1, is shown in Fig. 3-36. The MSE's in the top figure are evaluated at diagonal loadings which 
minimize the bias. The plots in the bottom figure compare the optimized MSE's. The simulation results in the top figure validate that the MSE corresponding to estimator $\hat{P}_{a}$ is lower bounded by the MSE corresponding to the estimator $\hat{P}_{b}$, both evaluated at the loadings which optimize corresponding squares biases. The bottom figure confirms the same conclusion for the optimized MSE's. Note that the MSE's of $\hat{P}_{a}$ and $\hat{P}_{b}$, compared in Fig. 3-36, are equal when steering close to the source directions. These are the ranges within which the optimal loading is close to zero and the two estimators are nearly identical.

The comparisons between the optimized MSE's and the MSE's evaluated at the diagonal loadings which optimize the corresponding squared biases of the two power estimators in Scenario 2, are shown in Fig. 3-37. In comparison to Scenario 1, a larger difference between the MSE's of the estimators $\hat{P}_{a}$ and $\hat{P}_{b}$ is observed when the number of snapshots is smaller than the number of sensors.

\subsection{Conclusions}

This chapter presents how two diagonally loaded, MPDR beamformer based spatial power spectrum estimators behave in the snapshot deficient regime.

The almost sure convergence of the considered power spectrum estimators to nonrandom limits when the number of snapshots and number of sensors grow large at the same rate is proved using the random matrix theory methods. The variance and estimation MSE of one of the power estimators are characterized under the assumption that the input process is Gaussian distributed.

Further, the dependences of the spatial power estimators, their expectations and variances on diagonal loading are studied. Unlike in standard theory, it is shown that due to specifics of the deficient sample support regime, the biases of both estimators are in general minimized for non-zero loadings. In addition, the MSE loss caused by using the minimizer of the squared bias as the optimal diagonal loading is negligible.

In addition, the dependence of optimal loading on steering direction is also analyzed. It is shown that when steering in the direction of a source which is well 

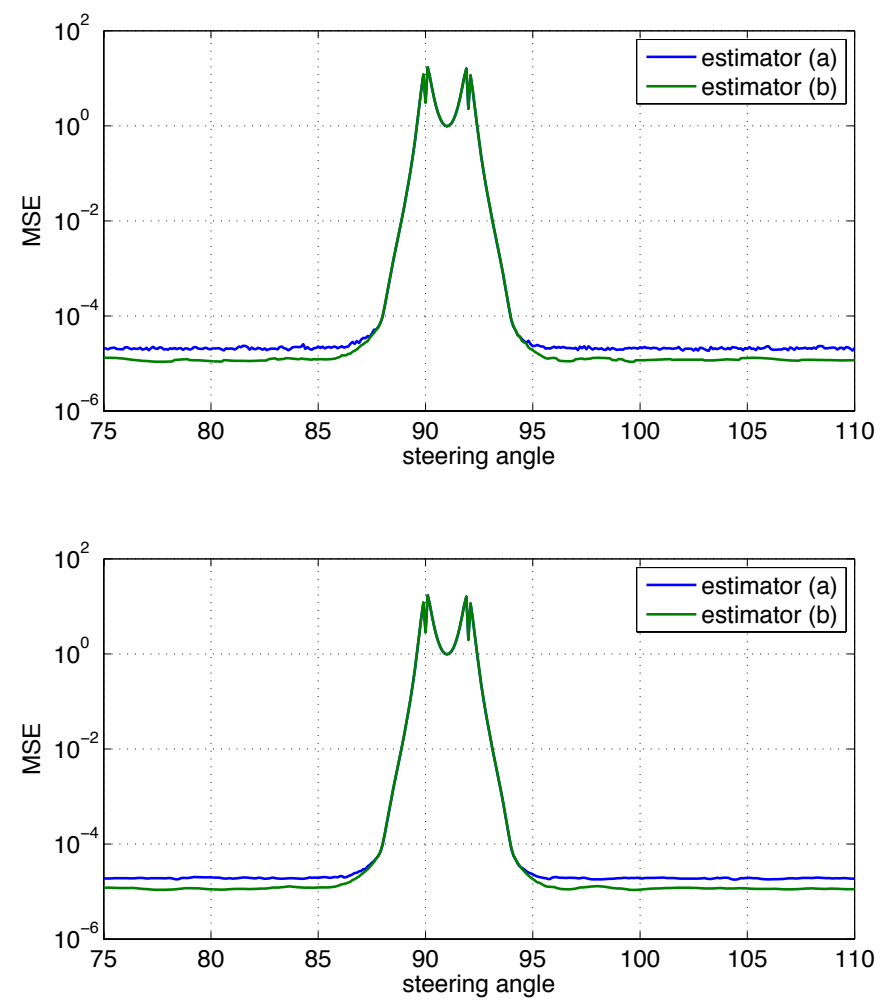

Figure 3-36: Scenario 1: Comparison between MSE performances of two power estimators. The MSE is evaluated at the loading which minimizes bias (top) and at the optimal diagonal loading (bottom). 

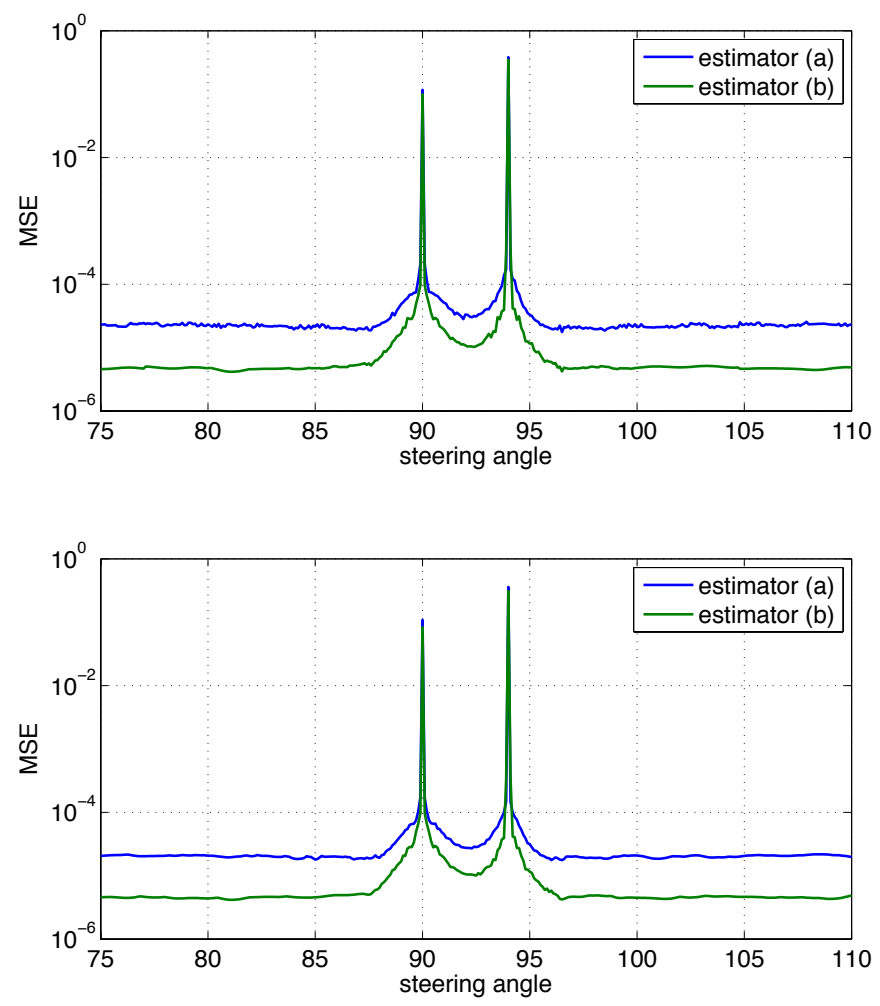

Figure 3-37: Scenario 2: Comparison between MSE performances of two power estimators. The MSE is evaluated at the diagonal loading which minimizes bias (top) and at the optimal diagonal loading (bottom). 
separated from the interferers, the optimal processor is a matched filter so $\delta_{\text {opt }} \rightarrow \infty$. If the sources are closely spaced, the optimal loading is finite. Furthermore, we show that when steering close to the source direction, the optimal loading is small and can even be zero, meaning that the optimal estimator tends to perform adaptation as much as possible, fully relying on the available data. On the other hand, when steering direction moves away from the source directions, the optimal loading tends to increase.

Finally, the performances of two power estimators are compared and it is proved that the optimized $\hat{P}_{\mathrm{b}}$ outperforms the optimized $\hat{P}_{\mathrm{a}}$; and the optimal $\delta$ is lower for estimator $\hat{P}_{\mathrm{b}}$ than it is for estimator $\hat{P}_{\mathrm{a}}$. However, the performance of estimator $\hat{P}_{\mathrm{b}}$ is more sensitive to the determination of the correct diagonal loading than is estimator $\hat{P}_{\mathrm{a}}$. All the presented results are validated via Monte-Carlo simulations.

As a possible future work, the stated conjecture about the negligible impact of variance on the value of optimal diagonal loading needs to be rigorously proved. Also, a rigorous sensitivity analysis of power estimators on optimal diagonal loading is needed to complete the comparison of power estimators. In addition, the results developed for Gaussian distributed snapshots could possibly be extended to more general snapshot statistics. Furthermore, we point out that the ultimate goal concerning the problem of diagonal loading for adaptive beamforming is to develop a scheme which determines the optimal diagonal loading based on the received data and steering direction. Also, the presented study on how spatial power spectrum estimation depends on diagonal loading could be applied to other estimation methods which rely on diagonal loading. Finally, the presented approach could possibly be applied for studying other regularization methods. 


\section{Chapter 4}

\section{Time-Varying Channel Tracking}

\subsection{Introduction}

The identification of an unknown channel from the input signal and output noisy observations using the Least Squares (LS) solution is a long-standing problem studied for different applications in the areas such as adaptive signal processing [27], estimation theory [29], machine learning [8] and communications [57]. The Recursive Least Squares (RLS) algorithm is an efficient implementation of the LS algorithm which has the ability to recursively update the estimate of the channel based on the most recent input-output sample pair. This leads to an application of the RLS algorithm for tracking slowly varying channels [27]. One of the areas where this application is particularly important is wireless communications where the receiver tracks the channel and detects the transmitted symbol based on the estimated channel impulse response [48], [39].

The tracking performance of the RLS algorithm has been extensively studied in the literature. As such, the steady-state mean-square estimation error is analyzed in [39], [19] and [32], where the impulse response of a channel is modeled as an autoregressive process. The tracking performance of the RLS algorithm in both transient and steady-state regimes is studied in [17].

The literature also reports performance studies of the RLS algorithm in some spe-

cific scenarios. As such, the steady state performance of the RLS based identification 
of an unknown LTI channel, modeled as a finite impulse response (FIR) filter whose length is shorter than the unknown channel length, is reported in [63]. The impact of round-off errors on tracking performance is studied in [1]. The performance of the RLS based tracking of a time-varying channel whose coefficients are modeled using Jakes' model is studied in [47]. The convergence properties of the RLS algorithm and their dependence on the initialization is analyzed in [36]. The performance of the RLS algorithm when used to detect symbols received from a frequency flat fading channel on an array is studied in [5].

The main challenge in the analysis of the performance of the RLS algorithm is to characterize the sample correlation matrix (SCM) of the input process and the inverse of this matrix when the number of observations is comparable to the dimension of the matrix (e.g., the size of the filter). The common approach in addressing this issue essentially includes approximating the SCM with the ensemble correlation matrix. While these matrices are approximately equal when the number of stationary observations is large, they might differ significantly in the observation deficient regime. The performance study presented in this chapter addresses this problem by exploiting the tools and results from random matrix theory (RMT).

A performance study of the RLS algorithm when it is used to track a channel which varies according to a first order Markov process in presented in this chapter. The expressions for signal prediction and channel estimation mean square errors (MSE) are derived and validated via simulations. The general results are applied for specific scenarios and as special cases we consider the behavior in the steady-state, performance of LS-based identification of linear time-invariant channel and performance of the sliding window RLS algorithm. Finally, several practical results such as those characterizing the optimal exponential forgetting factor in the exponentially weighted RLS or optimal averaging window length in the sliding window RLS algorithm are obtained.

The characterization of the Stieltjes transform of the channel estimation correlation matrix when the RLS and extended RLS algorithms are used to track a channel modeled as a random walk is reported in [56] and [55]. The transient behavior of 
the signal to interference plus noise ratio (SINR) at the output of the RLS estimator in a time-invariant channel is studied in [38]. The analysis therein is fairly general and the performance metric is given as a solution to a set of non-linear equations. In comparison to those results, we consider the signal prediction and channel estimation MSE's as performance metrics, study the transient behavior of the RLS algorithm directly and obtain the steady state results as a special case. In addition, we model the channel variations as a first order Markov process. Finally, by employing assumptions justifiable in the scenarios of practical interest, we obtain cleaner and, where possible, closed form characterizations.

This chapter is organized as follows. The background on channel tracking is summarized in Section 4.2. A general theory for the performance characterization of the LS based tracking of the first order Markov channel is developed in Section 4.3. The subsequent sections use the general results and specialize them for specific problems. As such, Section 4.4 studies the algorithm's performance in the steady state. An LTI system identification is treated in Section 4.5. The sliding window LS algorithm is studied in Section 4.6. The comparison between the exponentially weighted LS and the sliding window LS algorithm when used to track a first order Markov channel is discussed in Section 4.7. Section 4.8 concludes this chapter.

\subsection{Background}

The problem of tracking a time-varying channel using the Least Squares algorithm and the challenges associated with analyzing it are introduced in this section. In addition, relevant performance analysis results from the literature are given. Finally, the assumptions used in the theoretical analyzes and their justifications are presented.

\subsubsection{Problem Formulation}

Given the inputs to a finite impulse response (FIR) channel and the channel outputs corrupted by noise, the channel impulse response is estimated using the Least Squares (LS) algorithm. A channel of length $m$ has impulse response at time $n$ expressed as 
a vector $\mathbf{w}(n)$. The channel variation is modeled as a first order Markov process

$$
\mathbf{w}(n)=a \mathbf{w}(n-1)+\boldsymbol{\omega}(n),
$$

where $\boldsymbol{\omega}(n)$ is a zero mean i.i.d. process noise with correlation matrix $\sigma_{o}^{2} \mathbf{I}$. The channel impulse response at initial time $\mathbf{w}(0)$ is random and has identity covariance. A parameter $a<1$ is a state transition constant. For $a=1,(4.1)$ becomes a random walk model.

The channel input at time $n$ is the vector $\mathbf{u}(n)$ whose entries are input samples $u(k), k=n, n-1, \ldots, n-m+1$. The channel output is corrupted with an additive noise process $v(n)$ such that the channel output at time $n$ is

$$
d(n)=\mathbf{w}^{H}(n) \mathbf{u}(n)+v(n) .
$$

We assume the input and additive noise are independent. The noise process, $v(n)$, is i.i.d. with zero mean and variance $\sigma_{v}^{2}$. In addition, the input signal vectors $\mathbf{u}(n)$ are assumed to be zero-mean with correlation matrix $\mathbf{E}\left[\mathbf{u}(n) \mathbf{u}^{H}(n)\right]=\mathbf{R}$.

Given the input signal vectors $\mathbf{u}(i)$ and the corresponding desired outputs $d(i)$ up to time $n$, the estimated channel impulse response $\hat{\mathbf{w}}(n)$ is $[27]$

$$
\hat{\mathbf{w}}(n)=\hat{\mathbf{R}}^{-1}(n) \hat{\mathbf{r}}(n),
$$

where $\hat{\mathbf{R}}(n)$ is a sample correlation matrix (SCM), computed as

$$
\hat{\mathbf{R}}(n)=\sum_{i=1}^{n} \lambda^{n-i} \mathbf{u}(i) \mathbf{u}^{H}(i)+\lambda^{n} \delta \mathbf{I}
$$

and $\hat{\mathbf{r}}(n)$ is an input-output cross-correlation vector, given by

$$
\hat{\mathbf{r}}(n)=\sum_{i=1}^{n} \lambda^{n-i} \mathbf{u}(i) d^{*}(i) .
$$

A forgetting factor $\lambda \in(0,1)$, usually very close to 1 , accommodates the time- 
variability of the channel by suppressing the past observations not relevant for current estimation. The real-valued, non-negative quantity $\delta$ in (4.4) is a diagonal loading parameter which handles the start-up transient of the algorithm [27].

The performance of the tracking algorithm is measured via channel estimation error $\varepsilon(n)$,

$$
\boldsymbol{\varepsilon}(n)=\mathbf{w}(n)-\hat{\mathbf{w}}(n),
$$

and the signal prediction error $\xi(n)$,

$$
\xi(n)=d(n)-\hat{\mathbf{w}}^{H}(n-1) \mathbf{u}(n) .
$$

\subsubsection{Relevant Results}

The relevant results of the studies reported in the literature are derived and outlined in [27]. The performance analysis in [27] relies on the direct averaging assumption

and the approximation of the sample correlation matrix $\hat{\mathbf{R}}(n)$ of the stationary input process (such that the unity forgetting factor is used) with $\frac{1}{n} \hat{\mathbf{R}}(n) \approx \mathbf{R}$, which holds true when the number of observations $n$ is much larger than the channel length $m$.

When a channel vector varies according to an "almost" random walk model (a model from (4.1) with $a \rightarrow 1$ ) and the forgetting factor $\lambda$ is very close to 1 , the channel estimation error in a steady state is given by [27]

$$
\mathbf{E}\left[\|\varepsilon(n)\|_{2}^{2}\right] \rightarrow \frac{1-\lambda}{2} \sigma_{v}^{2} \operatorname{tr}\left\{\mathbf{R}^{-1}\right\}+\frac{1}{2(1-\lambda)} \operatorname{tr}\left\{\mathbf{R}_{\mathrm{o}}\right\}
$$

where \|\|$_{2}$ is the L2 norm of a vector, $\operatorname{tr}\{\}$ denotes the trace of a matrix and $\mathbf{R}_{\mathrm{o}}$ is a covariance matrix of the process noise $\boldsymbol{\omega}(n)$. By equating the error terms corresponding to the process and observation noise, the optimal value of the forgetting factor $\lambda_{\text {opt }}$ is evaluated as [27]

$$
\lambda_{\mathrm{opt}}=1-\frac{1}{\sigma_{v}}\left(\frac{\operatorname{tr}\left\{\mathbf{R}_{\mathrm{o}}\right\}}{\operatorname{tr}\left\{\mathbf{R}^{-1}\right\}}\right)^{\frac{1}{2}} .
$$

For the case when the system is LTI, i.e., $a=1$ and $\mathbf{R}_{\mathrm{o}}=\mathbf{0}$, and $\lambda=1$, the 
expressions for the mean-square values of the channel estimation and signal prediction errors are for $n \gg m$ given by [27]

$$
\mathbf{E}\left[\|\varepsilon(n)\|_{2}^{2}\right] \approx \frac{1}{n} \sigma_{v}^{2} \operatorname{tr}\left\{\mathbf{R}^{-\mathbf{1}}\right\}
$$

and

$$
\mathbf{E}\left[\|\xi(n)\|_{2}^{2}\right] \approx \frac{1}{n} \sigma_{v}^{2} \operatorname{tr}\left\{\mathbf{R}^{-1}\right\}+\sigma_{v}^{2} .
$$

The performance analysis given in [27] assumes the number of observations $n$ is much larger than the channel length $m$. However, when $n$ and $m$ are of the same order, the estimated and true correlation matrices may differ significantly. The theoretical characterization of the LS algorithm, developed in this chapter with the use of random matrix theory methods, does not require $n$ be large. Furthermore, compared to [27], the performance of the LS-based tracking of a broader class of channel variations is characterized.

\subsubsection{Assumptions and Remarks}

The main assumptions used in the performance characterization presented in this chapter are as follows.

(1) The forgetting factor, $\lambda$, is assumed to be close to 1 in the derivation of both the channel estimation and signal prediction MSE.

(2) The state transition parameter, $a$, is assumed to be close to 1 in the derivation of signal prediction MSE.

(3) The input observation vectors $\mathbf{u}(n)$ are assumed to be independent and identically distributed.

Note that the analysis in [27] uses the same assumptions.

Although these assumptions might seem somewhat too restrictive, the simulations

show that the derived expressions are valid for the ranges of $\lambda$ and $a$ that are in fact of practical importance. This is shown in Figures 4-4 and 4-17 and emphasized in the 
corresponding discussions. In short, the RLS algorithm fails to accurately track a first order Markov varying channel whose state transition parameter $a$ is not sufficiently close to 1. Also, the effective averaging window size corresponding to a value of forgetting factor $\lambda$ not sufficiently close to 1 might be too short with respect to the number of unknown coefficients, which in turn does not lead to acceptable tracking performance.

The third assumption is used in the evaluation of the moments of the SCM using random matrix methods, in particular Theorem 2.1, as elaborated in Section 2.4. In reality, the observation vectors at the input of an FIR filter are not independent. However, the results obtained via simulations conducted such that the consecutive observation vectors are shifted with respect to each other, confirm the validity of the derived expressions. In addition, this assumption is fairly common in adaptive filter theory.

As a final remark, random matrix theory provides tools for evaluating the performance metrics of our interest without restricting $\lambda$ and $a$ be sufficiently closed to 1. However, our intention is to characterize the performance using a relatively clean and, where possible, closed form expressions. Such expressions are derived under the stated assumptions which are justified in the regime of practical importance where the RLS algorithm is able to track the channel reasonably well.

\subsection{Performance Analysis}

This channel estimation and signal prediction MSE's associated with the LS-based tracking of first order Markov channel with deficient sample support are characterized in this section. 


\subsubsection{Channel Estimation Error}

A channel impulse response, modeled as a first order Markov process (4.1), is at time $n$ expressed in terms of the initial impulse response $\mathbf{w}(0)$ as

$$
\mathbf{w}(n)=a^{n} \mathbf{w}(0)+\sum_{i=1}^{n} a^{n-i} \boldsymbol{\omega}(i) .
$$

Substituting (4.2) into (4.5), the cross-correlation vector is expressed as

$$
\hat{\mathbf{r}}(n)=\sum_{i=1}^{n} \lambda^{n-i} \mathbf{u}(i)\left(\mathbf{u}^{H}(i) \mathbf{w}(i)+v^{*}(i)\right) .
$$

After substituting (4.13) into (4.3) and using (4.12), the estimated channel vector becomes

$$
\begin{aligned}
\hat{\mathbf{w}}(n)= & \hat{\mathbf{R}}^{-1}(n) \sum_{i=1}^{n} a^{i} \lambda^{n-i} \mathbf{u}(i) \mathbf{u}^{H}(i) \mathbf{w}(0) \\
& +\hat{\mathbf{R}}^{-1}(n) \sum_{i=1}^{n} \lambda^{n-i} \mathbf{u}(i) \mathbf{u}^{H}(i) \sum_{j=1}^{i} a^{i-j} \boldsymbol{\omega}(j) \\
& +\hat{\mathbf{R}}^{-1}(n) \sum_{i=1}^{n} \lambda^{n-i} \mathbf{u}(i) v^{*}(i) .
\end{aligned}
$$

The channel estimation error, after substitution of (4.12) and (4.14) into (4.6), can be decomposed into three terms

$$
\varepsilon(n)=\varepsilon_{1}(n)+\varepsilon_{2}(n)+\varepsilon_{3}(n),
$$

where $\varepsilon_{1}(n)$ is the error induced by the initial channel response

$$
\varepsilon_{1}(n)=\left[a^{n} \mathbf{I}-\hat{\mathbf{R}}^{-1}(n) \sum_{i=1}^{n} a^{i} \lambda^{n-i} \mathbf{u}(i) \mathbf{u}^{H}(i)\right] \mathbf{w}(0),
$$


$\varepsilon_{2}(n)$ is the error induced by the random portion of the channel dynamics (process noise),

$$
\varepsilon_{2}(n)=\sum_{i=1}^{n} a^{n-i} \omega(i)-\hat{\mathbf{R}}^{-1}(n) \sum_{i=1}^{n} \lambda^{n-i} \mathbf{u}(i) \mathbf{u}^{H}(i) \sum_{j=1}^{i} a^{i-j} \omega(j),
$$

and $\varepsilon_{3}(n)$ is the observation noise induced error

$$
\varepsilon_{3}(n)=-\hat{\mathbf{R}}^{-1}(n) \sum_{i=1}^{n} \lambda^{n-i} \mathbf{u}(i) v^{*}(i)
$$

Due to assumed independence of the initial channel response, input process and observation and process noises, the three error terms are uncorrelated. In the following, the expected norms of these error terms are evaluated. The identities $\operatorname{tr}\{\mathbf{A B}\}=\operatorname{tr}\{\mathbf{B A}\}$ and $\mathbf{E}[\operatorname{tr}\{\mathbf{A}\}]=\operatorname{tr}\{\mathbf{E}[\mathbf{A}]\}$ are used.

The mean square value of the initial channel response induced error $\varepsilon_{1}(n)$ is, after some algebraic manipulation, given by

$$
\begin{aligned}
\mathbf{E}\left[\left\|\varepsilon_{1}(n)\right\|_{2}^{2}\right]= & m a^{2 n}-2 a^{n} \sum_{i=1}^{n} \lambda^{n-i} a^{i} \mathbf{E}\left[\mathbf{u}^{H}(i) \hat{\mathbf{R}}^{-1}(n) \mathbf{u}(i)\right] \\
& +\sum_{i=1}^{n} \sum_{j=1}^{n} a^{i+j} \lambda^{2 n-i-j} \mathbf{E}\left[\operatorname{tr}\left\{\mathbf{u}(i) \mathbf{u}^{H}(i) \hat{\mathbf{R}}^{-2}(n) \mathbf{u}(j) \mathbf{u}^{H}(j)\right\}\right]
\end{aligned}
$$

Recall that the initial channel response vector $\mathbf{w}(0)$ is assumed to be random and of identity correlation. Therefore, its correlation matrix gives rise to multiplication with $m$ in the first term and is absorbed in the trace operator in the last term of (4.19).

The channel dynamics induced error $\varepsilon_{2}(n)$ can be expressed as a sum of the uncorrelated error terms (which are denoted $\mathbf{e}_{k}(n)$ )

$$
\varepsilon_{2}(n)=\sum_{k=1}^{n} \mathbf{e}_{k}(n)
$$


where

$$
\mathbf{e}_{k}(n)=\left(a^{n-k} \mathbf{I}-\hat{\mathbf{R}}^{-1}(n) \sum_{i=k}^{n} \lambda^{n-i} a^{i-k} \mathbf{u}(i) \mathbf{u}^{H}(i)\right) \boldsymbol{\omega}(k)
$$

The mean square value of $\varepsilon_{2}(n)$ is thus

$$
\left.\mathbf{E}\left\|\varepsilon_{2}(n)\right\|_{2}^{2}\right]=\sum_{k=1}^{n} \mathbf{E}\left[\left\|\mathbf{e}_{k}(n)\right\|_{2}^{2}\right]
$$

where the expression for $\mathbf{E}\left[\left\|\mathbf{e}_{k}(n)\right\|_{2}^{2}\right]$, obtained after some algebraic manipulation, is given by

$$
\begin{aligned}
\mathbf{E}\left[\left\|\mathbf{e}_{k}(n)\right\|_{2}^{2}\right]= & \sigma_{o}^{2} m a^{2(n-k)}-2 \sigma_{o}^{2} a^{n-k} \sum_{i=k}^{n} a^{i-k} \lambda^{n-i} \mathbf{E}\left[\mathbf{u}^{H}(i) \hat{\mathbf{R}}^{-1}(n) \mathbf{u}(i)\right] \\
& +\sigma_{o}^{2} \sum_{i=k}^{n} \sum_{j=k}^{n} a^{i+j-2 k} \lambda^{2 n-i-j} \mathbf{E}\left[\operatorname{tr}\left\{\mathbf{u}(i) \mathbf{u}^{H}(i) \hat{\mathbf{R}}^{-2}(n) \mathbf{u}(j) \mathbf{u}^{H}(j)\right\}\right]
\end{aligned}
$$

Finally, the expected norm of the observation noise induced error $\varepsilon_{3}(n)$ is given by

$$
\mathbf{E}\left[\left\|\varepsilon_{3}(n)\right\|_{2}^{2}\right]=\sigma_{v}^{2} \sum_{i=1}^{n} \lambda^{2(n-i)} \mathbf{E}\left[\mathbf{u}^{H}(i) \hat{\mathbf{R}}^{-2}(n) \mathbf{u}(i)\right] .
$$

Overall, the channel estimation MSE is

$$
\left.\left.\mathbf{E}\|\varepsilon(n)\|_{2}^{2}\right]=\sum_{i=1}^{3} \mathbf{E}\left\|\varepsilon_{i}(n)\right\|_{2}^{2}\right]
$$

\subsubsection{Signal Prediction Error}

Substituting (4.2) into (4.7) and using model (4.1), the signal prediction error is expressed as

$$
\xi(n)=\left(a \mathbf{w}^{H}(n-1)-\hat{\mathbf{w}}^{H}(n-1)\right) \mathbf{u}(n)+\omega^{H}(n) \mathbf{u}(n)+v(n) .
$$


The simulation results, presented in Section 4.6.2, imply that a channel varying according to a first order Markov model is not tractable unless $a$ is close to 1 . Consequently, to obtain a compact representation of $\xi(n)$, it is assumed that $a \approx 1$ so that

$$
\xi(n) \approx \varepsilon^{H}(n-1) \mathbf{u}(n)+\boldsymbol{\omega}^{H}(n) \mathbf{u}(n)+v(n) .
$$

While $\xi(n)$ does not explicitly depend on $a$, this dependence is implicitly accounted for via the dependence of $\varepsilon(n)$ on the state transition parameter, $a$.

If the input process has identity correlation, the signal prediction MSE is, using (4.27), given by

$$
\mathbf{E}\left[\|\xi(n)\|^{2}\right] \approx \mathbf{E}\left[\|\varepsilon(n-1)\|_{2}^{2}\right]+m \sigma_{o}^{2}+\sigma_{v}^{2}
$$

Thus, to evaluate the signal prediction MSE, we need to characterize the first term in (4.28).

\subsubsection{Theoretical Prediction of Unknown Quantities}

The MSE values of the error terms expressed with (4.19), (4.23) and (4.24) depend on two quantities which we define as

$$
\begin{aligned}
V_{k}(n, m, i) & =\mathbf{E}\left[\mathbf{u}^{H}(i) \hat{\mathbf{R}}^{-k}(n) \mathbf{u}(i)\right] \\
W(n, m, i, j) & =\mathbf{E}\left[\operatorname{tr}\left\{\mathbf{u}(i) \mathbf{u}^{H}(i) \hat{\mathbf{R}}^{-2}(n) \mathbf{u}(j) \mathbf{u}^{H}(j)\right\}\right]
\end{aligned}
$$

The index $k$ in $V_{k}$ can be either 1 or 2 . These quantities are studied in the following parts and approximately expressed in terms of the expected moments of the SCM $\tilde{M}_{k}(m, n)$ defined as the expected normalized trace of the powers of the SCM inverse, that is

$$
\tilde{M}_{k}(m, n)=\frac{1}{m} \mathbf{E}\left[\operatorname{tr}\left\{\hat{\mathbf{R}}^{-k}(n)\right\}\right] .
$$


We are interested in the expected moments whose corresponding index $k$ is 1 or 2 . Note that $\tilde{M}_{0}(m, n)=1$.

Two cases are considered. In the first case, the ensemble correlation matrix of the input process $\mathbf{R}$ is unconstrained and the expected moment $\tilde{M}_{1}(m, n)$ is approximated for $n>m$ and $\delta=0$ with the limiting moment, as is elaborated in Section 2.7. The limiting moment $\bar{M}_{1}$ corresponding to the exponentially weighted SCM with forgetting factor $\lambda$ and $\delta=0$ is characterized in Section 2.5 and given by (2.37). Hence, the expected first moment is approximately given by the solution to the fixed point equation

$$
\frac{1}{\tilde{M}_{1}(m, n)}=\sum_{k=1}^{n} \frac{\lambda^{n-k}}{\frac{1}{m} \operatorname{tr}\left\{\mathbf{R}^{-1}\right\}+m \lambda^{n-k} \tilde{M}_{1}(m, n)}
$$

Note that in a special case when $\lambda=1, \tilde{M}_{1}(m, n)$ is given in a closed form

$$
\tilde{M}_{1}(m, n)=\frac{1}{m(n-m)} \operatorname{tr}\left\{\mathbf{R}^{-1}\right\}
$$

In the second case, $\mathbf{R}=\mathbf{I}$ and there is no restriction on the number of observation vectors, $n$. This means that $n$ can be smaller than $m$ and a positive diagonal loading factor $\delta$, is needed. The expected moments with the corresponding index $k=1,2$ are approximated with the limiting moments, evaluated in closed form in Section 2.5 and given by

$$
\tilde{M}_{1}(m, n)=\max \left(0,1-\frac{1}{c}\right) \frac{1}{\delta}+\frac{\sqrt{\delta^{2}+(m-n)^{2}+2 \delta(m+n)}-|n-m|-\delta}{2 \delta m}
$$

and

$$
\tilde{M}_{2}(m, n)=\max \left(0,1-\frac{1}{c}\right) \frac{1}{\delta^{2}}-\frac{|n-m|}{2 \delta^{2} m}+\frac{(m-n)^{2}+\delta(m+n)}{2 \delta^{2} m \sqrt{\delta^{2}+(m-n)^{2}+2 \delta(m+n)}} .
$$




\section{Evaluation of $V_{k}$}

We approximate in this part the quadratic form $V_{k}$ for $k=1,2$. As a remark, an exact asymptotic characterization can be obtained by using the tools from random matrix theory, such as the integration by parts formula and Poincare-Nash inequality. However, our goal is to obtain a simple, yet accurate characterizations by employing the assumption justified in practical scenarios.

A rectangular window $(\lambda=1)$ is considered first. In that case, each input snapshot $\mathbf{u}(i), i=1,2, \ldots, n$ contributes to the sample correlation matrix $\hat{\mathbf{R}}(n)$ equally, with unit weight. Due to symmetry, $V_{k}(n, m, i)$ does not depend on the index $i$, i.e., $V_{k}(n, m, i)=V_{k}(n, m)$. Therefore,

$$
\begin{aligned}
V_{k}(n, m) & =\frac{1}{n} \sum_{i=1}^{n} V_{k}(n, m) \\
& =\frac{1}{n} \sum_{i=1}^{n} \mathbf{E}\left[\mathbf{u}^{H}(i) \hat{\mathbf{R}}^{-k}(n) \mathbf{u}(i)\right] \\
& =\frac{1}{n} \mathbf{E}\left[\operatorname{tr}\left\{\hat{\mathbf{R}}^{-k}(n) \sum_{i=1}^{n} \mathbf{u}(i) \mathbf{u}^{H}(i)\right\}\right] \\
& =\frac{1}{n} \mathbf{E}\left[\operatorname{tr}\left\{\hat{\mathbf{R}}^{-k}(n)(\hat{\mathbf{R}}(n)-\delta \mathbf{I})\right\}\right] \\
& =\frac{m}{n}\left(\tilde{M}_{k-1}(m, n)-\delta \tilde{M}_{k}(m, n)\right) .
\end{aligned}
$$

When exponential weighting is employed, different observation vectors are weighted differently, so that $V_{k}(n, m, i)$ depends on the observation index $i$. In the absence of a better approach, we assume forgetting factor $\lambda$ is very close to 1 such that $V_{k}(n, m, i) \approx V_{k}(n, m)$. This approximation is further justified because $k$ can only take on values 1 or 2 (larger values of $k$ make the approximation less accurate). Therefore,

$$
\sum_{i=1}^{n} \lambda^{n-i} V_{k}(n, m, i) \approx \frac{1-\lambda^{n}}{1-\lambda} V_{k}(n, m) .
$$


Without the approximation, the expression becomes

$$
\begin{aligned}
\sum_{i=1}^{n} \lambda^{n-i} V_{k}(n, m, i) & =\mathbf{E}\left[\operatorname{tr}\left\{\hat{\mathbf{R}}^{-k}(n) \sum_{i=1}^{n} \lambda^{n-i} \mathbf{u}^{H}(i) \mathbf{u}(i)\right\}\right] \\
& =m\left(\tilde{M}_{k-1}(m, n)-\delta \lambda^{n} \tilde{M}_{k}(m, n)\right) .
\end{aligned}
$$

Therefore, equating the right-hand sides of (4.37) and (4.38),

$$
V_{k}(n, m, i) \approx m \frac{1-\lambda}{1-\lambda^{n}}\left(\tilde{M}_{k-1}(m, n)-\delta \lambda^{n} \tilde{M}_{k}(m, n)\right) .
$$

Letting $\lambda \rightarrow 1$ and noting that $(1-\lambda) /\left(1-\lambda^{n}\right) \rightarrow 1 / n$, (4.36) is recovered from (4.39).

In addition, note that the expected moments $\tilde{M}_{k}$ from (4.39) correspond to the exponentially weighted SCM.

To confirm the reasoning for $\lambda=1$ and approximation for $\lambda$ close to $1, V_{k}(n, m, i)$ for $i=1,2, \ldots, n$ and $k=1,2$ are evaluated using Monte-Carlo simulations. The corresponding plots are shown in Figures 4-1 and 4-2 for respectively $\lambda=1$ and $\lambda=0.995$. As can be observed, the conclusion that $V_{k}(n, m, i)$ does not depend on $i$ when $\lambda=1$ is validated in Fig. 4-1.

On the other hand, the approximation $V_{2}(n, m, i) \approx V_{2}(n, m)$ is less accurate when $\lambda<1$, as shown in Fig. 4-2. In the absence of more accurate and algebraically simple characterization for $V_{k}(n, m, i)$ when $\lambda<1$, we use (4.39) in the analysis. The validation of the results obtained in the later parts confirm that approximation (4.39) is acceptable. As a final note, the approximation is getting more accurate as $\lambda$ is approaching one.

\section{Evaluation of $W$}

The approximation of quantity $W$ is derived in this part. As for $V_{k}$, our goal is to characterize this quantity with relatively simple and accurate expressions by utilizing the assumption justified in realistic scenarios.

Based on whether the indices $i$ and $j$ in $W_{k}(n, m, i, j)$ are equal or not, two new 

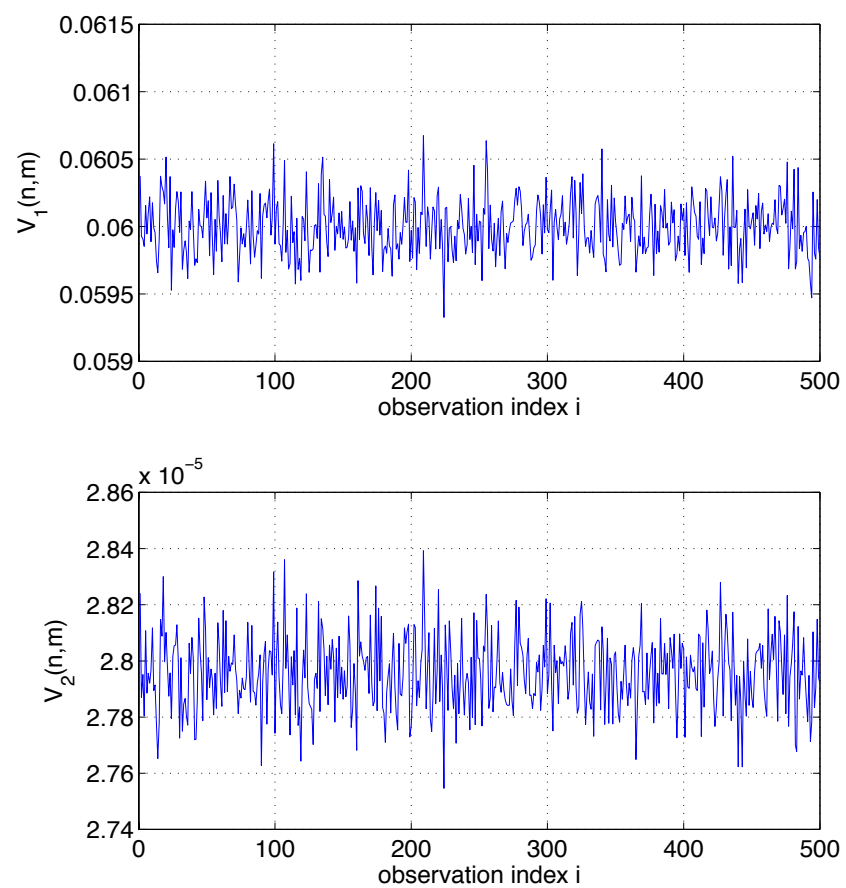

Figure 4-1: $V_{1}$ (top figure) and $V_{2}$ (bottom figure) versus observation index $i$ for $n=500, m=30$ and $\lambda=1$.

quantities are defined

$$
X(n, m, i)=\mathbf{E}\left[\operatorname{tr}\left\{\mathbf{u}(i) \mathbf{u}^{H}(i) \hat{\mathbf{R}}^{-2}(n) \mathbf{u}(i) \mathbf{u}^{H}(i)\right\}\right]
$$

and

$$
Y(n, m, i, j)=\mathbf{E}\left[\operatorname{tr}\left\{\mathbf{u}(i) \mathbf{u}^{H}(i) \hat{\mathbf{R}}^{-2}(n) \mathbf{u}(j) \mathbf{u}^{H}(j)\right\}\right], i \neq j .
$$

When using the rectangular window, $X(n, m, i)=X(n, m)$ and $Y(n, m, i, j)=$ $Y\left(n, m, i_{0}, j_{0}\right)$ for the same reason as was stated for the case of $V_{k}(n, m, i)$. Here, $i_{0}$ and $j_{0}$ are fixed and not equal.

The quantity $X$ is expressed as

$$
X(n, m)=\mathbf{E}\left[\|\mathbf{u}(i)\|_{2}^{2} \mathbf{u}^{H}(i) \hat{\mathbf{R}}^{-2}(n) \mathbf{u}(i)\right]
$$

and we assume that $\|\mathbf{u}(i)\|_{2}^{2}$ and $\mathbf{u}^{H}(i) \hat{\mathbf{R}}^{-2}(n) \mathbf{u}(i)$ are independent random variables 

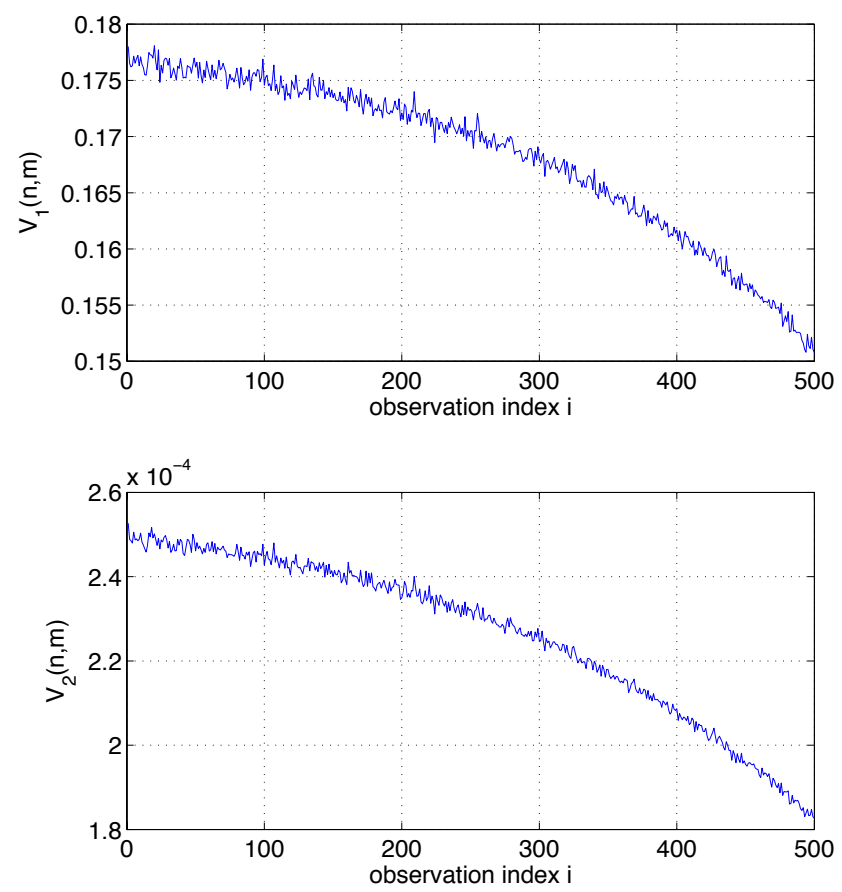

Figure 4-2: $V_{1}$ (top figure) and $V_{2}$ (bottom figure) versus observation index $i$ for $n=500, m=30$ and $\lambda=0.995$.

when $n$ and $m$ are of the same order. Namely, $\mathbf{u}^{H}(i) \hat{\mathbf{R}}^{-2}(n) \mathbf{u}(i)$ is a norm of a vector obtained by rotating $\mathbf{u}(i)$ with the eigenvectors of $\hat{\mathbf{R}}(n)$ and scaling the entries of the resulting vector with the squared inverse of the eigenvalues of $\hat{\mathbf{R}}(n)$. While rotating a vector with an orthogonal matrix has no impact on its norm, scaling its entries by randomly chosen eigenvalues from broad support (because $n$ and $m$ are comparable) makes $\mathbf{u}^{H}(i) \hat{\mathbf{R}}^{-2}(n) \mathbf{u}(i)$ and $\|\mathbf{u}(i)\|_{2}^{2}$ approximately independent. Hence, $X$ is approximated as

$$
X(n, m) \approx \operatorname{tr}\{\mathbf{R}\} V_{2}(n, m)
$$

The theoretical approximation (4.43) is compared with the simulation results for different $n$ and $m=30$ and the plots are shown in Fig. 4-3. Excellent agreement is obtained even for large values of $n$. The input process in this test is Gaussian distributed. A similar agreement is also obtained for a uniformly distributed binary input sequence.

When an exponential weighting is employed, $\lambda$ is assumed to be very close to 


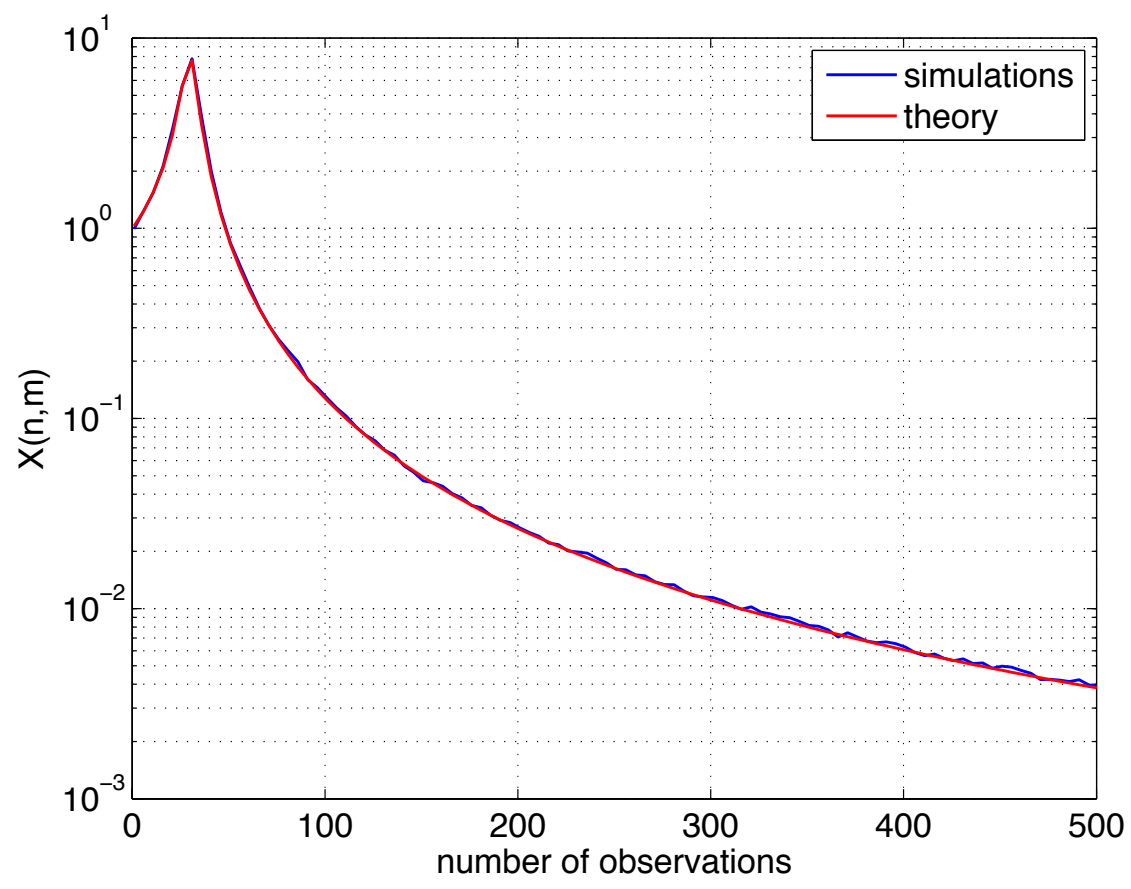

Figure 4-3: Comparison between the Simulated and Approximated $X(n, 30)$

1. Thus, $X(n, m, i) \approx X(n, m), Y(n, m, i, j) \approx Y\left(n, m, i_{o}, j_{o}\right)$, where $i_{o} \neq j_{o}$, and $X(n, m)$ is evaluated using (4.43).

To evaluate $Y$, two possible decompositions of the weighed sums involving $W(n, m, i, j)$ are given by

$$
\begin{aligned}
\sum_{i=1}^{n} \sum_{j=1}^{n} \lambda^{2 n-i-j} W(n, m, i, j) & \approx \sum_{i=1}^{n} \lambda^{2(n-i)} X(n, m)+\sum_{i=1}^{n} \sum_{i \neq j=1}^{n} \lambda^{2 n-i-j} Y\left(n, m, i_{o}, j_{o}\right) \\
& =\frac{1-\lambda^{2 n}}{1-\lambda^{2}} X(n, m)+2 \frac{\left(1-\lambda^{n}\right)\left(\lambda-\lambda^{n}\right)}{(1-\lambda)^{2}(1+\lambda)} Y\left(n, m, i_{o}, j_{o}\right)
\end{aligned}
$$

and

$$
\begin{aligned}
\sum_{i=1}^{n} \sum_{j=1}^{n} \lambda^{2 n-i-j} W(n, m, i, j) & =\mathbf{E}\left[\operatorname{tr}\left\{\sum_{i=1}^{n} \lambda^{n-i} \mathbf{u}(i) \mathbf{u}^{H}(i) \hat{\mathbf{R}}^{-2}(n) \sum_{j=1}^{n} \lambda^{n-j} \mathbf{u}(j) \mathbf{u}^{H}(j)\right\}\right] \\
& =m\left(\tilde{M}_{0}(m, n)-2 \delta \lambda^{n} \tilde{M}_{1}(n, m)+\delta^{2} \lambda^{n} \tilde{M}_{2}(n, m)\right)
\end{aligned}
$$


By equating the right hand sides of (4.44) and (4.45), we obtain the relation between $X$ and $Y$

$$
\frac{1-\lambda^{2 n}}{1-\lambda^{2}} X+2 \frac{\left(1-\lambda^{n}\right)\left(\lambda-\lambda^{n}\right)}{(1-\lambda)^{2}(1+\lambda)} Y=m\left(\tilde{M}_{0}-2 \delta \lambda^{n} \tilde{M}_{1}+\delta^{2} \lambda^{n} \tilde{M}_{2}\right)
$$

and evaluate $Y$ for $X$ approximated using (4.43).

Substituting $\lambda=1$ (rectangular window) into (4.46), the exact relation between $X$ and $Y$ reads as

$$
n X+n(n-1) Y=m\left(\tilde{M}_{o}-2 \delta \tilde{M}_{1}+\delta^{2} \tilde{M}_{2}\right)
$$

\subsection{Channel Tracking in Steady State}

The theoretical framework developed in the previous section is used in this section to analyze the performance of the exponentially weighted LS algorithm in the steady state, i.e., when the number of input observation vectors $n \rightarrow \infty$. In the first part we evaluate the channel estimation MSE. The numerical validation of the derived expression is given in the second part. In addition, the assumption that the forgetting factor $\lambda$ is close to 1 , used in the derivations, is justified.

\subsubsection{Performance Analysis}

The steady state channel estimation MSE of the exponentially weighted LS algorithm is evaluated in this part. Note that the channel varies according to the first order Markov model and the non-unitary forgetting factor $\lambda$ limits the estimator memory and enables the algorithm to accommodate time-variability.

Taking the limit $n \rightarrow \infty$ of (4.19), we conclude that the contribution of the error term due to the initial channel vector to the overall channel estimation MSE disappears in the steady state. This result is intuitively appealing.

Substituting the approximate expressions for $V_{k}$ and $W$ into (4.23), the obtained expression into (4.22) and taking the limit $n \rightarrow \infty$ of the result, the power of the 
error induced by the random portion of the channel dynamics is in the steady state given by

$$
P_{\mathrm{dyn}}^{\infty}=\frac{m \sigma_{o}^{2}}{(1+a)(1-a \lambda)}\left(2 \lambda-1+\frac{\operatorname{tr}\{\mathbf{R}\}}{1+\lambda} \tilde{M}_{1}^{\infty}\right),
$$

Similarly, substituting the approximations for $V_{k}$ and $W$ into (4.24) and taking the limit $n \rightarrow \infty$, the power of the error induced by the observation noise is given by

$$
P_{\text {noise }}^{\infty}=\frac{m \sigma_{v}^{2}}{1+\lambda} \tilde{M}_{1}^{\infty}
$$

The powers (4.48) and (4.49) are given in terms of the expected first moment if the SCM in the steady state, $\tilde{M}_{1}^{\infty}$, defined as

$$
\tilde{M}_{1}^{\infty}=\lim _{n \rightarrow \infty} \mathbf{E}\left[\tilde{M}_{1}(m, n)\right]
$$

Note that here only the number of observation vectors $n$ becomes large, while $m$ is kept constant. However, since $\lambda<1$, the effective number of observation vectors is finite so that the SCM might not approach ensemble correlation matrix $\mathbf{R}$.

Taking the limit $n \rightarrow \infty$ of (4.32), the moment $M_{1}^{\infty}$ is characterized with the fixed point equation

$$
\frac{1}{\tilde{M}_{1}^{\infty}}=\sum_{k=1}^{\infty} \frac{\lambda^{k}}{\frac{1}{m} \operatorname{tr}\left\{\mathbf{R}^{-1}\right\}+m \lambda^{k} \tilde{M}_{1}^{\infty}} .
$$

\subsubsection{Numerical Validation and Discussion}

The derived expression for the channel estimation MSE in the steady state is tested by comparing its agreement with the estimation error obtained via Monte-Carlo simulations and characterization (4.8) from [27]. A simulated zero-mean Gaussian input process with non-identity correlation is processed through a first order Markov varying channel with the state transition parameter $a=0.99$ and process noise variance 0.01. The output signal is corrupted with white noise such that the signal-to-noise ratio (SNR) of the output signal is $10 \mathrm{~dB}$. The channel is estimated using the LS algorithm with different values for the forgetting factor $\lambda$.

The top figure in Fig. 4-4 shows the comparison between the theory, simulations 
and characterization (4.8) derived in [27] on a relatively wide range of forgetting factors $\lambda$. The bottom figure in Fig. 4-4 shows the comparison between the theory and simulations on a narrower range of forgetting factors $\lambda$.

Recall that the theoretical expressions (4.49) and (4.48) are derived under the assumption that $\lambda$ is close to 1 . Also, characterization (4.8) is derived under the same assumption, with the additional assumption that the state transition parameter $a \rightarrow 1$. As can be observed from the figures, the derived characterization accurately predicts the performance when $\lambda$ is close to 1 . Next we argue that the range of forgetting factors $\lambda$ not sufficiently close to 1 has no practical importance.

Namely, given that in this example the algorithm is estimating $m=30$ unknown channel coefficients, a value of forgetting factor $\lambda$ whose corresponding effective averaging window size is not long enough to accommodate the adaptation of $m=30$ coefficients, is not to be used in a practical scenario. Assuming that in the worst case scenario we need at least one observation per unknown coefficient, the forgetting factor $\lambda$ should be such that the corresponding effective averaging window size $n_{\text {eff }}$ is 30 . A widely used rule of thumb relates the value of forgetting factor $\lambda$ and effective averaging window size $n_{\mathrm{eff}}$ as $n_{\mathrm{eff}}=1 /(1-\lambda)$. This implies that the effective averaging window length of 30 corresponds to $\lambda=0.9667$. Therefore, if $\lambda<0.9667$, the algorithm fails to reasonably well track the channel variations and these forgetting factors are not to be used in a practical application. Finally, the comparison in Fig. 4-4 confirms that the theoretical characterization derived under the assumption that $\lambda$ is close to 1 , is valid for the range of forgetting factors that are of practical importance.

As a final remark, note that the value of the smallest $\lambda$ to be used in a practical scenario is a conservative estimate because more than one observation per dimension is needed for achieving a satisfactory performance. 

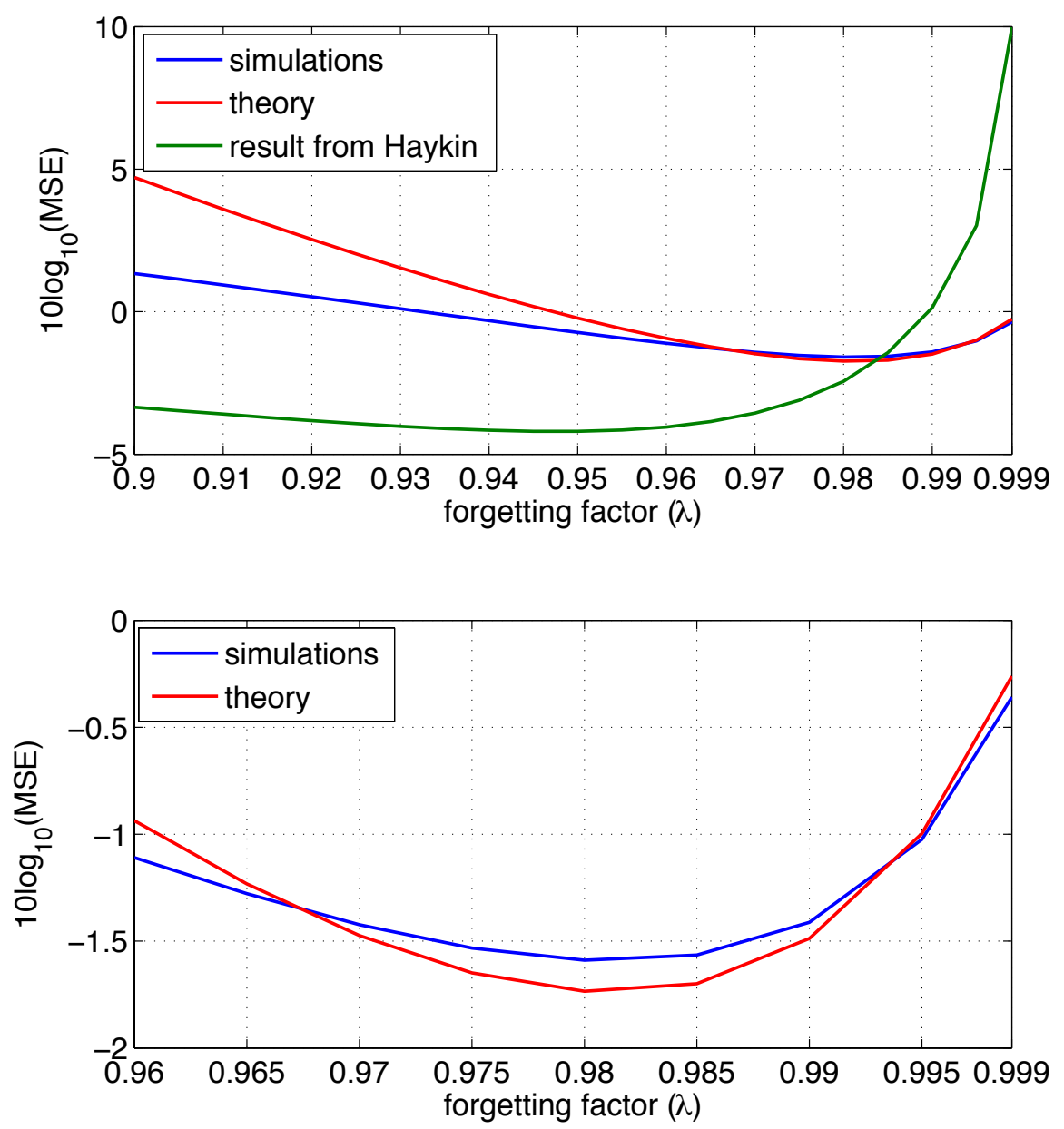

Figure 4-4: Steady state channel estimation MSE for first-order Markov channel. The input process has unconstrained covariance and the channel length is 30. Top figure compares our theoretical prediction, result from Haykin's text (4.8) and simulations on a wide range of forgetting factors. The bottom figure compares our theoretical prediction and simulations on a narrower range of forgetting factors. The values on the vertical axis in both figures are relative to the L2 norm of the channel vector in steady state, which is 15 in this case. 


\subsection{Linear-Time Invariant Channel Identification}

This section considers a linear time-invariant (LTI) channel identification problem with the LS algorithm. The channel estimation and signal prediction mean square errors are evaluated and the analytical expressions are validated via Monte-Carlo simulations. We show that at low SNR, a deterioration in the performance appears when the number of observations is close to the channel length. This effect is characterized and explained.

\subsubsection{Performance Analysis}

\section{Channel Estimation Error}

A performance characterization of the LS-based identification of a linear, time invariant channel is obtained from the theory developed in Section 4.3. An LTI channel is described with $a=1$ and $\sigma_{o}^{2}=0$ in model (4.1), i.e., the channel vector $\mathbf{w}(n)=\mathbf{w}(0)=\mathbf{w}_{0}$. Due to channel invariability in time, the input observations are rectangularly windowed, i.e., $\lambda=1$. Substituting $a=1, \sigma_{o}^{2}=0$ and $\lambda=1$ into (4.19), (4.22) and (4.24), the power of the error induced by the initial channel vector is given by

$$
\mathbf{E}\left[\left\|\varepsilon_{1}(n)\right\|_{2}^{2}\right]=m \delta^{2} \tilde{M}_{2}(m, n),
$$

where $m$ is the channel length, $\delta$ is the diagonal loading and $\tilde{M}_{2}(m, n)$ is the expected first moment corresponding to the SCM. The power of the error induced by the observation noise is given by

$$
\mathbf{E}\left[\left\|\varepsilon_{3}(n)\right\|_{2}^{2}\right]=m \sigma_{v}^{2}\left(\tilde{M}_{1}(m, n)-\delta \tilde{M}_{2}(m, n)\right),
$$

where $\sigma_{v}^{2}$ is the variance of the observation noise and $\tilde{M}_{2}(m, n)$ is the expected second moment corresponding to the SCM. Note that the error due to channel dynamics $\varepsilon_{2}(n)$ is 0 . Overall, the channel estimation MSE is

$$
\mathbf{E}\left[\|\varepsilon(n)\|_{2}^{2}\right]=m \sigma_{v}^{2} \tilde{M}_{1}(n, m)-m \delta\left(\sigma_{v}^{2}-\delta\right) \tilde{M}_{2}(n, m) .
$$


When the input process has identity covariance $\mathbf{I}$, the functional dependence of the channel estimation MSE on the number of observations $n$, channel length $m$ and diagonal loading parameter $\delta$ is obtained by substituting the closed form expressions (4.34) and (4.35) for $\tilde{M}_{1}(m, n)$ and $\tilde{M}_{2}(m, n)$ into (4.54).

The expectation of the second moment $\tilde{M}_{2}(m, n)$ appears in the expression for the channel estimation MSE in a product with the diagonal loading $\delta$, which has negligible impact on the performance when $n>m$. Consequently, for $n>m$, the impact of the terms depending on $\delta$ is neglected. Substituting (4.33) into (4.54) yields

$$
\mathbf{E}\left[\|\varepsilon(n)\|_{2}^{2}\right]=\frac{\sigma_{v}^{2}}{n-m} \sum_{k=1}^{m} \frac{1}{\lambda_{k}}
$$

where $\lambda_{k}$ 's are the eigenvalues of the ensemble correlation matrix.

\section{Signal Prediction Error}

When the input process has identity correlation I, (4.28) gives the signal prediction MSE in terms of the channel estimation MSE.

For an input process of unconstrained correlation $\mathbf{R}$, the signal prediction MSE is derived by assuming the diagonal loading $\delta$ is zero (meaning that the number of observations is greater than the channel length). Using (4.27) we get

$$
\mathbf{E}\left[\xi(n) \xi^{*}(n)\right]=\operatorname{tr}\left\{\mathbf{E}\left[\varepsilon(n-1) \varepsilon^{H}(n-1) \mathbf{R}\right]\right\}+\sigma_{v}^{2}
$$

The correlation matrix of the channel estimation error could, for the LTI channel identification problem and $\delta=0$, be shown to be

$$
\begin{aligned}
\mathbf{E}\left[\boldsymbol{\varepsilon}(n) \boldsymbol{\varepsilon}^{H}(n)\right] & =\sigma_{v}^{2} \sum_{i=1}^{n} \mathbf{E}\left[\hat{\mathbf{R}}^{-1}(n) \mathbf{u}(i) \mathbf{u}^{H}(i) \hat{\mathbf{R}}^{-1}(n)\right] \\
& =\sigma_{v}^{2} \mathbf{E}\left[\hat{\mathbf{R}}^{-1}(n)\right]
\end{aligned}
$$

If the input process is Gaussian, the SCM is Wishart distributed. Using the expression for the expectation of the inverse of the Wishart matrix [27], the covariance 
matrix of the channel estimation error becomes

$$
\mathbf{E}\left[\varepsilon(n) \varepsilon^{H}(n)\right]=\frac{\sigma_{v}^{2}}{n-m-1} \mathbf{R}^{-1}
$$

For a general non-Gaussian case, the expectation of the SCM inverse is evaluated using the RMT results. Namely, it is shown in Section 2.5.2 that if the order of the SCM $m$ and the number of observations $n$ grow large at the same rate such that $\frac{m}{n} \rightarrow c \in(0,1)$, the inverse of the rectangularly windowed SCM almost surely converges to the scaled inverse of the ensemble correlation matrix (2.54). Noting that the SCM $\hat{\mathbf{R}}$ defined in (4.4) with $\lambda=1$ and $\delta=0$ is a scaled version of the model for rectangularly windowed SCM considered in Section 2.5.2, the convergence result (2.54) is rewritten as

$$
\left(\frac{1}{n} \hat{\mathbf{R}}(n)\right)^{-1} \rightarrow \frac{1}{1-c} \mathbf{R}^{-1} \quad \text { a.s. }
$$

As elaborated in Section 2.7, the expected inverse of the SCM is approximated with the limiting quantity in (4.59) such that

$$
\mathbf{E}\left[\hat{\mathbf{R}}^{-1}(n)\right] \approx \frac{\mathbf{R}^{-1}}{n-m}
$$

Finally, from (4.58) and (4.56) the signal prediction MSE for Gaussian input process is

$$
\mathbf{E}\left[|\xi(n)|^{2}\right]=\frac{n-2}{n-m-2} \sigma_{v}^{2}
$$

which is valid for $n>m+2$. Note that this constraint carries no practical insight.

Similarly, substituting (5.26) into (4.57) and the obtained result into (4.56) yields an approximation for the signal prediction MSE when the received signal has general statistics,

$$
\mathbf{E}\left[|\xi(n)|^{2}\right] \approx \frac{n-1}{n-m-1} \sigma_{v}^{2}
$$

which is valid for $n>m+1$. Note that (5.28) also approximates the Gaussian case. 


\section{A-Posteriori Signal Prediction MSE}

Although not treated in the development of the general theory, the channel time invariability renders relatively simple analysis of the a-posteriori signal prediction error. This error, denoted $\xi_{a}(n)$, is defined as

$$
\begin{aligned}
\xi_{a}(n) & =d(n)-\hat{\mathbf{w}}^{H}(n) \mathbf{u}(n) \\
& =(\mathbf{w}(0)-\hat{\mathbf{w}}(n))^{H} \mathbf{u}(n)+v(n) .
\end{aligned}
$$

Substituting $a=1$ and $\lambda=1$ into (4.14) and plugging the obtained result into (4.63) yields

$$
\xi_{a}(n)=\delta \hat{\mathbf{w}}_{0}^{H} \hat{\mathbf{R}}^{-1}(n) \mathbf{u}(n)+v(n)-\sum_{i=1}^{n} \mathbf{u}^{H}(i) \hat{\mathbf{R}}^{-1}(n) \mathbf{u}(n) v(i) .
$$

Denoting the first and last terms in (4.64) with $e_{1}(n)$ and $e_{2}(n)$ and noting that $e_{1}(n)$ and $e_{2}(n)$ as well as $e_{1}(n)$ and $v(n)$ are independent, the mean-square value of the a-posteriori prediction error becomes

$$
\mathbf{E}\left[\left\|\xi_{a}(n)\right\|^{2}\right]=\mathbf{E}\left[\left\|e_{1}(n)\right\|^{2}\right]+\mathbf{E}\left[\left\|e_{2}(n)\right\|^{2}\right]+\sigma_{v}^{2}-2 \mathbf{E}\left[e_{2}(n) v^{*}(n)\right]
$$

The power of $e_{1}(n)$ is evaluated as

$$
\begin{aligned}
\mathbf{E}\left[\left\|e_{1}(n)\right\|^{2}\right] & =\mathbf{E}\left[\delta^{2} \mathbf{w}_{\mathbf{0}}{ }^{H} \hat{\mathbf{R}}^{-1}(n) \mathbf{u}(n) \mathbf{u}^{H}(n) \hat{\mathbf{R}}^{-1}(n) \mathbf{w}_{\mathbf{0}}\right] \\
& =m \delta^{2} \tilde{M}_{2}(m, n) .
\end{aligned}
$$

Similarly, the expression for the power of $e_{2}(n)$ is obtained via

$$
\begin{aligned}
\mathbf{E}\left[\left\|e_{2}(n)\right\|^{2}\right] & =\sigma_{v}^{2} \sum_{i=1}^{n} \mathbf{E}\left[\mathbf{u}^{H}(n) \hat{\mathbf{R}}^{-1}(n) \mathbf{u}(i) \mathbf{u}^{H}(i) \hat{\mathbf{R}}^{-1}(n) \mathbf{u}(n)\right] \\
& =m \sigma_{v}^{2}\left[\tilde{M}_{1}(m, n)-\delta \tilde{M}_{2}(m, n)\right] .
\end{aligned}
$$


The cross-power between $e_{2}(n)$ and noise sample $v(n)$ is

$$
\begin{aligned}
\mathbf{E}\left[e_{2}(n) v^{*}(n)\right] & =\mathbf{E}\left[\sum_{i=1}^{n} \mathbf{u}^{H}(i) v(i) \hat{\mathbf{R}}^{-1}(n) \mathbf{u}(n) v^{*}(n)\right] \\
& =m \sigma_{v}^{2} \tilde{M}_{1}(m, n) .
\end{aligned}
$$

Finally, substituting (4.66), (4.67) and (4.68) into (4.65) yields

$$
\mathbf{E}\left[\left\|\xi_{a}(n)\right\|^{2}\right]=\sigma_{v}^{2}-m \sigma_{v}^{2} \tilde{M}_{1}(m, n)+m\left(\delta-\sigma_{v}^{2}\right) \delta \tilde{M}_{2}(m, n) .
$$

If the input process is of identity correlation, the moments $\tilde{M}_{1}$ and $\tilde{M}_{2}$ are calculated through (4.34) and (4.35). If, on the other hand, the input observations are of arbitrary correlation $\mathbf{R}$, the a-posteriori MSE is computed for $n>m$ by neglecting $\delta$ and evaluating moment $\tilde{M}_{1}$ using (4.33).

\subsubsection{Theoretical versus Numerical Results}

To test the accuracy of the derived expressions, the mean-square values of the channel estimation and signal prediction errors are computed via Monte-Carlo simulations and used as the ground truth. A channel with impulse response $\mathbf{w}_{0}$ processes the input signal and the output is corrupted with the observation noise whose variance is $\sigma_{v}^{2}$.

The plots of the mean-square values of the channel estimation and signal prediction errors versus number of observations when the signal-to-noise ratio is $40 \mathrm{~dB}$ are shown respectively in Fig. 4-5 and Fig. 4-6. The input data stream is an i.i.d. standard Gaussian random process, a diagonal loading parameter used in the RLS algorithm is $\delta=0.01$ and the channel has length 30 .

The corresponding plots for $\mathrm{SNR}=5 \mathrm{~dB}, \delta=0.1$ and i.i.d. standard Gaussian input are given in Fig. 4-7 and Fig. 4-8. When the input process is a uniform bipolar input sequence with $\mathrm{SNR}=5 \mathrm{~dB}$ and $\delta=0.1$, the comparison between the simulations and theoretical predictions of the channel estimation and signal prediction MSE's are shown in Fig. 4-9 and Fig. 4-10.

When the input is a correlated multivariate Gaussian process, the corresponding 


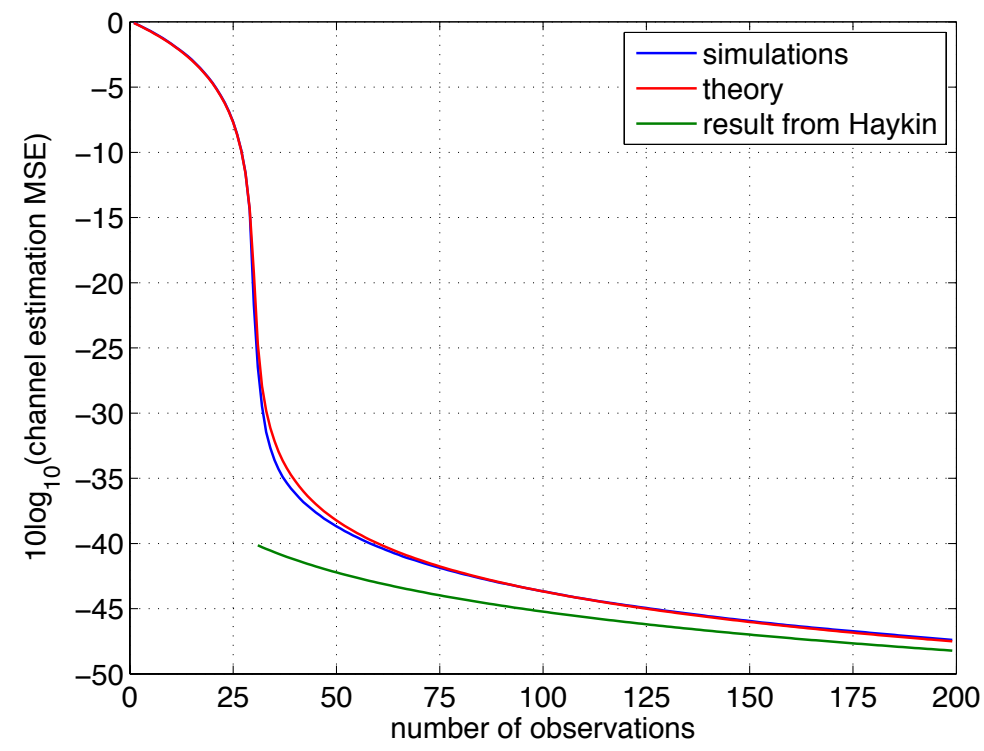

Figure 4-5: Channel estimation MSE vs number of observations. The input process is uncorrelated Gaussian, the channel length is 30 and $\mathrm{SNR}=40 \mathrm{~dB}$. The corresponding result from Haykin's text is (4.10).

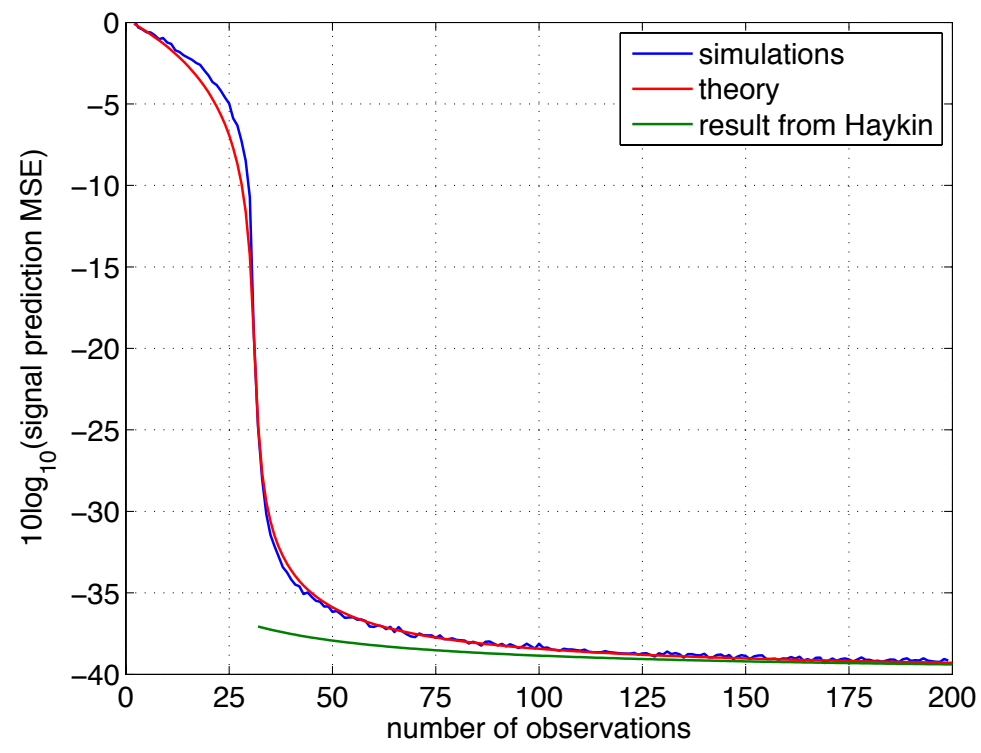

Figure 4-6: Signal prediction MSE vs number of observations for independent Gaussian input, channel of 30 taps and $40 \mathrm{~dB}$ SNR. The result from Haykin's text is (4.11). 


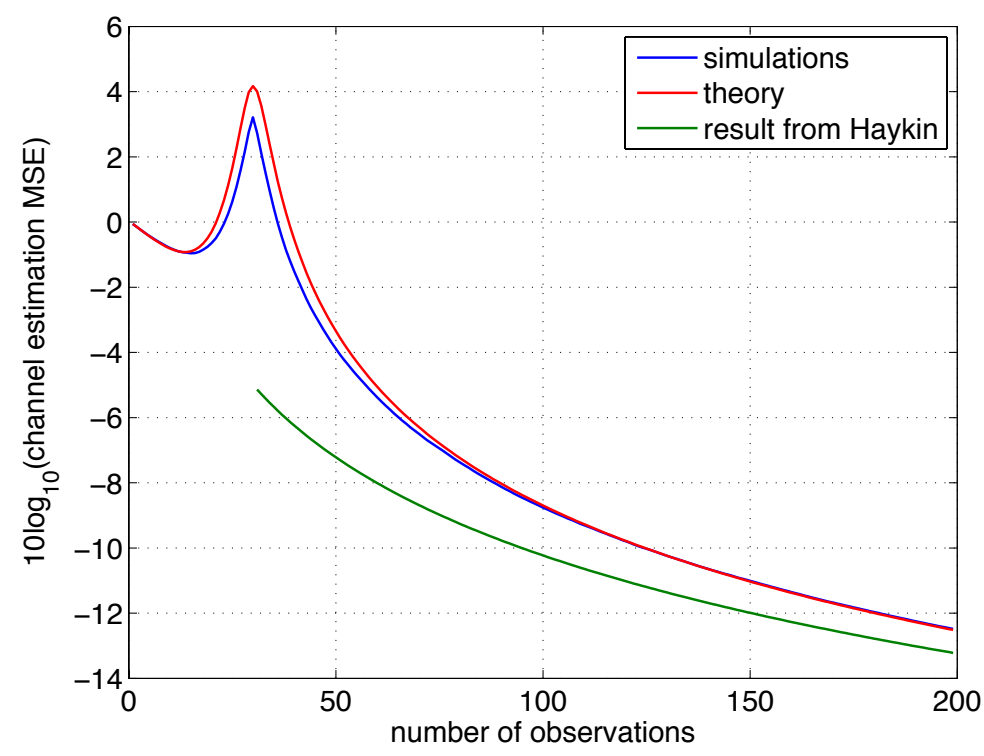

Figure 4-7: Channel estimation MSE vs number of observations for independent Gaussian input, channel of length 30 and SNR of $5 \mathrm{~dB}$.

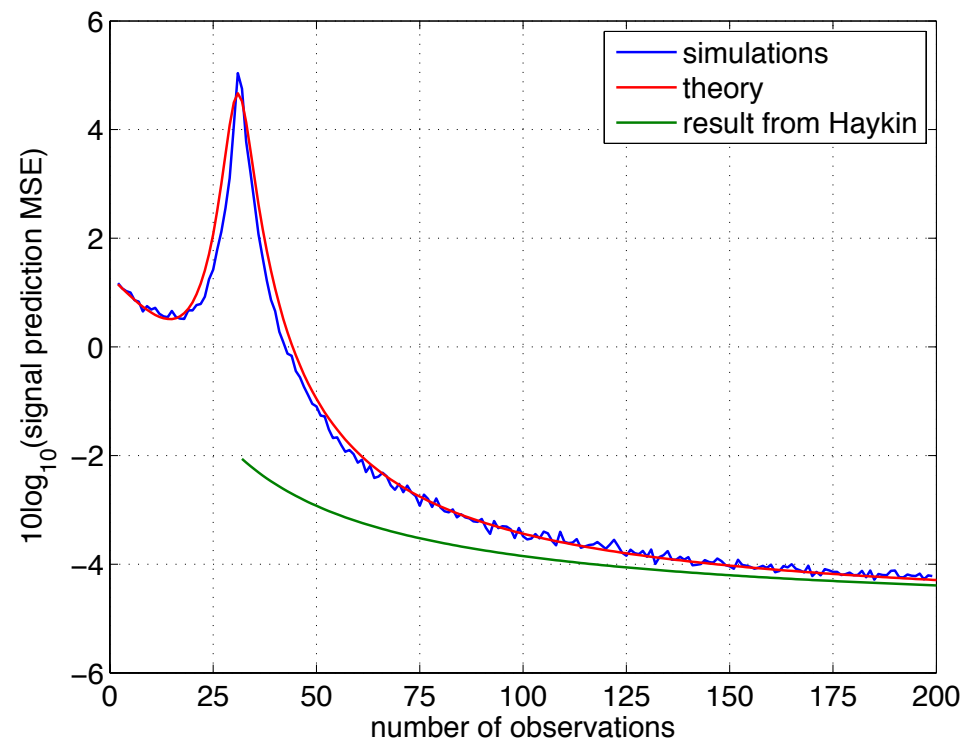

Figure 4-8: Signal prediction MSE vs number of observations for independent Gaussian input, channel of length 30 and SNR of $5 \mathrm{~dB}$. 


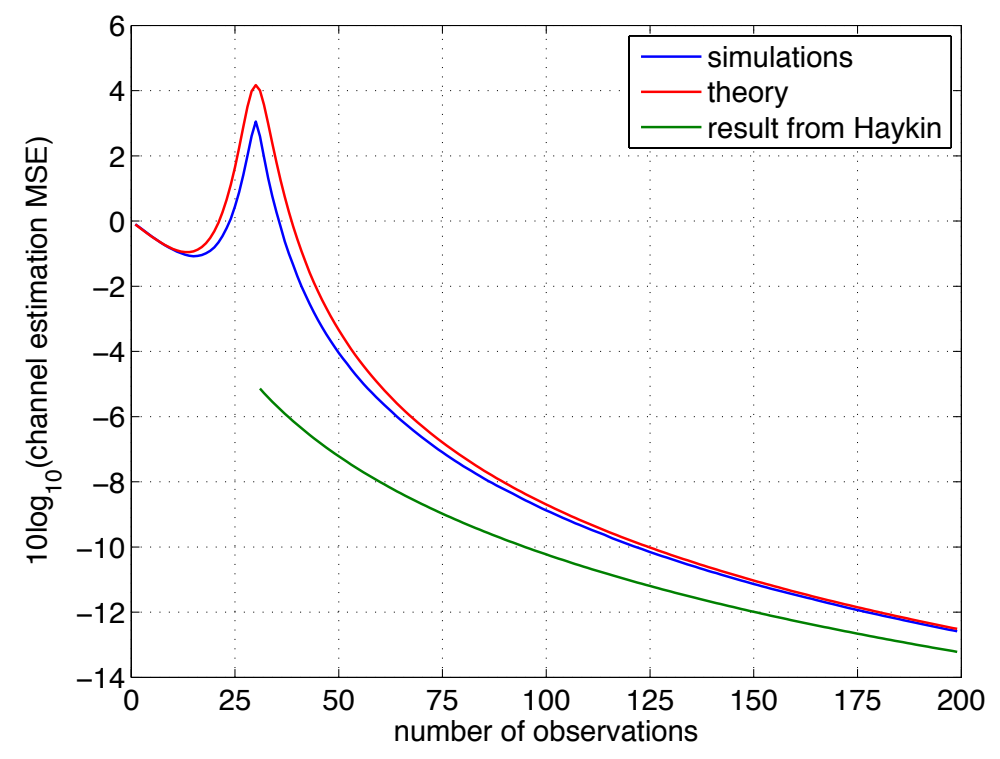

Figure 4-9: Channel estimation MSE vs number of observations for bipolar uniform input, channel of length 30 and SNR of $5 \mathrm{~dB}$.

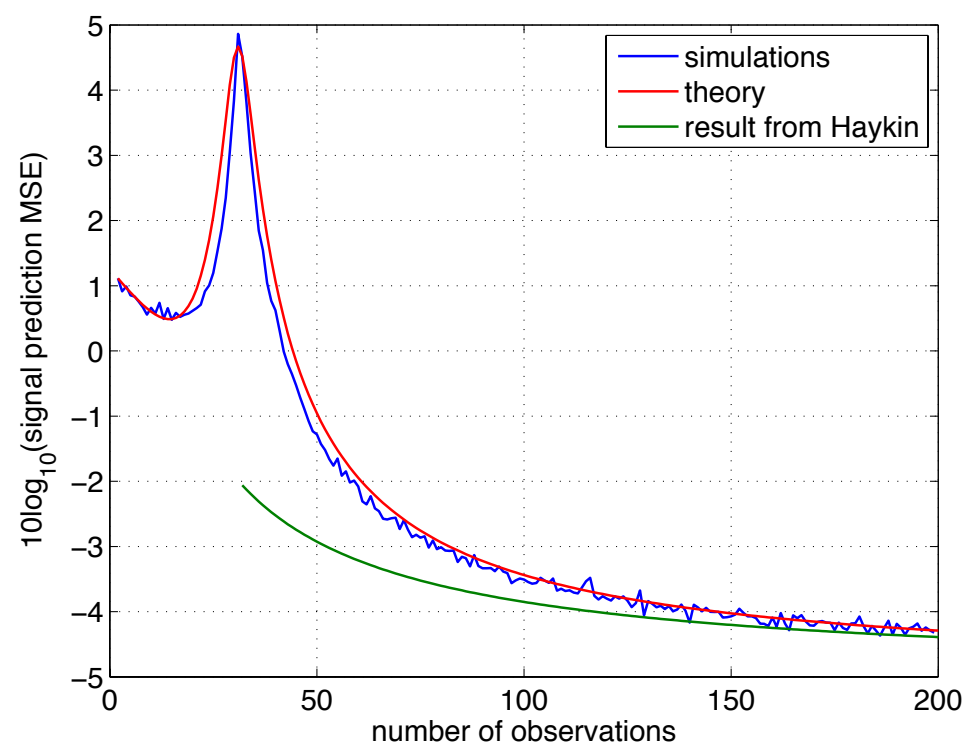

Figure 4-10: Signal prediction MSE vs number of observations for bipolar uniform input, channel of length 30 and SNR of $5 \mathrm{~dB}$. 


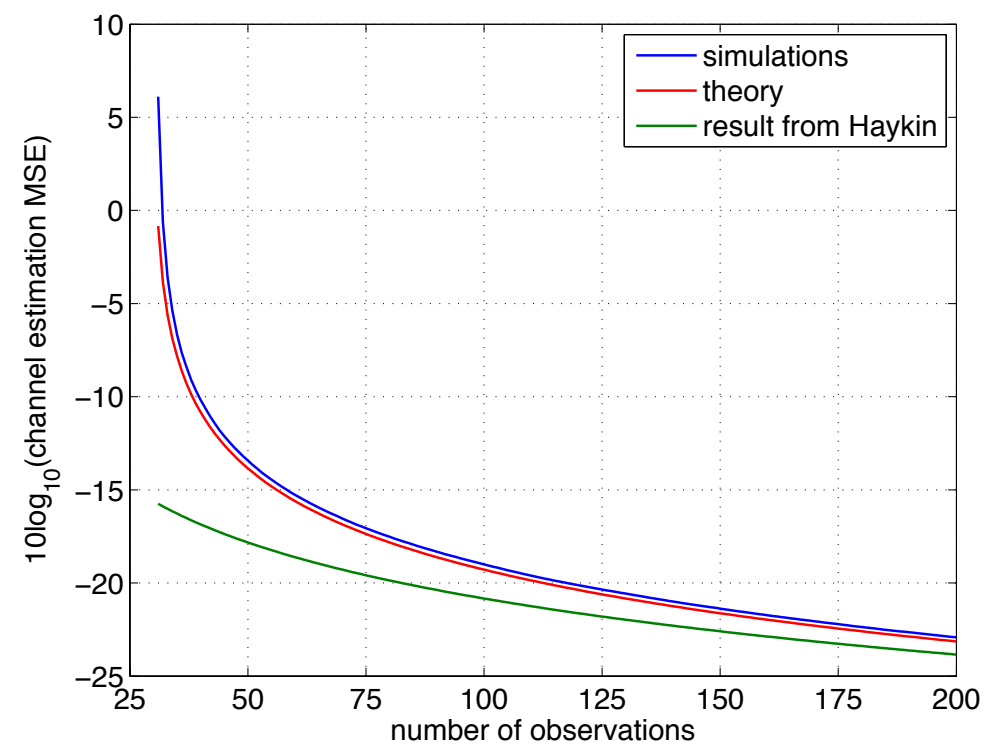

Figure 4-11: Channel estimation MSE versus number of observation when the input process has unconstrained correlation matrix, channel is of length 30 and SNR is 10 $\mathrm{dB}$.

performance curves for $\mathrm{SNR}=10 \mathrm{~dB}$ and channel length 30 are shown in Fig. 4-11.

The figures also include the plots of the corresponding analytical expressions derived in Haykin's text [27] and given in (4.10) and (4.11). The presented plots show that the derived expressions closely match the experimental curves. In addition, Figures 4-7, 4-8, 4-9 and 4-10 reveal deterioration in the algorithm's performance when the SNR is relatively small and the number of observations becomes close to the channel length. This happens irrespective of the statistics of the input signal. We explain this effect in the following part.

\subsubsection{Performance Deterioration}

In the following analysis the input process is assumed i.i.d., i.e., $\mathbf{R}=\mathbf{I}$. To explain why the channel estimation and signal prediction errors exhibit a bump when the number of observations $n$ is around the channel length $m$, the algorithm's update equation is exploited [27]

$$
\hat{\mathbf{w}}(n)=\hat{\mathbf{w}}(n-1)+\hat{\mathbf{R}}^{-1}(n) \mathbf{u}(n) \xi_{a}(n) .
$$




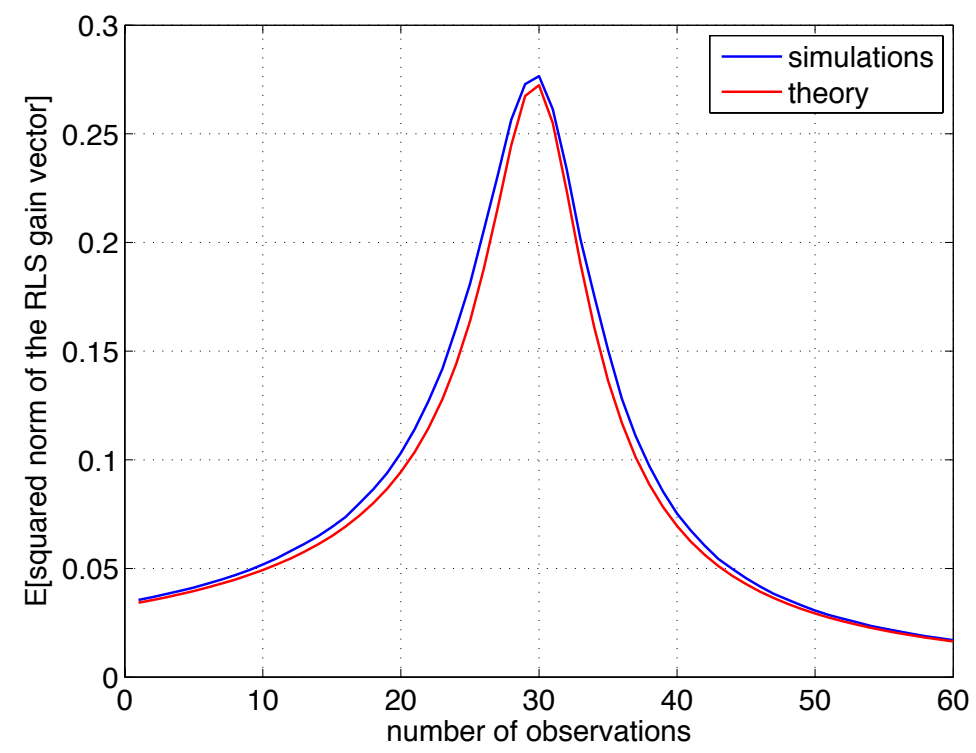

Figure 4-12: Expected squared norm of the gain vector vs number of observations $n$. The channel length is 30 .

A gain vector $\mathbf{k}(n)=\hat{\mathbf{R}}^{-1}(n) \mathbf{u}(n)$ gives the direction of the update of the estimated channel impulse response. Its expected squared norm is

$$
\mathbf{E}\left[\mathbf{k}^{H}(n) \mathbf{k}(n)\right]=\mathbf{E}\left[\mathbf{u}^{H}(n) \hat{\mathbf{R}}^{-2}(n) \mathbf{u}(n)\right]=V_{2}(n, m)
$$

The comparison between the Monte-Carlo simulated $\mathbf{E}\left[\|\mathbf{k}(n)\|_{2}^{2}\right]$ and its analytical characterization given in (4.71) and (4.36) is shown in Fig. 4-12. Aside from getting very close match between the simulations and theory, it is observed that the squared norm of the gain vector exhibits a bump when the number of observations $n$ is close to the channel length $m$.

To grasp the intuition behind such a behavior, the norm of the gain vector is expressed in terms of the eigenvalues $\lambda_{k}$ (arranged in decreasing order) and the corresponding eigenvectors $q_{k}$ of $\hat{\mathbf{R}}(n)$,

$$
\|\mathbf{k}(n)\|_{2}^{2}=\sum_{k=1}^{\min (m, n)}\left|\frac{\mathbf{q}_{k}^{H} \mathbf{u}(n)}{\lambda_{k}}\right|^{2} .
$$

Note that for $n<m$, the $m-n$ smallest eigenvalues of $\hat{\mathbf{R}}(n)$ are equal to $\delta$. The 
eigenvectors $\mathbf{q}_{k}$ corresponding to those "trivial" eigenvalues are orthogonal to the subspace spanned by the received snapshots $\mathbf{u}(1), \ldots, \mathbf{u}(n)$. Therefore, the summation in (4.72) goes up to index $\min (m, n)$.

As can be noted from (4.72), the norm of the gain vector is dominated by the smallest "non-trivial" eigenvalue $\lambda_{\min (m, n)}$. This eigenvalue becomes extremely small for $n \approx m$, which triggers the peak. To understand why, it is assumed that the observation vectors $\mathbf{u}(n)$ are drawn independently and uniformly from an $m$-sphere. Thus, each one contributes statistically equally to the energy along each "non-trivial" direction in the eigenspace of $\hat{\mathbf{R}}(n)$. A new non-trivial direction is acquired with each $\mathbf{u}(n)$ when $n<m$ and the energy along that direction is approximately $1 / n$ of the incoming observation vector's energy. Therefore, as $n$ approaches $m$, the energy along the newly acquired direction decreases. Consequently, the minimal non-trivial eigenvalue decreases, which causes increase in the norm of the gain vector. When $n$ exceeds $m$, $\hat{\mathbf{R}}(n)$ has full "non-trivial" rank, so each new observation vector contributes energy along all directions. The smallest eigenvalue increases, so the norm of the gain vector decreases.

To visualize how the minimal "non-trivial" eigenvalue depends on observation index $n$, the approach from [18] is adopted. Namely, the limiting eigenvalue density $\mu_{\hat{\mathbf{R}}}(x)$ is viewed as a probability density function of a randomly chosen eigenvalue. This is justified by the fact that if in a series of different realizations of $\hat{\mathbf{R}}(n)$, an eigenvalue is chosen uniformly at random for each realization, then the appropriately scaled histogram of the collection of those eigenvalues closely matches the plot of $\mu_{\hat{\mathbf{R}}}(x)$ [15]. Therefore, a cumulative distribution function of the $k$-ith largest eigenvalue $F_{k}(x)=\operatorname{Pr}\left[\lambda_{k} \leq x\right]$ is expressed as

$$
F_{k}(x)=\sum_{i=m-k+1}^{m}\left(\begin{array}{c}
m \\
i
\end{array}\right) F^{i}(x)(1-F(x))^{m-i}
$$

where $F(x)$ is a cumulative distribution function corresponding to probability density $\mu_{\hat{\mathbf{R}}}(x)$. The expected value of the smallest non-trivial eigenvalue is computed from its cumulative distribution function and plotted for different numbers of observations 


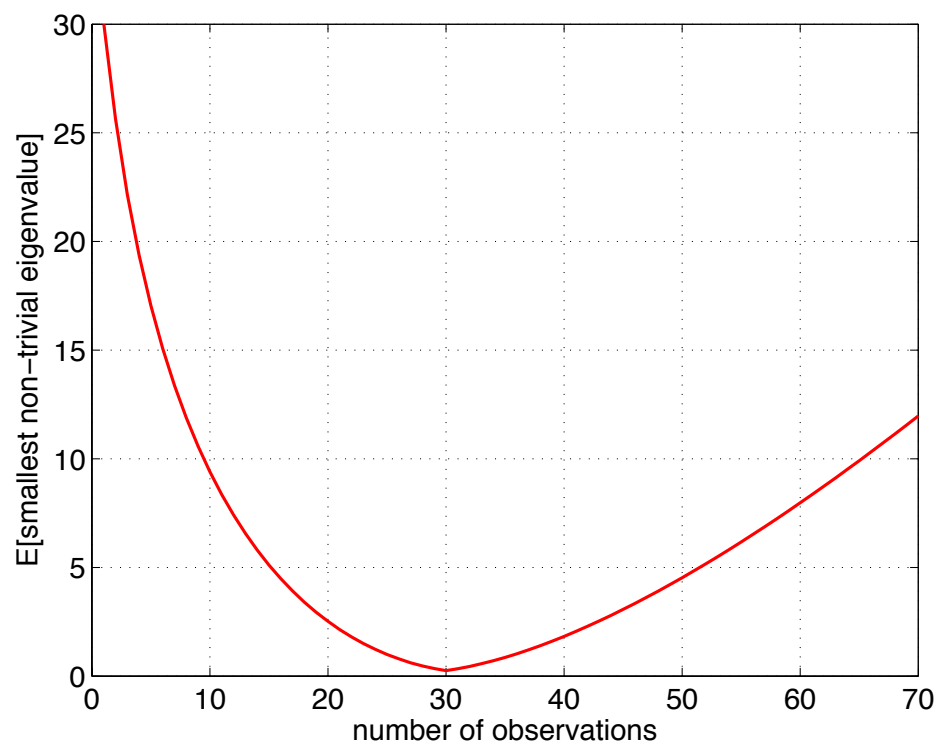

Figure 4-13: Expected values of the smallest non-trivial eigenvalue vs number of observations. The number of dimensions is 30 .

$n$ in Fig. 4-13. It can be observed that behavior of the smallest non-trivial eigenvalue is on a par with the intuitive reasoning and the plots in Fig. 4-12.

Although the norm of the gain vector $\mathbf{k}(n)$ depends only on the input process, the performance degradation for $n \approx m$ tends to appear at lower SNR's. The reason is revealed from (4.70). Namely, the channel impulse response is updated based on the gain vector $\mathbf{k}(n)$ and the signal prediction error $\xi(n)$. As SNR decreases, the signal prediction error increases. In that case, the bump in the gain vector appearing when $n \approx m$ is further amplified, causing increase in the norm of the correction term in update equation (4.70). Consequently, the channel estimation error increases. In turn, the signal prediction error at next iteration gets larger. Overall, the channel estimation error and the signal prediction error are causing each other's increase from one iteration to the next. This effect persists until the bump in the norm of the gain vector lasts.

As a final note, the SNR at which the performance degradation arises is determined by other system parameters, mainly by the channel length $m$. Following the presented intuitive reasoning, it is deduced that the largest SNR for which the performance deteriorates when $n$ is close to $m$, gets larger as the channel length increases. 


\subsection{Sliding Window RLS Algorithm}

The estimation performance of the sliding window LS algorithm is studied in this section. The analytical expression for the channel estimation MSE is validated via simulations. Also, the sliding window length which minimizes the channel estimation MSE for uncorrelated input is approximated.

\subsubsection{Performance Analysis}

The channel estimation MSE of the sliding window LS algorithm used to estimate a first order Markov channel is obtained using the developed theoretical framework. Here, we set the forgetting factor $\lambda=1$ and the number of observations $n$ is viewed as the sliding window length.

Thus, substituting $\lambda=1$ and approximations for $V_{k}$ and $W$ into (4.19), (4.22)

and (4.24), the error powers $P_{1}(n)$, due to the initial channel vector, $P_{2}(n)$, due to the channel dynamics, and $P_{3}(n)$, due to the observation noise, are evaluated in terms of the expectations of the moment, $\tilde{M}_{1}(m, n)$ and $\tilde{M}_{2}(m, n)$. Assuming $n>m$, which alleviates the need for diagonal loading $\delta$, the power of the error due to the initial channel vector is

$$
\begin{aligned}
P_{1}(n)= & m a^{2 n}-2 a^{n+1} \frac{1-a^{n}}{1-a} \frac{m}{n}+a^{2} \frac{1-a^{2 n}}{1-a^{2}} \frac{m}{n} \operatorname{tr}\{\mathbf{R}\} \tilde{M}_{1}(m, n) \\
& +\frac{2 a^{2}\left(1-a^{n}\right)\left(a-a^{n}\right) m\left(1-\tilde{M}_{1}(m, n) \operatorname{tr}\{\mathbf{R}\}\right)}{n(n-1)(1-a)^{2}(1+a)},
\end{aligned}
$$

the power of the error due to the channel dynamics is

$$
\begin{aligned}
& P_{2}(n)=m \sigma_{o}^{2} \frac{1-a^{2 n}}{1-a^{2}}-\sigma_{o}^{2} \frac{2\left(1-a^{n}\right)\left(1-a^{n+1}\right)}{(1-a)^{2}(1+a)} \frac{m}{n} \\
& +\frac{\sigma_{o}^{2}}{1-a^{2}}\left(n-a^{2} \frac{1-a^{2 n}}{1-a^{2}}\right) \frac{m}{n} \tilde{M}_{1}(m, n) \operatorname{tr}\{\mathbf{R}\} \\
& +\sigma_{o}^{2} m \frac{1-\tilde{M}_{1}(m, n) \operatorname{tr}\{\mathbf{R}\}}{n(n-1)}\left\{\frac{2 n a}{(1-a)^{2}(1+a)}-\frac{2 a\left(1-a^{n}\right)}{(1-a)^{3}}+\frac{2 a^{2}\left(1-a^{2 n}\right)}{(1-a)^{3}(1+a)^{2}}\right\}
\end{aligned}
$$


and the power of the error due to the observation noise is

$$
P_{3}(n)=m \sigma_{v}^{2}\left(\tilde{M}_{1}(m, n)-\delta \tilde{M}_{2}(m, n)\right)
$$

Overall, the channel estimation MSE is given by

$$
\mathbf{E}\left[\|\varepsilon(n)\|_{2}^{2}\right]=P_{1}(n)+P_{2}(n)+P_{3}(n) .
$$

When the ensemble correlation matrix of the input process is identity $\mathbf{I}$, the ex-

pressions (4.34) and (4.35) for moments $\tilde{M}_{1}$ and $\tilde{M}_{2}$ are used to evaluate $P_{1}, P_{2}$ and $P_{3}$. Also in that case a signal prediction MSE is approximated with (4.28). On the other hand, when the input observations are of an unconstrained correlation $\mathbf{R}$, $\tilde{M}_{1}(n, m)$ is evaluated for $n>m$ using (4.33).

\subsubsection{Theoretical versus Numerical Results}

The accuracy of the derived expressions is tested using Monte-Carlo simulations. When $\mathbf{R}=\mathbf{I}$, the corresponding plots for a slowly varying channel $(a=0.995)$ are shown in Fig. 4-14 and 4-15. When the channel varies more rapidly, i.e., for $a=0.95$, the corresponding plots for the channel estimation and signal prediction errors are shown in Fig. 4-16 and Fig. 4-17.

The corresponding comparisons between the theory and simulations when an input has an unconstrained correlation $\mathbf{R}$ are shown in Fig. 4-18 and Fig. 4-19 for respectively slowly $(a=0.995)$ and more rapidly $(a=0.95)$ varying channel.

In all cases, the input process is Gaussian distributed, channel has length $m=30$, process noise is of variance $\sigma_{o}^{2}=0.01$ and SNR is $10 \mathrm{~dB}$. As a technical detail, the SNR is defined at the channel output. Since the input signal's power per channel tap is $\operatorname{tr}\{\mathbf{R}\} / m$ and the expected squared L2 norm of the channel vector in steady state is $\left(m \sigma_{o}^{2}\right) /\left(1-a^{2}\right)$, the SNR is given by

$$
\mathrm{SNR}=10 \log _{10} \frac{\sigma_{o}^{2} \operatorname{tr}\{\mathbf{R}\}}{\sigma_{v}^{2}\left(1-a^{2}\right)}
$$


The vertical axis in each plot is normalized with the L2 norm of the channel vector in the steady state.

Note that as the window length $n \rightarrow \infty$, the estimation MSE asymptotically approaches $0 \mathrm{~dB}$ level. This is because all the channel variations are averaged out with infinitely large rectangular window so that the estimated channel vector approaches 0. Therefore, the estimation error converges to the L2 norm of the channel vector (i.e., to $0 \mathrm{~dB}$ in figures due to normalization).

For a finite averaging window length $n$, the performance curves exhibit different behaviors depending on the value of the state transition parameter $a$. As such, when the channel varies rapidly, the performance curve is above $0 \mathrm{~dB}$ level as can be observed in Figures 4-16, 4-17 and 4-19, where $a=0.95$. Effectively, the RLS algorithm is not able to track such a channel and the channel estimation error is minimized for a trivial estimator $\hat{\mathbf{w}}=\mathbf{0}$. On the other hand, when the channel varies slowly, the optimal channel estimation MSE is below $0 \mathrm{~dB}$ level and is achieved for a finite averaging window length. This could be observed in Figures 4-14, 4-15 and 4-18 where $a=0.995$.

Furthermore, it could be noted that the performance curves corresponding to smaller $a$ shown in Figures 4-16 and 4-17 show the counterintuitive result that when the number of observations drops below the dimensionality of the system, there is a drop in the MSE as the number of observations is decreased. This result is not due to the tracking of channel dynamics as is the case in the downward trend in MSE as the number of observations is decreased and has been studied and explained in Section 4.5.3.

Finally, recall that the characterization of signal prediction MSE has been derived by assuming that the state transition parameter $a$ is close to 1 . Besides obtaining a good agreement between the theoretical predication and simulations of the signal prediction MSE corresponding to a slowly varying channel when $a=0.995$ (not shown in figures), a good agreement is obtained even for a rapidly varying channel when $a=0.95$, shown in Fig. 4-17. Although this latter case does not have practical significance because the RLS algorithm is not able to track the channel reasonably 


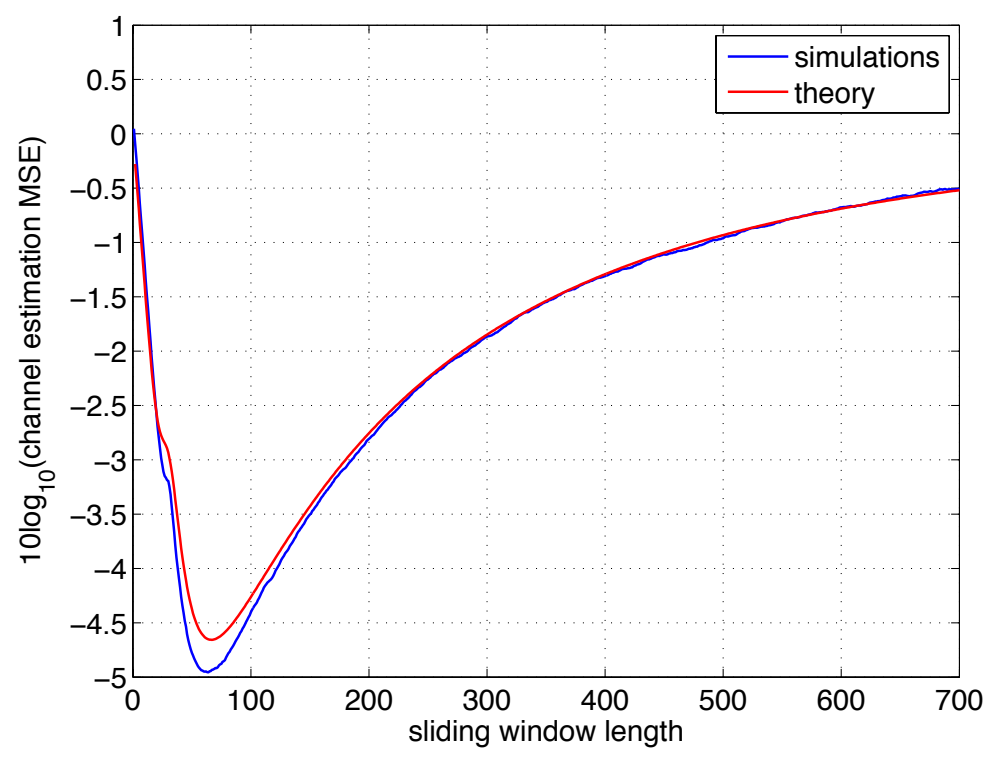

Figure 4-14: Theoretical versus simulated channel estimation MSE for $\mathrm{a}=0.995, \sigma_{o}=$ 0.1 and $\mathrm{SNR}=10 \mathrm{~dB}$.

well, it confirms that the derived characterization is valid at least for the range of $a$ 's corresponding to the cases of practical significance.

\subsubsection{Optimal Window Length}

An approximate expression for the optimal window length $n_{\text {opt }}$ is derived in this part using the observation that the RLS algorithm reasonably well tracks the channel when the state transition parameter $a$ is close to 1 . Therefore, we assume that the channel vector exhibits a random walk (i.e., $a \rightarrow 1$ ). In that case the steady state mean square channel estimation error for a sliding window length $n>m$ is obtained from (4.77) by letting $a \rightarrow 1$ and $\delta \approx 0$ as

$$
\mathbf{E}\left[\|\varepsilon(n)\|_{2}^{2}\right]=\sigma_{o}^{2} \frac{m\left(2 n^{2}-n m+5 m-4 n\right)}{6(n-m)}+\sigma_{v}^{2} \frac{m}{n-m} .
$$

Setting the first derivative of (4.79) to zero (or equating the error terms induced by the channel dynamics and observation noise) and solving for $n$, yields the expression 


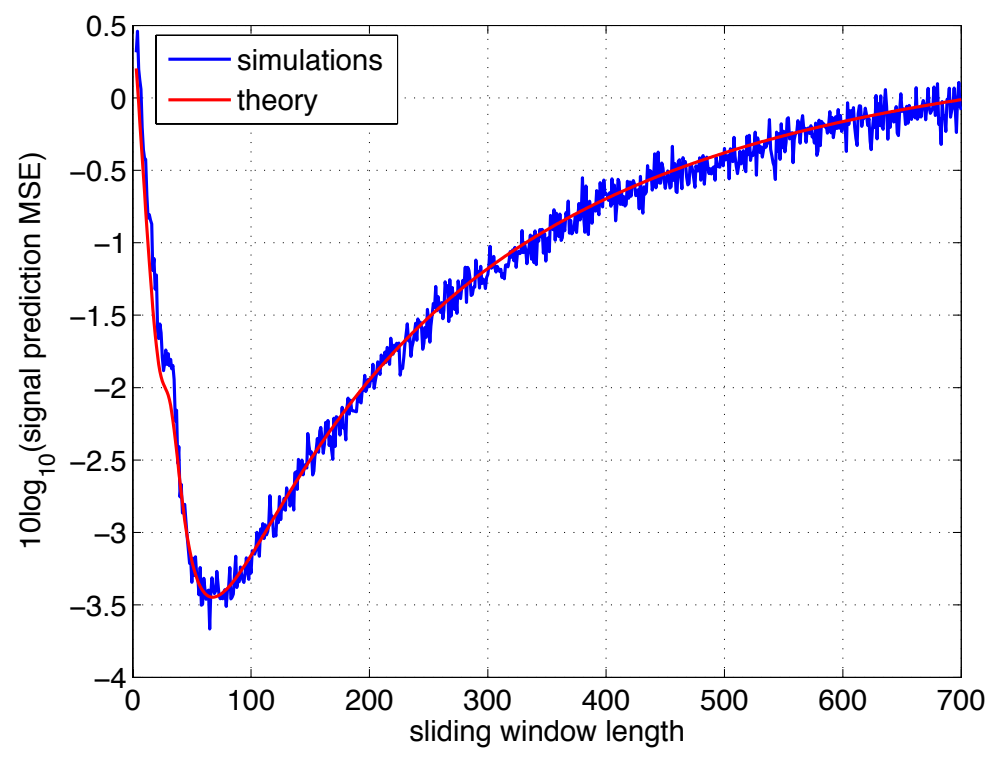

Figure 4-15: Theoretical versus simulated signal prediction MSE for $\mathrm{a}=0.995, \sigma_{o}=0.1$ and $\mathrm{SNR}=10 \mathrm{~dB}$.

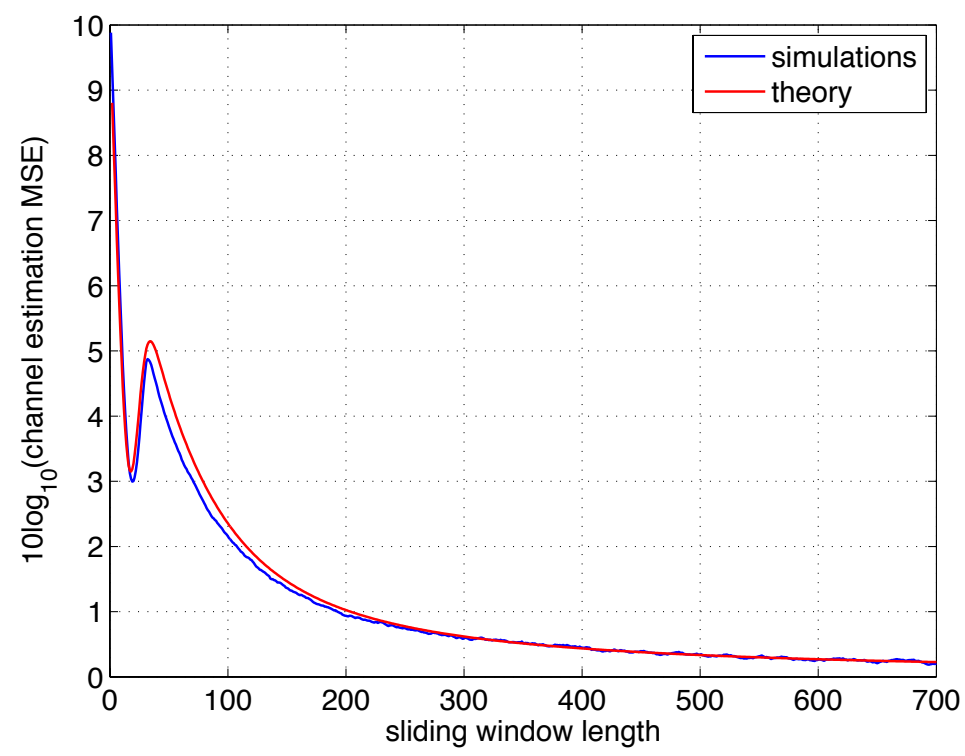

Figure 4-16: Theoretical versus simulated channel estimation MSE for a $=0.95, \sigma_{o}=$ 0.1 and $\mathrm{SNR}=10 \mathrm{~dB}$. 


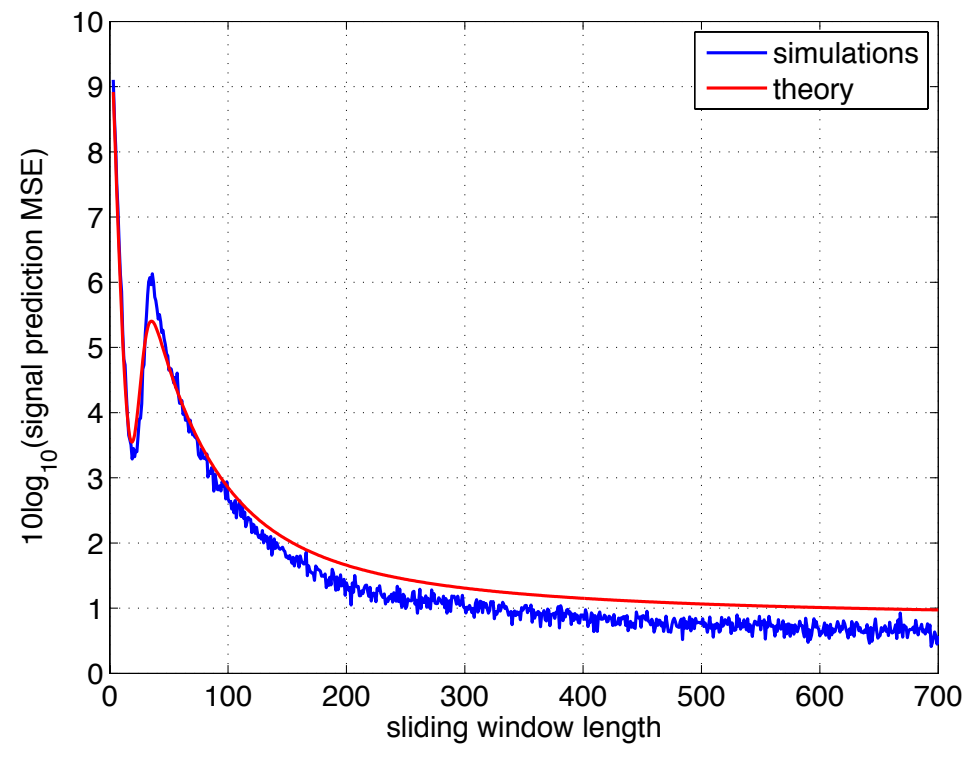

Figure 4-17: Theoretical versus simulated signal prediction MSE for $\mathrm{a}=0.95, \sigma_{o}=0.1$ and $\mathrm{SNR}=10 \mathrm{~dB}$.

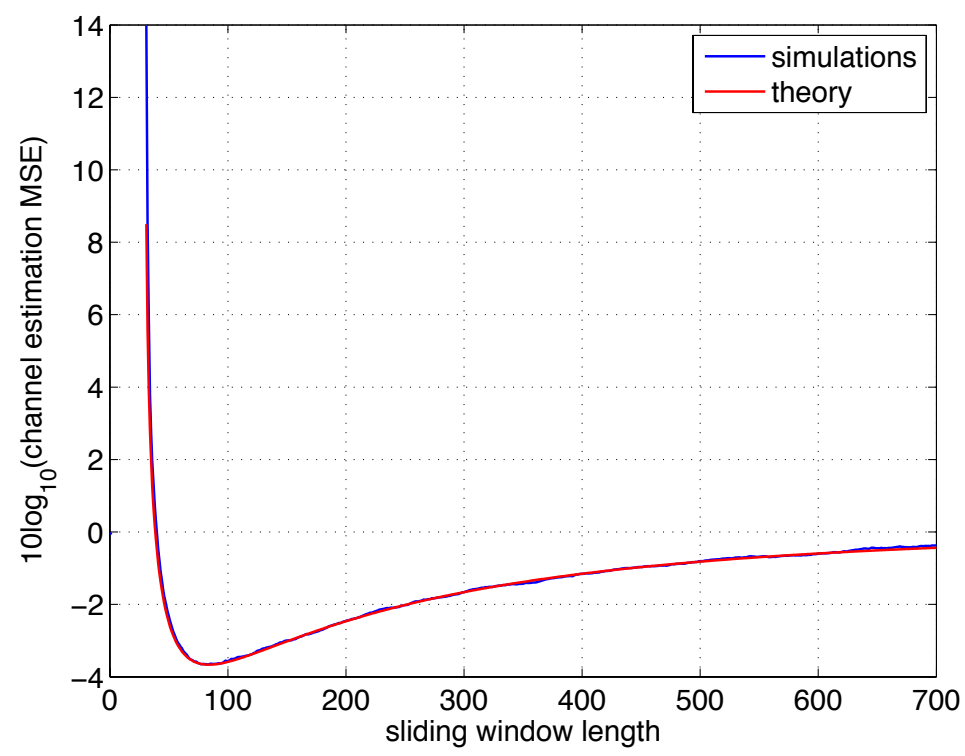

Figure 4-18: Theoretical versus simulated channel estimation MSE for a $=0.995, \sigma_{o}=$ $0.1, \mathrm{SNR}=10 \mathrm{~dB}$, and input process of unconstrained correlation. 


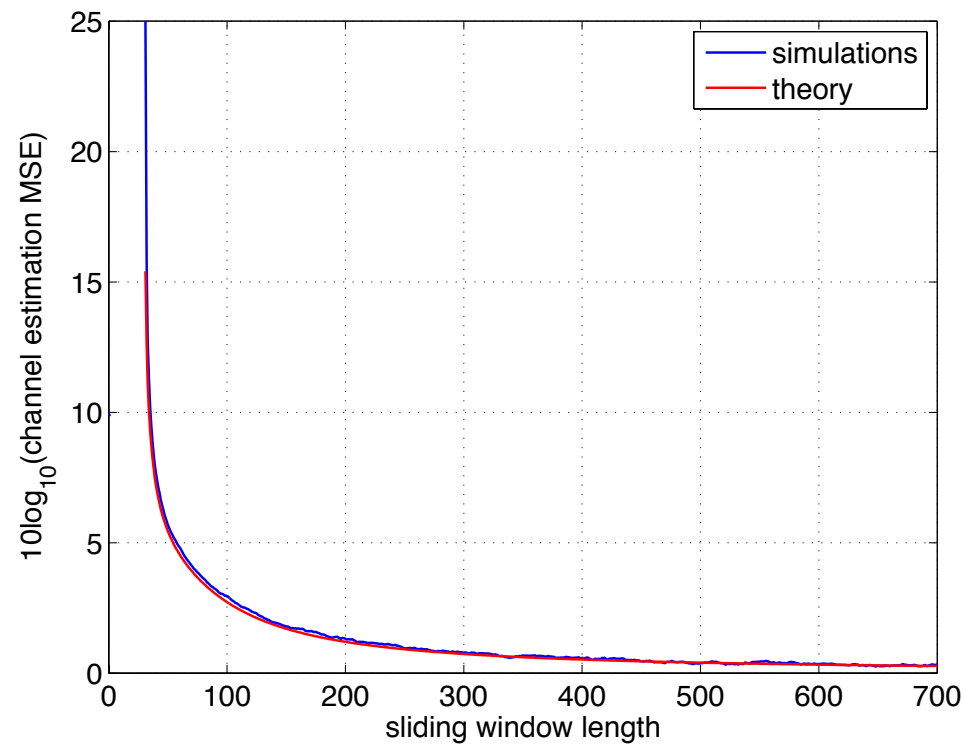

Figure 4-19: Theoretical versus simulated channel estimation MSE for a $=0.95, \sigma_{o}=$ $0.1, \mathrm{SNR}=10 \mathrm{~dB}$, and input process of unconstrained correlation.

for the optimal sliding window length. When $\mathbf{R}=\mathbf{I}$, this expression simplifies to

$$
n_{\mathrm{opt}}=m+\sqrt{\frac{m^{2}+m+6 \frac{\sigma_{v}^{2}}{\sigma_{o}^{2}}}{2}} .
$$

The accuracy of (4.80) is tested via simulations for different values of $a$, SNR and process noise variance $\sigma_{o}^{2}$, and the results are summarized in Table 4.1. The entries $n_{\mathrm{opt}}$ and $n_{\mathrm{opt}}^{\text {sim }}$ pertain to respectively (4.80) and the simulated optimal window length. The quantity $\Delta$ is the difference between the simulated MSE's corresponding to window lengths $n_{\mathrm{opt}}$ and $n_{\mathrm{opt}}^{\mathrm{sim}}$. The channel length in all considered cases is 30 . As can be observed from the table, the error due to using $n_{\mathrm{opt}}$ evaluated form (4.80) does not exceed $0.05 \mathrm{~dB}$.

Finally, note that the outlined method for finding the optimal sliding window length is derived by assuming a random walk model for the channel. The simulation results in Table 4.1 imply that the expression is fairly accurate when channel varies according to a first order Markov process whose state transition parameter $a$ is close to 1. However, this is in fact the regime of practical importance because the estimation 
Table 4.1: Optimal Sliding Window Length

\begin{tabular}{|c|c|c|c|c|r|}
\hline$a$ & $\sigma_{o}^{2}$ & SNR & $n_{\text {opt }}$ & $n_{\text {opt }}^{\text {sim }}$ & $\Delta$ \\
\hline 1 & 0.01 & $10 \mathrm{~dB}$ & 52 & 48 & $=0.06 \mathrm{~dB}$ \\
\hline 0.99 & 0.01 & $10 \mathrm{~dB}$ & 60 & 63 & $<0.05 \mathrm{~dB}$ \\
\hline 0.98 & 0.01 & $10 \mathrm{~dB}$ & 56 & 65 & $<0.05 \mathrm{~dB}$ \\
\hline 0.99 & 0.01 & $20 \mathrm{~dB}$ & 50 & 53 & $=0.006 \mathrm{~dB}$ \\
\hline 0.98 & 0.0001 & $20 \mathrm{~dB}$ & 52 & 58 & $=0.017 \mathrm{~dB}$ \\
\hline 0.99 & 0.01 & $5 \mathrm{~dB}$ & 74 & 77 & $=0.027 \mathrm{~dB}$ \\
\hline
\end{tabular}

error when a state transition parameter $a$ is not sufficiently close to 1 is relatively high for any finite averaging window length, implying that the channel is effectively not being tracked.

\subsection{Insights in Exponentially Weighted and Slid- ing Window LS Algorithms}

This section develops some practical results about the LS algorithm when the input process has identity correlation. These results are valid even if the input process has few distinct eigenvalues. Namely, it has been observed that the eigenvalues corresponding to the noise-only subspace of the input process which has few distinct eigenvalues, approximately behave as if the input process has identity correlation. This qualitative observation has also been exploited to develop algorithms that estimate the number of signals embedded into the noise with small number of samples [30].

\subsubsection{Effective Number of Observations}

When the input observations are exponentially windowed with forgetting factor $\lambda$, it is often of practical importance to assess the equivalent number of stationary, rectangulary windowed observation vectors that give rise to the same quality of the SCM. A commonly used rule of thumb is $n_{\mathrm{eff}}=1 /(1-\lambda)$. A more accurate relation is derived in this part using random matrix theory results. 
Formally, the problem can be formulated as finding $n_{\text {eff }}$ such that

$$
\lim _{n \rightarrow \infty} \sum_{k=1}^{n} \lambda^{n-k} \mathbf{u}_{L}(k) \mathbf{u}_{L}^{H}(k) \approx \sum_{k=1}^{n_{\mathrm{eff}}} \mathbf{u}_{R}(k) \mathbf{u}_{R}^{H}(k),
$$

where the vectors $\mathbf{u}_{L}(k)$ and $\mathbf{u}_{R}(n)$ of length $m$ originate from the identical and stationary processes of zero mean and identity correlation.

The SCM's on the left and right hand sides of (4.81) are denoted respectively $\boldsymbol{\Phi}_{L}$ and $\boldsymbol{\Phi}_{R}$. The limiting Eigenvalue Density Function $\mu_{L}(x)$ of $\boldsymbol{\Phi}_{L}$ is parameterized with $q=m(1-\lambda)$ and is given by (2.63) and (2.4). The limiting Eigenvalue Density Function $\mu_{L}(x)$ has a compact support whose endpoints $x_{1}$ and $x_{2}$ are given by

$$
x_{1,2}=\log x_{1,2}+q+1 .
$$

The limiting Eigenvalue Density Function $\mu_{R}$ of $\boldsymbol{\Phi}_{R}$ is given by Marcenko-Pastur law (2.56) and is parameterized with $c=\frac{m}{n_{\mathrm{eff}}}$. The endpoints of the support are for $c<1$ given by

$$
\begin{aligned}
& l_{\min }=(1-\sqrt{c})^{2}, \\
& l_{\max }=(1+\sqrt{c})^{2} .
\end{aligned}
$$

Since the plots of $\mu_{L}$ and $\mu_{R}$ look alike when $c<1$ (because then $\mu_{R}$ has no mass at 0 ), equation (4.81) is approximately solved by matching the endpoints of the two density functions. Thus, the effective number of observations $n_{\mathrm{eff}}$ for which the upper limits $x_{1}$ and $l_{\max }$ of $\mu_{L}$ and $\mu_{R}$ coincide is from (4.82) and (4.84) given by

$$
(1+\sqrt{c})^{2}=2 \log (1+\sqrt{c})+q+1
$$

where $c=\frac{m}{n_{\text {eff }}}$. A requirement $c<1$ is equivalent to $q<1.6$. A similar equation can be obtained for the lower limits to coincide.

To derive a more handy relation between $\lambda$ and $n_{\text {eff }}$, the first two terms of the Taylor series expansion of $\log$ function (the series exists because $c<1$ ) are taken into 


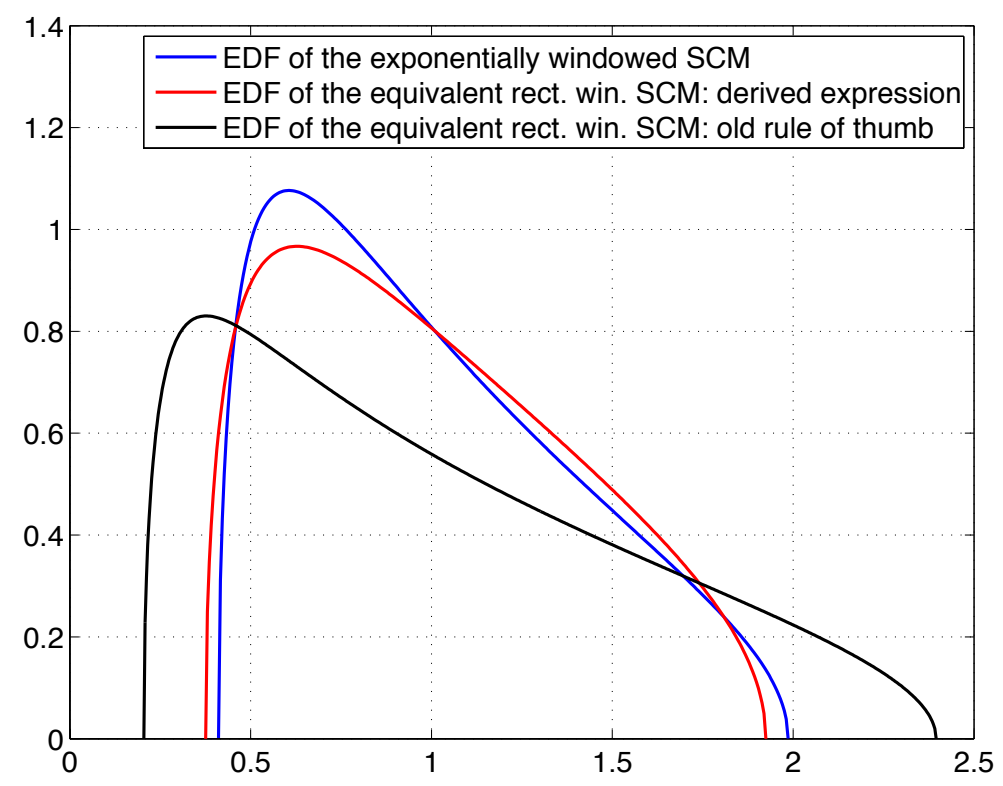

Figure 4-20: The limiting Eigenvalue Density Functions of the exponentially weighted SCM and SCM's with rectangular windowing with the effective observation window size computed from the derived expression (4.86) and conventional rule of thumb $1 /(1-\lambda)$. The dimension of the observation vector is $m=30$, forgetting factor is $\lambda=0.99$ and $q=0.3$.

account, which yields

$$
n_{\mathrm{eff}}=\frac{2}{1-\lambda}
$$

The same expression is obtained if the lower limits $x_{2}$ and $l_{\min }$ are matched and the $\log$ function is approximated with first two terms of its Taylor series.

The relation (4.86) is tested by comparing the plots of the limiting EDF's $\mu_{L}$ and $\mu_{R}$ for $\lambda=0.99$ and $\lambda=0.95$ in Fig. 4-20 and Fig. 4-21, respectively. The limiting EDF's corresponding to the SCM with rectangular windowing whose observation window size is computed using the conventional rule of thumb $1 /(1-\lambda)$ are also shown. The problem dimension in both cases is $m=30$. Note that parameter $q$ corresponding to Fig. 4-21 is 1.5, which is close to the value (of 1.6) for which a resemblance between the two plots is possible. Also, since (4.86) is an approximation of (4.85), the endpoints of the two density functions do not coincide exactly. 


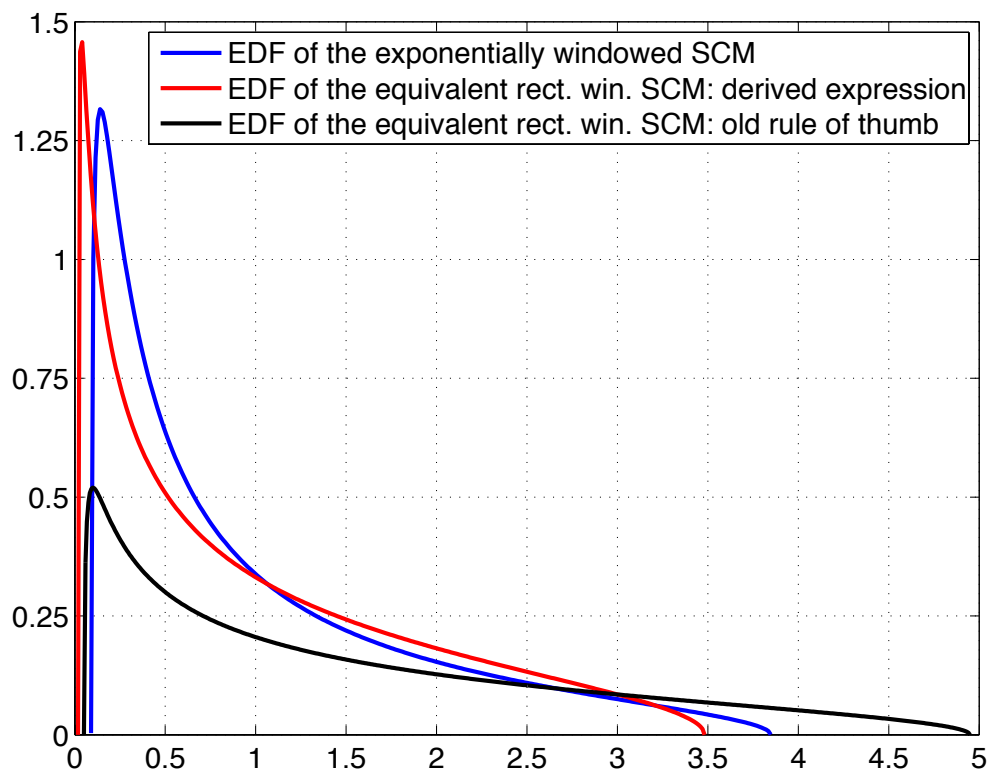

Figure 4-21: The limiting Eigenvalue Density Functions of the exponentially weighted SCM and SCM's with rectangular windowing with the effective observation window size computed from the derived expression (4.86) and conventional rule of thumb $1 /(1-\lambda)$. The dimension of the observation vector is $m=30$, forgetting factor is $\lambda=0.95$ and $q=1.5$ 


\subsubsection{Exponentially Weighted versus Sliding Window RLS}

The LS algorithm suffers from the performance deterioration if the SCM is ill-conditioned. The appearance of a bump in the channel estimation and signal prediction MSE's, described for the LTI channel identification case in Section 4.5.3, is intrinsically caused by ill-conditioned SCM. The exponentially weighted and sliding window LS algorithms are compared based on how well the corresponding SCM's are conditioned. Namely, comparing the plots of the limiting eigenvalue density functions from Figures 4-20 and 4-21, one may observe that the Eigenvalue Density Function of the rectangularly windowed SCM tends to have eigenvalues closer to zero. In other words, it is easier to bound the eigenvalues away from zero in the exponentially windowed algorithm than in its rectangularly windowed counterpart. Consequently, it can be conjectured that the exponential weighting with forgetting factor $\lambda$ could be better suited than the rectangular windowing with window length $n$.

More specifically, given the sliding window length $n$, it is possible to determine an equivalent forgetting factor $\lambda$ such that the upper end points of the limiting eigenvalue densities of the corresponding SCM's coincide. Consequently, the lower end point of the density pertaining to the exponentially weighted SCM is slightly further away from the origin meaning that the SCM is better conditioned. For a given sliding window length $n$, the corresponding $\lambda$ which ensures this is using (4.85), given by

$$
\lambda=1-\frac{\left(1+\sqrt{\frac{m}{n}}\right)^{2}-2 \log \left(1+\sqrt{\frac{m}{n}}\right)-1}{m} .
$$

The behaviors of the sliding window LS with the window length $n$ and the LS with the exponentially weighted window with forgetting factor $\lambda$, computed using (4.87), are compared via simulations. The simulation study shows that the exponentially weighted LS outperforms the sliding window LS for both LTI and first order Markov channels. Fig. 4-22 and Fig. 4-23 show the performance plots of the considered algorithms. It can be conjectured that given the window length $n$ in a sliding window RLS, it is always possible to compute forgetting factor $\lambda$ using (4.87), such that the exponentially weighted RLS outperforms a sliding window RLS in a steady state. 


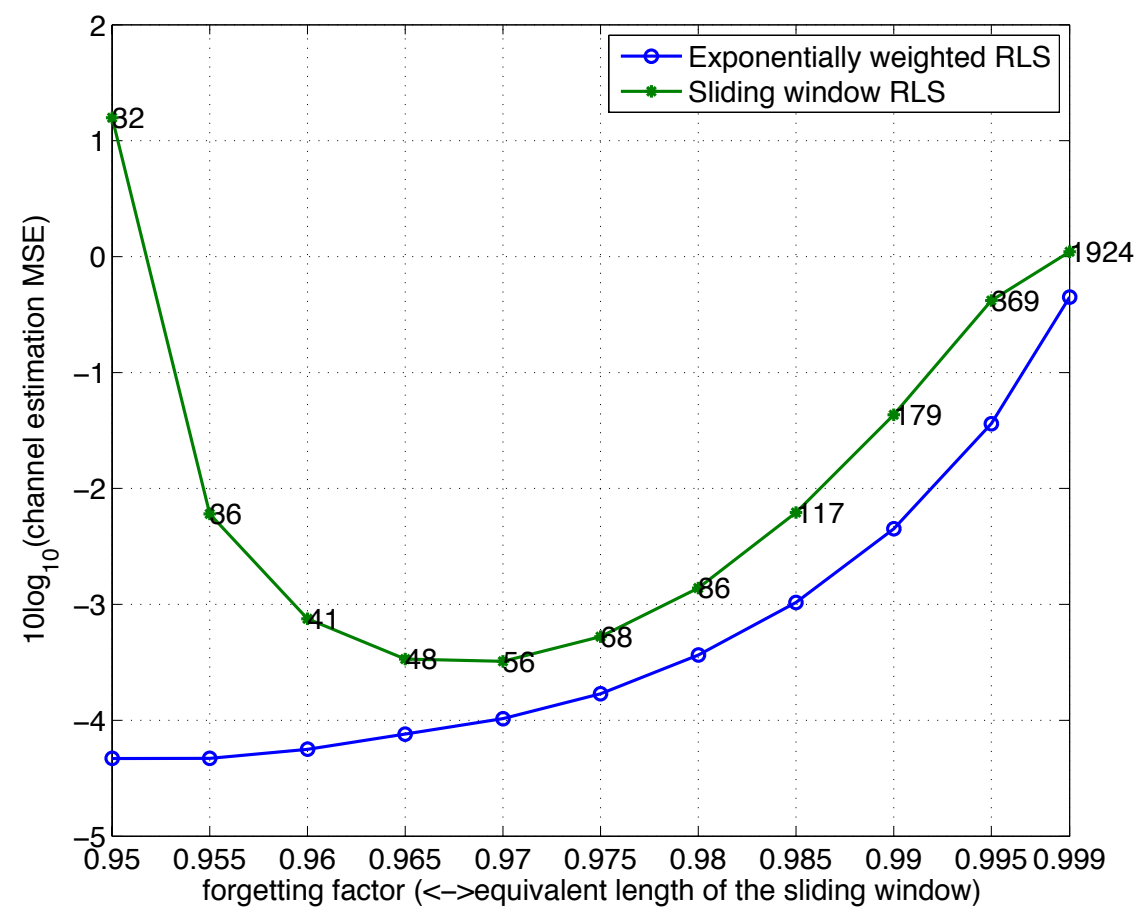

Figure 4-22: Exponentially weighted versus sliding window RLS for SNR=10 dB. The integers along the green curve represent the sliding window lengths equivalent to the corresponding values of forgetting factor.

Furthermore, at low SNR's, the optimal window length of the sliding window RLS and the optimal forgetting factor of an exponentially weighted RLS are related as in (4.87). At higher SNR's, the optimal forgetting factor is slightly below the value computed using (4.87) for a given $n=n_{\mathrm{opt}}$. However, the difference between the MSE's at optimal forgetting factor and that at $\lambda$ corresponding to the optimal sliding window length $n_{\text {opt }}$ when SNR is high is negligible.

\subsubsection{Optimal Value of Forgetting Factor}

The optimal $\lambda$ for the exponentially weighted LS algorithm is approximated by using the expression for the optimal window length of the sliding window RLS algorithm (4.80) and the relationship between $\lambda$ and the effective number of observations $n$ in the rectangularly windowed SCM (4.86). The justification for such an approach is the observation that even at large SNR's, the difference between the MSE's at 


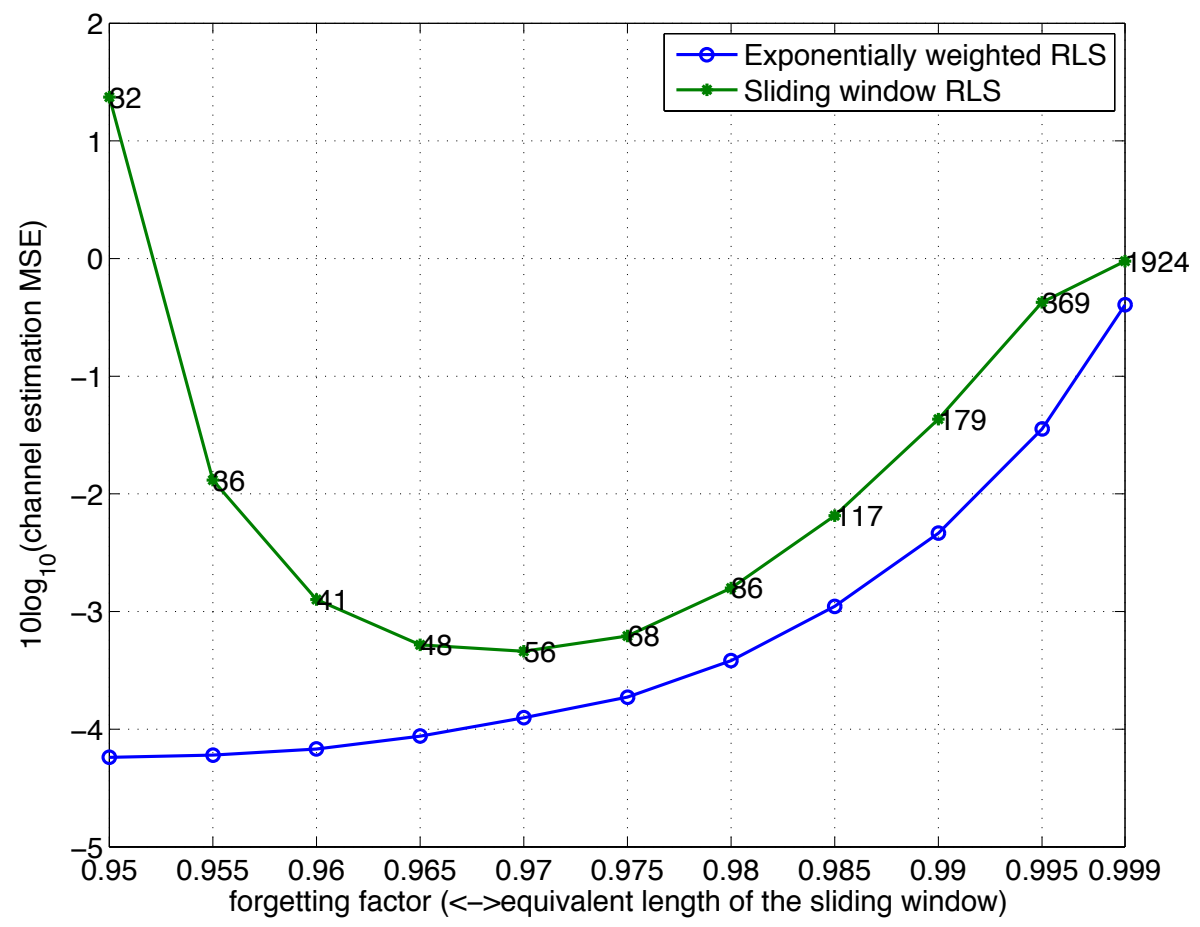

Figure 4-23: Exponentially weighted versus sliding window RLS for SNR=5 dB. The integers along the green curve represent the sliding window lengths equivalent to the corresponding values of forgetting factor. 
Table 4.2: Optimal Forgetting Factor Value

\begin{tabular}{|c|c|c|c||c|c||c|c|}
\hline$a$ & $\sigma_{o}^{2}$ & $\mathrm{SNR}$ & $\lambda_{\text {opt }}^{\text {sim }}$ & $\lambda_{\text {opt }}^{(1)}$ & $\Delta_{1}[\mathrm{~dB}]$ & $\lambda_{\text {opt }}^{(2)}$ & $\Delta_{2}[\mathrm{~dB}]$ \\
\hline 0.99 & 0.01 & $5 \mathrm{~dB}$ & 0.975 & 0.973 & 0.045 & 0.977 & 0.04 \\
\hline 0.99 & 0.01 & $10 \mathrm{~dB}$ & 0.960 & 0.967 & 0.065 & 0.972 & 0.155 \\
\hline 0.99 & 0.01 & $20 \mathrm{~dB}$ & 0.949 & 0.962 & 0.153 & 0.968 & 0.309 \\
\hline 0.99 & 0.01 & $40 \mathrm{~dB}$ & 0.942 & 0.962 & 0.235 & 0.968 & 0.375 \\
\hline 0.98 & 1 & $5 \mathrm{~dB}$ & 0.980 & 0.969 & 0.107 & 0.973 & 0.075 \\
\hline 0.98 & 1 & $10 \mathrm{~dB}$ & 0.969 & 0.964 & 0.074 & 0.970 & 0.019 \\
\hline 0.98 & 1 & $20 \mathrm{~dB}$ & 0.953 & 0.961 & 0.037 & 0.968 & 0.108 \\
\hline 0.98 & 1 & $40 \mathrm{~dB}$ & 0.956 & 0.961 & 0.048 & 0.968 & 0.137 \\
\hline
\end{tabular}

optimal forgetting factor and that at $\lambda$ corresponding to the optimal sliding window length $n_{\text {opt }}$ is negligible. Overall from (4.80) and (4.86),

$$
\lambda_{o p t}^{(1)}=1-\frac{2}{m+\sqrt{\frac{m^{2}+m+6 \frac{\sigma_{v}^{2}}{\sigma_{0}^{2}}}{2}}} .
$$

A potentially more accurate expression for the optimal forgetting factor $\lambda_{\text {opt }}^{(2)}$ is obtained by substituting $n=n_{\text {opt }}$, evaluated using (4.80), into the exact expres$\operatorname{sion}(4.87)$.

The derived expressions are tested by comparing $\lambda_{\text {opt }}^{(1)}$ and $\lambda_{\text {opt }}^{(2)}$ with the optimal forgetting factor obtained via simulations for given system parameters. The results are summarized in Table 4.2. The channel length in all cases is 30. The quantities $\Delta_{1}$ and $\Delta_{2}$ represent the performance loss due to respectively choosing theoretically calculated values $\lambda_{\text {opt }}^{(1)}$ and $\lambda_{\text {opt }}^{(2)}$ instead of $\lambda_{\text {opt }}^{\text {sim }}$. The optimal forgetting factor (4.9) from [27] is also tested and it fails to yield accurate result (in fact, it yields too small values for $\lambda$ ), which comes at no surprise since it is derived under the assumption that both $\lambda$ and $a$ are very close to 1 . As can be observed from the table, the performance loss due to using optimal forgetting factor proposed here does not exceed $0.5 \mathrm{~dB}$.

Overall, for relatively small SNR, both expressions give accurate results. On the other hand, when SNR is high, the optimal forgetting factor is below that computed using (4.87), (refer to Fig. 4-22). In that case, $\lambda_{\text {opt }}^{(1)}$ is more accurate because it is always smaller than $\lambda_{\text {opt }}^{(2)}$ and hence closer to the true optimal value. 
As a final remark, note that although the characterization of optimal forgetting factor is derived for $a \rightarrow 1$, the simulations show that it is fairly accurate for the values of $a$ smaller than 1 for which the RLS algorithm reasonably well tracks the corresponding channel.

\subsection{Conclusions}

An analysis of the Least Squares algorithm when employed to track a time-varying channel is performed using results and tools from the theory of large dimensional random matrices. A time-varying channel is modeled as a first order Markov process and the performance metrics of interest are mean square values of the channel estimation and signal prediction errors. These metrics are characterized for a given number of observations, channel length and the parameters describing the channel dynamics. The results borrowed from the random matrix theory enable the analysis which does not rely on the direct averaging assumption and the assumption that the expectation of the inverse of the sample correlation matrix is a scaled inverse of the ensemble correlation matrix.

The simulation study validates the derived analytical expressions. In addition, several practical results are revealed by specifying the general theory for simpler cases. First, an expression for the optimal window length in the sliding window LS algorithm is derived. Second, based on the comparison between the exponentially weighted and sliding window LS algorithms, it is conjectured that former outperforms the latter, if forgetting factor is appropriately selected given the sliding window length. The corresponding expression for such a forgetting factor is derived. Third, a relation between forgetting factor used for calculating an exponentially weighted sample correlation matrix and the effective number of stationary, rectangularly windowed observations is established. Fourth, this relation is further exploited to evaluate the optimal value of the forgetting factor in the exponentially weighted LS algorithm. Finally, the effect of performance deterioration appearing when the number of observations is close to channel length (i.e., the number of dimensions) is observed, theoretically analyzed 
and intuitively elaborated. 


\section{Chapter 5}

\section{Channel Equalization}

\section{$5.1 \quad$ Introduction}

The wireless communication channels through which signals are transmitted are often time-varying and characterized by multipath propagation. The multipath propagation gives rise to a delay spread, resulting in intersymbol interference (ISI) in the received signal, while the time-variability results in the Doppler spreading of the signal [49]. These effects are even more profound in the setting of underwater acoustic communications, wherein along with a high latency appearing due to a relatively slow speed of propagation (nominally $1500 \mathrm{~m} / \mathrm{s}$ ), and frequency dependent attenuation of the transmitted signal, these effects pose significant challenges to communication system design [51].

Different techniques have been developed for mitigating these effects [50]. A survey of the approaches used for the underwater acoustic communication system design is given in [48]. Most techniques rely in part or completely on channel equalization with a Decision Feedback Equalizer (DFE) being the most commonly used form [39]. A multi-channel DFE (MC-DFE) is one which processes the signals received at multiple spatially separated sensors. The MC-DFE is particularly effective at compensating for the ISI induced by the multipath commonly present in the underwater acoustic communication channel.

One of the main challenges in optimally configuring a multi-channel equalizer is 
the choice of the number of sensors and length of the constituent filters. Namely, due to channel time variability, the number of coefficients that can be adapted over the time interval within which the channel is approximately time invariant is limited such that a smaller number of coefficients might lead to better equalization performance.

Another challenge related to optimally configuring the mutli-channel equalizer is the selection of the separation between sensors. While the sensors in a multiple input multiple output (MIMO) system need to be sufficiently apart so that the signals at their outputs are uncorrelated [20], conventional wisdom is that array processing applications require that sensors be separated by no more than one half the shortest wavelength of the received signals [52]. However, because an inherently wideband signal is transmitted through a sparse underwater acoustic communication channel, selection of optimal sensor separation is a more subtle problem.

Although equalizers have been in common use for a while, a great deal of what is currently known is learned from simulations and processing of experimental data, while the analytical results appeared relatively recently. As such, [62] studies the signal-to-noise-puls-interference ratio (SINR) at the output of the LS-based linear equalizer for a time invariant frequency flat fading channel. A more general analysis in [38] characterizes the SINR at the output of the LS-based linear equalizer for time invariant frequency selective channels. Both works exploit random matrix theory results which, as elaborated in Section 2.7, while theoretically valid in the limit, are fairly accurate in modeling equalizer performance in practical finite observation time scenarios. In terms of optimal DFE design, [23] and [24] discuss how the decision delay, feed-forward and feedback filter lengths should be selected such that the signal prediction MSE of the MMSE based DFE equalizer of an LTI channel is minimized. However, these works inherently assume that the observation period is infinitely long, which is not the case in non-stationary channels where only a limited number of stationary observations are available for adaptation.

The contributions of this chapter are threefold. First, a performance of least squares (LS)-based MC-DFE equalization method is theoretically analyzed. The case of linear equalization is included as a special case. The transmission channel is non- 
stationary and modeled as a frequency selective filter which is time-invariant over only short time intervals. A signal prediction error is adopted as the performance metric and characterization of its mean square value is derived. In comparison to [38], in addition to handling the case of DFE equalization and evaluating the signal prediction MSE, the analysis technique is different and yields a greatly simplified closed-form result, which is exact when the received signal is Gaussian distributed. The derived expression is validated via Monte-Carlo simulations.

The derived expressions quantitatively support the observed performance characteristic that, when working with signals that have passed through time-varying channels, an equalizer with relatively short constituent filters can outperform one using longer filters [40]. The optimal number of taps in the equalizer's constituent filters is presented as a trade-off between two competing requirements. On one hand, for a perfectly known environment, the MMSE error criterion is a non-increasing function of filter length. On the other hand, the insights from random matrix theory imply that for a given number of observation vectors, the shorter constituent filters lead to more accurate estimate of the correlation matrix and therefore improved performance.

Finally, the chapter analyzes how the number of and separation between the sensors impacts the equalization performance in a time-varying underwater acoustic communication channel. Our model for the arrival process takes into account that the underwater acoustic communication signal received on an array of sensors is wideband and spatially spread. The signal prediction mean square error (MSE) is evaluated using this model. An illustration of how the equalization performance depends on the number of sensors and their separation for a particular arrival model is then provided. Finally, the bit error rate (BER) and signal prediction MSE performance, obtained from processing experimental data using a multi-channel equalizer with different sensor separations, are presented. This justifies the conclusion that the equalization performance is optimized for a non-trivial sensor separation.

Although the problems considered in this chapter are mainly motivated by underwater acoustic communications, the developed theoretical results and insights are also applicable to other settings. As such, the performance analysis of time varying 
channel equalization is general and the insights hold for other applications of least squares based equalizers. Furthermore, the study of the impact of sensor separation on the performance of multi-channel equalization is, for example, applicable to the contexts of increasingly popular $60 \mathrm{GHz}$ ultra-wideband communications [64] and optimal receiver design based on massive MIMO [31].

The rest of the chapter is organized as follows. A background on the MC-DFE which describes its structure, analytical framework and updating algorithm is given in Section 5.2. Section 5.3 presents the performance analysis of the MC-DFE when adapting using a limited number of observations of the received signal. Section 5.4 argues that the optimal number of coefficients which optimize the equalization performance is a trade off between two competing requirements. The model for sparse, wideband and spatially spread arrivals, inherent to the underwater acoustic environment is presented in 5.5. The impact of sensor separation and array aperture on performance of multi-channel equalizer is studied in Section 5.6. Finally, Section 5.7 concludes the chapter.

\subsection{Background}

The structure of the multi-channel decision feedback equalizer (MC-DFE), analytical framework and least squares based adaptation algorithm are briefly described in this section.

\subsubsection{MC-DFE: Structure}

The structure of the MC-DFE equalizer is shown in Fig. 5-1. It contains a feedforward (FF) filter bank, feedback (FB) filter and a decision device. The FF filter bank consists of one linear filter to process the input from each channel (sensor). A signal received at each sensor is after some pre-processing (such as conversion to the baseband) processed by a corresponding FF filter. The ultimate goal of the FF processing is to coherently combine the received signal energy and attenuate intersymbol interference (ISI) and ambient noise signals. On the other hand, the linear 


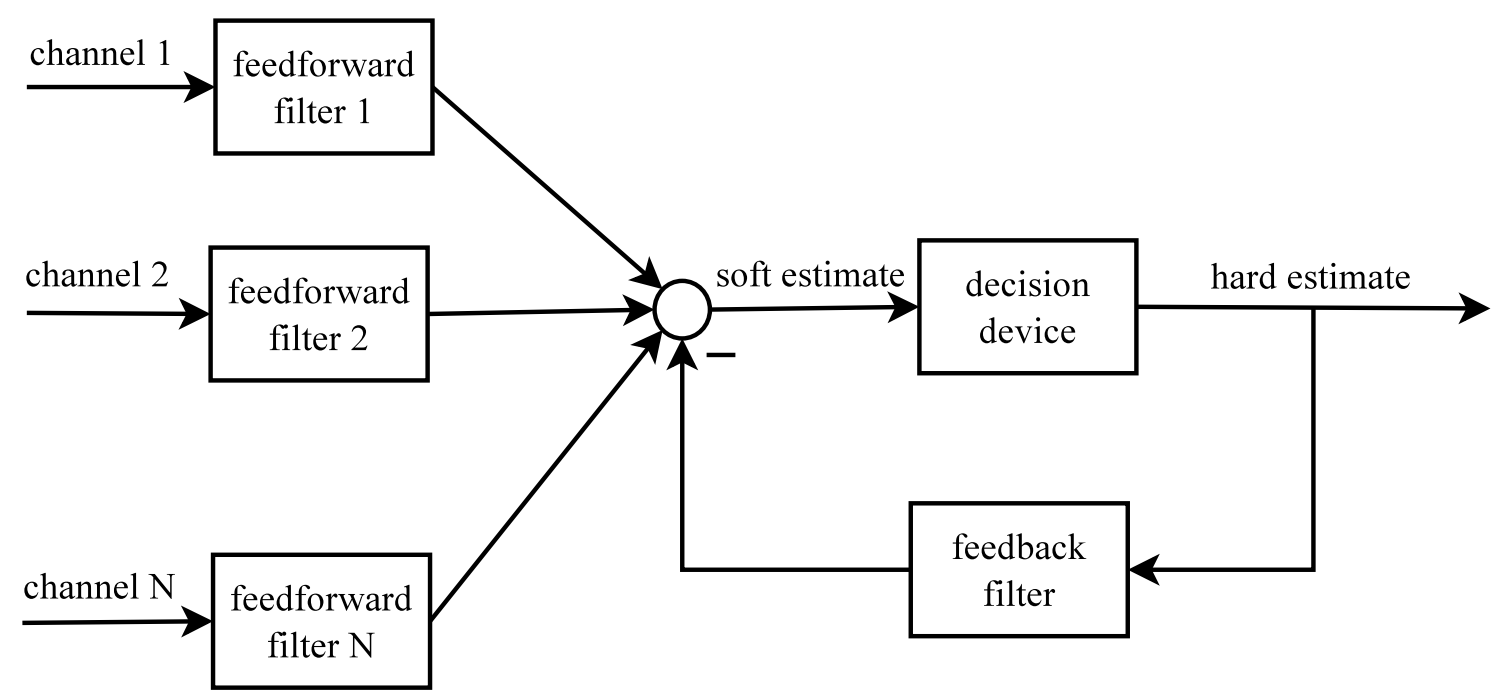

Figure 5-1: Block diagram of multi-channel decision feedback equalizer.

FB filter processes the equalizer's outputs (i.e., estimates of the transmitted symbols) with the goal to remove remaining ISI caused by the channel and the FF portion of the equalizer. A decision device produces a hard estimate of the transmitted symbol from a soft-decision estimate, obtained from the combined FF and FB filtering. All the constituent filters are assumed to be finite impulse response (FIR) filters.

\subsubsection{MC-DFE: Analytical Framework}

A mathematical framework of the MC-DFE is presented [39]. The received signal is assumed to originate from a single source. A channel between the source and the $i$-th sensor $(i=1,2, \ldots, N)$ is modeled with a linear filter and additive noise. In such a model, a symbol $u_{i}(n)$, received by a sensor $i$ at discrete time $n$ is

$$
u_{i}(n)=\mathbf{g}_{i}^{H}(n) \mathbf{x}(n)+v(n),
$$

where $\mathbf{g}_{i}(n)$ is a vector form of the $i$-th channel impulse response at time $n$. The transmitted symbols that give rise to $u_{i}(n)$ are compactly represented with a column vector $\mathbf{x}(n)$.

Without loss of generality, all the channels are assumed to have the same length $L_{c}$. In addition, we assume the channels have the same lengths of the causal and 
anti-causal parts, denoted respectively by $L_{c}^{c}$ and $L_{c}^{a}$. Therefore, the vector $\mathbf{x}(n)$ is formatted as

$$
\mathbf{x}(n)=\left[\begin{array}{lllll}
x\left(n+L_{c}^{a}\right) & \ldots & x(n) & \ldots & x\left(n-L_{c}^{c}+1\right)
\end{array}\right]^{T}
$$

Similarly, the FF filters are assumed to have the same length $L_{\mathrm{ff}}$. Also, their lengths of the causal and anti-causal parts, denoted respectively by $L_{\mathrm{ff}}^{c}$ and $L_{\mathrm{ff}}^{a}$, are equal.

The $i$-th FF filter output at time $n$ is driven by the received symbols $u_{i}(n+$ $\left.L_{\mathrm{ff}}^{a}\right), \ldots, u_{i}(n), u_{i}(n-1), \ldots, u_{i}\left(n-L_{\mathrm{ff}}^{c}+1\right)$. They are collected in a column vector $\tilde{\mathbf{u}}_{i}(n)$ which is expressed as

$$
\tilde{\mathbf{u}}_{i}(n)=\mathbf{G}_{i}(n) \underline{\mathbf{x}}(n)+\mathbf{v}_{i}(n),
$$

where $\mathbf{G}_{i}(n)$ is the $L_{\mathrm{ff}}$-by- $\left(L_{\mathrm{ff}}+L_{c}-1\right)$ channel matrix, obtained by appropriately shifting and stacking $\mathbf{g}_{i}^{T}\left(n+L_{\mathrm{ff}}^{a}\right), \ldots, \mathbf{g}_{i}^{T}\left(n-L_{\mathrm{ff}}^{c}+1\right)$ into its rows. The transmitted symbols $x\left(n+L_{\mathrm{ff}}^{a}+L_{c}^{a}\right), \ldots, x\left(n-L_{\mathrm{ff}}^{c}-L_{c}^{c}+2\right)$ impacting $\tilde{\mathbf{u}}_{i}(n)$ are collected into a column vector $\underline{\mathbf{x}}(n)$. Similarly, $\mathbf{v}_{i}(n)$ is a compact vector representation of the noise samples influencing $\tilde{\mathbf{u}}_{i}(n)$.

Stacking up the vectors $\tilde{\mathbf{u}}_{1}(n), \tilde{\mathbf{u}}_{2}(n), \ldots, \tilde{\mathbf{u}}_{N}(n)$ into a column vector, a signal vector $\underline{\tilde{\mathbf{u}}}(n)$, which represents a signal received at the equalizer's FF section at time $n$, is constructed and given by

$$
\underline{\tilde{\mathbf{u}}}(n)=\underline{\mathbf{G}}(n) \underline{\mathbf{x}}(n)+\underline{\mathbf{v}}(n),
$$

where the noise vector $\underline{\mathbf{v}}(n)$ and $\left(N L_{\mathrm{ff}}\right)$-by- $\left(L_{\mathrm{ff}}+N-1\right)$ multi-channel matrix $\underline{\mathbf{G}}(n)$ are constructed in a similar manner by stacking up the corresponding constituents vertically.

The input to the FB filter at time $n$ is a sequence of the equalizer's estimates $\hat{x}(n-1), \ldots, \hat{x}\left(n-L_{\mathrm{fb}}\right)$, where $L_{\mathrm{fb}}$ is the FB filter length. These estimates are collected in a column vector $\hat{\mathbf{x}}(n)$. Note that the index $n$ corresponds to an index of 
a symbol that is being estimated.

A soft decision estimate $\hat{x}_{\text {soft }}(n)$ is evaluated as

$$
\hat{x}_{\mathrm{soft}}(n)=\mathbf{w}^{H}(n) \mathbf{u}(n),
$$

where $\mathbf{w}(n)=\left[\begin{array}{llll}\mathbf{w}_{1}^{T}(n) & \ldots & \mathbf{w}_{N}^{T}(n) & -\mathbf{w}_{f b}^{T}(n)\end{array}\right]^{T}$ is the equalizer's weight vector (i.e., its impulse response) and $\mathbf{u}(n)=\left[\begin{array}{ll}\underline{\tilde{\mathbf{u}}}^{T}(n) & \hat{\mathbf{x}}^{T}(n)\end{array}\right]^{T}$ is the input to the equalizer at time $n$. A hard decision estimate $\hat{x}(n)$ is computed from $\hat{x}_{\text {soft }}(n)$ and the constellation diagram of the signaling employed in the communication scheme.

As a final remark, note that the overall number of equalizer coefficients is

$$
m=N L_{\mathrm{ff}}+L_{\mathrm{fb}}
$$

\subsubsection{MC-DFE: Optimization of Weights}

The equalizer weights $\mathbf{w}(n)$ are evaluated with respect to some optimization criterion. A minimization of the mean square error (MSE) between the transmitted symbol $x(n)$ and its soft decision estimate $\hat{x}_{\text {soft }}(n)$ is one of the most popular approaches, in which the weight vector $\mathbf{w}(n)$ is chosen such that the signal prediction mean square error

$$
\xi(n)=\mathbf{E}\left[\left|x(n)-\hat{x}_{\text {soft }}(n)\right|^{2}\right]
$$

is minimized. The solution to this optimization problem is referred to as the MMSE receiver given by,

$$
\mathbf{w}_{\text {MMSE }}(n)=\mathbf{R}^{-1}(n) \mathbf{r}(n)
$$

where $\mathbf{R}(n)$ and $\mathbf{r}(n)$ are the ensemble correlation matrix of the input signal and crosscorrelation vector between the input and desired output signal. They are evaluated as

$$
\begin{aligned}
\mathbf{R}(n) & =\mathbf{E}\left[\mathbf{u}(n) \mathbf{u}^{H}(n)\right] \\
\mathbf{r}(n) & =\mathbf{E}\left[\mathbf{u}(n) x^{*}(n)\right] .
\end{aligned}
$$


The signal prediction MSE of the MMSE filter $\mathbf{w}_{\text {MMSE }}$ is after substituting (5.8) into (5.7), given by

$$
\sigma_{\mathrm{MMSE}}^{2}(n)=\mathbf{E}\left[|x(n)|^{2}\right]-\mathbf{r}^{H}(n) \mathbf{R}(n)^{-1} \mathbf{r}(n)
$$

The ensemble statistics are rarely known and are replaced by time-average statistics. The cost function for an exponential weighting of the time-average statistics is $[27]$

$$
C(n)=\sum_{i=1}^{n} \lambda^{n-i}\left|\mathbf{w}^{H}(n) \mathbf{u}(i)-x(i)\right|^{2},
$$

where $\lambda \leq 1$ is a positive forgetting factor which accommodates the time-variability of the channel by reducing the impact of past data which is less relevant than current data for the current estimation problem.

A weight vector which minimizes this cost function is evaluated via

$$
\mathbf{w}(n)=\hat{\mathbf{R}}^{-1}(n) \hat{\mathbf{r}}(n),
$$

where $\hat{\mathbf{R}}(n)$ and $\hat{\mathbf{r}}(n)$ are the exponentially weighted sample correlation matrix (SCM) and input-desired output cross-correlation vector, given by

$$
\begin{aligned}
\hat{\mathbf{R}}(n) & =\sum_{i=1}^{n} \lambda^{n-i} \mathbf{u}(i) \mathbf{u}^{H}(i) \\
\hat{\mathbf{r}}(n) & =\sum_{i=1}^{n} \lambda^{n-i} \mathbf{u}(i) x^{*}(i)
\end{aligned}
$$

Note that these quantities are also introduced in the context of channel estimation in Chapter 4.

In the Recursive Least Squares (RLS) implementation of (5.13), the inverse of the SCM is calculated recursively and thus the computational requirements are reduced from order $N^{3}$ to order $N^{2}$ where the total number of equalizer parameters is given by $N[27]$.

The described method of updating equalizer coefficients is referred to as a direct 
adaptation approach and is of our interest in this chapter. In comparison, the equalizer weights can also be calculated based upon the estimates of the channel impulse response for each sensor and the ensemble statistics of the observation noise. This approach is labeled as a channel estimate based equalization and is studied in [39].

\subsection{Performance Analysis of Equalization of Time- Varying Channels}

The first part of this section presents the theoretical analysis of the signal prediciton MSE corresponding to the MC-DFE operating in time-varying channel. The obtained characterization is numerically validated via Monte-Carlo simulations in the second part. The theoretical analysis is fairly general and the results hold for any least squares based equalization with non-trivial filter lengths.

\subsubsection{Theoretical Analysis of Signal Prediction MSE}

The signal prediction MSE in the soft decision (i.e., the summed outputs of the FF and FB filters in Fig. 5-1) is adopted as the performance metric. To model the channel non-stationarity, we assume the channel is time invariant over a finite time interval and that the equalizer time averaging window for the purpose of calculating equalizer filter coefficients is limited to this time interval length. These observations are processed by the MC-DFE equalizer which operates in a training mode. This means that the input to the FB filter are the true transmitted symbols as are the symbols $x(i)$ used in the calculation of $\hat{\mathbf{r}}(n)$ in (5.15). The analysis of training mode operation allows the analysis of the impact of channel time-variability and thus limited observations intervals to be handled in a clearer manner and provides useful insights into performance trade-offs. Other contributions to the performance analysis of equalizers also assume operation in the training mode [39], [38].

Intuitively, when infinitely many observations are used to train the equalizer weights, the LS based MC-DFE with the rectangular window (i.e., a forgetting fac- 
tor $\lambda=1$ ) approaches the MMSE MC-DFE equalizer for a time-invariant channel. The impulse response vector $\mathbf{w}_{\text {MMSE }}$ of the MMSE filter and the corresponding signal prediction MSE (also called the minimum achievable error (MAE)) $\sigma_{\text {MMSE }}^{2}$, given by (5.11), are conditioned on the impulse response of the transmission channel. Recall that the prediction error at the MMSE filter output is white and uncorrelated with the input [27].

A crucial point in our analysis is the observation that the least squares based adaptation of the equalizer's coefficients $\mathbf{w}(n)$ can be framed as a channel identification problem, where an unknown channel is the MMSE filter $\mathbf{w}_{\text {MMSE }}$ which processes the input $\mathbf{u}(n)$ and whose output is corrupted with a white noise process $q(n)$ of the power $\sigma_{\mathrm{MMSE}}^{2}$, such that a sequence of transmitted symbols $x(n)$ is produced, i.e.,

$$
x(n)=\mathbf{w}_{\mathrm{MMSE}}^{H} \mathbf{u}(n)+q(n) .
$$

Intuitively, as the number of observation vectors $\mathbf{u}(n)$ grows, the equalizer weight vector $\mathbf{w}(n)$ approaches that of the MMSE equalizer. Consequently, we view the DFE equalizer as an adaptive processor which is "trying" to get as close as possible to the MMSE processor. Since the statistics of the received process are unknown and estimated using time-domain averaging, the DFE equalizer behaves as an adaptive processor which is estimating the MMSE processor based on the received signal and the desired output. This concept is depicted in Fig. 5-2.

Therefore, a signal prediction error $\xi(n)$ is using (5.5) and (5.16) expressed as

$$
\begin{aligned}
\xi(n) & =x(n)-\hat{x}_{\mathrm{soft}}(n) \\
& =\boldsymbol{\epsilon}^{H}(n-1) \mathbf{u}(n)+q(n),
\end{aligned}
$$

where

$$
\boldsymbol{\epsilon}(n)=\mathbf{w}_{\mathrm{MMSE}}-\mathbf{w}(n)
$$

measures how far the estimated equalizer weight vector $\mathbf{w}(n)$ is from the optimal (MMSE) equalizer $\mathbf{w}_{\text {MMSE }}$. We refer to it as an equalizer estimation error. 


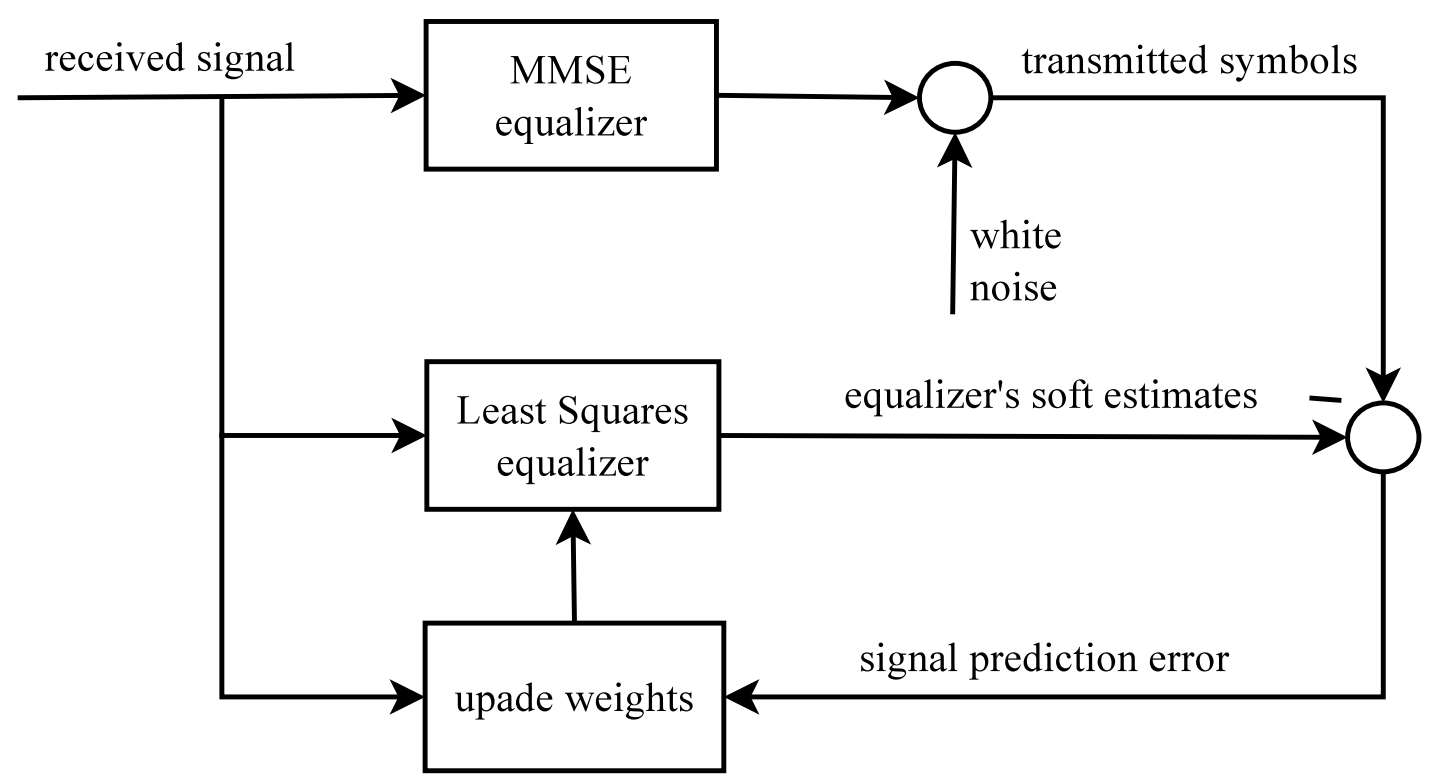

Figure 5-2: DFE performance analysis.

Since the noise process $q(n)$ is uncorrelated with the received signal $\mathbf{u}(n)$ and equalizer estimation error $\boldsymbol{\epsilon}(n-1)$, the signal prediction MSE is therefore given by

$$
\mathbf{E}\left[|\xi(n)|^{2}\right]=\mathbf{E}\left[\boldsymbol{\epsilon}^{H}(n-1) \mathbf{u}(n) \mathbf{u}^{H}(n) \boldsymbol{\epsilon}(n-1)\right]+\sigma_{\mathrm{MMSE}}^{2} .
$$

Using the facts that $\operatorname{tr}\{\mathbf{A B}\}=\operatorname{tr}\{\mathbf{B} \mathbf{A}\}$, for square matrices $\mathbf{A}$ and $\mathbf{B}$, and that the expectation and trace operators commute, the signal prediction MSE is further given by

$$
\mathbf{E}\left[|\xi(n)|^{2}\right]=\operatorname{tr}\left\{\mathbf{E}\left[\mathbf{u}(n) \mathbf{u}^{H}(n) \boldsymbol{\epsilon}(n-1) \boldsymbol{\epsilon}^{H}(n-1)\right]\right\}+\sigma_{\mathrm{MMSE}}^{2}
$$

Assuming that the received signal $\mathbf{u}(n)$ at time $n$ and the equalizer estimation error $\boldsymbol{\epsilon}(n-1)$ at time $n-1$ are uncorrelated, the signal prediction MSE is expressed as

$$
\mathbf{E}\left[|\xi(n)|^{2}\right]=\operatorname{tr}\left\{\mathbf{R E}\left[\boldsymbol{\epsilon}(n-1) \boldsymbol{\epsilon}^{H}(n-1)\right]\right\}+\sigma_{\mathrm{MMSE}}^{2}
$$

where $\mathbf{R}$ is the ensemble correlation matrix of the received process, defined in (5.9). Note that the first term in (5.21) is the signal prediction excess error. It appears because the number of symbols used to train the equalizer's coefficients is finite and the channel is time-varying. The analysis from here on assumes finite $n$ but that 
$\lambda=1$.

The equalizer estimation error $\boldsymbol{\epsilon}(n)$ can be expressed using (5.16) and (5.13) as

$$
\begin{aligned}
\boldsymbol{\epsilon}(n) & =\mathbf{w}_{\mathrm{MMSE}}-\hat{\mathbf{R}}^{-1}(n) \hat{\mathbf{r}}(n) \\
& =\mathbf{w}_{\mathrm{MMSE}}-\hat{\mathbf{R}}^{-1}(n) \sum_{i=1}^{n} \mathbf{u}(i)\left(\mathbf{u}^{H}(i) \mathbf{w}_{\mathrm{MMSE}}+q^{*}(i)\right) \\
& =-\hat{\mathbf{R}}^{-1}(n) \sum_{i=1}^{n} \mathbf{u}(i) q^{*}(i)
\end{aligned}
$$

A correlation matrix of the equalizer estimation error is using (5.22) evaluated as

$$
\begin{aligned}
\mathbf{E}\left[\boldsymbol{\epsilon}(n) \boldsymbol{\epsilon}^{H}(n)\right] & =\mathbf{E}\left[\hat{\mathbf{R}}^{-1}(n) \sum_{i=1}^{n} \mathbf{u}(i) q^{*}(i) \sum_{j=1}^{n} q(j) \mathbf{u}^{H}(j) \hat{\mathbf{R}}^{-1}(n)\right] \\
& =\sigma_{\mathrm{MMSE}}^{2} \mathbf{E}\left[\hat{\mathbf{R}}^{-1}(n)\right]
\end{aligned}
$$

where the last equality follows from the fact that noise process $q(i)$ is white and uncorrelated with the received signal $\mathbf{u}$.

Note from (5.22) that the correlation matrix of the estimation error of the equalizer weights scales with the variance of the MAE. That is, the more difficult the channel (not counting the dynamics), the more difficult it is to adapt and track the corresponding equalizer. Also, the more rapidly the channel fluctuates, the smaller $n$, and the larger the expected value of the inverse of the time-averaged correlation matrix.

To proceed further, we employ the results which characterize the expectation of the SCM inverse. If the received signal is Gaussian, which happens when the transmitted signal is Gaussian itself and/or the transmission channels $\mathbf{g}_{i}$ are long enough such that their outputs are approximately Gaussian by the central limit theorem, the SCM $\hat{\mathbf{R}}(k)$ is Wishart distributed and the expectation of its inverse is [27]

$$
\mathbf{E}\left[\hat{\mathbf{R}}^{-1}(n)\right]=\frac{\mathbf{R}^{-1}}{n-m-1}
$$

where $m=N L_{\mathrm{ff}}+L_{\mathrm{fb}}$ is the overall number of equalizer coefficients. 
For a general non-Gaussian case, the expectation of the SCM inverse is evaluated using the random matrix theory results. Namely, it is shown in Section 2.5.2 that if the order of the SCM $m$ and the number of observations $n$ grow large at the same rate such that $m / n \rightarrow c \in(0,1)$, the inverse of the rectangularly windowed SCM almost surely converges to the scaled inverse of the ensemble correlation matrix (2.54). Noting that the SCM $\hat{\mathbf{R}}$ as defined in (5.14) with $\lambda=1$ is a scaled version of the matrix model considered in Section 2.5.2, the convergence result (2.54) is rewritten as

$$
\left(\frac{1}{n} \hat{\mathbf{R}}(n)\right)^{-1} \rightarrow \frac{1}{1-c} \mathbf{R}^{-1} \quad \text { a.s. }
$$

As elaborated in Section 2.7, the expected inverse of the SCM is approximated with the limiting quantity in (5.25) such that

$$
\mathbf{E}\left[\hat{\mathbf{R}}^{-1}(n)\right] \approx \frac{\mathbf{R}^{-1}}{n-m}
$$

Finally, substituting (5.24) into (5.23) and the obtained result into (5.21) yields the exact expression for the signal prediction MSE when the received signal is Gaussian,

$$
\mathbf{E}\left[|\xi(n)|^{2}\right]=\frac{n-2}{n-\left(N L_{\mathrm{ff}}+L_{\mathrm{fb}}\right)-2} \sigma_{\mathrm{MMSE}}^{2}
$$

which is valid when $n>N L_{\mathrm{ff}}+L_{\mathrm{fb}}+2$. Note that this constraint comes from (5.24) and carries no practical insights.

Similarly, substituting (5.26) into (5.23) and the obtained result into (5.21) yields an approximation for the signal prediction MSE when the received signal has general statistics,

$$
\mathbf{E}\left[|\xi(n)|^{2}\right] \approx \frac{n-1}{n-\left(N L_{\mathrm{ff}}+L_{\mathrm{fb}}\right)-1} \sigma_{\mathrm{MMSE}}^{2}
$$

which is valid for $n>N L_{\mathrm{ff}}+L_{\mathrm{fb}}+1$. Note that (5.28) also approximates Gaussian case.

The derived characterization reveals that the signal prediction MSE at the LSbased MC-DFE output is proportional to the signal prediction MSE at the MMSE 
equalizer output (i.e., the minimal achievable error, $\sigma_{\text {MMSE }}^{2}$ ), and the proportionality constant does not depend on the channel impulse response. Note however that $\sigma_{\text {MMSE }}^{2}$ does depend on the noise and channel characteristics as well as the lengths of the equalizer's feedforward and feedback filters. A similar relationship between the SINR's at the outputs of the linear equalizer and its corresponding MMSE equalizer is obtained in [38].

\subsubsection{Numerical Validation}

The derived characterization for the signal prediction MSE is validated via simulations. In the first example we consider a single LTI channel whose impulse response is shown in Fig. 5-3. The transmitted symbols are binary $\{+1,-1\}$ with uniform distribution. They are transmitted through the considered channel and noise is added to the obtained symbols. The Gaussian distributed noise is non-white and generated such that its correlation properties correspond to the ambient ocean noise [12]. The received symbols are processed by a single channel DFE equalizer.

The comparison between the simulated and theoretically computed signal prediction MSE when the received SNR is $10 \mathrm{~dB}$ and the DFE equalizer has 22 taps in the FF filter and 20 taps in the FB filter is shown in Fig. 5-4. As can be observed, the derived characterization (5.28) accurately predicts the MSE performance.

In the second example, we consider the transmission of a binary sequence $\{+1,-1\}$ through a 5-channel LTI transmission system. The impulse response of one of the channels in shown in Fig. 5-3. The impulse responses of other channels are obtained by randomly perturbing the impulse response shown in Fig. 5-3. The additive noise is Gaussian distributed and generated such that its power density spectrum corresponds to the ambient ocean noise [12]. The received symbols are processed by a 5-channel DFE equalizer.

The comparison between the simulated and theoretically computed signal prediction MSE when the received SNR is $10 \mathrm{~dB}$ and the DFE equalizer has 10 taps in each sensor FF filter and 10 taps in the FB filter is shown in Fig. 5-5. As can be observed, the derived characterization (5.28) accurately predicts the MSE performance. 


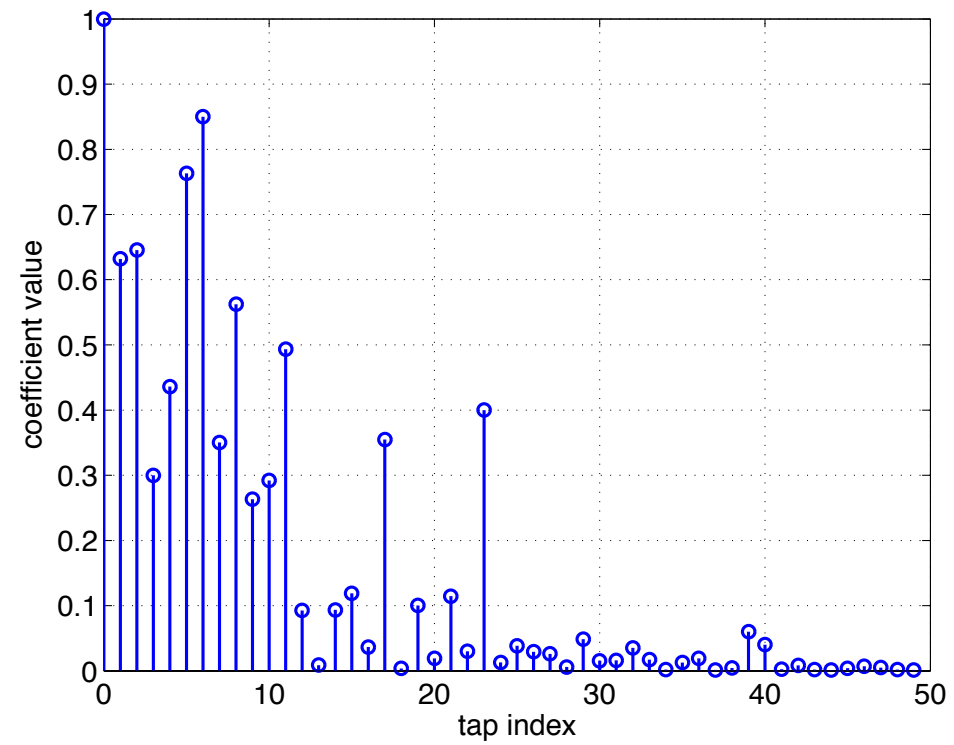

Figure 5-3: Channel impulse response used in simulations.

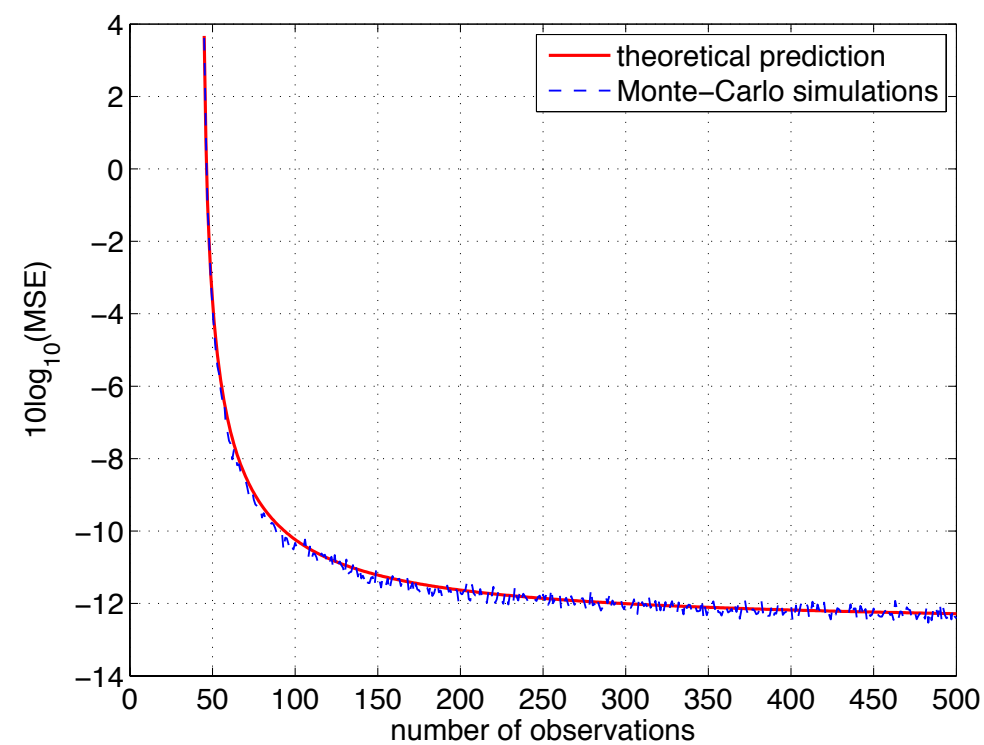

Figure 5-4: Signal prediction MSE versus number of observations for DFE equalizer with 22 taps in FF and 20 taps in FB filter. The transmission channel has length 50 and its impulse response is shown in Fig. 5-3. The noise is colored (ocean ambient noise) and the received SNR is $10 \mathrm{~dB}$. 


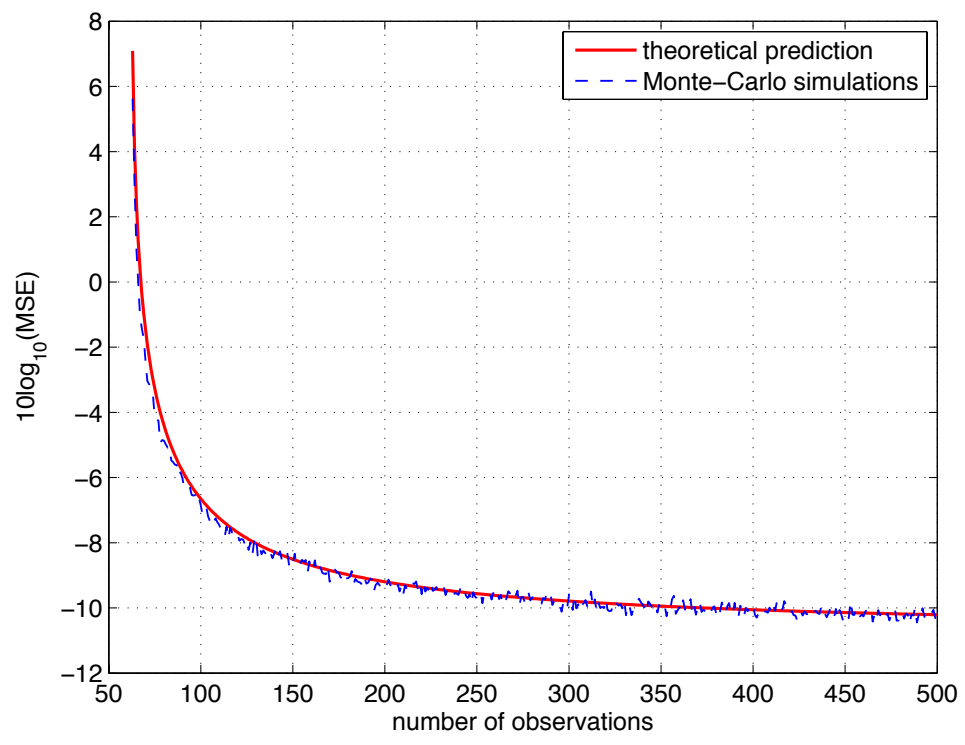

Figure 5-5: Signal prediction MSE versus number of observations for 5-channel DFE equalizer with 10 taps in each FF and 10 taps in FB filter. The noise is colored (ocean ambient noise) and the received SNR is $10 \mathrm{~dB}$.

\subsection{Equalizer Design: Optimal Number of Coeffi- cients}

This section considers a problem of determining an optimal number of coefficients in a multi-channel DFE equalizer which optimizes the signal prediction MSE. We assume the number of sensors (channels) of a multi-channel equalizer is fixed and analyze how the number of coefficients contained in the equalizer's constituent filters impacts the signal prediction performance.

\subsubsection{Insights into the Expression for Signal Prediction MSE}

The insights into effects that impact the optimal number of coefficients which minimizes the signal prediction MSE could be gained from the characterization of the signal prediction MSE (5.28).

Namely, the constant of proportionality in (5.28) is an increasing function of the overall number of equalizer weights $m$ when the number of observations $n$ is fixed. On 
the other hand, the MAE $\sigma_{\text {MMSE }}^{2}$ can be shown to be a monotonically non-increasing function of the equalizer length $m$. Therefore, the signal prediction MSE is minimized at a point where these two competing effects are balanced.

In other words, the optimal equalizer is a trade off between the MMSE requirements and those justified by the RMT insights. From the point of view of minimizing the MAE, the fact that the channel has a finite impulse response indicates that the best filter should be an IIR filter. However, the best estimate of the sample correlation matrix $\hat{\mathbf{R}}(n)$ when the number of stationary observations, $n$, is fixed (and controlled by the channel) is achieved if the order of the SCM, $m$, is 1 , i.e., if there is only one equalizer coefficient.

To illustrate how the number of stationary observations, $n$, impacts the quality of the SCM, we recall the Marcenko-Pastur law from Section 2.5.3. As shown in Fig. 2-1, the spread of the eigenvalues of the SCM corresponding to observations of zero mean, unit power white noise around the ensemble eigenvalue depends on the parameter $c$, whose inverse represents the number of observations per degree of freedom. Thus, as the value of parameter $c$ decreases, the eigenvalues of the SCM concentrate around the ensemble eigenvalue and hence, the SCM more accurately estimates the ensemble correlation matrix. The same reasoning applies when the input process is colored. That is, the ensemble correlation matrix is not the scaled identity matrix. Again, the eigenvalues of the SCM are spread around their ensemble counterparts and as the number of observations per dimension, $1 / c$, increases, the sample eigenvalues concentrate around ensemble eigenvalues.

Another insightful characterization of the signal prediction MSE is obtained by representing it as a sum of the MAE and excess error as in (5.20). Namely, (5.28) is equivalently expressed as

$$
\mathbf{E}\left[|\xi(n)|^{2}\right] \approx \sigma_{\mathrm{MMSE}}^{2}+\frac{m}{n-m-1} \sigma_{\mathrm{MMSE}}^{2}
$$

Note that the excess error is viewed as a product of the factor which depends only on the number of equalizer weights $m$ and averaging interval $n$ and the MAE $\sigma_{\text {MMSE}}^{2}$, 
which is a function of the channel impulse response and number of coefficients $m$.

The conclusion that the optimal number of equalizer coefficients is a trade off between the MMSE requirements and those justified by the RMT insights is numerically illustrated in the following part.

\subsubsection{Numerical Illustration}

In the first example, we consider an LTI channel whose impulse response is shown in Fig. 5-3. The received signal is processed through a single channel DFE equalizer. The signal prediction MSE corresponding to the MMSE equalizer coefficients is evaluated using (5.11). Its dependence on the number of taps contained in the FF and FB filters is shown in Fig. 5-6 for SNR of $0 \mathrm{~dB}$ (top figure) and SNR of $10 \mathrm{~dB}$ (bottom figure). As expected, the signal prediction performance improves as the number of equalizer coefficients increases.

A trade off between the MMSE requirement for longer filters and random matrix theory insight which favors shorter equalizers, is visualized by showing the dependence of the signal prediction MSE on the FF and FB filter lengths. This dependence is shown in Fig. 5-7 for the received SNR of $0 \mathrm{~dB}$ (top figure) and $10 \mathrm{~dB}$ (bottom figure). We assume the adaptation of equalizer weights is performed with 150 stationary symbols.

The number of coefficients in the constituent filters of the optimal DFE which minimizes the signal prediction MSE for different SNR's is shown in Table 5.1. The channel impulse response is as shown in Fig. 5-3 and the averaging interval is 150. As shown in table, for a given, fixed averaging interval, the optimal number of coefficients increases with SNR. In addition, the optimal distribution of coefficients between the constituent filters with increasing SNR is such that the number of coefficients in the FF filter decreases, while the number of coefficients in the FB filter increases.

Intuitively, the FF filter is the only one that can attenuate the noise. On the other hand, both filters attenuate the inter-symbol interference. However, the FF filter can only attenuate ISI to the extent that it has a different temporal signature than the desired symbol. Consequently, more coefficients are allocated to the FF filter at low 

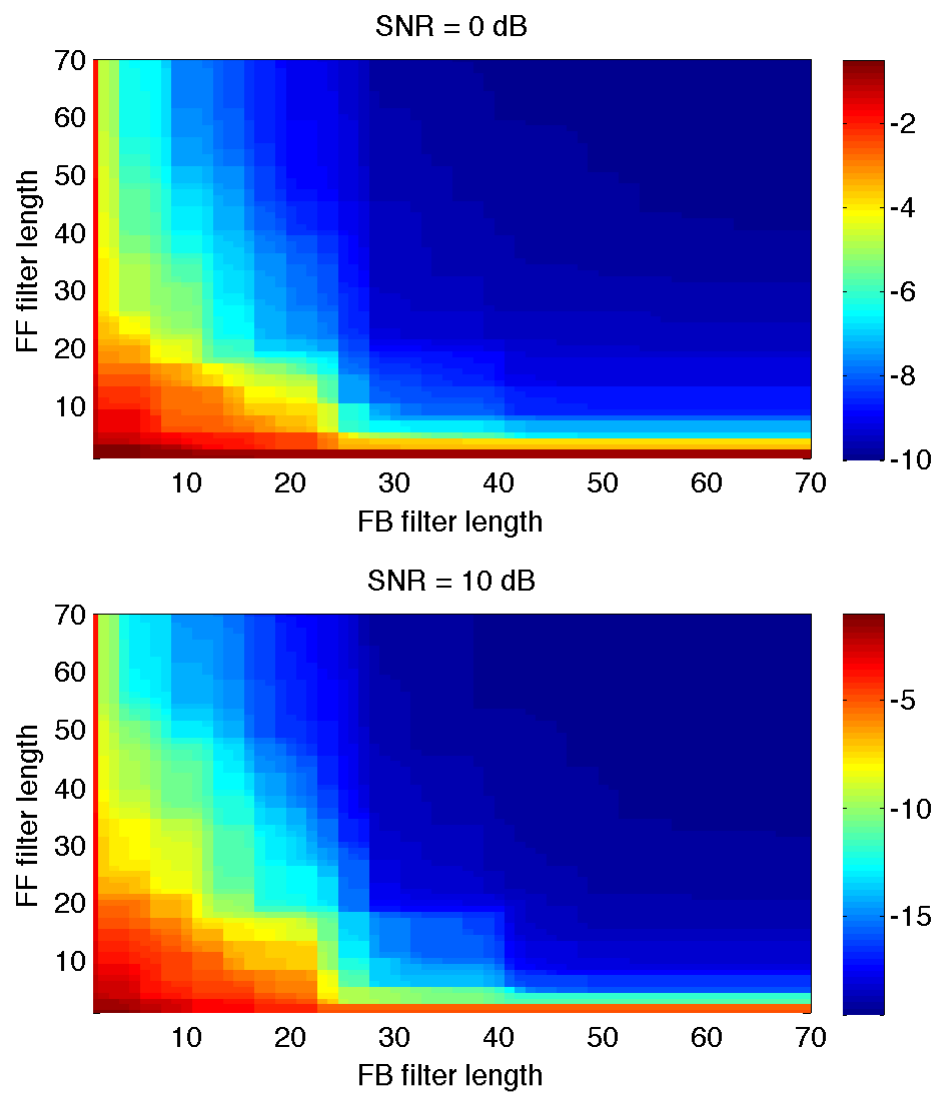

Figure 5-6: The minimum achievable signal prediction MSE in $\mathrm{dB}$ versus number of taps in FF and FB filter of the $\mathrm{DFE}$ equalizer for $\mathrm{SNR}=0 \mathrm{~dB}$ (top figure) and $\mathrm{SNR}=10 \mathrm{~dB}$ (bottom figure). The impulse response of the transmission channel is given in Fig. 5-3. 

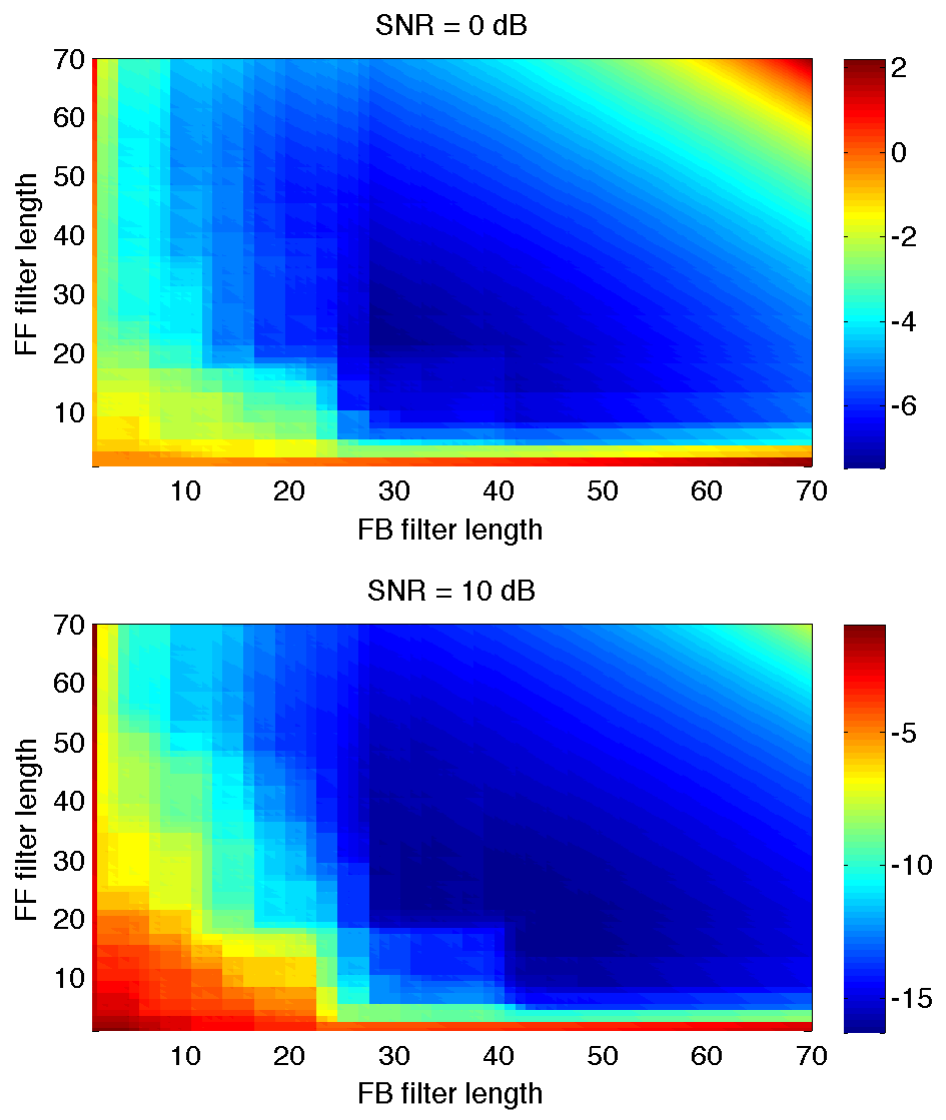

Figure 5-7: Analytically computed signal prediction MSE in dB versus number of taps in $\mathrm{FF}$ and $\mathrm{FB}$ filters of the $\mathrm{DFE}$ equalizer for $\mathrm{SNR}=0 \mathrm{~dB}$ (top figure) and $\mathrm{SNR}=10$ $\mathrm{dB}$ (bottom figure). The impulse response of the transmission channel is given in Fig. 5-3. The number of snapshots ( $\approx$ number of received symbols) is 150 . 
Table 5.1: Optimal Number of Coefficients

\begin{tabular}{|c||c|c|c|c|c|c|c|c|}
\hline SNR & $0 \mathrm{~dB}$ & $5 \mathrm{~dB}$ & $10 \mathrm{~dB}$ & $12 \mathrm{~dB}$ & $14 \mathrm{~dB}$ & $15 \mathrm{~dB}$ & $20 \mathrm{~dB}$ & $25 \mathrm{~dB}$ \\
\hline$L_{\mathrm{ff}}$ & 22 & 22 & 22 & 16 & 15 & 15 & 15 & 15 \\
\hline$L_{\mathrm{fb}}$ & 28 & 31 & 41 & 48 & 48 & 48 & 48 & 48 \\
\hline \hline$m$ & 50 & 53 & 63 & 64 & 63 & 63 & 63 & 63 \\
\hline
\end{tabular}

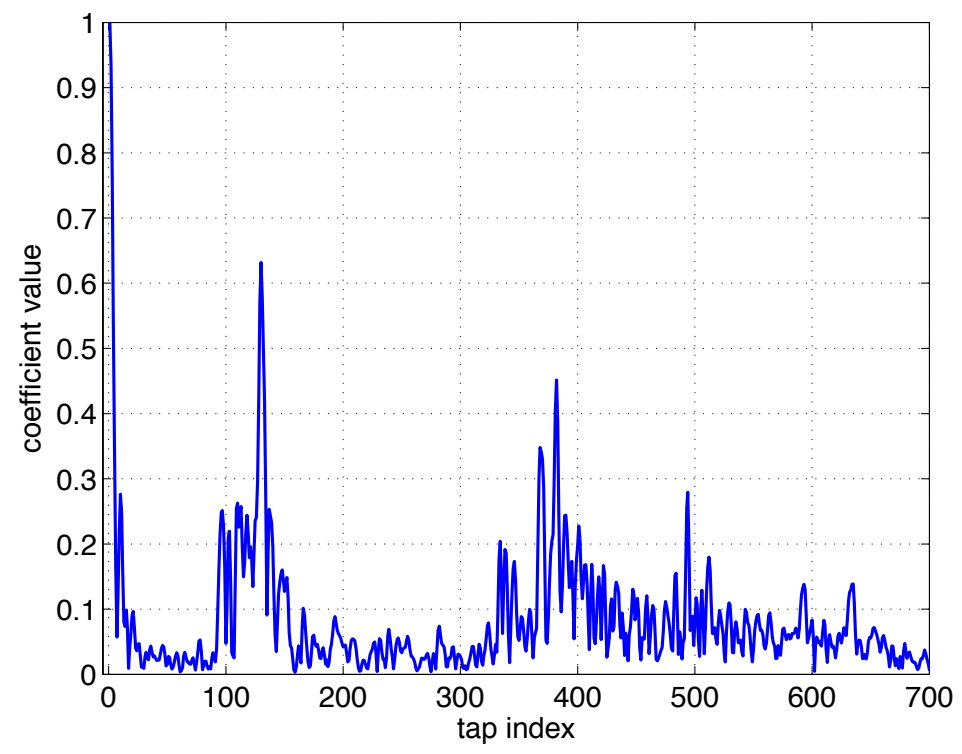

Figure 5-8: Channel impulse response at one of the sensors in KAM11 experiment.

SNR than at high SNR in order to reduce the noise. On the other hand, when SNR gets large, the ISI is the dominant source of distortion so that the optimal number of coefficients in the FB filter increases in order to eliminate the ISI.

In the second example we consider optimal MC-DFE design for the underwater acoustic communication channel observed during the KAM11 field experiment [28]. The number of channels (sensors) is 5 and a snapshot of a channel impulse response between a source and one of the sensors is shown in Fig. 5-8. The dependence of the signal prediction MSE on the lengths of FF and FB filters for received SNR of $22 \mathrm{~dB}$ and experimentally observed spatially correlated ambient noise is shown in Fig. 5-9. The number of stationary symbols used for adaptation is 500 . It can be noted that the optimal equalizer uses 16 taps in each FF filter and 10 taps in a FB filter and achieves signal prediction MSE of $-7.33 \mathrm{~dB}$. 


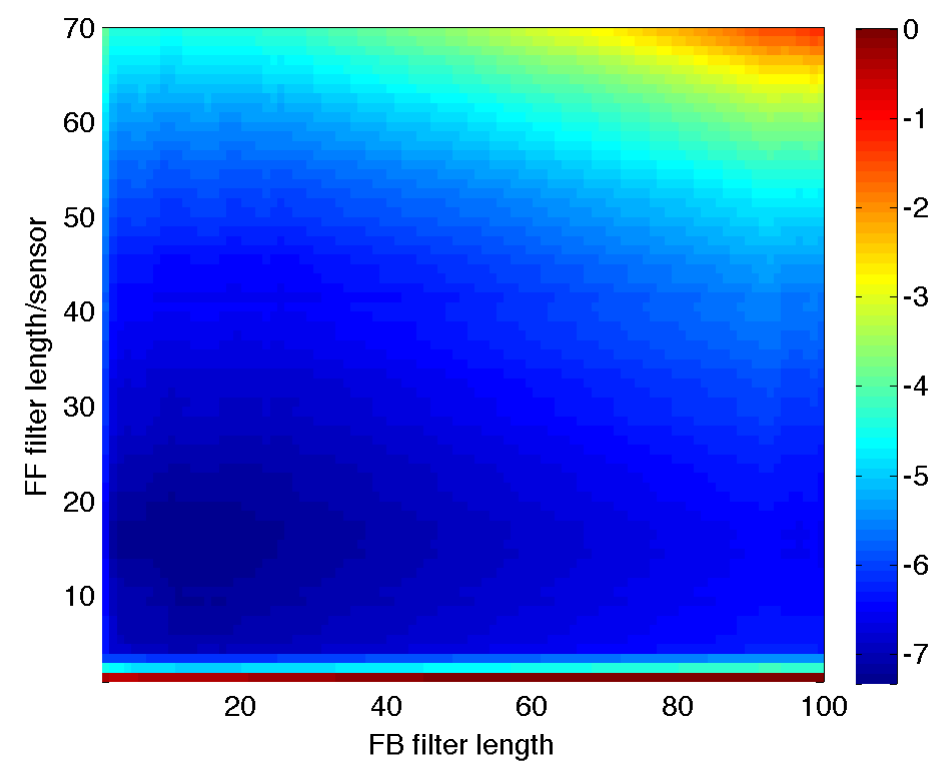

Figure 5-9: Signal prediction MSE in $\mathrm{dB}$ versus number of taps in FF filter per sensor and number of taps in FB filter. The number of sensors is 5, channel impulse response and spatially correlated ambient noise are as observed in field experiment. The received SNR is $22 \mathrm{~dB}$ and the channel coherence time is 500 symbols.

\subsection{Sparse Wideband Channel}

The previous section presents how to optimally choose the number of coefficients in an adaptive equalizer. It is concluded that the optimal number of coefficients is a trade off between two competing requirements. The presented insights are illustrated with the examples in which the number of sensors used in a multi-channel equalizer and the separation between them is fixed and predetermined.

This section develops a theoretical framework suitable for analyzing of and gaining insights in how the equalization performance depends on the array design, i.e., the selection of the number of and separation between the sensors. We first present the arrival model consisting of two wideband and spatially spread arrivals impinging on an array of sensors. The input correlation matrix and cross-correlation vector are then evaluated for the described arrival model.

Without loss of generality, this section considers linear multi-channel equalization. However, the model could be extended to include a multi-channel DFE. 


\subsubsection{Arrival Model}

We assume that a single source is isotropically transmitting a wideband signal of power 1 , confined within a frequency range between $\omega_{L}$ and $\omega_{U} \mathrm{rad} / \mathrm{s}$. The one-sided power spectrum density of the transmitted signal is given by ${ }^{1}$

$$
P_{x}(\omega)= \begin{cases}P_{0} & \text { if } \omega \in\left[\omega_{L}, \omega_{U}\right] \\ 0 & \text { otherwise. }\end{cases}
$$

We assume that on the receiver side, a linear vertical and uniformly spaced array of sensors with sensor separation $d$ is receiving the transmitted signal. The coordinate system has the origin at the position of the top-most sensor and its z-axis is along the array and oriented downwards. The projection of the spatial wavenumber vector $\mathbf{k}$ onto the $z$-axis is denoted with $k_{z}$. The directional cosine is defined as $u=\cos (\theta)$, where $\theta$ is the elevation angle with respect to the array such that $\theta=90^{\circ}$ corresponds to the broadside of the array.

To model the received signal, we assume that the underwater acoustic communication system is wideband as is the case for most single carrier systems. Also, the impulse response of the underwater acoustic communication channel is sparse. Furthermore, processing of data from a variety of underwater acoustic communication experiments shows that the acoustic energy is usually confined to a limited region of the delay-vertical wavenumber domain. As an example, the distribution of acoustic energy, received in the field experiment (KAM11) [28], across the elevation angle and delay domains, averaged over the time period of 60 seconds, is shown in Fig. 5-10.

Therefore, without loss of generality, we assume that the array receives acoustic energy from two different directions. The acoustic energy corresponding to each arrival is wideband and spatially spread in the angle domain.

To formalize the model, the transmission channel is viewed as a filter whose response in the $\omega-k_{z}$ domain has two non-zero, non-overlapping patches. Each patch

\footnotetext{
${ }^{1}$ The power amplifier is part of the communication channel in this model. Hence, the signal at the input of a power amplifier is the transmitted signal in our model.
} 


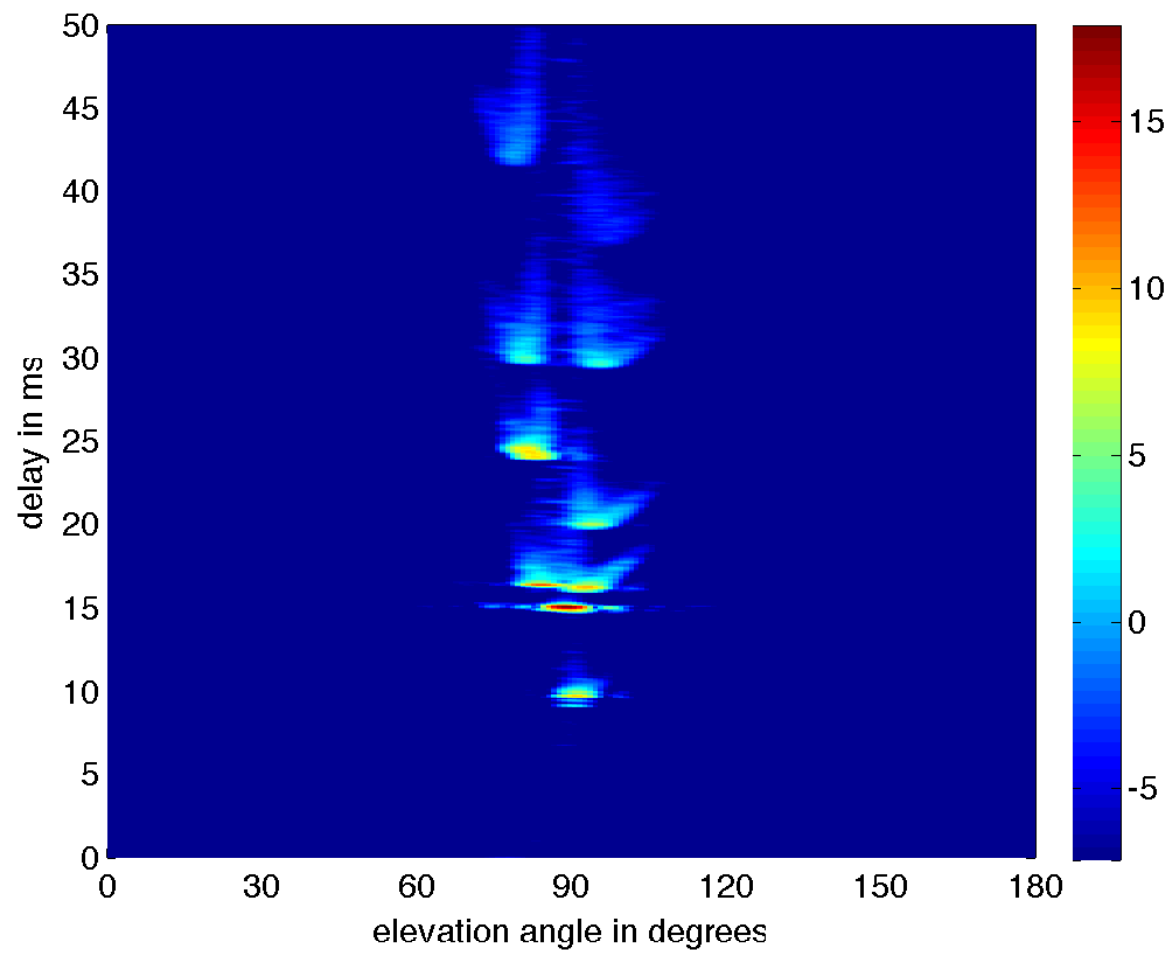

Figure 5-10: The distribution of acoustic energy received in the field experiment, averaged over time period of 60 seconds. 
gives rise to one arrival. We assume the arrivals come from non-overlapping ranges $\left[u_{n, l}, u_{n, u}\right], n=1,2$ in the directional cosine domain. The channel response is thus given $b^{2}$

$$
H\left(\omega, k_{z}\right)= \begin{cases}h_{1} & \omega \in\left[\omega_{l}, \omega_{u}\right] \text { and } k_{z}(\omega) \in\left[\frac{\omega}{c} u_{1, l}, \frac{\omega}{c} u_{1, u}\right] \\ e^{-j \omega \tau_{0}\left(k_{z}\right)} h_{2} & \omega \in\left[\omega_{l}, \omega_{u}\right] \text { and } k_{z}(\omega) \in\left[\frac{\omega}{c} u_{2, l}, \frac{\omega}{c} u_{2, u}\right] \\ 0 & \text { otherwise }\end{cases}
$$

where $h_{1}$ and $h_{2}$ are positive reals and $\tau_{0}\left(k_{z}\right)$ is the relative delay between the arrivals. Here we assume that the delay within each patch is much smaller than the relative delay between patches such that $\tau_{0}\left(k_{z}\right) \approx \tau_{0}$.

Given the channel model, the frequency-wavenumber spectrum of the received signal, $P_{y}\left(\omega, k_{z}\right)$, is then given by

$$
P_{y}\left(\omega, k_{z}\right)=\left|H\left(\omega, k_{z}\right)\right|^{2} P_{x}(\omega)
$$

The presented arrival model can be extended to mimic more realistic cases which include more arrivals, non-flat frequency responses over the possibly overlapping patches in the frequency-wavenumber domain, as well as a more general shape of an array of sensors. However, for the purpose of gaining insights in how sensor separation impacts the performance, the more important aspect of the model is that it accounts for wideband and spatially spread nature of the received signal.

In the following parts, we evaluate the correlation matrix and cross-correlation vector corresponding to the considered model.

\footnotetext{
${ }^{2}$ We assume that the power amplification is absorbed in the channel response.
} 


\subsubsection{Evaluation of Correlation Matrix $\mathbf{R}$}

The ensemble correlation matrix $\mathbf{R}$ is obtained from the space-time correlation function $\rho_{u u^{*}}(\tau, \Delta p)$ of the received signal, defined as

$$
\rho_{u u^{*}}(\tau, \Delta p)=\mathbf{E}\left[u(t, p) u^{*}(t-\tau, p-\Delta p)\right]
$$

where $u(t, p)$ is the continuous signal received at time $t$ and position $p$ along the $z$ axis. The sample of the continuous time signal $u(t, p)$ received at sensor $i$ at discrete time $n$ is denoted by $u_{i}(n)$. In general, the space-time correlation function describes the correlation between the signals received at points separated by vector $\Delta \mathbf{p}$ and at time instances separated by a delay $\tau$. However, we are only interested in correlation between signals received by sensors in an array. Since the sensors are aligned along the $z$-axis, the second argument in the space-time correlation function (5.33) is a scalar and describes the $z$-coordinate of a particular sensor.

The correlation function $\rho_{u u^{*}}(\tau, \Delta p)$ is evaluated from the frequency-wavenumber spectrum of the received signal and is given by [52]

$$
\begin{aligned}
\rho_{u u^{*}}(\tau, \Delta p) & =\frac{1}{(2 \pi)^{2}} \int_{\omega_{L}}^{\omega_{U}} \int_{-\omega / c}^{+\omega / c} P_{y}\left(\omega, k_{z}\right) e^{-j\left(\omega \tau-k_{z} \Delta p\right)} d \omega d k_{z} \\
& =\frac{1}{2 \pi} \int_{\omega_{L}}^{\omega_{U}} S_{y}(\omega, \Delta p) e^{-j \omega \tau} d \omega,
\end{aligned}
$$

where $S_{y}(\omega, \Delta p)$ is the temporal spectrum spatial correlation function. Note that since the $z$-axis is along the array and we are interested in correlations between signals received on an array, $\mathbf{k} \Delta \mathbf{p}=k_{z} \Delta p$.

The temporal spectrum spatial correlation function is for a channel response given in (5.31), evaluated in a closed form

$$
\begin{aligned}
S_{y}(\omega, \Delta p) & =\frac{1}{2 \pi} \int_{-\omega / c}^{+\omega / c} P_{y}\left(\omega, k_{z}\right) e^{j k_{z} \Delta p} d k_{z} \\
& =\frac{P_{0}}{\pi} \sum_{n=1}^{2}\left|h_{n}\right|^{2} \Delta k_{z, n} e^{-j \bar{k}_{z, n} \Delta p} \operatorname{sinc}\left(\Delta k_{z, n} \Delta p\right)
\end{aligned}
$$


where $\operatorname{sinc}(x)=\frac{\sin (x)}{x}$ and

$$
\bar{k}_{z, n}=\frac{\omega}{c} \frac{u_{n, l}+u_{n, u}}{2}, \Delta k_{z, n}=\frac{\omega}{c} \frac{\left|u_{n, u}-u_{n, l}\right|}{2} .
$$

are the wavenumbers corresponding to respectively the mean and width of the range of directional cosines pertaining to each arriving patch.

The space-time correlation function $\rho_{u u^{*}}(\tau, \Delta p)$ is then obtained by substituting (5.35) into (5.34) and performing integration. The final result can not be evaluated in a closed form for a given model and is computed numerically.

To specify the correlation matrix $\mathbf{R}$, we first note from the construction of the signal vector $\mathbf{u}$ and definition (5.9), that the correlation matrix $\mathbf{R}$ is a block matrix whose block in the position $(i, j)$ is

$$
\tilde{\mathbf{R}}_{i j}=\mathbf{E}\left[\mathbf{u}_{i} \mathbf{u}_{j}^{H}\right]
$$

where $i, j=1, \ldots, m$. Recalling that the observation vector $\tilde{\mathbf{u}}_{i}$ collects all the samples of the received signal at sensor $i$ which impact the output of the associated filter at a particular time instant, the element in the position $(t, s)$ of $\tilde{\mathbf{R}}_{i j}$ is given by

$$
\left[\tilde{\mathbf{R}}_{i j}\right]_{t, s}=\rho\left((t-s) T_{s},(i-j) d\right)
$$

where $d$ is the sensor separation (i.e., the sampling interval in spatial domain) and $T_{s}$ is the sampling interval in time domain.

\subsubsection{Evaluation of Cross-Correlation Vector $r$}

The cross-correlation vector $\mathbf{r}$ is obtained from the cross-correlation function $\rho_{u x^{*}}(\tau, p)$, defined as

$$
\rho_{u x^{*}}(\tau, p)=\mathbf{E}\left[u(t, p) x^{*}(t-\tau)\right]
$$

where $p$ is the position of the considered sensor along the $z$-axis.

Recall that the cross-correlation function between the input and output from a 
linear time invariant filter is given as the inverse Fourier transform of the crossspectral density. The cross-spectral density is evaluated as a product of the channel frequency response and power spectral density of the input process [26]. Analogously, the cross-correlation function $\rho_{u x^{*}}(\tau, p)$ is evaluated as

$$
\rho_{u x^{*}}(\tau, p)=\frac{1}{(2 \pi)^{2}} \int_{\omega_{L}}^{\omega_{U}} \int_{-\omega / c}^{\omega / c} P_{x}(\omega) H\left(\omega, k_{z}\right) e^{j\left(\omega \tau-k_{z} p\right)} d k d \omega .
$$

Since we are interested in evaluating the statistics of the signals received on the array, $p$ is a (scalar) distance between the origin and a particular sensor.

Denoting with $\tilde{S}_{u x^{*}}(\omega, p)$ the solution to the integration in (5.40) over $k_{z}$, the cross-correlation function is expressed as

$$
\rho_{u x^{*}}(\tau, p)=\frac{1}{2 \pi} \int_{\omega_{L}}^{\omega_{U}} \tilde{S}_{u x^{*}}(\omega, p) e^{-j \omega \tau} d \omega
$$

The function $\tilde{S}_{u x^{*}}(\omega, p)$ is for the considered model (5.31) evaluated in a closed form and given by

$$
\begin{aligned}
\tilde{S}_{u x^{*}}(\omega, p) & =\frac{1}{2 \pi} \int_{-\omega / c}^{+\omega / c} P_{x}\left(\omega, k_{z}\right) H\left(\omega, k_{z}\right) e^{-j k_{z} p} d k_{z} \\
& =\frac{P_{0}}{\pi} \sum_{n=1}^{2} h_{n} \Delta k_{z, n} e^{-j \bar{k}_{z, n} p} \operatorname{sinc}\left(\Delta k_{z, n} p\right)
\end{aligned}
$$

where $\Delta k_{z, n}$ and $\bar{k}_{z, n}$ are as defined in $(5.36)$.

The cross-correlation function $\rho_{u x^{*}}(\tau, p)$ is finally obtained by substituting $(5.42)$ into (5.41) and performing integration. The final result is for a given model computed numerically.

To specify the cross-correlation vector $\mathbf{r}$, we first note that it has a block structure whose $i$-th block $\tilde{\mathbf{r}}_{i}$ is the cross-correlation vector between the observation vector $\tilde{\mathbf{u}}_{i}$ and the transmitted signal $x$. Since $\tilde{\mathbf{u}}_{i}$ collects the time samples of the signal received at sensor $i$ which impact the output of the associated filter at a particular time instant, 
the $s$-th element in $\tilde{\mathbf{r}}_{i}$ is thus given by

$$
\left[\tilde{\mathbf{r}}_{i}\right]_{s}=\rho_{u x^{*}}\left(s T_{s}, i d\right)
$$

where $d$ and $T_{s}$ are the sampling intervals in, respectively, spacial and delay domains.

\subsection{Equalizer Design: Optimal Array Selection}

This section illustrates how the number of sensors and their separation impact the signal prediction performance of the MMSE and LS adapted multi-channel equalizer for a particular arrival structure. The last part presents experimental results which validate that the equalization performance is optimized for a finite sensor separation which is not necessarily equal to one half the shortest wavelength.

The dependence of signal prediction MSE on the number of sensors and their separation is examined by using (5.11) and (5.28) for a particular arrival structure. The theoretical framework presented in the previous section is used to evaluate the correlation matrix $\mathbf{R}$ and cross-correlation vector $\mathbf{r}$ corresponding to the considered arrival structure.

\subsubsection{Optimal Sensor Separation}

One of the challenges related to optimally configuring the multi-channel equalizer is the selection of separation between sensors. While the sensors in a MIMO system whose channel is characterized by rich scattering need to be sufficiently apart so that the signals at their outputs are uncorrelated [20], conventional wisdom is that array processing applications require that sensors be separated by no more than one half the shortest wavelength of the received signals [52]. However, because an inherently wideband signal is transmitted through a sparse underwater acoustic communication channel, selection of optimal sensor separation is a more subtle problem.

As an illustration, we consider a particular arrival model whose bandwidth is between $9 \mathrm{kHz}$ and $17 \mathrm{kHz}$, motivated by the KAM11 experiment [28]. For a sound 
speed of $1535 \mathrm{~m} / \mathrm{s}$, as observed in some of the KAM11 data epochs, the corresponding wavelengths are between $9.03 \mathrm{~cm}$ and $17.06 \mathrm{~cm}$.

Here we consider an arrival model for which the acoustic energy arrives from the ranges of elevation angles of $\left[89^{\circ}, 91^{\circ}\right]$ and $\left[84^{\circ}, 86^{\circ}\right]$. The corresponding channel responses are equal, i.e., $h_{1}=h_{2}$, and the relative delay between the arrivals is much larger than the delay within each patch and is equal to two sampling periods, i.e., $\tau_{0}=2 / F_{s}$, where $F_{s}=40 \mathrm{kHz}$ is the sampling frequency. Note that for a given signal bandwidth of $8 \mathrm{kHz}$, the arrivals are not resolvable in the delay domain. The ambient noise is directional and has power 1. It is confined within the range of elevation angles between $80^{\circ}$ and $100^{\circ}$. The signal-to-noise ratio (SNR) is $10 \mathrm{~dB}$. The linear multi-channel equalizer contains 10 sensors.

The dependence of the signal prediction MSE on sensor separation when the statistics of the input process are known such that the equalizer coefficients are evaluated using (5.8) and the equalizer has 1 tap in each sensor filter is shown in Fig. 5-11. The case when each sensor filter contains 5 taps is shown in Fig. 5-12. As can be observed, the performance is optimized for a finite sensor separation which is greater than one half the shortest wavelength of the signals of interest.

When the statistics of the input process are unknown and estimated from the received data, the signal prediction MSE is proportional to the signal prediction MSE corresponding to the MMSE processor, as given by (5.28). Hence, the dependence of the signal prediction MSE on sensor separation for the considered arrival model and multi-channel equalizer is as shown in Figures 5-11 and 5-12 up to an appropriate scaling of the vertical axes. The scaling depends on the averaging window size and overall number of coefficients.

Finally, as an illustration, the dependence of signal prediction MSE on the number of sensors and their separation when the equalizer weights are computed using the least squares solution (5.13) and the channel is non-stationary and approximately time-invariant over 500 symbols is shown in Fig 5-13. The signal prediction MSE is evaluated using (5.28). Under the constraint that each sensor filter has 5 taps, the signal prediction performance is optimized when equalizer contains 12 sensors and 


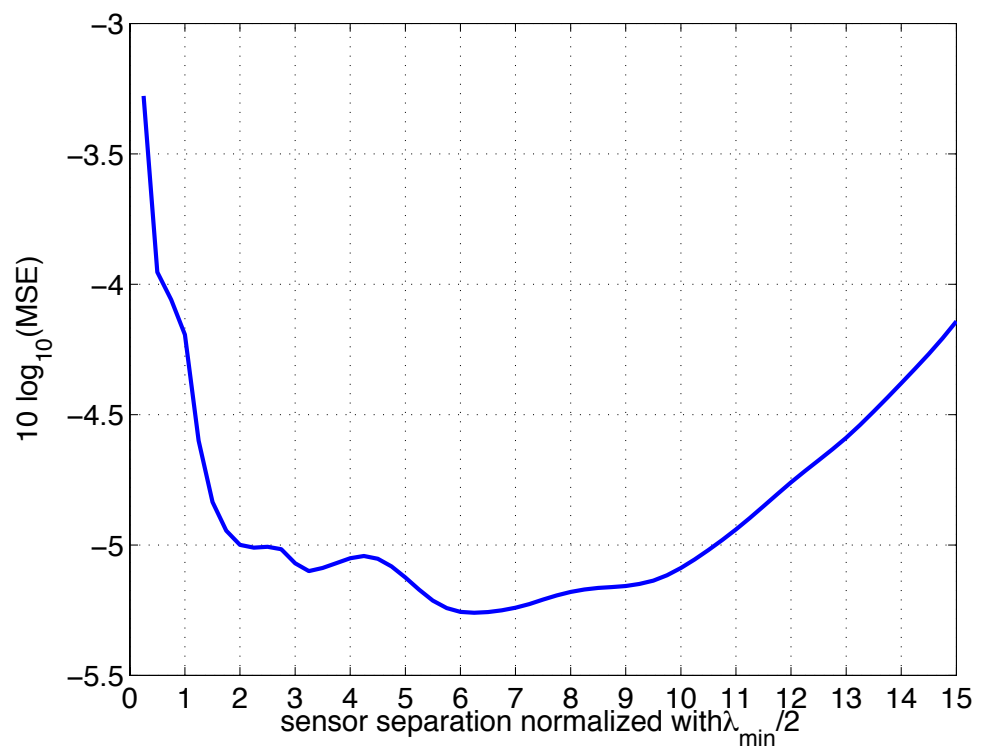

Figure 5-11: Signal prediction MSE versus sensor separation for MMSE equalizer. The sensor separation in normalized with the half-a-wavelength corresponding to the highest frequency of interest. Each of the 10 single sensor feedfoward filters has a length of 1 tap.

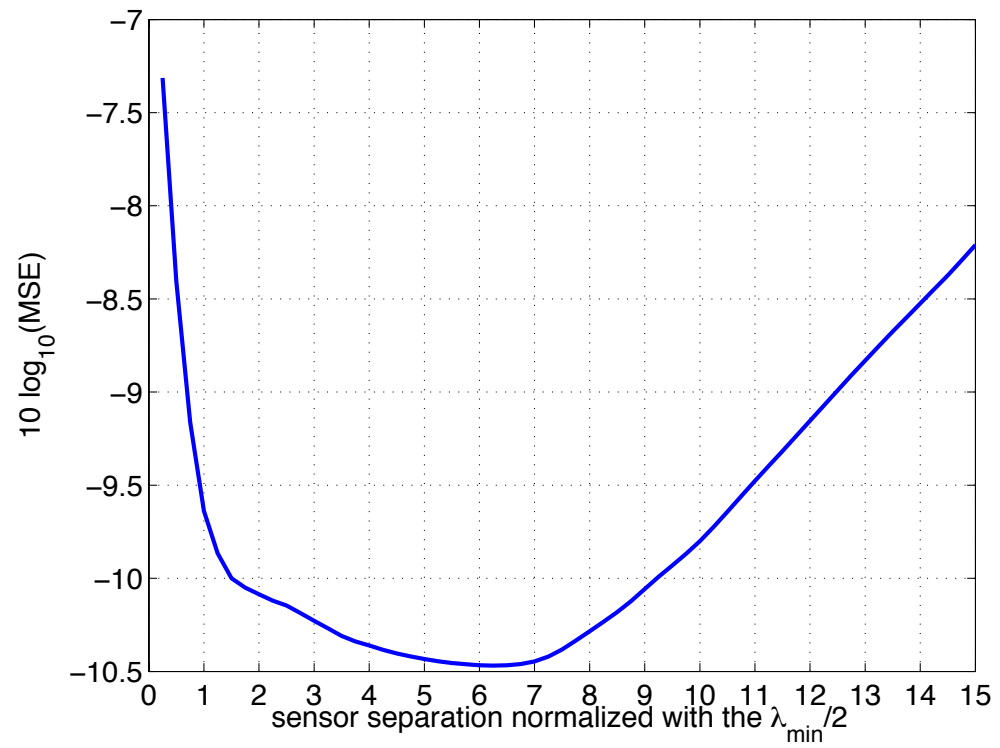

Figure 5-12: Signal prediction MSE versus sensor separation for MMSE equalizer. The sensor separation in normalized with the half-a-wavelength corresponding to the highest frequency of interest. Each of the 10 single sensor feedfoward filters has a length of 5 taps. 


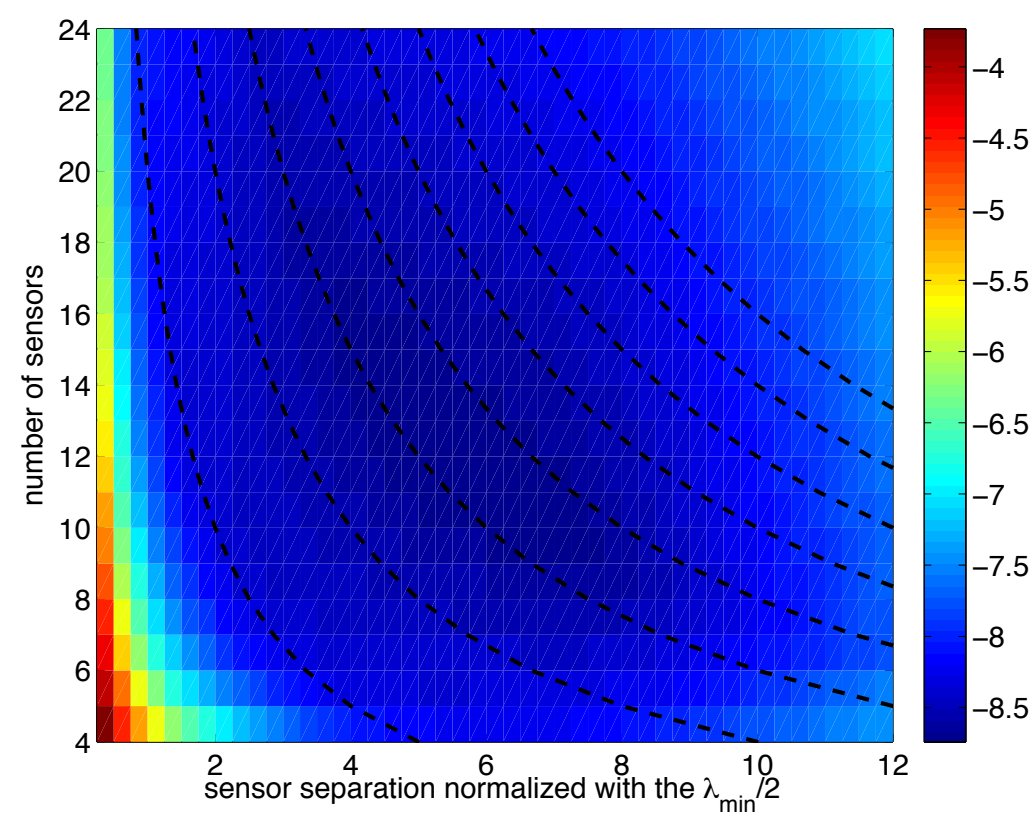

Figure 5-13: Signal prediction MSE versus number of sensors and sensor separation for LS equalizer. The sensor separation in normalized with the half-a-wavelength corresponding to the highest frequency of interest. The dashed lines are curves of constant aperture.

their separation is almost $6 \lambda_{\min }$, where $\lambda_{\min }=9.03 \mathrm{~cm}$. Note that the Note the lines of constant aperture shown in Fig. 5-13. This effect is examined in the following section.

\subsubsection{Optimal Array Aperture}

The dependence of optimal sensor separation on the number of sensors and number of taps per sensor filter is illustrated here using the same arrival model as considered in the previous section. The only distinction is that the relative delay between two arrivals is $\tau_{0}=5 / F_{s}$ so that longer sensor filters are needed in order to suppress the interference in the delay domain. Note that the arrivals are not resolvable in the delay domain.

The dependence of optimal sensor separation, normalized with half the shortest wavelength of the received signal, on the number of sensors when each sensor filter contains $L=7$ taps is shown in Fig. 5-14. The statistics of the input signal is assumed 


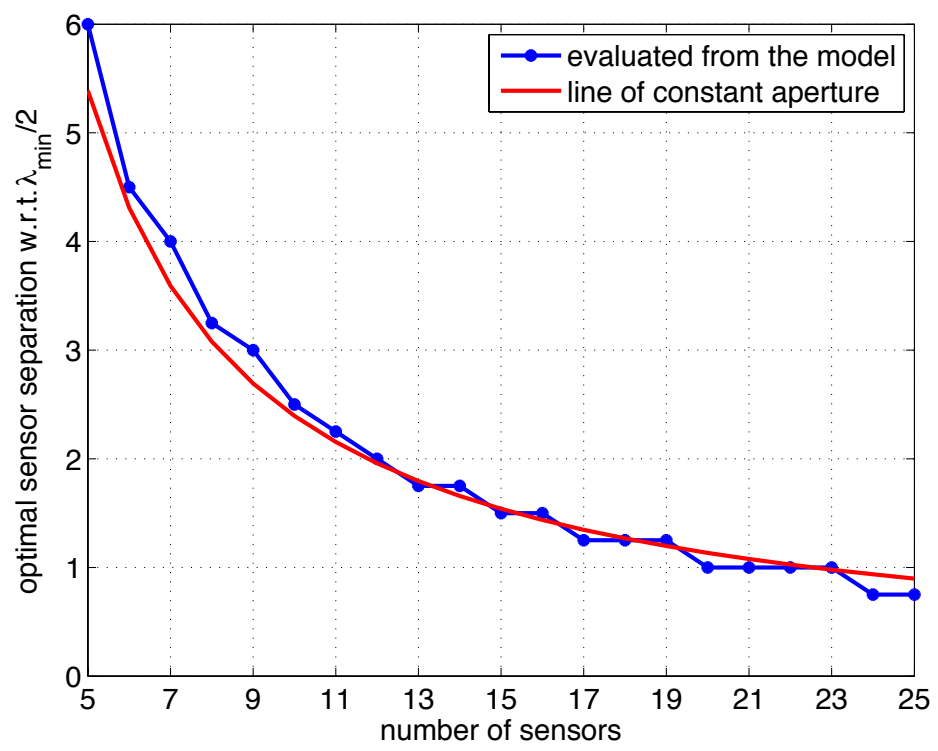

Figure 5-14: Optimal sensor separation versus number of sensors. Each sensor feedforward filter has 7 taps. Each point on the blue curve optimizes the signal prediction MSE in the theoretical model. The red curve is the line of constant aperture, obtained from averaging the apertures corresponding to each point on the blue curve.

known and the optimal sensor separation optimizes (5.11). The input correlation matrix $\mathbf{R}$ and cross-correlation vector $\mathbf{r}$ for the considered model are evaluated as described in Section 5.5.

As shown in Fig. 5-14, the optimal sensor separation decreases as the number of sensors increases in such a way that the array aperture is approximately constant. This is confirmed by plotting the constant aperture curve in Fig. 5-14, where the constant aperture is obtained from averaging the optimal apertures evaluated for the considered numbers of sensors.

The dependence of optimal sensor separation on the number of sensors $N$ and number of taps per sensor filter $L$ in the MMSE processor is shown in Fig. 5-15. As can be observed the optimal array aperture is approximately constant and independent of $L$ when $L \geq 6$. Recall that the relative delay between the arrivals in the considered example is $5 / F_{s}$ and the taps in a sensor filter are separated by $1 / F_{s}$. Therefore, the sensor filters containing $L \geq 6$ taps extend over both arrivals in the delay domain and the processor reasonably well suppresses the interference. 


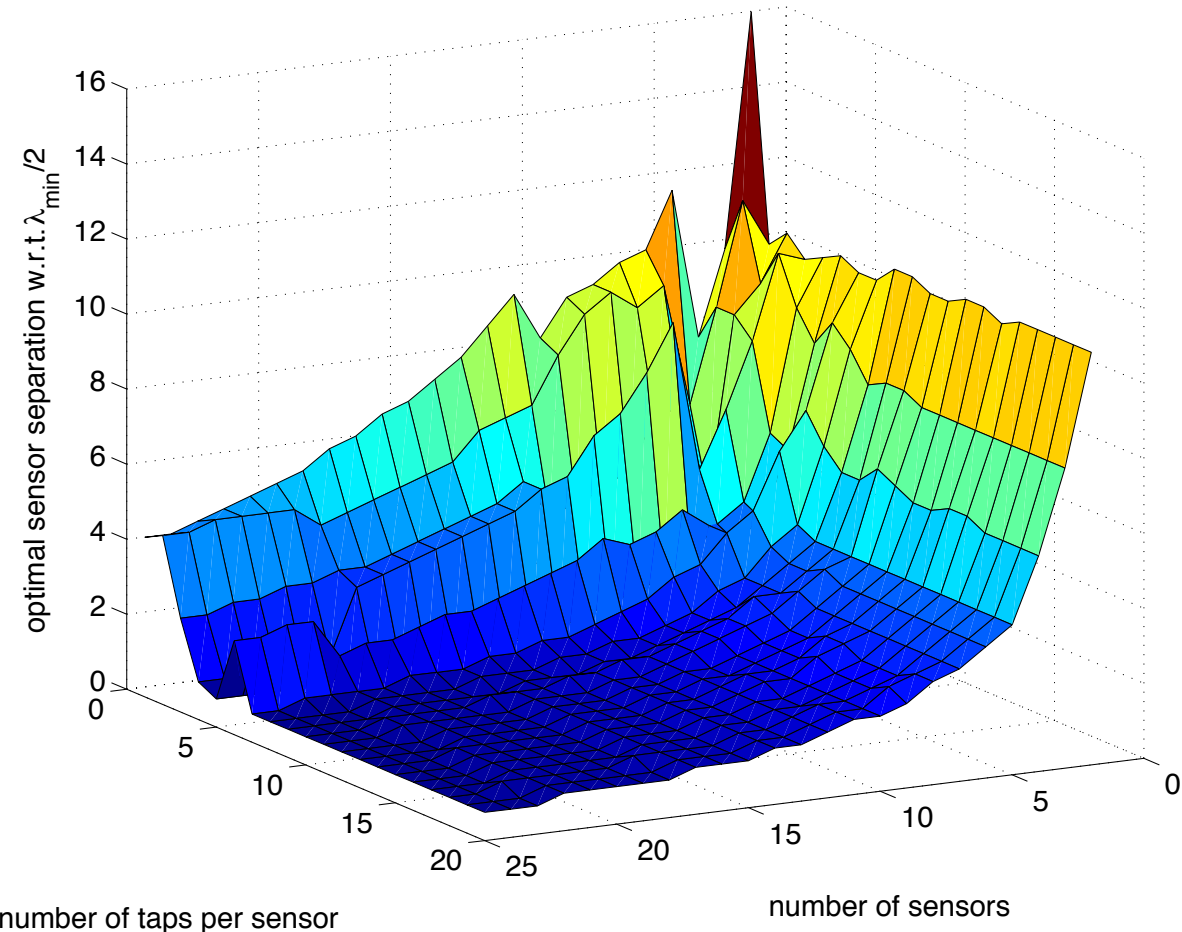

Figure 5-15: Optimal sensor separation as a function of the number of sensors and number of taps per sensor filter.

On the other hand, when $L \leq 5$, the optimal sensor separation tends to increase as the length of sensor filters $L$ decreases. In other words, given that the suppression capability is fairly limited in the delay domain due to short sensor filters, the aperture of the optimal processor increases so that the interference is better suppressed in the spatial domain. In addition, when the number of sensors $N$ is not unreasonably small (i.e., $N$ is above 4 in this example), the optimal aperture remains approximately constant and depends on $L$.

The behavior of optimal aperture remains the same when equalizer coefficients are evaluated using the LS algorithm. Namely, recall that the signal prediction MSE of the LS based equalizer is proportional to the signal prediction MSE corresponding to the MMSE processor. The constant of proportionality is a function of the number of coefficients and observation window length. Therefore, the optimal sensor separation which minimizes the signal prediction MSE for a given number of sensors $N$ and number of taps per sensor filter $L$ is the same for the MMSE and LS based processors. 
Finally, we conclude that the optimal aperture is determined by the arrival structure of the received signal. As long as the arrival structure remains unchanged during the observation period, the length of the observation interval does not impact the aperture of the optimal multi-channel processor.

\subsubsection{Experimental Evidence}

For experimental evidence that sensor separation is an important factor in determining equalizer performance, the following results obtained from processing the data collected in KAM11 field experiment [28] are useful. The underwater acoustic communication signal received at a vertical linear and uniformly spaced array is processed through a linear multi-channel equalizer and the transmitted symbols are detected. The equalizer contains 4 sensors and each sensor filter has 20 taps. The equalizer coefficients are adapted using the recursive least squares (RLS) algorithm using the forgetting factor $\lambda$ which yields the best bit error rate performance. The measured signal prediction MSE and BER performance versus sensor separation is shown in Fig. 5-16 in respectively top and bottom part. Note that the best signal prediction MSE and BER performance is achieved for $d=30 \mathrm{~cm}$. The minimal and maximal wavelengths corresponding to the signal of interest in the KAM11 experiment are respectively $9.03 \mathrm{~cm}$ and $17.06 \mathrm{~cm}$. Note that the optimal separation is approximately $6 \lambda_{\min } / 2$, which corresponds to the optimal sensor separation obtained for the arrival structure considered in the previous part (refer to Fig. 5-12). As a final remark, the linear equalizer is implemented in time domain and no Doppler compensation is employed.

\subsection{Conclusions}

The performance analysis and optimal design of multi-channel equalizers of timevarying channels is presented in this chapter. An analytical characterization of the signal prediction MSE achieved at the output of the multi-channel DFE equalizer operating in a non-stationary channel is first presented. The channel is modeled as 

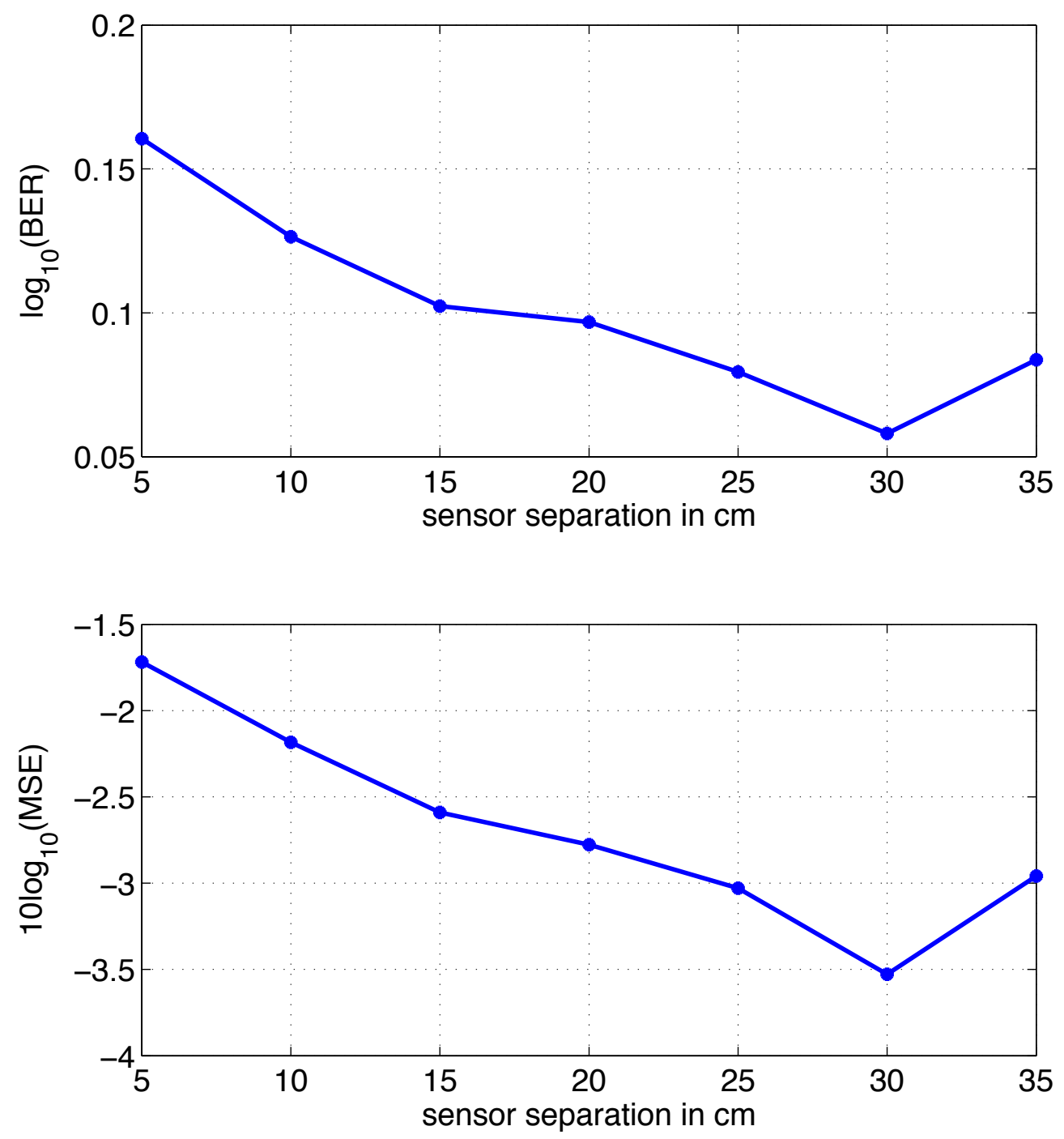

Figure 5-16: Experimentally measured signal prediction MSE (top figure) and BER (bottom figure) versus sensor separation for a 4-sensor linear equalizer with 20 taps per sensor filter. 
a frequency selective filter which is time invariant over short time intervals such that the number of stationary observations used to adapt the equalizer weights is finite and limited. The equalization problem is framed as a channel identification problem where an "unknown" channel is the MMSE equalizer. It is shown that the signal prediction MSE at the output of the LS-based MC-DFE equalizer is proportional to the signal prediction MSE at the output of the corresponding MMSE equalizer and the proportionality constant does not depend on the channel impulse response. The derived expression is validated via Monte-Carlo simulations.

Further, the chapter studies the problem of optimal equalizer design. Namely, the obtained characterization of the equalization performance highlights that the optimal number of equalizer coefficients is a trade off between the MMSE requirement for longer filters and the insight that finite number of observations can effectively adapt only a limited number of equalizer weights. This is validated via Monte-Carlo simulations and processing of experimentally collected data.

Finally, an analysis of how performance of multi-channel equalizer of time-varying underwater acoustic communication channels depends on the number of sensors and the separation between them is presented. While the sensors in a conventional MIMO system need to be sufficiently apart so that the signals at their outputs are uncorrelated, conventional wisdom is that array processing applications require that sensors be separated by no more than one half the shortest wavelength of the received signals. However, the selection of optimal sensor separation is a more subtle problem in the context of underwater acoustic communications. To study this problem, we introduce an arrival model which accounts for wideband and spatially spread nature of the received underwater acoustic communication signals. Using a particular arrival structure we show that the performance is optimized for a non-trivial selection of the number of sensors and their separation. Finally, these findings are confirmed by processing the experimental data.

As a possible future research, the presented arrival model could be extended such that the dependence of optimal number of sensors on total wavenumber spread and wavenumber spread of the arrivals we aim to separate, is revealed. Also, the ar- 
rival model could be generalized to mimic more realistic arrival structures so as to include more arrivals, non-flat frequency response over the patches in the frequencywavenumber domain and to account for non-negligible delay within the arrival. A possible application of the developed results and insights might be in the design of subarray-based equalization method for rapidly varying communication channels.

Although the emphasis in this chapter is on underwater acoustic communications, the derived characterizations and gained insights are applicable in other settings. As such, the derived characterization of equalization performance holds in other applications that rely on least squares based adaptation in the deficient sample support regime using non-trivial constituent filter lengths.

Another potential application area is ultra-wide band communications and, in particular, an increasingly popular $60 \mathrm{GHz}$ communications. Namely, since an underwater acoustic communication channel is wideband, it shares some features with an ultra-wideband radio communication channel. Specifically, both channels are sparse and the received signals consist of a limited number of wideband and spatially spread arrivals. In addition, given the fact that the receivers in ultra-wideband communication systems are envisioned to contain many antennas (i.e., massive MIMO), the optimal receiver design may benefit from our analysis and the insights in how sensor separation impacts the equalization performance. 


\section{Chapter 6}

\section{Conclusions and Future Work}

The coefficients in adaptive processors are often obtained as a solution to an optimization problem whose objective function is based on the second order statistics of the input process. Second order statistics are usually used because they arise naturally in squared error criteria involving linear signal and processing models and the estimation of higher order statistics is often difficult due to limited data with which to estimate statistics. In addition, adaptive processing with higher order statistics requires more computations, which is usually a scarce resource.

The adaptive processor weights, obtained as a solution to an optimization problem with objective function based on second order statistics, depends on the ensemble correlation matrix of the input process. However, the ensemble correlation matrix is often unknown and is estimated from the observed data. The sample correlation matrix (SCM) is a widely used estimator or building block in other estimators of the ensemble correlation matrix. The SCM accurately estimates the ensemble correlation matrix when the number of observations is sufficiently many times larger than the number of coefficients, which is rarely the case in practice.

The SCM evaluated from deficient sample support might significantly differ from the ensemble correlation matrix. The problem of deficient sample support arises as a result of one or more of the following reasons. First, the statistics of the input signal might be non-stationary because the signal has propagated through a time-varying environment, such as in terrestrial wireless communications. Second, the length of 
the observation interval that can be used to estimate the time-varying statistics might not be sufficient, such as in medical applications. Finally, the number of dimensions might be very large so that the number of observations is small compared to the number of dimensions, such as in modern radar and sonar systems.

This thesis studied the problems associated with adaptive processing based on second order statistics in the deficient sample support regime. The applications of adaptive processing considered in the thesis were adaptive beamforming for spatial spectrum estimation, tracking of time-varying channels and equalization of communication channels. More specifically, the thesis analyzed the performance of the considered adaptive processors when operating in the deficient sample support regime. In addition, it gained insights into behavior of different estimators based on the estimated second order statistics. Finally, it studied how to optimize the adaptive processors and algorithms so as to account for deficient sample support and consequently improve the performance.

\subsection{Random Matrix Theory}

The problems of adaptive processing in the deficient sample support have been analyzed and addressed by exploiting the results and insights from random matrix theory. The random matrix theory is a mathematical area which studies how eigenvalues and eigenvectors of a random matrix behave. It shows that certain encodings of the eigenvalues and eigenvectors of some random matrix ensembles exhibit deterministic behavior in the limit when the order of a matrix grows large. The estimate of the ensemble correlation matrix, the SCM, is a random matrix of our interest. From that hand, this thesis could be viewed as a study of what random matrix theory can teach as about adaptive processing in the deficient sample support regime.

More specifically, we have utilized the characterizations of the limiting behavior of eigenvalue and eigenvector Stieltjes transforms corresponding to different SCM models. The considered SCM models involve unity or non-unity forgetting factor and have zero or non-zero diagonal loading. Furthermore, the limiting moments 
corresponding to these SCM models have been evaluated using the Stieltjes transform method. Finally, the Gaussian tools characterizing the expected value and variance of a functional of Gaussian vector have been exploited in characterizing the behavior of the deviation of the quadratic forms involving the inverse of the SCM from their asymptotic counterparts.

In the following, we briefly summarize the problems considered in each application of adaptive processing and enumerate the contributions.

\subsection{Spatial Power Spectrum Estimation}

In the context of adaptive spatial power spectrum estimation, we have focused on two commonly used spatial power spectrum estimators based on the MPDR beamformer and studied how regularization in the form of diagonal loading, introduced with the goal to alleviate the problems caused by deficient sample support, impacts the estimation performance. The following results have been obtained.

First, it has been shown that both power estimators almost surely converge to deterministic limits when the number of sensors and observations (snapshots) grow large at the same rate. Given that both power estimators are bounded for finite value of diagonal loading, it has been concluded that the expectations of the power estimators converge to the same limits. Although asymptotic, as are the other results in large dimensional random matrix theory, due to rapid convergence, the deterministic limits fairly accurately approximate the expected values of power estimators for finite and relatively small number of sensors and snapshots.

Second, under the assumption that the snapshots are Gaussian distributed, the rate of convergence of one of the power estimators to its deterministic limit, its variance and estimation mean square error have been characterized. In doing so, the Gaussian tools which characterize the expectation and variance of a functional of a Gaussian vector have been exploited.

Third, a variety of results characterizing how the power estimators, their expec-

tations and variances depend on diagonal loading have been obtained. We have 
conjectured from these results that the variance has negligible impact on the value of optimal diagonal loading which minimizes the estimation mean square error, meaning that the optimal diagonal loading is controlled by the squared bias. The performance loss incurred by using the diagonal loading which minimizes the squared bias instead of the one which optimizes the estimation MSE has been shown to be insignificant.

Forth, using the stated conjecture, we have investigated how optimal diagonal loading depends on steering direction when the arrival model consists of plane waves contaminated with uncorrelated noise. We have shown that when steering close to the source direction, the optimal loading is small and can even be zero, meaning that the optimal estimator tends to perform adaptation as much as possible, fully relying on the available data. On the other hand, when steering direction moves away from the source direction, the optimal loading tends to increase and follows an oscillatory behavior.

Finally, the MSE performances of the two power estimators have been compared and it has been shown that the estimator $\hat{P}_{b}$ performs better (lower MSE) than the estimator $\hat{P}_{a}$ at the expense of increased sensitivity to optimal diagonal loading.

All obtained results are validated via Monte-Carlo simulations.

\subsection{Time-Varying Channel Tracking}

In the context of time-varying channel tracking problem, the performance of the Recursive Least Squares algorithm when used to estimate and track the channel whose variations are modeled with a first order Markov process has been studied and the following results have been obtained.

First, the channel estimation and signal prediction mean square errors are characterized in the deficient sample support regime.

Second, the general results are applied for specific scenarios and as special cases, the tracking performance in the steady-state, performance of the LS-based identification of linear time-invariant channel and performance of the sliding window RLS algorithm are characterized. 
Third, several practical results have been obtained using the developed analysis. As such, an expression for the optimal window length in the sliding window LS algorithm has been derived. Also, based on the comparison between the exponentially weighted and sliding window LS algorithms, it has been conjectured that former outperforms the latter, if forgetting factor is appropriately selected given the sliding window length. The corresponding expression for such a forgetting factor has been derived. Furthermore, a relation between forgetting factor used for calculating an exponentially weighted sample correlation matrix and the effective number of stationary, rectangularly windowed observations has been established. In addition, this relation has been further exploited to evaluate the optimal value of the forgetting factor in the exponentially weighted LS algorithm. Finally, the effect of performance deterioration appearing when the number of observations is close to channel length (i.e., the number of dimensions) is observed, theoretically analyzed and intuitively elaborated.

All obtained results are validated via Monte-Carlo simulations.

\subsection{Channel Equalization}

In the context of time-varying channel equalization, the problem of optimal configuring a multi-channel Decision Feedback Equalizer whose weights are evaluated and adapted using the least squares algorithm in the deficient sample support regime has been studied and the following results have been obtained.

First, the performance of the least squares based multi-channel DFE is theoretically characterized and it has been shown that the prediction MSE at the output of the LS-based MC-DFE equalizer is proportional to the signal prediction MSE at the output of the corresponding MMSE equalizer and the proportionality constant does not depend on the channel impulse response. The derived expression is validated via Monte-Carlo simulations.

Second, using the obtained characterization of the equalization performance it

has been highlighted that the optimal number of equalizer coefficients is a trade off 
between the MMSE requirement for longer filters and the insight that finite number of observations can effectively adapt only a limited number of equalizer weights. This is validated via Monte-Carlo simulations and processing of experimentally collected data.

Finally, it has been shown that as opposed to standard MIMO systems and conventional wisdom in array processing, the optimal separation between the sensors which minimize the signal prediction MSE is a more subtle problem. To study it, we have introduced an arrival model which accounts for wideband and spatially spread nature of the received underwater acoustic communication signals. Using a particular arrival structure we have shown that the performance is optimized for a specific selection of the number of sensors and their separation. Finally, these findings have been confirmed by the processing of experimental data.

\subsection{Future Work}

In terms of future work, some of the results on optimal diagonal loading for spatial power spectrum estimation could be strengthen and extended in several possible ways. In a shorter term, the stated conjecture about the negligible impact of variance on the value of optimal diagonal loading needs to be rigorously proved. In addition, a rigorous sensitivity analysis of power estimators on optimal diagonal loading is needed to complete the comparison of power estimators. Finally, the results developed for Gaussian distributed snapshots could possibly be extended to more general snapshot statistics.

In a longer term, the ultimate goal concerning the problem of diagonal loading for adaptive beamforming is to develop a scheme which determines, in real-time, the optimal diagonal loading to be used in the computation of the beamformer's weights based on the received data and steering direction. Furthermore, the presented study on how spatial power spectrum estimation depends on diagonal loading could be applied to other estimation methods which rely on diagonal loading and possibly generalized to other regularization approaches. Finally, the analysis of other regularization meth- 
ods applied to adaptive beamforming and spatial power spectrum estimation could possible benefit from the tools and approaches used in the thesis.

In the context of optimal equalizer design, the presented arrival model could be extended such that the dependence of optimal number of sensors on total wavenumber spread and wavenumber spread of the arrivals we aim to separate, is revealed. Also, the arrival model could be generalized to mimic more realistic arrival structures so as to include more arrivals, non-flat frequency response over the patches in the frequencywavenumber domain and to account for non-negligible delay within the arrival. A possible application of the developed results and insights might be in the design of subarray-based equalization method for rapidly varying communication channels.

The derived results and obtained insights in the problem of time-varying channel equalization could be possibly used for addressing problems arising in other applications. Namely, the derived characterization of the signal prediction MSE holds in other applications which use least squares based adaptation and consequently could be applied for studying issues caused by deficient sample support. In addition, the model developed for studying how the number of and separation between sensors impact the equalization performance could be adjusted to model sparse and spatially spread arrivals inherent to signals received in an ultra-wideband terrestrial communication system. An example is a communication system in an increasingly popular 60 $\mathrm{GHz}$ frequency band and possible problems that might be tackled using the developed arrival model are equalizer design and antenna selection in a massive MIMO receiver. 


\section{Bibliography}

[1] T. Adali and S. H. Ardalan. Fixed-point roundoff error analysis of the RLS algorithm with time-varying channels. International Conference on Acoustics, Speech, and Signal Processing, ICASSP, 3:1865-1868, 1991.

[2] M. Ainslie. Principles of Sonar Performance Modelling. Springer, 2010.

[3] A. B. Baggeroer and H. Cox. Passive sonar limits upon nulling multiple moving ships with large aperture arrays. 33rd Asilomar Conference on Signals, Systems, and Computers, pages 103-108, October 1999.

[4] Z. D. Bai and J. W. Silverstein. No eigenvalues outside of the support of the limiting spectral distribution of large dimensional sample covariance matrices. The Annals of Probability, 26(1):316-345, January 1998.

[5] B. C. Banister and J. R. Zeidler. Tracking performance of the RLS algorithm applied to an antenna array in a realistic fading environment. IEEE Transactions on Signal Processing, 50(5):1037-1050, 2002.

[6] F. Benaycg-Georges and N. Raj Rao. The singular values and vectors of low rank perturbations of large rectangular random matrices. Advances in Mathematics, 227(1), 2011.

[7] P. Billingsley. Probability and Measure. John Wiley and Sons, Inc., 1995.

[8] C. M. Bishop. Pattern Recognition and Machine Learning. Springer, 2006.

[9] J. P. Bouchaud and M. Potters. Financial applications of random matrix theory: A short review. Handbook on Random Matrix Theory, 2009. arXiv:0910.1205v1. 
[10] A. M. Bradley, M. D. Feezor, H. Singh, and F. Y. Sorrell. Power systems for autonomous underwater vehicles. IEEE Journal Of Oceanic Engineering, 26(4):526-538, October 2001.

[11] J. Capon and N. R. Goodman. Probability distributions for estimator of the frequency wavenumber spectrum. Proceedings of the IEEE, 58:1785-1786, 1970.

[12] R. F. W. Coates. Underwater Acoustic Systems. New York: Wiley, 1st edition, 1990.

[13] R. Couillet and M. Debbah. Random Matrix Methods for Wireless Communications. Cambridge University Press, 2011.

[14] R. Couillet, M. Debbah, and J. W. Silverstein. A deterministic equivalent for the analysis of correlated MIMO multiple access channels. IEEE Transactions on Information Theory, 57(6):3493-3514, 2011.

[15] A. Edelman. Eigenvalues of random matrices. Notes from the MIT's Class 18.338, Spring 2011.

[16] A. Edelman and N. Raj Rao. Random matrix theory. Acta Numerica, pages $1-65,2005$.

[17] E. Eleftheriou and D. D. Falconer. Tracking properties and steady-state performance of RLS adaptive filter algorithms. IEEE Transactions on Acoustics, Speech, and Signal Processing, 34(5):1097-1110, 1986.

[18] R. Everson and S. Roberts. Inferring the eigenvalues of covariance matrices from limited, noisy data. IEEE Transaction on Signal Processing, 48(7), 2000.

[19] E. Eweda. Comparison of RLS, LMS, and sign algorithms for tracking randomly time-varying channels. IEEE Transactions on Signal Processing, 42(11):29372944, 1994. 
[20] D. Gesbert, M. Shafi, D. Shiu, P. J. Smith, and A. Naguib. From theory to practice: An overview of MIMO space-time coded wireless systems. IEEE Journal on Selected Areas in Communications, 21(3):281-302, 2003.

[21] V. Girko. Strong law for the eigenvalues and eigenvectors of empirical covariance matrices. Random Oper. Stoch. Eq., 4:176-204, 1996.

[22] V. L. Girko. An Introduction to Statistical Analysis of Random Arrays. The Netherlands: VSP, 1998.

[23] Y. Gong and C. F. N. Cowan. Optimum decision delay of the finite-length DFE. IEEE Signal Processing Letters, 11(11):858-861, 2004.

[24] Y. Gong and C. F. N. Cowan. A self structured adaptive decision feedback equalizer. IEEE Signal Processing Letters, 13(3):169-172, 2006.

[25] W. Hachem, O. Khorunzhiy, P. Loubaton, J. Najim, and L. Pastur. A new approach for mutual information analysis of large dimensional multi-antenna channels. IEEE Transaction on Information Theory, 54(9), 2008.

[26] M. Hayes. Statistical Digital Signal Processing and Modeling. John Wiley and Sons, 1996.

[27] S. Haykin. Adaptive Filter Theory. Prentice Hall, 4th edition, 2002.

[28] W. Hodgkiss and J. Preisig. Kauai acomms muri 2011 (KAM11) experiment. Proc. 11th European Conf. Underwater Acoustics, 2012.

[29] S. M. Key. Fundamentals of Statistical Signal Processing, Volume I: Estimation Theory. Prentice Hall, 1993.

[30] S. Kritchman and B. Nadler. Non-parametric detection of the number of signals: Hypothesis testing and random matrix theory. IEEE Transactions on Signal Processing, 57(10):3930-3941, 2009. 
[31] E. Larsson, O. Edfors, F. Tufvesson, and T. Marzetta. Massive MIMO for next generation wireless systems. IEEE Communications Magazine, 52(2):186-195, February 2014.

[32] W. S. Leon and D. P. Taylor. Steady-state tracking analysis of the RLS algorithm for time-varying channels: a general state-space approach. IEEE Communications Letters, 7(5):236-238, 2003.

[33] V. A. Marcenko and L. A. Pastur. Distribution of eigenvalues in certain sets of random matrices. Mat. Sb. (N.S.), 72(114):507-536, 1967.

[34] J. M. Mendel. Tutorial on higher-order statistics (spectra) in signal processing and system theory: Theoretical results and some applications. Proceedings of the IEEE, 79(3):278-305, March 1991.

[35] X. Mestre and M. A. Lagunas. Finite sample size effect on minimum variance beamformers: Optimum diagonal loading factor for large arrays. IEEE Transaction on Signal Processing, 54(1), 2006.

[36] G. V. Moustakides. Study of the transient phase of the forgetting factor RLS. IEEE Transactions on Signal Processing, 45(10):2468-2476, 1997.

[37] Raj Rao Nadakuditi. Applied Stochastic Eigenanalysis. PhD thesis, MIT, 2007.

[38] M. J. M. Peacock, I. B. Collings, and M. L. Honig. Unified large-system analysis of MMSE and adaptive least squares receivers for a class of random matrix channels. IEEE Transactions on Information Theory, 52(8):3567-3600, 2006.

[39] J. C. Preisig. Performance analysis of adaptive equalization for coherent acoustic communications in the time-varying ocean environment. Journal of Acoustical Society of America, pages 263-278, July 2005.

[40] J. C. Preisig. Subarray partitioning for adaptive multichannel equalization. Proc. 11th European Conference on Underwater Acoustics (ECUA 2012), pages 16981702, July 2012. 
[41] J. Proakis. Digital Communications. McGraw-Hill Education, 4th edition, 2001.

[42] N. Raj Rao and A. Edelman. Sample eigenvalue based detection of highdimensional signals in white noise using relatively few samples. IEEE Transactions on Signal Processing, 56(7):2625-2638, 2008.

[43] I. S. Reed, J. D. Mallett, and L. E. Brennan. Rapid convergence rate in adaptive arrays. IEEE Transactions on Aerospace and Electronic Systems, AES10(6):853-863, Nov 1974.

[44] F. Rubin, X. Mestre, and W. Hachem. A CLT on the SINR of the diagonally loaded Capon/MVDR beamformer. ICASSP, 2011.

[45] F. Rubin, X. Mestre, and W. Hachem. A CLT on the SNR of diagonally loaded MVDR filters. IEEE Transactions on Signal Processing, 60(8):4178-4195, 2012.

[46] B. Schmandt and R. Clayton. Analysis of P-waves with a 5200-station array in Long Beach, California: Evidence for abrupt boundary for Inner Borderland rifting. J. Geophys. Res., 118:1-19, 2013.

[47] X. Shi, S. Leung, and C. Leung. Mean square error analysis of RLS algorithm for WSSUS fading channels. IEEE International Symposium on Circuits and Systems, ISCAS, pages 1-4, 2006.

[48] A. C. Singer, J. K. Nelson, and S. S. Kozat. Signal processing for underwater acoustic communications. IEEE Communications Magazine, 47(1):90-96, 2009.

[49] B. Sklar. Rayleigh fading channels in mobile digital communication systems Part I: Characterization. IEEE Communications Magazine, 35(7):90-100, 1997.

[50] B. Sklar. Rayleigh fading channels in mobile digital communication systems Part II: Mitigation. IEEE Communications Magazine, 35(7):102-109, 1997.

[51] M. Stojanovic and J. C. Preisig. Underwater acoustic communication channels: Propagation models and statistical characterization. IEEE Communications Magazine, 47(1):84-89, 2009. 
[52] H. L. Van Trees. Optimum Array Processing: Detection, Estimation and Modulation Theory. John Wiley and Sons, 2002.

[53] D. N. C. Tse and S. V. Hanly. Linear multiuser receivers: Effective interference, effective bandwidth and user capacity. IEEE Transactions on Information Theory, 45(2):641-657, February 1999.

[54] A. M. Tulino and S. Verdu. Random Matrix Theory and Wireless Communications. Now Publishers Inc, 2004.

[55] A. Vakili, E. Familier, and B. Hassibi. On the eigendistribution of the steadystate error covariance matrix for the extended RLS algorithm. IEEE International Conference on Acoustics, Speech and Signal Processing ICASSP, pages 2829-2832, 2009.

[56] A. Vakili and B. Hassibi. A Stieltjes transform approach for analyzing the RLS adaptive filter. 46th Annual Allerton Conference on Communication, Control, and Computing, pages 432-437, 2008.

[57] J.-J. van de Beek, O. Edfors, M. Sandell, S. K. Wilson, and P. O. Borjesson. On channel estimation in OFDM systems. IEEE 45th Vehicular Technology Conference, 2:815-819, 1995.

[58] P. A. van Walree, T. Jenserud, and M. Smedsrud. A discrete-time channel simulator driven by measured scattering functions. IEEE Journal on Selected Areas in Communications, 26(9):1628-1637, 2008.

[59] E. Wigner. Characteristic vectors of borded matrices with infinite dimension. The Annales of Mathematics, 62:548-564, November 1955.

[60] E. Wigner. On the distribution of roots of certain symmetric matrices. The Annales of Mathematics, 67:325-328, March 1958.

[61] J. Wishart. The generalised product moment distribution in samples from a normal multivariate population. Biometrica, 20A(1-2):32-52, 1928. 
[62] W. Xiao and M. L. Honig. Large system transient analysis of adaptive least squares filtering. IEEE Transactions on Information Theory, 51(7):2447-2474, 2005.

[63] B. Xie, T. Bose, and Z. Zhang. Performance analysis of deficient-length RLS and EDS algorithms. IEEE 13th Digital Signal Processing Workshop and 5th IEEE Signal Processing Education Workshop, pages 115-120, 2009.

[64] L. Yang and G. B. Giannakis. Ultra-wideband communications: An idea whose time has come. IEEE Signal Processing Magazine, 21(6):26-54, Nov 2004.

[65] L. Zhang. Spectral analysis of large dimensional random matrices. PhD thesis, National University of Singapore, 2006. 


\section{Appendix A}

\section{Proof of Lemma 3.5}

A quadratic form $Q_{k}$ can be written in terms of the eigen-decomposition of the SCM $\hat{\mathbf{R}}$ as

$$
Q_{k}(\delta)=\sum_{i=1}^{m} \frac{\left|\mathbf{v}_{s}^{H} \hat{\mathbf{q}}_{i}\right|^{2}}{\left(\hat{\lambda}_{i}+\delta\right)^{k}} .
$$

Due to assumptions 2 and $3, \hat{d}_{m} \leq \hat{\lambda}_{i} \leq \hat{D}_{m}$ and thus

$$
\frac{m}{\left(\hat{D}_{m}+\delta\right)^{k}} \leq Q_{k}(\delta) \leq \frac{m}{\left(\hat{d}_{m}+\delta\right)^{k}}
$$

The derivatives of the estimators $\hat{P}_{a}$ and $\hat{P}_{b}$ are expressed in terms of quantities $Q_{k}$ 's as

$$
\begin{aligned}
\frac{\partial \hat{P}_{a}}{\partial \delta} & =\frac{2 \delta}{Q_{1}}\left(\frac{Q_{3}}{Q_{1}}-\frac{Q_{2}^{2}}{Q_{1}^{2}}\right) . \\
\frac{\partial \hat{P}_{b}}{\partial \delta} & =\frac{Q_{2}}{Q_{1}^{2}} .
\end{aligned}
$$

Combining (A.1) with (A.2) and (A.3) yields

$$
\frac{\partial \hat{P}_{a}}{\partial \delta} \leq \frac{2 \delta\left(\hat{D}_{m}+\delta\right)^{3}}{m}\left[\frac{1}{\left(\hat{d}_{m}+\delta\right)^{4}}-\frac{1}{\left(\hat{D}_{m}+\delta\right)^{4}}\right]
$$


and

$$
\frac{1}{m}\left(\frac{\hat{d}_{m}+\delta}{\hat{D}_{m}+\delta}\right)^{2} \leq \frac{\partial \hat{P}_{b}}{\partial \delta} \leq \frac{1}{m}\left(\frac{\hat{D}_{m}+\delta}{\hat{d}_{m}+\delta}\right)^{2}
$$

which completes the proof. 


\section{Appendix B}

\section{Proof of Lemma 3.6}

The claims are proved by using the Poincare-Nesh inequality (2.67) wherein $f(\mathbf{Y})$ is one of the power estimators or their derivatives. Since the received snapshots are Gaussian distributed, we stick to the equivalent representation of the quadratic forms given in (3.21).

In proving Lemma 3.6, the following facts are used

- The norm of the resolvent matrix is almost surely upper bounded by 1 , i.e.,

$$
\|\mathbf{H}\| \leq 1
$$

To confirm, note that its all eigenvalues are between zero and one.

- The derivative of $H_{p g}$ with respect to $Y_{i j}^{*}$ is given by [25]

$$
\frac{\partial H_{p q}}{\partial Y_{i j}^{*}}=-\frac{t}{n}(H Y)_{p j} H_{i q} .
$$

- For $k \geq l$,

$$
\frac{Q_{k}}{Q_{l}} \leq t^{k-l}
$$

This can be checked by writing $Q_{k}$ and $Q_{l}$ in terms of their eigendecompositions and noting that $\left(1+t \hat{\lambda}_{i}\right)^{k} \geq\left(1+t \hat{\lambda}_{i}\right)^{l}$ because $1+t \hat{\lambda}_{i} \geq 1$. 
As a preliminary step, we upper bound a quantity $T_{k}$, defined by

$$
T_{k}=\sum_{i, j} \lambda_{i} \mathbf{E}\left[\left|\frac{\partial Q_{k}}{\partial Y_{i j}^{*}}\right|^{2}\right] .
$$

In doing so, we first need to evaluate the corresponding derivatives of $Q_{k}$ 's. For $k=2$, the derivative of $Q_{2}$ is computed via

$$
\begin{aligned}
& \frac{\partial Q_{2}}{\partial Y_{i j}^{*}}=t^{2} \sum_{p, q, u} s_{p}^{*} \frac{\partial\left(H_{p q} H_{q u}\right)}{\partial Y_{i j}^{*}} s_{u} \\
& \stackrel{(a)}{=}-\frac{t^{3}}{n} \sum_{p, q, u} s_{p}^{*}\left[(H Y)_{p j} H_{i q} H_{q u}+H_{p q}(H Y)_{q j} H_{i u}\right] s_{u} \\
& =-\frac{t^{3}}{n} \sum_{l=1}^{2}\left(\mathbf{s}^{H} \mathbf{H}^{l} \mathbf{Y}\right)_{j}\left(\mathbf{H}^{3-l} \mathbf{s}\right)_{i},
\end{aligned}
$$

where $\stackrel{(a)}{=}$ follows from (B.2). Given (B.5), it is easily generalized that

$$
\frac{\partial Q_{k}}{\partial Y_{i j}^{*}}=\sum_{l=1}^{k}\left(\mathbf{s}^{H} \mathbf{H}^{l} \mathbf{Y}\right)_{j}\left(\mathbf{H}^{k+1-l} \mathbf{s}\right)_{i} .
$$

Now, substituting (B.6) into (B.4), the upper bound for $T_{k}$ is found as follows

$$
\begin{aligned}
& T_{k}=\frac{t^{2(k+1)}}{n^{2}} \sum_{i, j} \lambda_{i} \mathbf{E}\left[\left|\sum_{l=1}^{k}\left(\mathbf{s}^{H} \mathbf{H}^{l} \mathbf{Y}\right)_{j}\left(\mathbf{H}^{k+1-l} \mathbf{s}\right)_{i}\right|^{2}\right] \\
& \stackrel{(a)}{\leq} \frac{k t^{2(k+1)}}{n^{2}} \sum_{l=1}^{k} \sum_{i, j} \lambda_{i} \mathbf{E}\left[\left|\left(\mathbf{s}^{H} \mathbf{H}^{l} \mathbf{Y}\right)_{j}\left(\mathbf{H}^{k+1-l} \mathbf{s}\right)_{i}\right|^{2}\right] \\
& =\frac{k t^{2(k+1)}}{n^{2}} \sum_{l=1}^{k} \mathbf{E}\left[\left(\mathbf{s}^{H} \mathbf{H}^{l} \mathbf{Y} \mathbf{Y}^{H} \mathbf{H}^{l} \mathbf{s}\right)\left(\mathbf{s}^{H} \mathbf{H}^{k+1-l} \mathbf{D} \mathbf{H}^{k+1-l} \mathbf{s}\right)\right],
\end{aligned}
$$

where $\stackrel{(a)}{\leq}$ follows from the inequality

$$
\left(\sum_{i=1}^{k} a_{i}\right)^{2} \leq k\left(\sum_{i=1}^{k}\left|a_{i}\right|^{2}\right) .
$$


The summand in (B.10) is upper bounded as follows

$$
\begin{aligned}
& \mathbf{E}\left[\left(\mathbf{s}^{H} \mathbf{H}^{l} \mathbf{Y} \mathbf{Y}^{H} \mathbf{H}^{l} \mathbf{s}\right)\left(\mathbf{s}^{H} \mathbf{H}^{k+1-l} \mathbf{D} \mathbf{H}^{k+1-l} \mathbf{s}\right)\right] \\
& \stackrel{(a)}{\leq} \mathbf{E}\left[\|\mathbf{s}\|^{4}\|\mathbf{H}\|^{2(k+1)}\|\mathbf{D}\|\left\|\mathbf{Y} \mathbf{Y}^{H}\right\|\right] \\
& \stackrel{(b)}{\leq} \mathbf{E}\left[S_{m}^{4} H_{m}^{2(k+1)} D_{m} \sqrt{\left(\mathbf{Y} \mathbf{Y}^{H}\right)^{2}}\right] \\
& \stackrel{(c)}{\leq} S_{m}^{4} D_{m} n \sqrt{\mathbf{E}\left[\left(\frac{\mathbf{Y} \mathbf{Y}^{H}}{n}\right)^{2}\right]} \\
& =S_{m}^{4} D_{m} n \sqrt{\mathbf{E}\left[\sum_{i=1}^{m} \hat{\lambda}_{i}^{2}\right]} \\
& \stackrel{(d)}{\leq} S_{m}^{4} D_{m} \hat{D}_{m} n \sqrt{m}
\end{aligned}
$$

where $\stackrel{(a)}{\leq}$ follows from the Cauchy-Schwartz inequality for matrix norms, i.e., $\|\mathbf{A B}\| \leq$ $\|\mathbf{A}\|\|\mathbf{B}\|$. The inequality $\stackrel{(b)}{\leq}$ follows from the boundedness of the signal replica vector and of the matrices $\mathbf{H}$ and $\mathbf{D}$, i.e., $\|\mathbf{s}\| \leq S_{m},\|\mathbf{D}\| \leq D_{m}$ and $\|\mathbf{H}\| \leq 1$. The inequality $\stackrel{(c)}{\leq}$ follows from the Cauchy-Schwartz inequality for the expectation operator. Finally, $\stackrel{(d)}{\leq}$ follows from the boundedness of the eigenvalues of the SCM $\hat{\mathbf{R}}$.

The upper bound of $T_{k}$ is finally obtained by substituting (B.9) into (B.10) such that

$$
T_{k} \leq k^{2} t^{2(k+1)} S_{m}^{4} D_{m} \hat{D}_{m} \frac{\sqrt{m}}{n}
$$

Back to the variance problem, it can be shown that $f(\mathbf{Y})$ being $\hat{P}$ or $\frac{\partial \hat{P}}{\partial \delta}, \frac{\partial f(\mathbf{Y})}{\partial Y_{i j}^{*}}=$ $\frac{\partial f(\mathbf{Y})}{\partial Y_{i j}}$ such that

$$
\operatorname{var}(f(\mathbf{Y})) \leq 2 \sum_{i, j} \lambda_{i} \mathbf{E}\left[\left|\frac{\partial f(\mathbf{Y})}{\partial Y_{i j}^{*}}\right|^{2}\right]
$$

In the following, the variance of each of the estimators and their derivatives is upper bounded. The derivation for $f(\mathbf{Y})=\hat{P}_{a}$ is given in more details. Other derivations are similar and thus just outlined. 
Upper Bound of the Variance of $f(\mathbf{Y})=\hat{P}_{a}$

A derivative of $\hat{P}_{a}$ with respect to $Y_{i j}^{*}$ is in terms of $Q_{k}$ expressed as

$$
\frac{\partial \hat{P}_{a}}{\partial Y_{i j}^{*}}=\frac{1}{t Q_{1}^{2}}\left[\left(2 \frac{Q_{2}}{Q_{1}}-t\right) \frac{\partial Q_{1}}{\partial Y_{i j}^{*}}-\frac{\partial Q_{2}}{\partial Y_{i j}^{*}}\right] .
$$

Using (B.8), the squared magnitude of (B.12) is upper bounded as

$$
\left|\frac{\partial \hat{P}_{a}}{\partial Y_{i j}^{*}}\right|^{2} \leq \frac{2}{t^{2} Q_{1}^{4}}\left[\left(2 \frac{Q_{2}}{Q_{1}}-t\right)^{2}\left|\frac{\partial Q_{1}}{\partial Y_{i j}^{*}}\right|^{2}+\left|\frac{\partial Q_{2}}{\partial Y_{i j}^{*}}\right|^{2}\right]
$$

The coefficient of the first term in the above expression is using (B.8) and (B.3) upper bounded by $10 t^{2}$. Further, using (A.1) to lower bound the quadratic form $Q_{1}$ yields

$$
\left|\frac{\partial \hat{P}_{a}}{\partial Y_{i j}^{*}}\right|^{2} \leq \frac{2\left(1+t \hat{D}_{m}\right)^{4}}{t^{6} m^{4}}\left[10 t^{2}\left|\frac{\partial Q_{1}}{\partial Y_{i j}^{*}}\right|^{2}+\left|\frac{\partial Q_{2}}{\partial Y_{i j}^{*}}\right|^{2}\right] .
$$

Substituting (B.14) into (B.11) yields

$$
\begin{aligned}
\operatorname{var}\left(\hat{P}_{a}\right) & \leq \frac{2\left(1+t \hat{D}_{m}\right)^{4}}{t^{6} m^{4}}\left[10 t^{2} T_{1}+T_{2}\right] \\
& \stackrel{(a)}{\leq} 28\left(1+t \hat{D}_{m}\right)^{4} D_{m} \hat{D}_{m} S_{m}^{4} \frac{\sqrt{m}}{n m^{4}} \\
& \stackrel{(b)}{\leq} \frac{K}{m^{5 / 2}},
\end{aligned}
$$

where $\stackrel{(a)}{\leq}$ follows from (B.10) and $\stackrel{(a)}{\leq}$ from the assumption that $m$ and $n$ are of the same order and $S_{m}=O\left(m^{1 / 2}\right)$.

\section{Upper Bound of the Variance of $f(\mathbf{Y})=\hat{P}_{b}$}

Similarly as in the previous part, the derivative of $\hat{P}_{b}$ with respect to $Y_{i j}^{*}$ is given by

$$
\frac{\partial \hat{P}_{b}}{\partial Y_{i j}^{*}}=\frac{1}{t Q_{1}^{2}} \frac{\partial Q_{1}}{\partial \delta}
$$


Substituting (B.16) into (B.11) yields

$$
\operatorname{var}\left(\hat{P}_{b}\right) \leq \frac{2}{t^{2} Q_{1}^{4}} T_{1}
$$

Exploiting the upper bound for $T_{1}$ (B.10) and the lower bound for $Q_{1}$ (A.1) leads to the upper bound for the variance of $\hat{P}_{b}$

$$
\operatorname{var}\left(\hat{P}_{b}\right) \leq 2 t^{-2}\left(1+t \hat{D}_{m}\right)^{4} D_{m} \hat{D}_{m} S_{m} \frac{\sqrt{m}}{n m^{4}}
$$

Thus, under the assumption that $m$ and $n$ are of the same order and $S_{m}=O(\sqrt{m})$, $\operatorname{var}\left(\hat{P}_{b}\right) \leq K m^{-\frac{5}{2}}$, for some positive finite constant $K$.

\section{Upper Bound of the Variance of $f(\mathbf{Y})=\frac{\partial \hat{P}_{a}}{\partial \delta}$}

A derivative of $\hat{P}_{a}^{\prime}=\frac{\partial \hat{P}_{a}}{\partial \delta}$ with respect to $Y_{i j}^{*}$ is in terms of $Q_{k}$ 's expressed as

$$
\frac{\partial \hat{P}_{a}^{\prime}}{\partial Y_{i j}^{*}}=\frac{2}{t Q_{1}^{2}}\left[\left(3 \frac{Q_{2}^{2}}{Q_{1}^{2}}-2 \frac{Q_{3}}{Q_{1}}\right) \frac{\partial Q_{1}}{\partial Y_{i j}^{*}}-2 \frac{Q_{2}}{Q_{1}} \frac{\partial Q_{2}}{\partial Y_{i j}^{*}}+\frac{\partial Q_{3}}{\partial Y_{i j}^{*}}\right]
$$

The squared magnitude of the above expression is using (B.8) and (B.3) upper bounded as

$$
\left|\frac{\partial \hat{P}_{a}^{\prime}}{\partial Y_{i j}^{*}}\right| \leq \frac{12}{t^{2} Q_{1}^{4}}\left(26 t^{4}\left|\frac{\partial Q_{1}}{\partial Y_{i j}^{*}}\right|^{2}+4 t^{2}\left|\frac{\partial Q_{2}}{\partial Y_{i j}^{*}}\right|^{2}+\left|\frac{\partial Q_{3}}{\partial Y_{i j}^{*}}\right|^{2}\right)
$$

Substituting (B.20) into (B.11) and using the upper bounds for $T_{k}$ (B.10) and lower bound for $Q_{1}$ (A.1), the variance of $\hat{P}_{a}^{\prime}$ is upper bounded as

$$
\operatorname{var}\left(\hat{P}_{b}\right) \leq 612 t^{2}\left(1+t \hat{D}_{m}\right)^{4} D_{m} \hat{D}_{m} S_{m}^{4} \frac{\sqrt{m}}{n m^{4}}
$$

i.e., if $S_{m}=O(\sqrt{m})$ and $m$ and $n$ are of the same order, $\operatorname{var}\left(\hat{P}_{b}\right) \leq K m^{-\frac{5}{2}}$, where $K$ is some positive, finite constant. 
Upper Bound of the Variance of $f(\mathbf{Y})=\frac{\partial \hat{P}_{b}}{\partial \delta}$

A derivative of $\hat{P}_{b}^{\prime}=\frac{\partial \hat{P}_{b}}{\partial \delta}$ with respect to $Y_{i j}^{*}$ is in terms of $Q_{k}$ 's expressed as

$$
\frac{\partial \hat{P}_{b}^{\prime}}{\partial Y_{i j}^{*}}=\frac{1}{Q_{1}^{2}}\left[\frac{\partial Q_{2}}{\partial Y_{i j}^{*}}-2 \frac{Q_{2}}{Q_{1}} \frac{\partial Q_{1}}{\partial Y_{i j}^{*}}\right]
$$

The squared magnitude of the above expression is using (B.8) and (B.3) upper bounded as

$$
\left|\frac{\partial \hat{P}_{b}^{\prime}}{\partial Y_{i j}^{*}}\right|^{2} \leq \frac{1}{Q_{1}^{4}}\left(\left|\frac{\partial Q_{2}}{\partial Y_{i j}^{*}}\right|^{2}+4 t^{2}\left|\frac{\partial Q_{1}}{\partial Y_{i j}^{*}}\right|^{2}\right) .
$$

Substituting (B.20) into (B.11) and using the upper bounds for $T_{k}$ (B.10) and lower bound for $Q_{1}$ (A.1) lead to

$$
\operatorname{var}\left(\hat{P}_{b}\right) \leq 20 t^{2}\left(1+t \hat{D}_{m}\right)^{4} D_{m} \hat{D}_{m} S_{m}^{4} \frac{\sqrt{m}}{n m^{4}}
$$

Therefore, under the assumptions that $m$ and $n$ are of the same order and $S_{m}=$ $O(\sqrt{m}), \operatorname{var}\left(\hat{P}_{b}\right) \leq K m^{-\frac{5}{2}}$, where $K$ is some positive, finite constant. 


\section{Appendix C}

\section{Optimization Loss}

Let $f(x)=a(x-\tilde{x})^{2}$ and $g(x)=b(x-\tilde{x})+c$ be respectively quadratic and linear functions. The function $f(x)$ reaches minimum at $x=\tilde{x}$. On the other hand, the minimizer for the sum $h(x)=f(x)+g(x)$ is after setting the first derivative of $h(x)$ to zero given by

$$
x^{*}=\tilde{x}-\frac{b}{2 a} .
$$

Therefore, the minimum of $h(x)$ is

$$
h\left(x^{*}\right)=-\frac{b^{2}}{4 a}+c
$$

The error made by setting the minimizer of $h(x)$ to be $\tilde{x}$ instead of $x^{*}$ is

$$
h(\tilde{x})-h\left(x^{*}\right)=\frac{b^{2}}{4 a} .
$$

This result is used to approximate the MSE loss made by optimizing the squared bias instead of the sum of the squared bias and variance. Therein, the approximation of the squared bias is $f(x)$, while the approximation of the variance is $g(x)$. 


\section{Nomenclature}

$m$ number of coefficients (dimensionality of the observation space)

$\mathbf{q}_{k} \quad$ the eigenvector of the ensemble correlation matrix corresponding to $\lambda_{k}$

$\hat{\lambda}_{k} \quad$ the $k-t h$ largest eigenvalue of the sample correlation matrix

$\hat{\mathbf{q}}_{k} \quad$ the eigenvector of the sample correlation matrix corresponding to $\hat{\lambda}_{k}$

r cross-correlation vector

$\hat{\mathbf{r}} \quad$ estimate of the cross-correlation vector

$G^{\mathbf{A}_{m}}(x)$ empirical Eigenvalue Distribution Function corresponding to $\mathbf{A}_{m}$ $\mu_{\mathbf{A}_{m}}(x)$ empirical Eigenvalue Density Function corresponding to $\mathbf{A}_{m}$ $\mu_{\mathbf{A}}(x)$ limiting Eigenvalue Density Function

$S_{\mathbf{A}_{m}}(z)$ empirical Eigenvalue Stieltjes Transform corresponding to $\mathbf{A}_{m}$

$\bar{S}_{\mathbf{A}}(z)$ limiting Eigenvalue Stieltjes Transform

$F_{\mathbf{A}_{m}}(z)$ empirical Eigenvector Stieltjes Transform corresponding to $\mathbf{A}_{m}$

$n \quad$ number of observations (snapshots, samples)

$\bar{F}_{\mathbf{A}}(z)$ limiting Eigenvector Stieltjes Transform

$M_{k} \quad$ empirical $k$-th moment of a random matrix

$\bar{M}_{k} \quad$ limiting $k$-th moment of a random matrix 
$\tilde{M}_{k} \quad$ expectation of the $k$-th empirical moment of a random matrix

$\tilde{M}_{k}^{\infty}$ expectation of the $k$-th empirical moment of a matrix in the limit when its order grows large

$\lambda \quad$ forgetting factor

$\lambda_{\text {opt }}$ optimal forgetting factor

w vector adaptive processor weights

$d \quad$ separation between sensors in a line array

$\mathbf{v}_{s} \quad$ signal replica vector

$N \quad$ number of sensors

$\theta \quad$ elevation angle

$\hat{P} \quad$ power estimate

$P \quad$ true power

$\operatorname{MSE}(\delta)$ estimation MSE of power estimator for diagonal loading $\delta$

$\operatorname{bias}^{2}(\delta)$ squared bias of power estimator for diagonal loafing $\delta$

$\operatorname{var}(\delta)$ variance of power estimator for diagonal loafing $\delta$

$\delta \quad$ diagonal loading

$\delta_{\text {opt }} \quad$ diagonal loading which minimizes $\operatorname{MSE}(\delta)$

$\tilde{\delta}_{\text {opt }} \quad$ diagonal loading which minimizes $\operatorname{bias}^{2}(\delta)$

$v(n)$ observation noise at discrete time $n$

c ratio between the number of coefficients and number of observations

$d(n)$ channel output at time $n$ 
$\sigma_{v}^{2} \quad$ variance of the observation noise

$\sigma_{o}^{2} \quad$ variance of the process noise

$\boldsymbol{\epsilon} \quad$ channel estimation error

$\xi \quad$ signal prediction error

$L_{\mathrm{ff}} \quad$ feedforward filter length

$L_{\mathrm{fb}} \quad$ feedback filter length

$x_{\text {soft }} \quad$ soft decision estimate

$\sigma_{\text {MMSE }}^{2}$ signal prediction MSE of the MMSE processor

$\sigma_{\mathrm{LS}}^{2} \quad$ signal prediction MSE of the LS processor

$\mathbf{u}(n)$ observation vector received at discrete time $n$

R ensemble correlation matrix

$\hat{\mathbf{R}} \quad$ sample correlation matrix

$\hat{\mathbf{R}}_{\delta} \quad$ diagonally loaded sample correlation matrix

$\lambda_{k} \quad$ the $k$-th largest eigenvalue of the ensemble correlation matrix 